\title{
West Foster Creek Expansion Project 2007 HEP Report
}

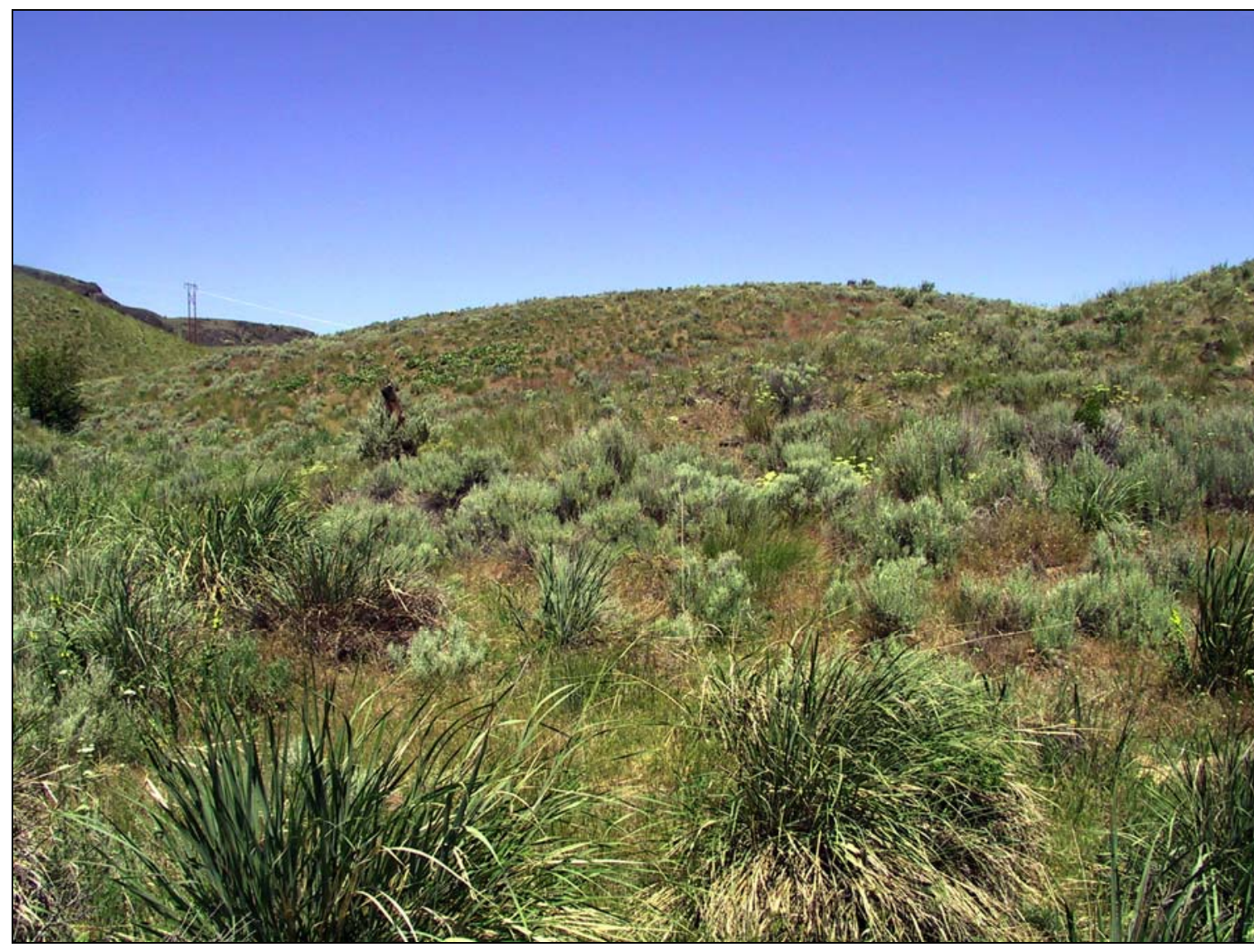

Prepared By

Paul R Ashley - RHT

For

Joe DeHerrera - BPA

And

Nate Pamplin - WDFW

February 2008 


\section{Table of Contents}

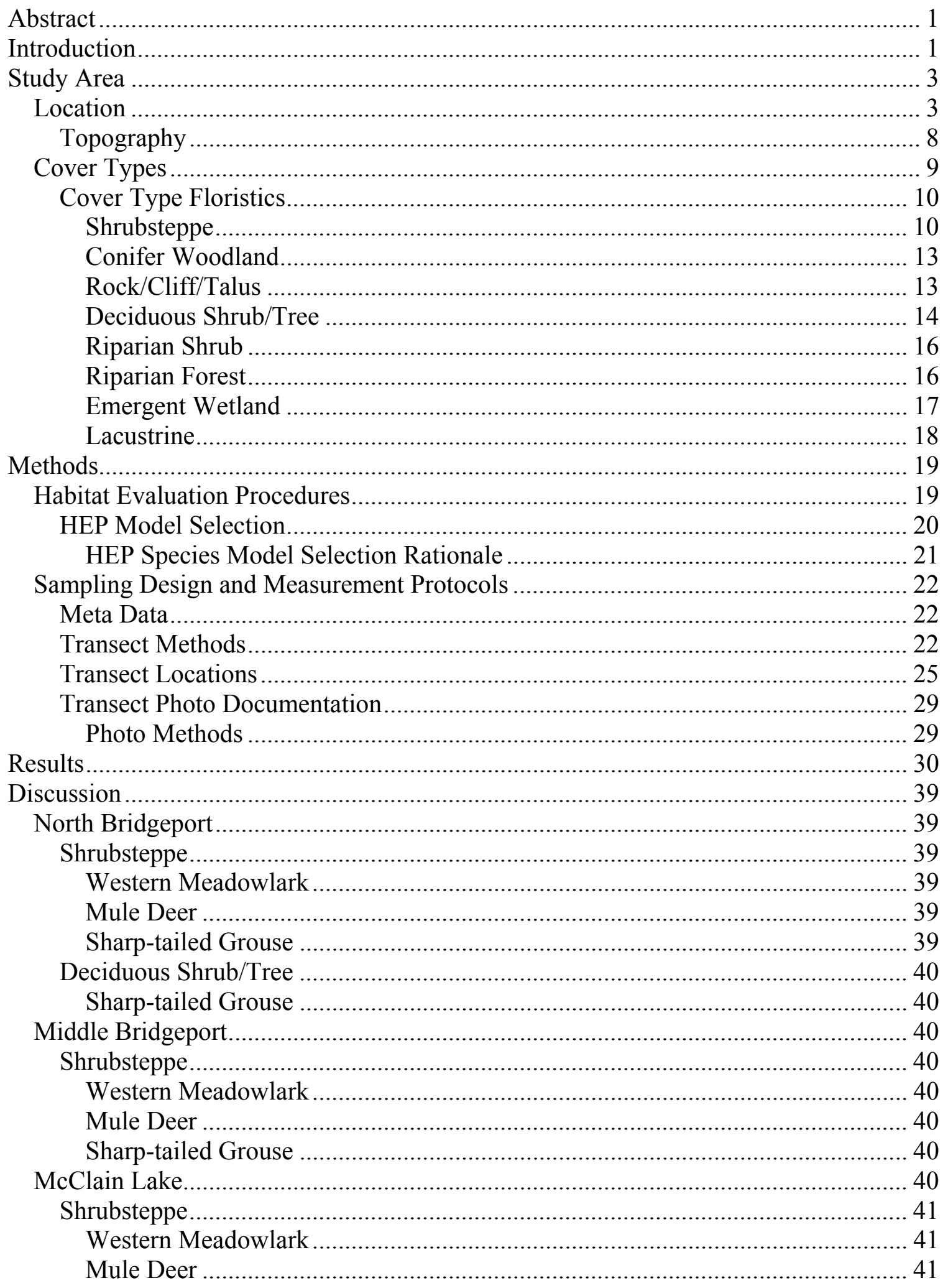




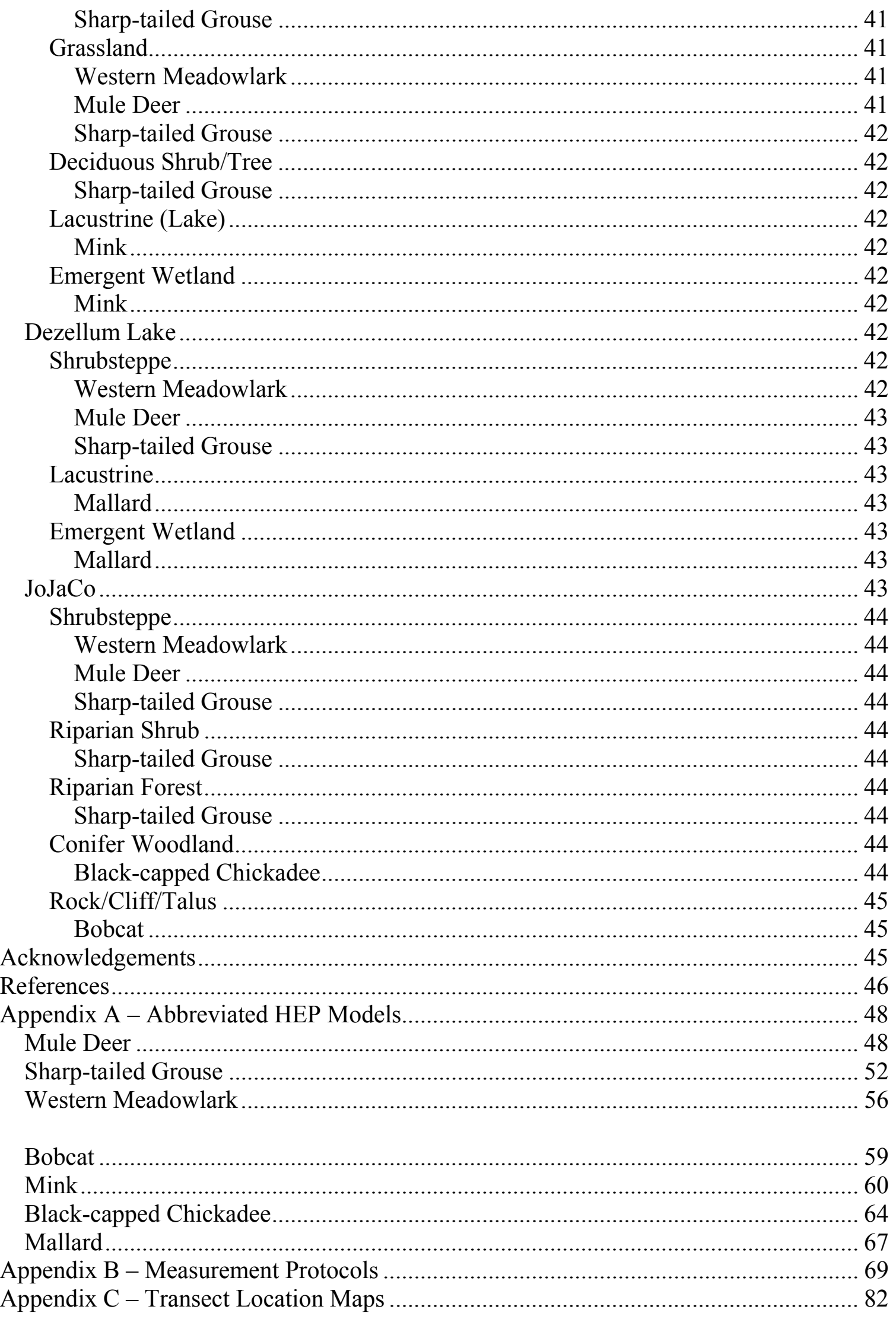




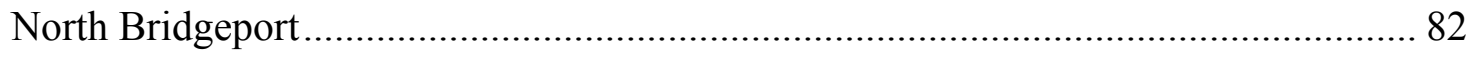

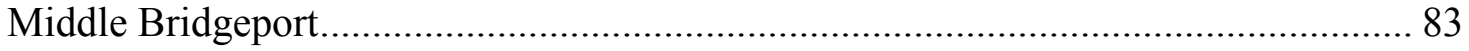

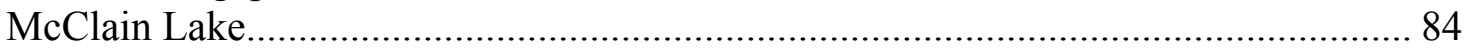

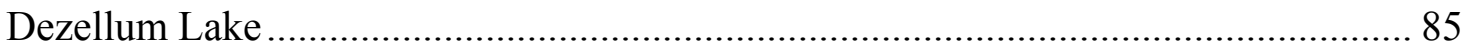

JoJaCo

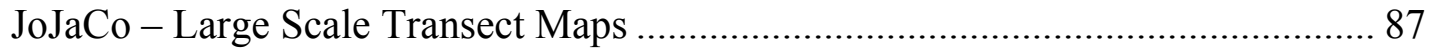

Appendix D - Transect Photographs .................................................................... 92

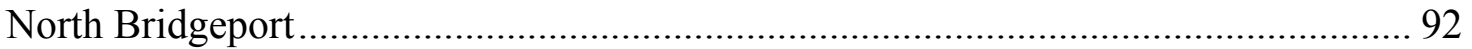

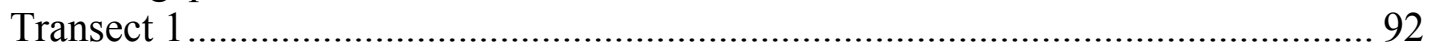

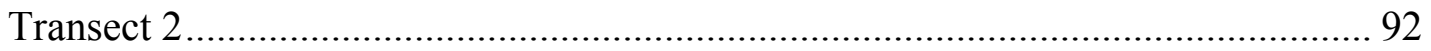

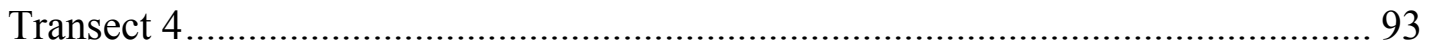

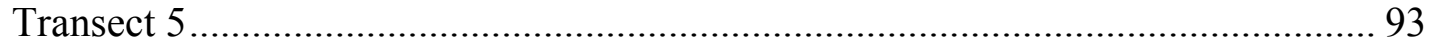

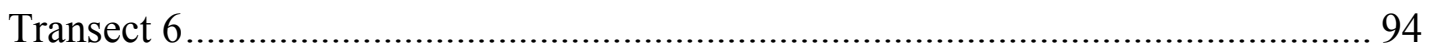

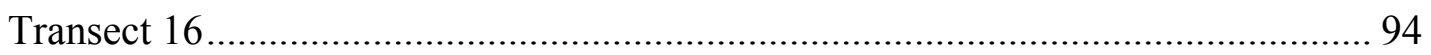

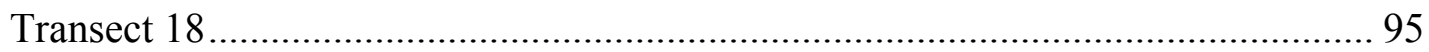

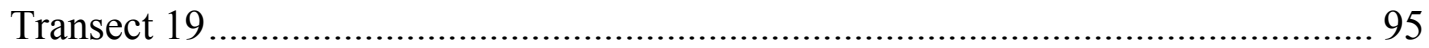

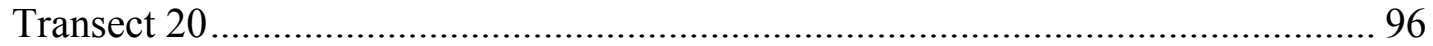

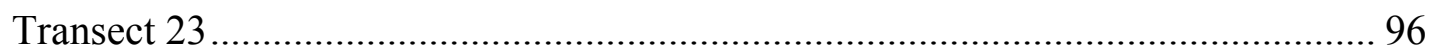

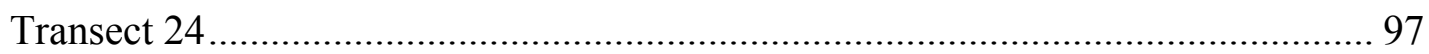

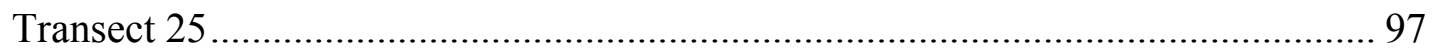

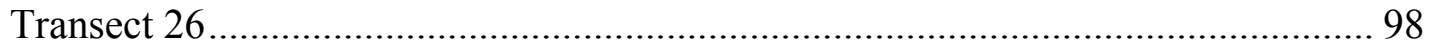

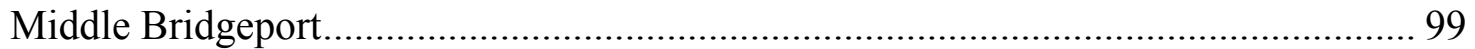

Transect 32 $3 \ldots \ldots \ldots$

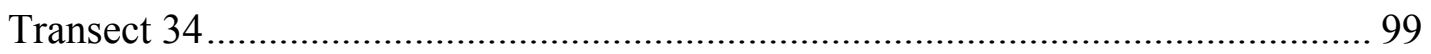

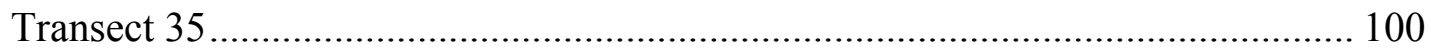

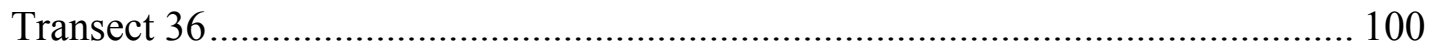

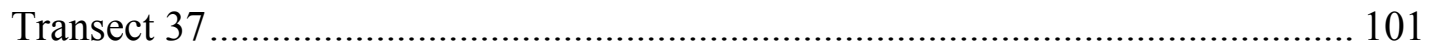

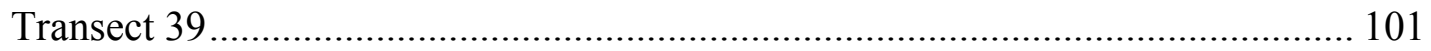

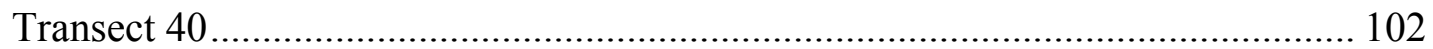

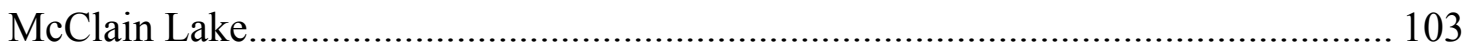

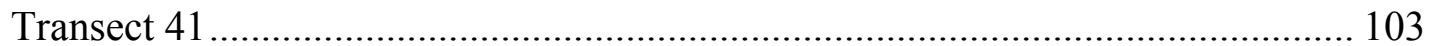

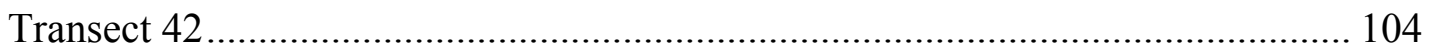

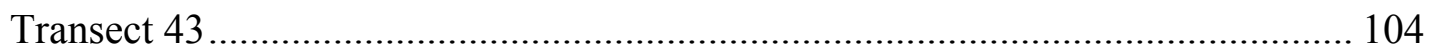

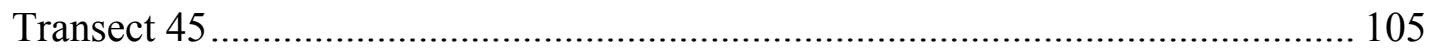

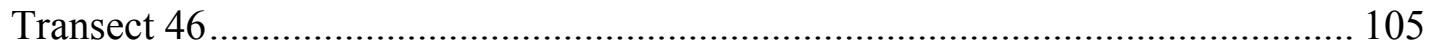

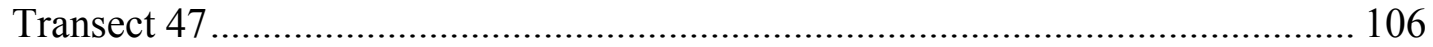

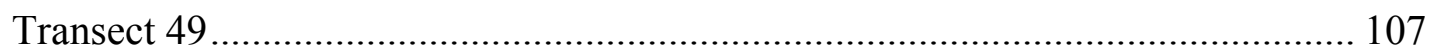

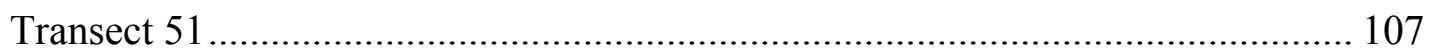

Transect 52

Transect 57

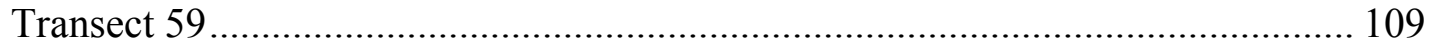

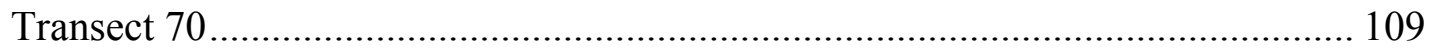

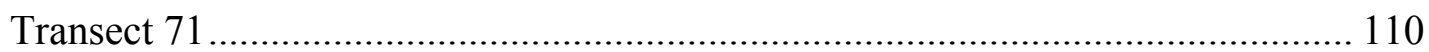

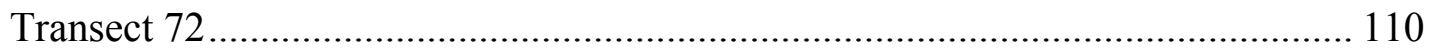

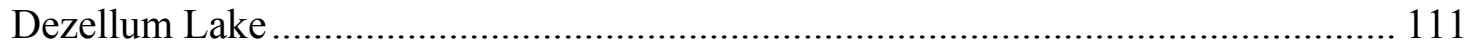

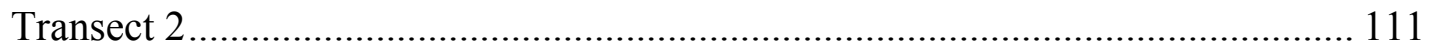


West Foster Creek Acquisitions

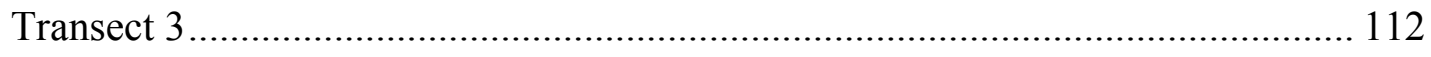

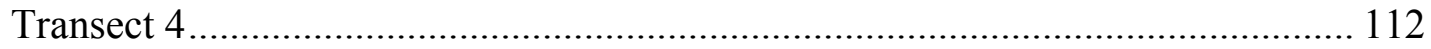

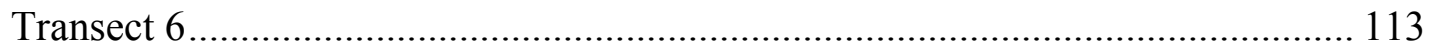

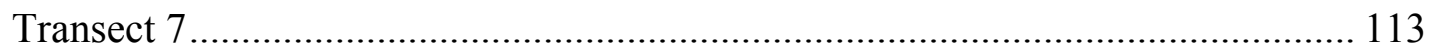

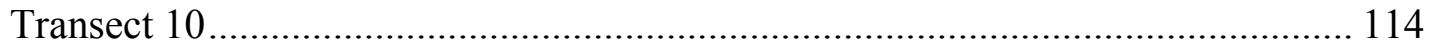

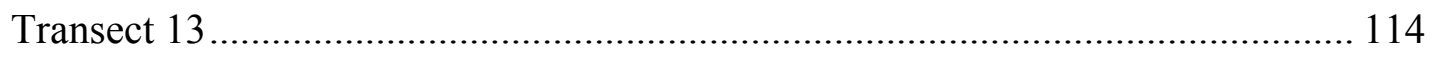

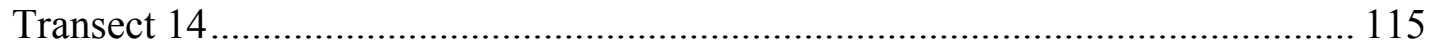

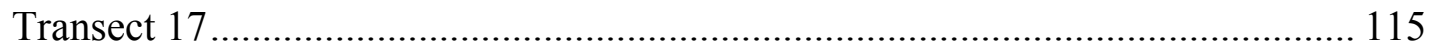

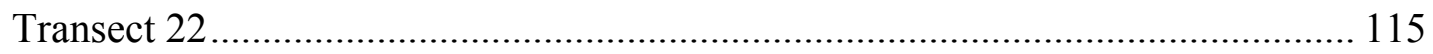

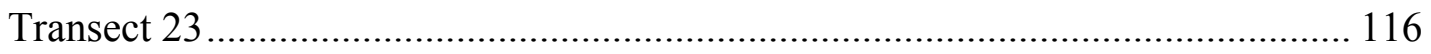

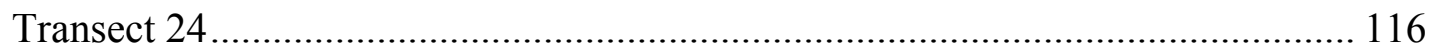

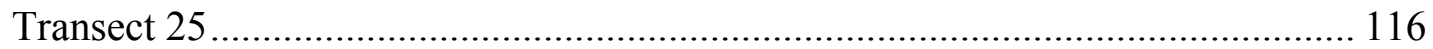

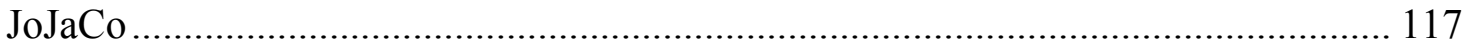

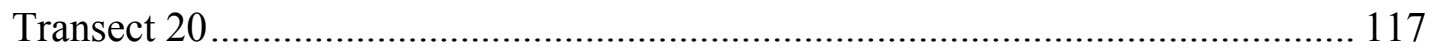

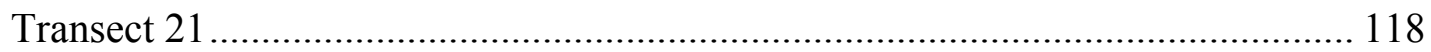

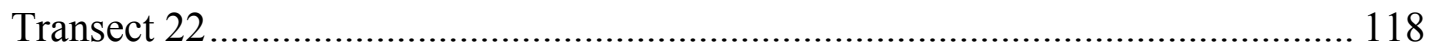

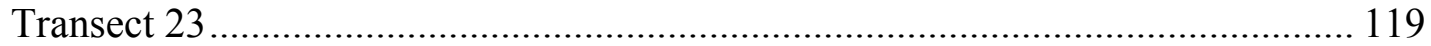

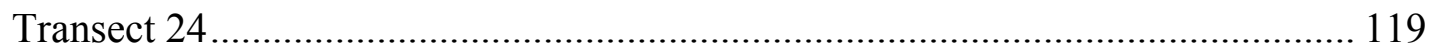

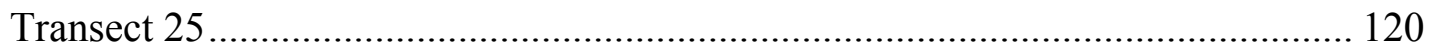

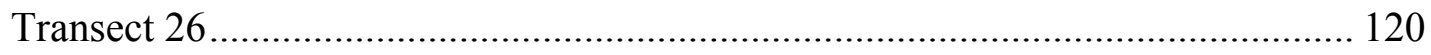

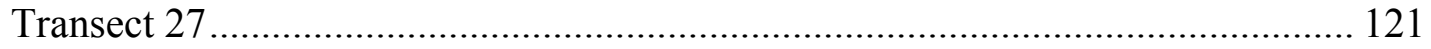

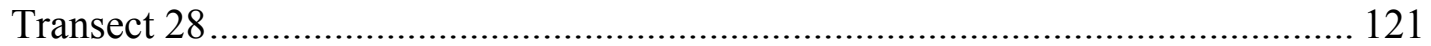

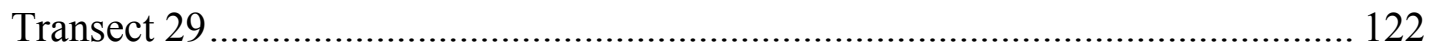

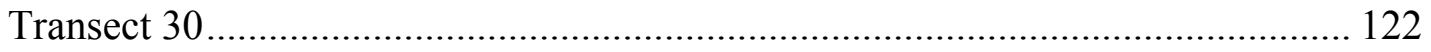

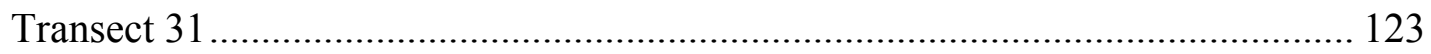

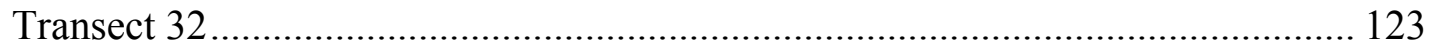

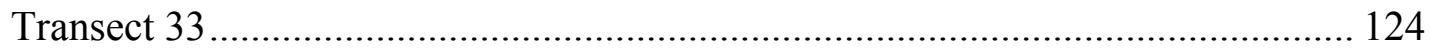

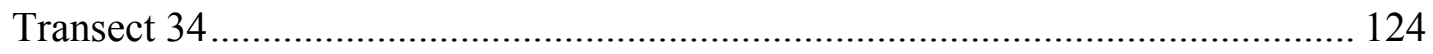

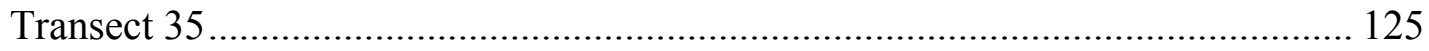

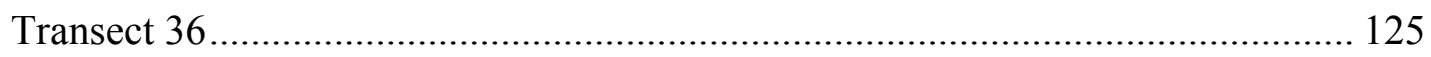

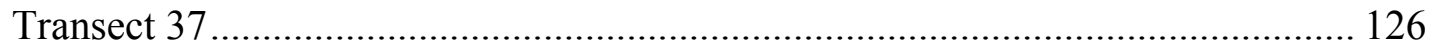

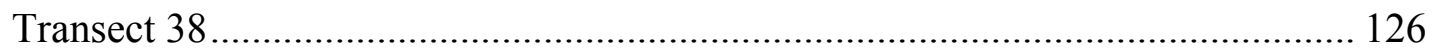

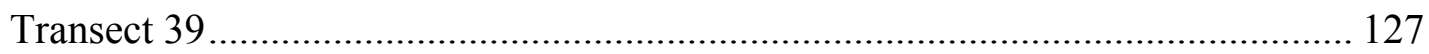

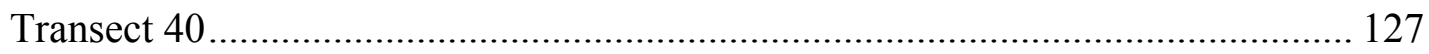

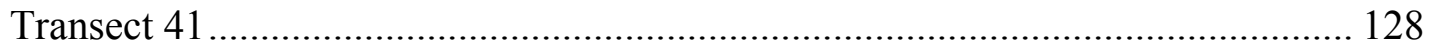

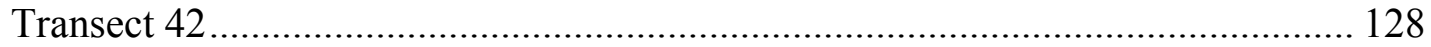

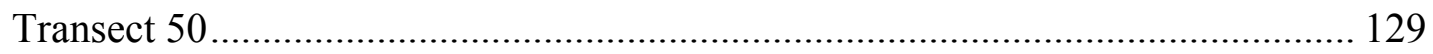

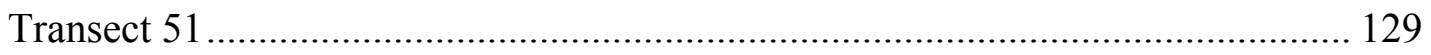

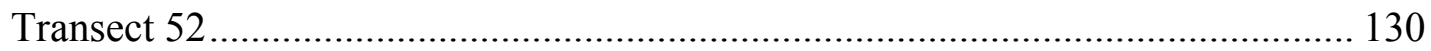

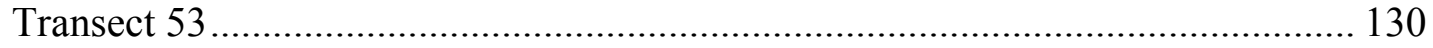

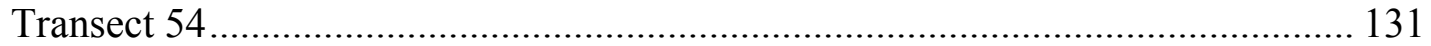

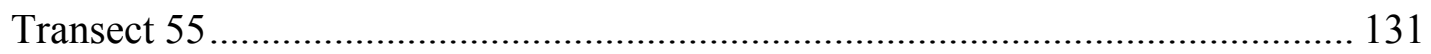




\section{Table of Figures}

Figure 1. West Foster Creek Acquisitions tract location map. ..................................... 3

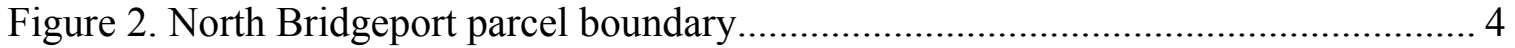

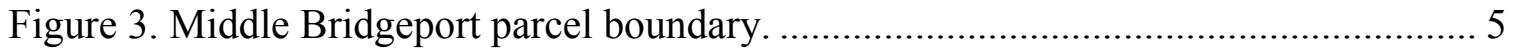

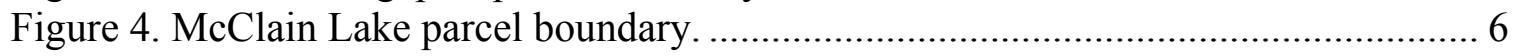

Figure 5. Dezellum Lake property boundary.............................................................. 7

Figure 6. JoJaCo Unit with individual parcel boundaries......................................... 8

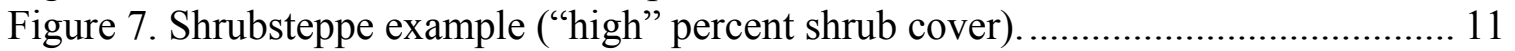

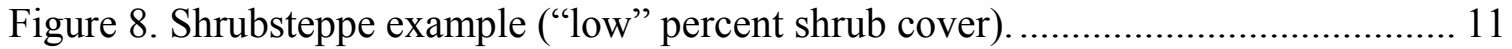

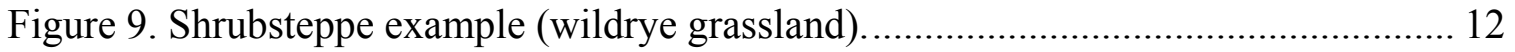

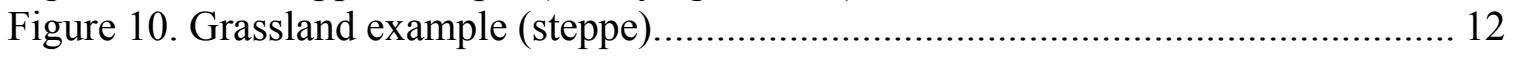

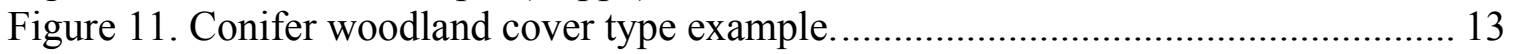

Figure 12. Rock/Cliff/Talus example. ...................................................................... 14

Figure 13. Deciduous tree and shrub cover type example (introduced poplar)............... 15

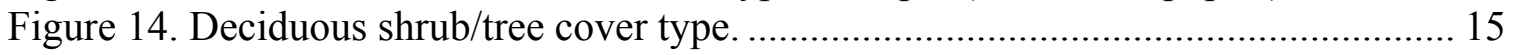

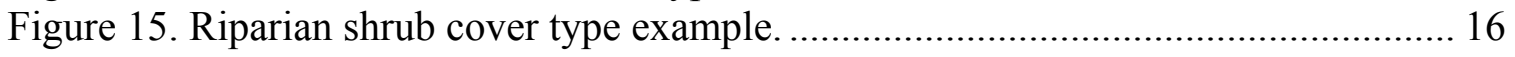

Figure 16. Riparian forest cover type example....................................................... 17

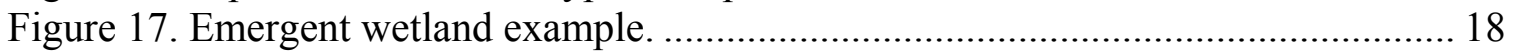

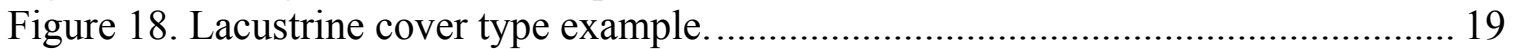

Figure 19. HEP data collection and processing flow chart......................................... 24

Figure 20. Transect photo point example. .............................................................. 30 
West Foster Creek Acquisitions

\section{List of Tables}

Table 1. West Foster Creek Expansion cover types, acres, and relative percent of area.... 9

Table 2. West Foster Creek Expansion cover type definitions.................................... 10

Table 3. Habitat suitability index verbal equivalency table........................................ 20

Table 4. West Foster Creek Expansion Project cover type/HEP species model matrix... 21

Table 5. HEP model species selection rationale table. ................................................ 22

Table 6. West Foster Creek Expansion project transect coordinates, azimuths, and

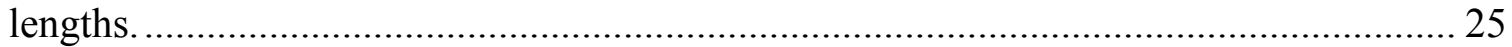

Table 7. North Bridgeport 2007 baseline HEP results summary.................................. 31

Table 8. Middle Bridgeport 2007 baseline HEP results summary................................. 32

Table 9. McClain Lake 2007 baseline HEP results summary. ....................................... 33

Table 10. Dezellum Lake 2007 Baseline HEP survey results summary.......................... 35

Table 11. JoJaCo 2007 baseline HEP survey results summary. ...................................... 37 


\section{Abstract}

During April and May 2007, the Columbia Basin Fish and Wildlife Authority's (CBFWA) Regional HEP Team (RHT) conducted baseline Habitat Evaluation Procedures (HEP) (USFWS 1980, 1980a) analyses on five parcels collectively designated the West Foster Creek Expansion Project (3,756.48 acres). The purpose of the HEP analyses was to document extant habitat conditions and to determine how many baseline/protection habitat units (HUs) to credit Bonneville Power Administration (BPA) for funding maintenance and enhancement activities on project lands as partial mitigation for habitat losses associated with construction of Grand Coulee and Chief Joseph Dams.

HEP evaluation models included mule deer (Odocoileus hemionus), western meadowlark (Sturnella neglecta), sharp-tailed grouse, (Tympanuchus phasianellus), Bobcat (Lynx rufus), mink (Neovison vison), mallard (Anas platyrhynchos), and black-capped chickadee (Parus atricapillus). Combined 2007 baseline HEP results show that 4,946.44 habitat units were generated on 3,756.48 acres (1.32 HUs per acre). HEP results/habitat conditions were generally similar for like cover types at all sites. Unlike crediting of habitat units (HUs) on other WDFW owned lands, Bonneville Power Administration received full credit for HUs generated on these sites.

\section{Introduction}

West Foster Creek Expansion Project (WFCEP) parcels are owned by Washington Department of Fish and Wildlife (WDFW). WDFW acquired the sites to protect critical sharp-tailed grouse (Tympanuchus phasianellus columbianus) and sage grouse (Centrocercus urophasianus) habitat and winter mule deer range (M. Schroeder, pers. comm.). M. Hallet (pers. comm.) stated that another reason WDFW acquired the sites was to increase public recreation opportunities in the local area.

West Foster Creek Expansion Project sites were purchased by WDFW with Washington Wildlife and Recreation Program (WWRP) funds in 2002 and 2005 (D. Budd, pers. comm.). The following three parcels were purchased in 2002:

1. Wilson (421.48 acres - $\$ 155,700)$

2. Gross $(147$ acres $-\$ 47,000)$

3. JoJaCo (826 acres $-\$ 295,000)$

WDFW purchased 2,362 acres from the Dezellum family in 2005 (10/25/05) for $\$ 839,000$ (D. Budd, pers. comm.). Individual parcels included:
1. North Bridgeport
2. Middle Bridgeport
3. Dezellum Lake
4. Dezellum East
5. McClain Lake 
The Wilson, Gross, JoJaCo, and the Dezellum East ${ }^{1}$ parcels were combined and evaluated as a single unit by the Regional HEP Team, which also designated the combined sites "JoJaCo" for the 2007 HEP surveys.

Unlike crediting of habitat units (HUs) on other WDFW owned lands, Bonneville Power Administration received full credit for HUs generated on these sites. This change in crediting protocols occurred to fulfill WDFW's commitment to make BPA whole for withdrawing the Cleman Mountain Unit (Wenas Wildlife Area) and related HUs from the wildlife mitigation program and to compensate BPA for maintenance and enhancement expenditures and Washington Department of Natural Resource (DNR) land lease payments associated with the Cleman Mountain Unit.

Due to the similarity and close juxtaposition of the sites, this report includes details and results of HEP analyses for all five parcels i.e., North Bridgeport, Middle Bridgeport, McClain Lake, Dezellum Lake, and JoJaCo. General information such as cover type definitions, HEP models, and transect methods are described once in this report while HEP transect results, parcel cover type information, transect locations, and photo points are dealt with separately for each parcel. The 2007 HEP surveys were established using the same transect protocols and measurement techniques used to complete the 1999 baseline HEP surveys on nearby parcels (WDFW 2001).

\footnotetext{
${ }^{1}$ Dezellum East was purchased by WDFW in 2005. The Wilson, Gross, and JoJaCo sites were acquired in 2002.
} 


\section{Study Area}

\section{Location}

The five WFCEP parcels are located within five miles of Bridgeport, Washington and Chief Joseph Dam (Figure 1) (UTM: 11 0300859E, 5320461N). Tracts ${ }^{2}$ range in size from approximately 162 acres to 2,638 acres and included the following individual sites:

1. North Bridgeport (320.50 acres)

2. Middle Bridgeport (161.90 acres)

3. McClain Lake (469.19 acres)

4. Dezellum Lake (166.58 acres)

5. $\mathrm{JoJaCo}^{3}\left(2,638.31\right.$ acres $\left.^{4}\right)$

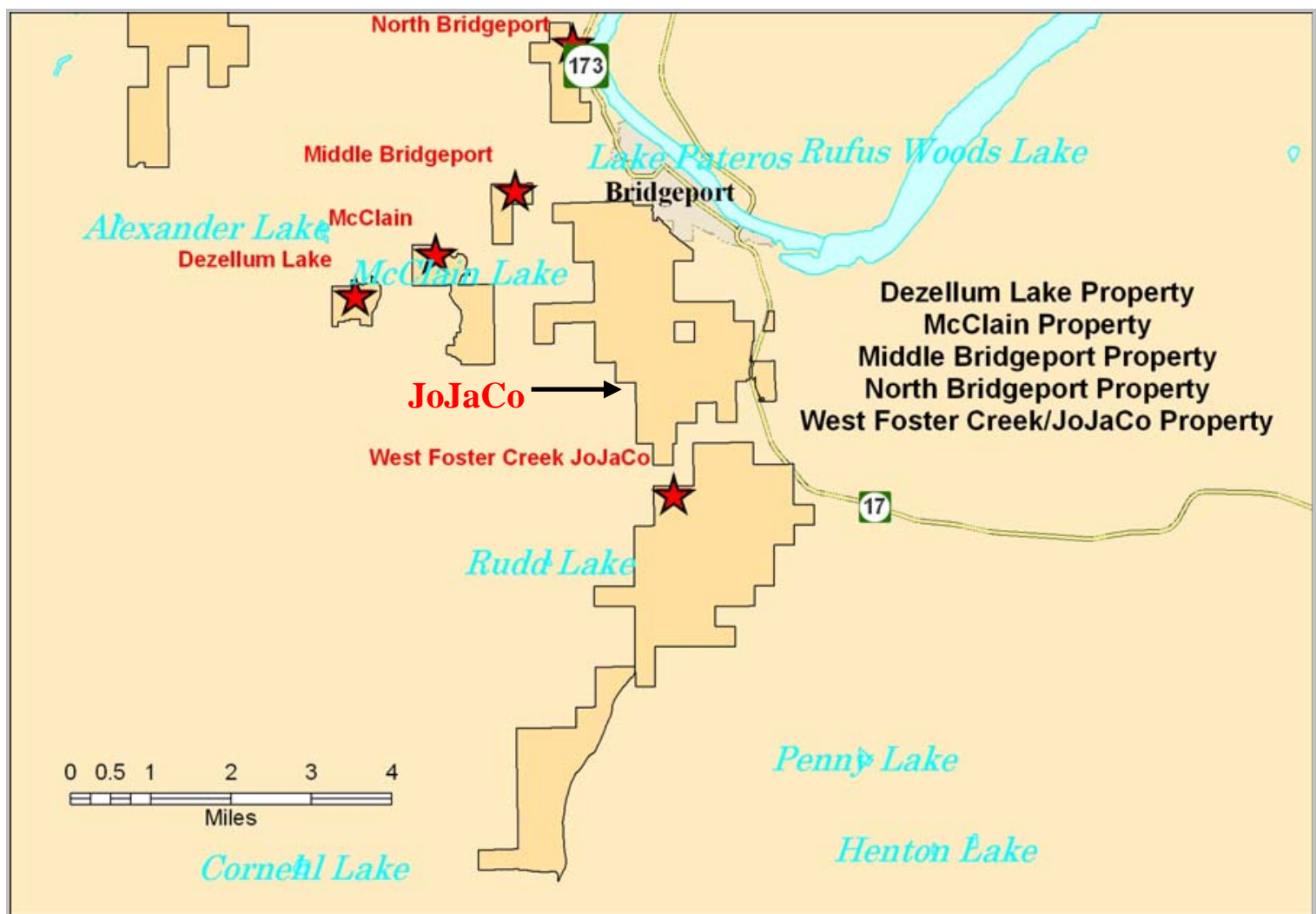

Figure 1. West Foster Creek Acquisitions tract location map.

Property boundaries are illustrated in Figure 2 through Figure 6. The JoJaCo, Wilson, Gross, and Dezellum East sites were combined and designated "JoJaCo" for purposes of the 2007 baseline HEP analysis (Figure 6).

\footnotetext{
${ }^{2}$ Parcels were named by the RHT to facilitate data collection/separation and may not be the same designations used by WDFW to identify the sites.

${ }^{3}$ The JoJaCo parcel is the tract located immediately south of the word "Bridgeport" in Figure 1. WDFW's designation for this site was "Wilson-JoJaCo. The RHT shortened the name to JoJaCo.

${ }^{4}$ Acreage includes the Dezellum East, Wilson, Gross, and JoJaCo properties.
} 
West Foster Creek Expansion Project

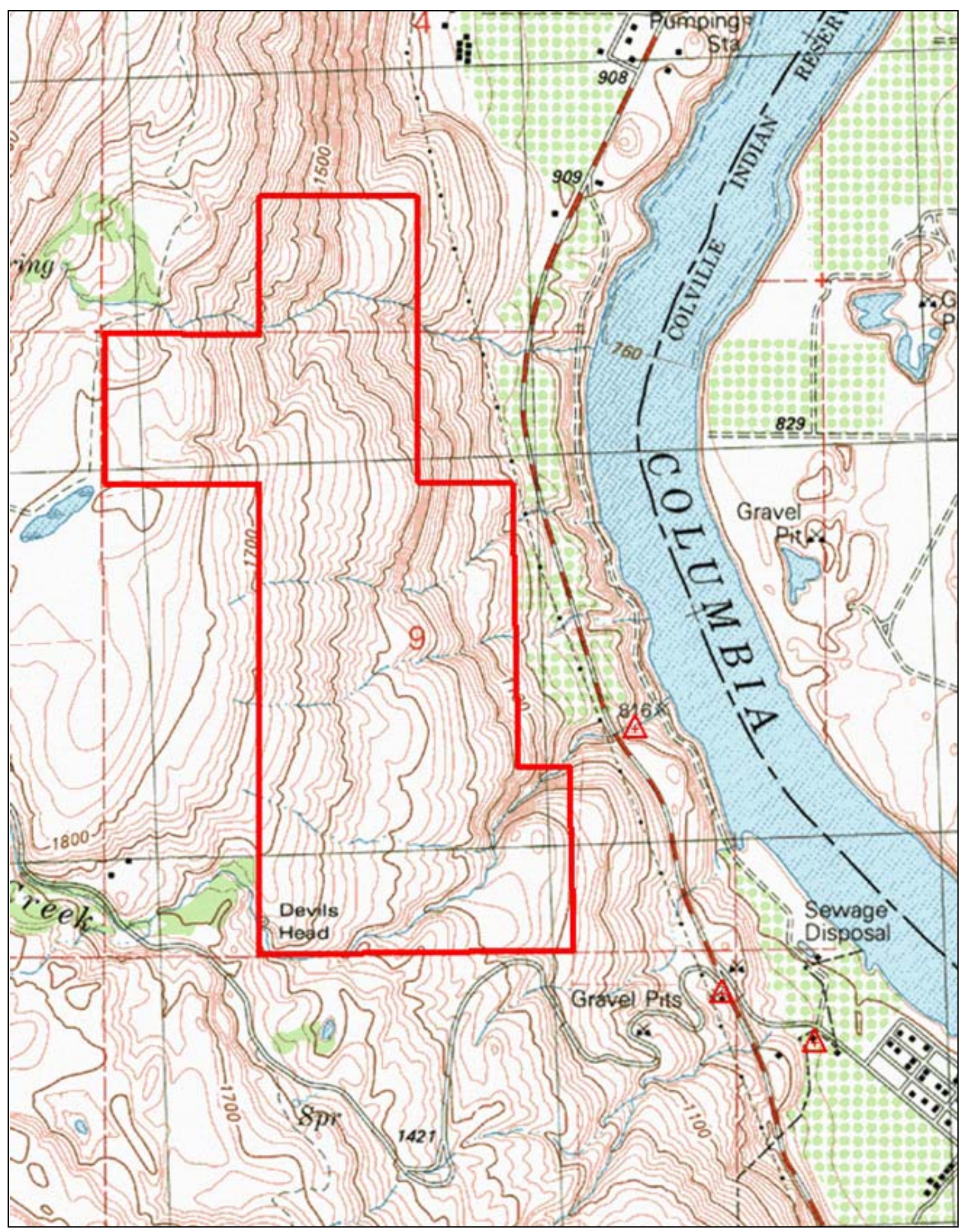

Figure 2. North Bridgeport parcel boundary. 
West Foster Creek Expansion Project

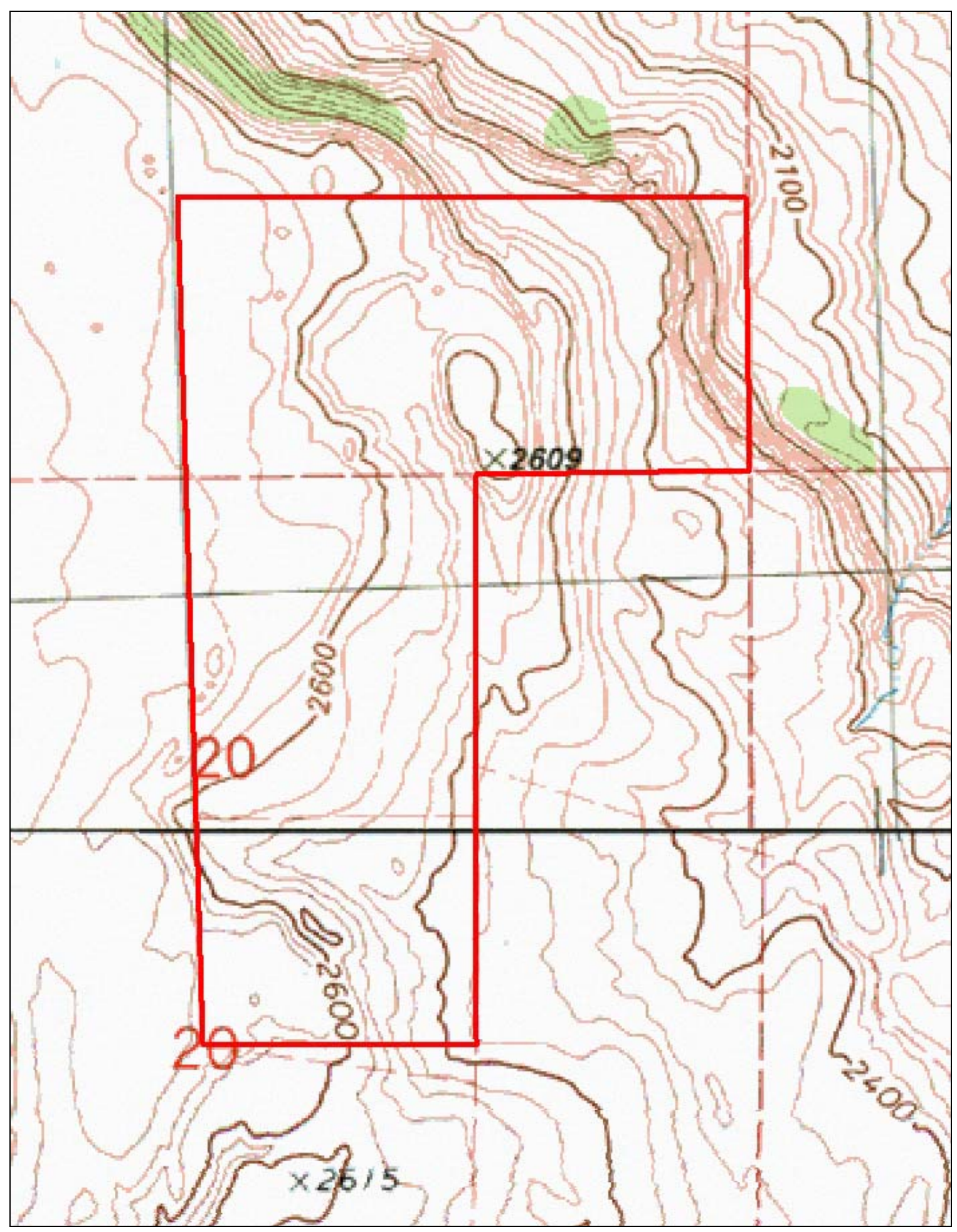

Figure 3. Middle Bridgeport parcel boundary. 
West Foster Creek Expansion Project

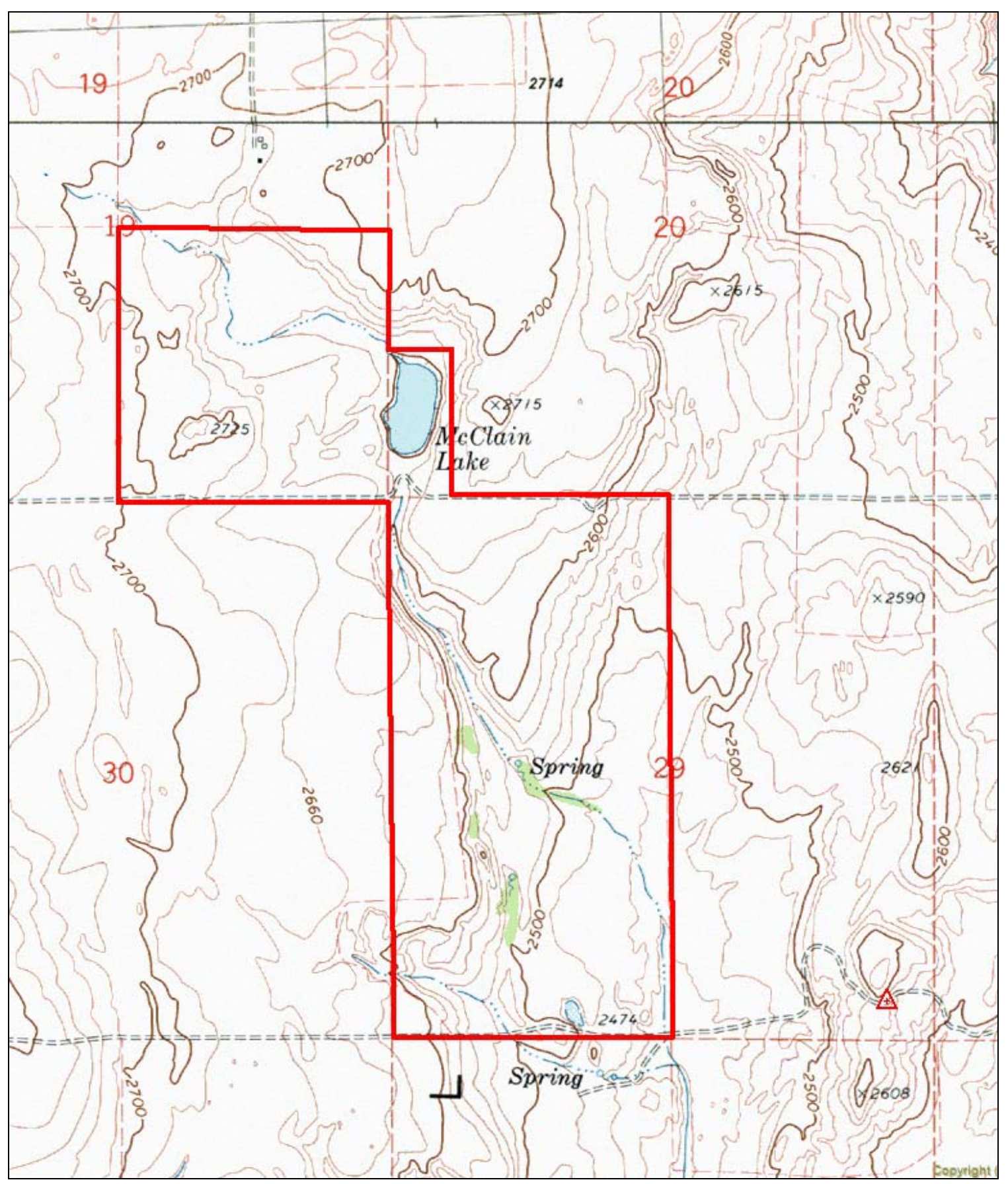

Figure 4. McClain Lake parcel boundary. 
West Foster Creek Expansion Project

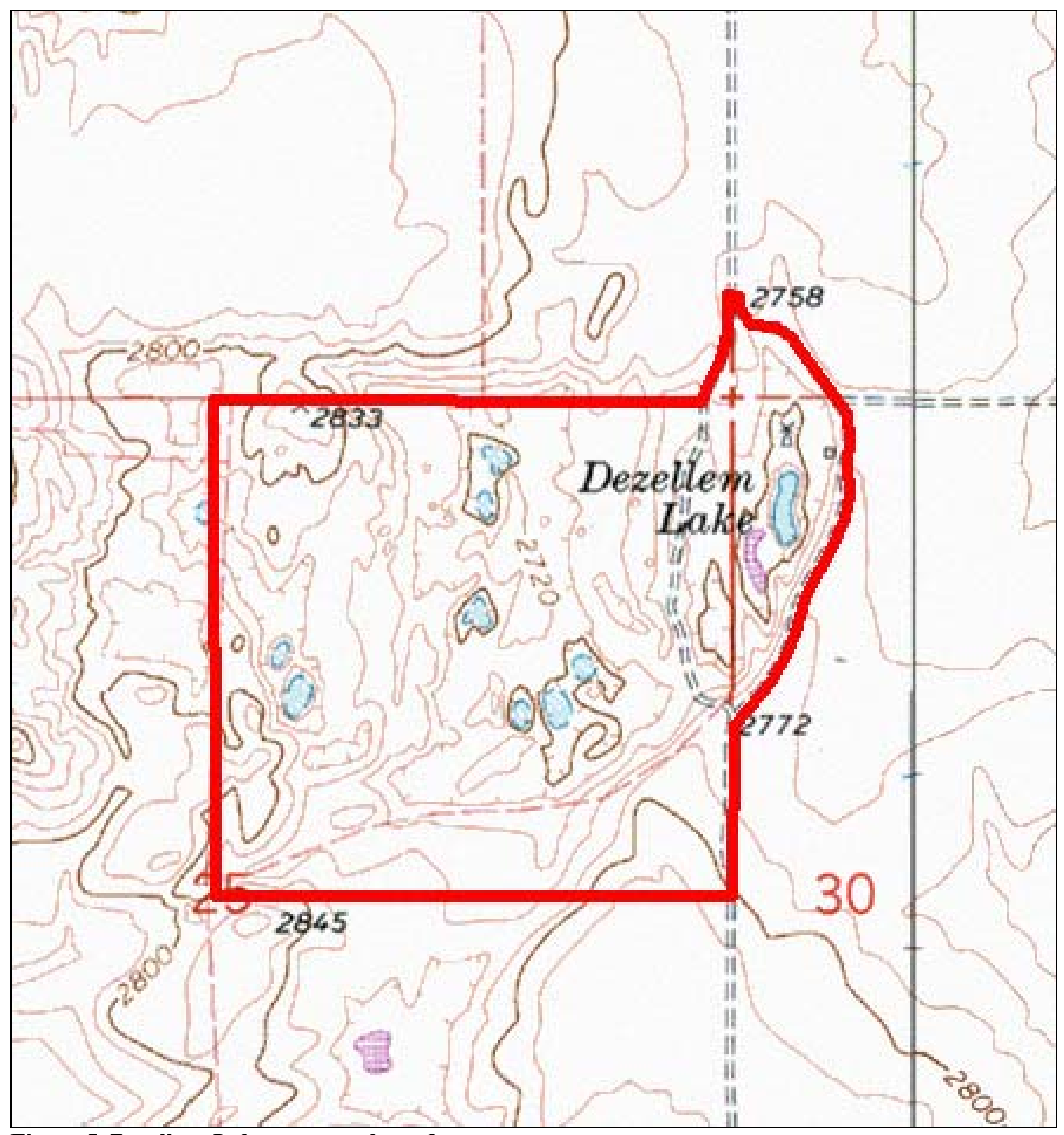

Figure 5. Dezellum Lake property boundary. 


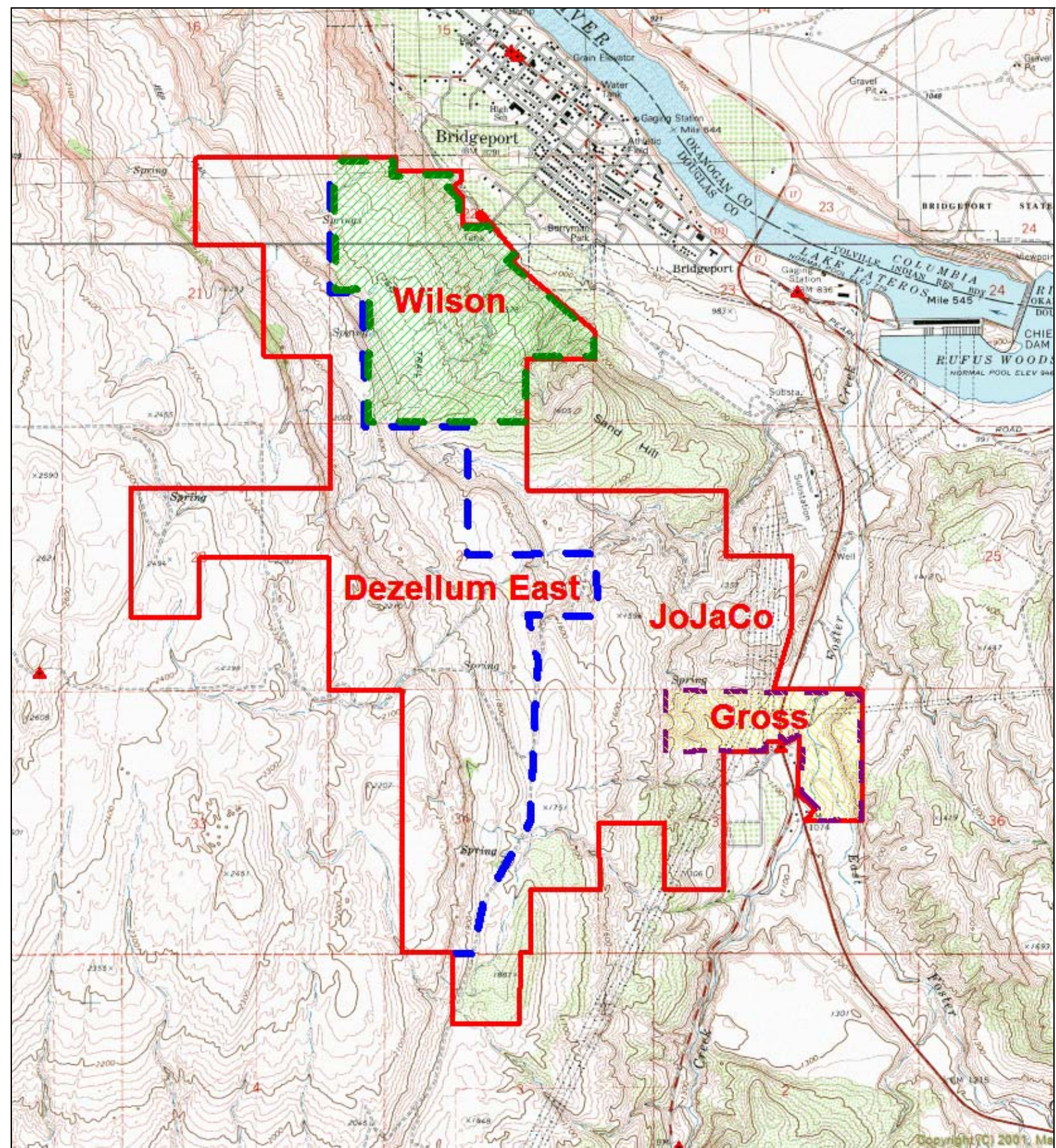

Figure 6. JoJaCo Unit with individual parcel boundaries.

\section{Topography}

Elevation ranges from approximately 1,100 feet on the Bridgeport North parcel to nearly 2,800 feet at Dezellum Lake. Topography varies from flat pasture and rolling hills to incised stream channels and mountainous terrain dominated by rock outcrops (Maptech Terrain Navigator Pro (®) software). 


\section{Cover Types}

Cover type maps were not available prior to initiation of the HEP analyses. Therefore, RHT staff developed coarse cover type maps from aerial photographs and "ground-truthed" the maps while conducting HEP surveys. To maintain consistency with previous HEP analyses on adjacent lands, the Regional HEP Team did not divide shrubsteppe habitat into sub-cover types as is done on GAP vegetation class maps (Ohmann et. al. 2006) currently used by WDFW (J. Talmadge, pers. comm.).

Nearly $98 \%(3,670.15$ acres $)$ of the total 3,756.48 acres that comprise the West Foster Creek Expansion Project was shrubsteppe (shrubsteppe includes grassland except at the McClain Lake parcel). The remaining 2\% (86.33 acres) was divided between deciduous shrub/tree, lacustrine, emergent wetland, riparian shrub, riparian forest, conifer forest, and rock/cliff/talus cover types (Table 1). WFCEP cover types are defined in Table 2.

Table 1. West Foster Creek Expansion cover types, acres, and relative percent of area.

\begin{tabular}{|c|c|c|c|}
\hline Parcel & Cover Type & Acres & Percent of Area \\
\hline \multirow{2}{*}{ North Bridgeport } & Shrubsteppe ${ }^{1}$ & 314.10 & $98.00 \%$ \\
\hline & Deciduous Shrub/Tree & 6.40 & $2.00 \%$ \\
\hline Total & & 320.50 & $100.00 \%$ \\
\hline Middle Bridgeport & Shrubsteppe $^{1}$ & 161.90 & $100.00 \%$ \\
\hline Total & & 161.90 & $100.00 \%$ \\
\hline \multirow{5}{*}{ McClain Lake } & Shrubsteppe (shrubland) & 354.26 & $75.50 \%$ \\
\hline & Grassland & 90.00 & $19.18 \%$ \\
\hline & Deciduous Shrub/Tree & 14.50 & $3.09 \%$ \\
\hline & Lacustrine & 9.76 & $2.08 \%$ \\
\hline & Emergent Wetland & 0.67 & $0.14 \%$ \\
\hline Total & & 469.19 & $100.00 \%$ \\
\hline \multirow{3}{*}{ Dezellum Lake } & Shrubsteppe ${ }^{1}$ & 156.58 & $94.00 \%$ \\
\hline & Emergent Wetland & 5.00 & $3.00 \%$ \\
\hline & Lacustrine & 5.00 & $3.00 \%$ \\
\hline Total & & 166.58 & $100.00 \%$ \\
\hline \multirow{5}{*}{ JoJaCo } & Shrubsteppe ${ }^{1}$ & $2,593.31$ & $98.30 \%$ \\
\hline & Riparian Shrub & 8.00 & $0.30 \%$ \\
\hline & Riparian Forest & 22.00 & $0.83 \%$ \\
\hline & Conifer Woodland & 7.00 & $0.27 \%$ \\
\hline & Rock/Cliff/Talus & 8.00 & $0.30 \%$ \\
\hline Total & & $2,638.31$ & $100.00 \%$ \\
\hline Grand Total & & $3,756.48$ & N/A \\
\hline
\end{tabular}


Table 2. West Foster Creek Expansion cover type definitions.

\begin{tabular}{|c|c|}
\hline Cover Type & Definition \\
\hline Shrubsteppe (shrubland) & $\begin{array}{l}\text { Xeric uplands dominated by shrubsteppe vegetation (includes native and } \\
\text { introduced plant species). Shrub cover is } \geq 5 \% \text {; Tree cover is }<5 \% \text {. }\end{array}$ \\
\hline Grassland (steppe) & $\begin{array}{l}\text { Generally, upland sites dominated by herbaceous vegetation with }<5 \% \text { shrub } \\
\text { or tree cover. }\end{array}$ \\
\hline Conifer Woodland & Xeric uplands dominated by ponderosa pine trees ( $\geq 5 \%$ tree canopy cover). \\
\hline Rock/Cliff/Talus & Areas dominated by rock. \\
\hline Deciduous Shrub/Tree & $\begin{array}{l}\text { Areas comprised of } \geq 5 \% \text { deciduous tree and/or macrophyllus shrub cover } \\
\text { without open water present. }\end{array}$ \\
\hline Riparian Shrub & Mesic areas dominated by hydrophytic/macrophyllus shrubs. \\
\hline Riparian Forest & $\begin{array}{l}\text { Mesic areas dominated by hydrophytic/deciduous trees with open water } \\
\text { present. }\end{array}$ \\
\hline Emergent Wetland & $\begin{array}{l}\text { Wetland sites with } \geq 5 \% \text { of the area supporting emergent vegetation e.g., } \\
\text { cattail, rush, sedge, etc. along shoreline and/or extending into water; } \\
\text { shrub/tree cover }<5 \% \text {. }\end{array}$ \\
\hline Lacustrine $^{1}$ & $\begin{array}{l}\text { Open water sites with }<5 \% \text { of the area comprised of emergent vegetation; } \\
\text { shrub/tree cover }<5 \% \text {. }\end{array}$ \\
\hline
\end{tabular}

\section{Cover Type Floristics}

Cover type floristics are briefly described in the following section. Raw transect data can be viewed by following the hyperlinks listed in the "Results Section."

\section{Shrubsteppe}

Shrubsteppe is comprised of both shrub and grassland components (except where previously noted). Shrub species ${ }^{5}$ detected on WFCEP sites included big sagebrush (Artemisia tridentata), three-tip sagebrush (A. tripartite), bitterbrush (Purshia tridentata), green rabbitbrush

(Ericameria viscidiflorus), gray rabbitbrush (E. nauseosa) rose (Rosa spp.), shrubby penstemon (Penstemon fruticosus), and currant (Ribes spp.).

The herbaceous stratum consisted of both native and introduced species. Grass and forbs species observed included bluebunch wheatgrass (Pseudoroegneria spicata), Sandberg bluegrass (Poa secunda), needle-and- thread (Stipa comata), bottlebrush squirrel tail (Sitanion hystrix), Basin wildrye (Leymus cinereus), Indian ricegrass (Achnatherum hymenoides), lupine (Lupinus spp.), balsam root (Balsamorhiza sagittata), yarrow (Achillea millefolium), vetch (Astragalus spp.), cheatgrass (Bromus tectorum), crested wheatgrass (Agropyron cristatum), and mustard (Brassica spp.) to name a few. Examples of shrubsteppe are shown in Figure 7 through Figure 10.

\footnotetext{
${ }^{5}$ This is a composite shrub list for all sites. Not all shrub species occurred on all transects or at all sites.
} 
West Foster Creek Expansion Project

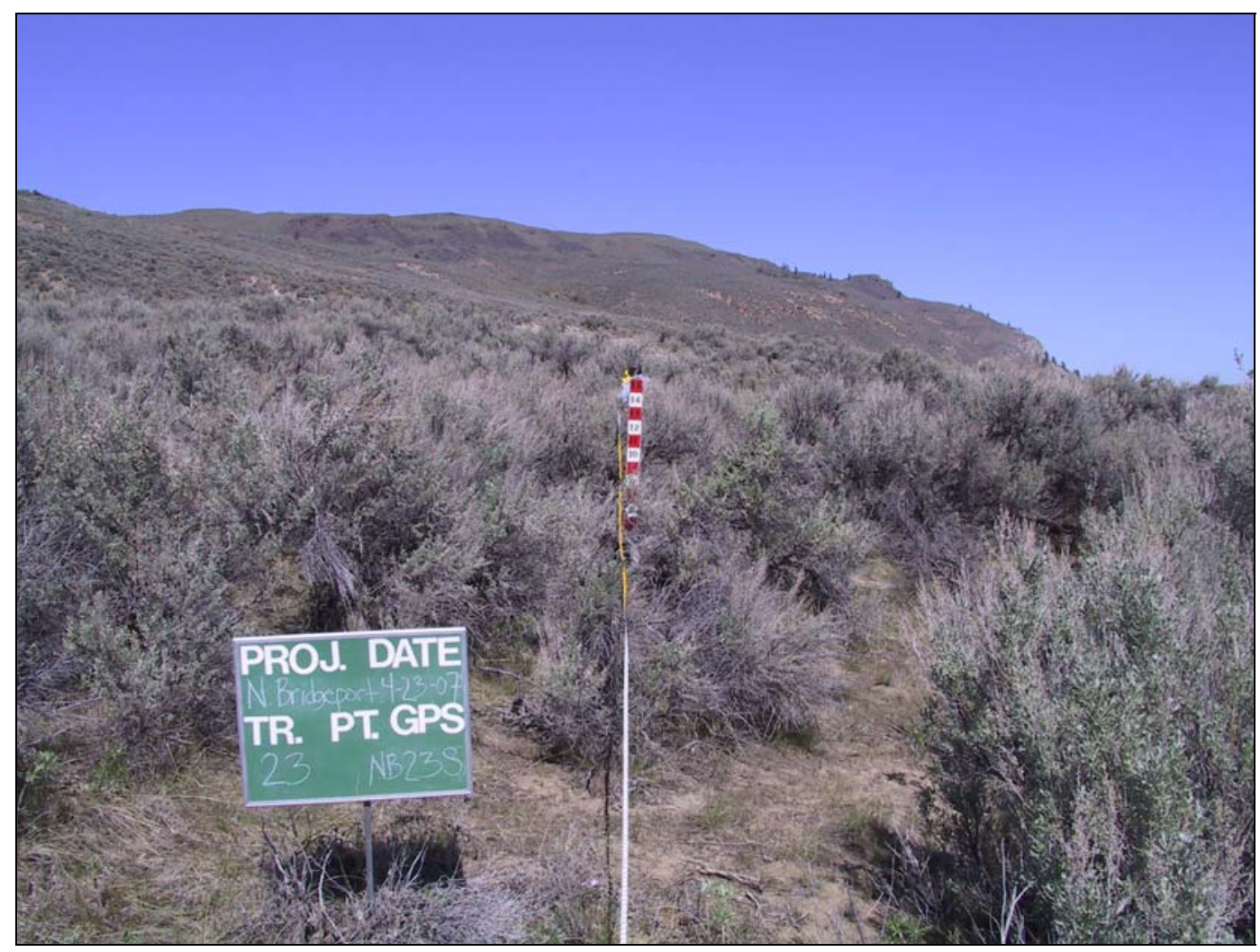

Figure 7. Shrubsteppe example ("high” percent shrub cover).

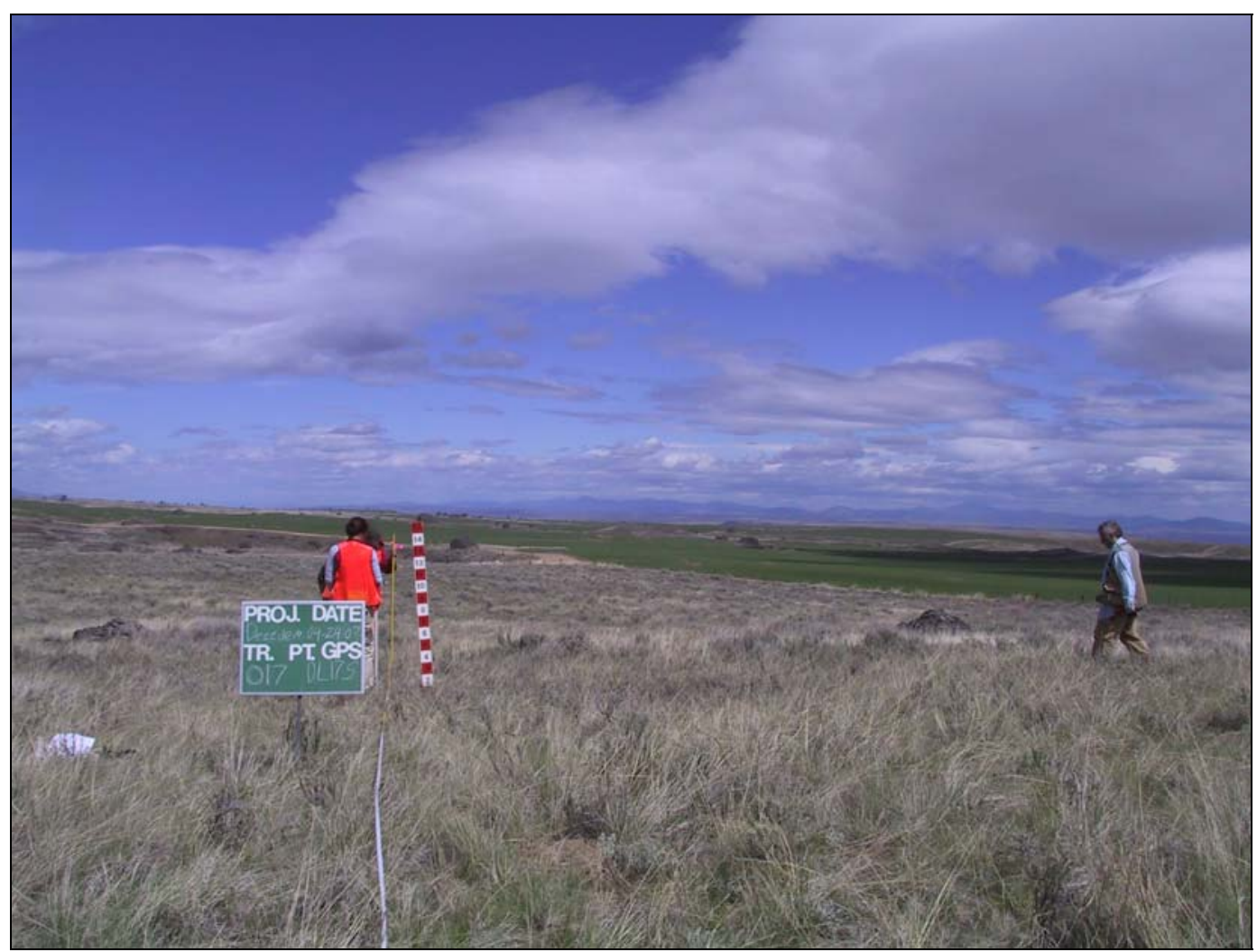

Figure 8. Shrubsteppe example (“low” percent shrub cover). 
West Foster Creek Expansion Project

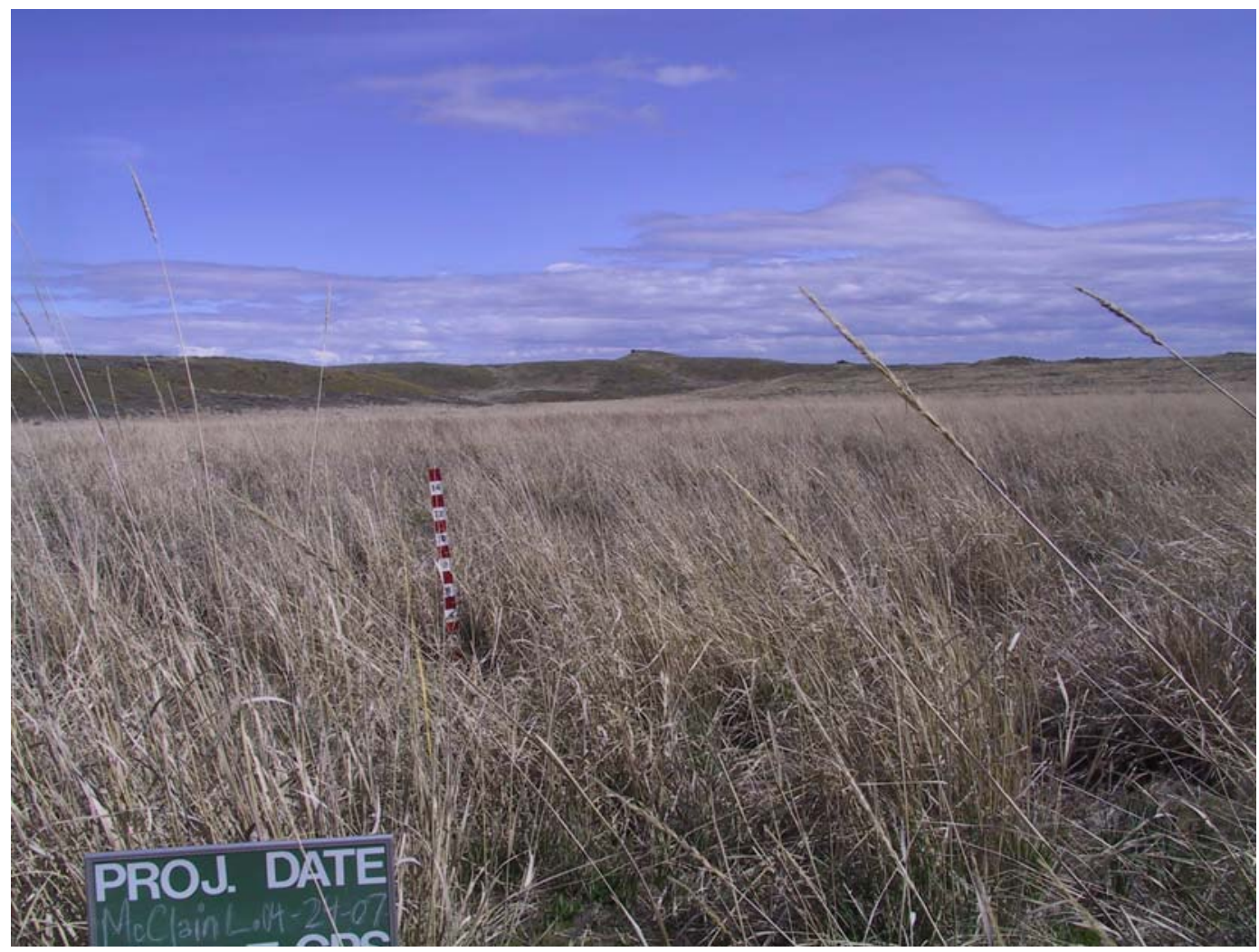

Figure 9. Shrubsteppe example (wildrye grassland).

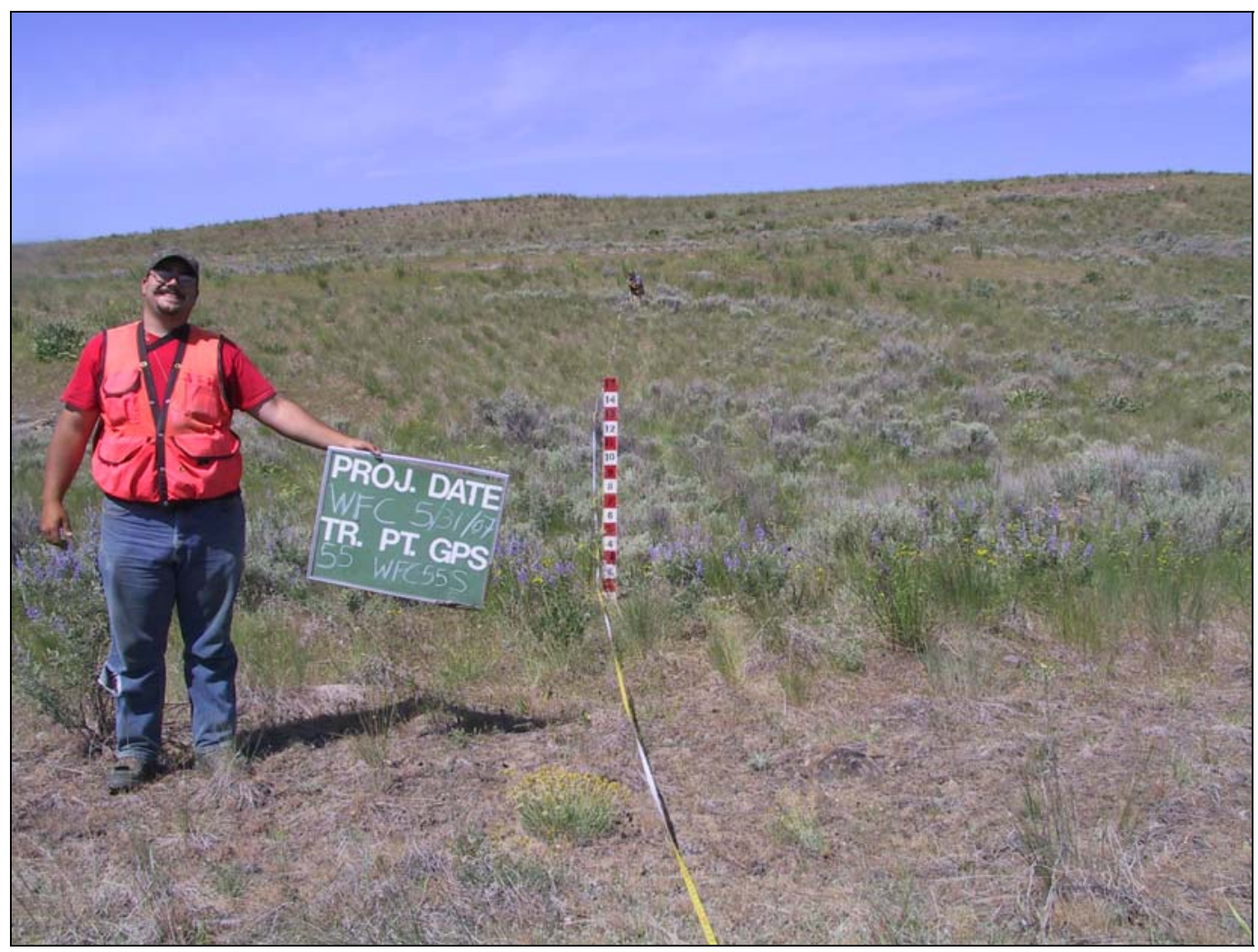

Figure 10. Grassland example (steppe). 


\section{Conifer Woodland}

The conifer woodland cover type was located primarily on the JoJaCo parcel. Ponderosa pine (Pinus ponderosa) trees were the only tree species detected. The shrub stratum was dominated by serviceberry (Amelanchier alnifolia) at slightly under $11 \%$ cover while wax currant (Ribes cereum) and big sagebrush were present in trace amounts $(<1 \%$ cover $)$.

Total herbaceous cover was $\approx 51 \%$ comprised primarily of native grasses; percent forbs cover was $4.9 \%$. In contrast, introduced/exotic herbaceous species were not detected. The conifer woodland cover type is illustrated in Figure 11.

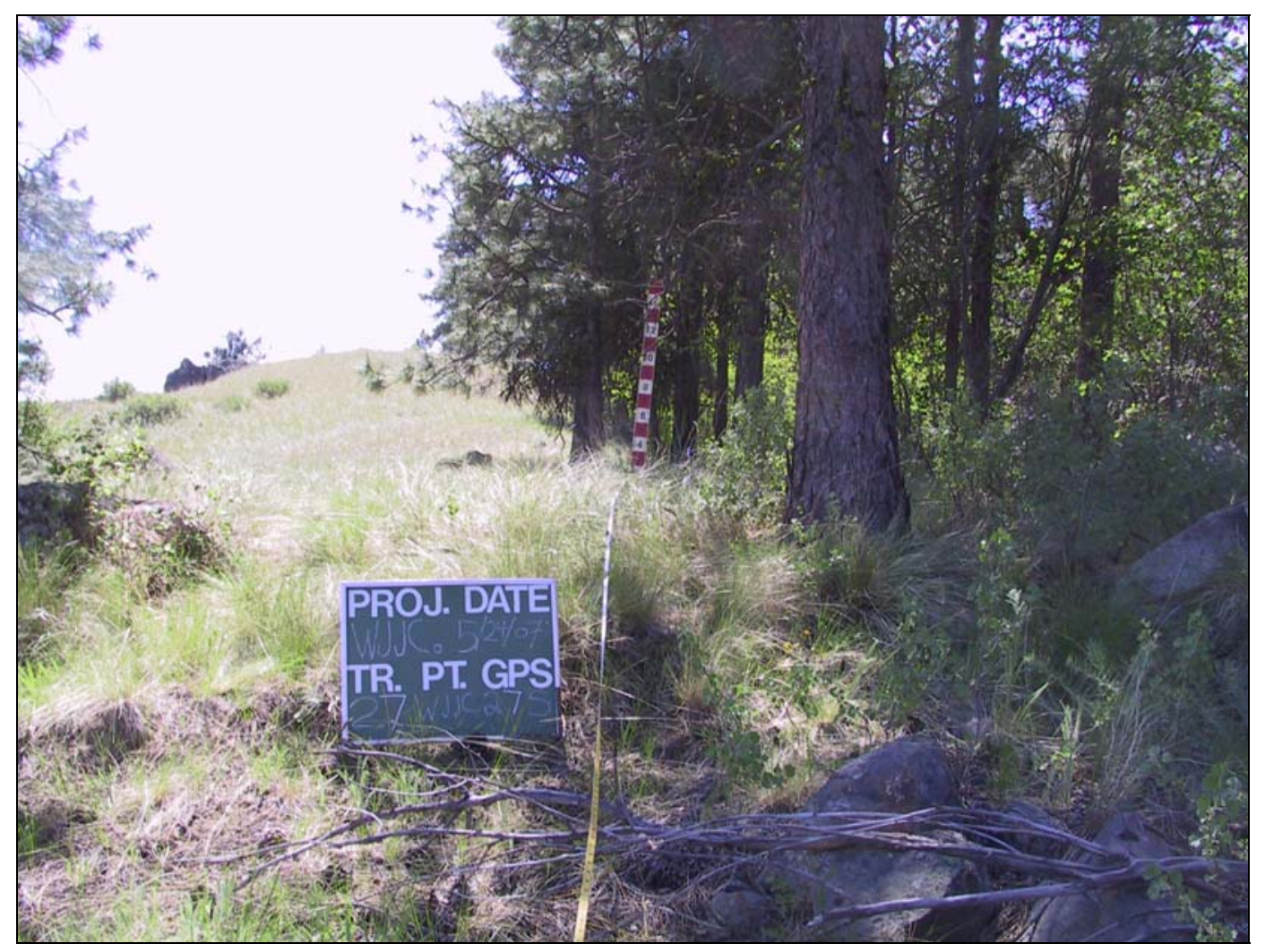

Figure 11. Conifer woodland cover type example.

\section{Rock/Cliff/Talus}

This cover type was dominated by rock cliffs and talus slopes as shown in Figure 12. Deciduous shrubs and pockets of herbaceous vegetation were interspersed throughout the cover type. 


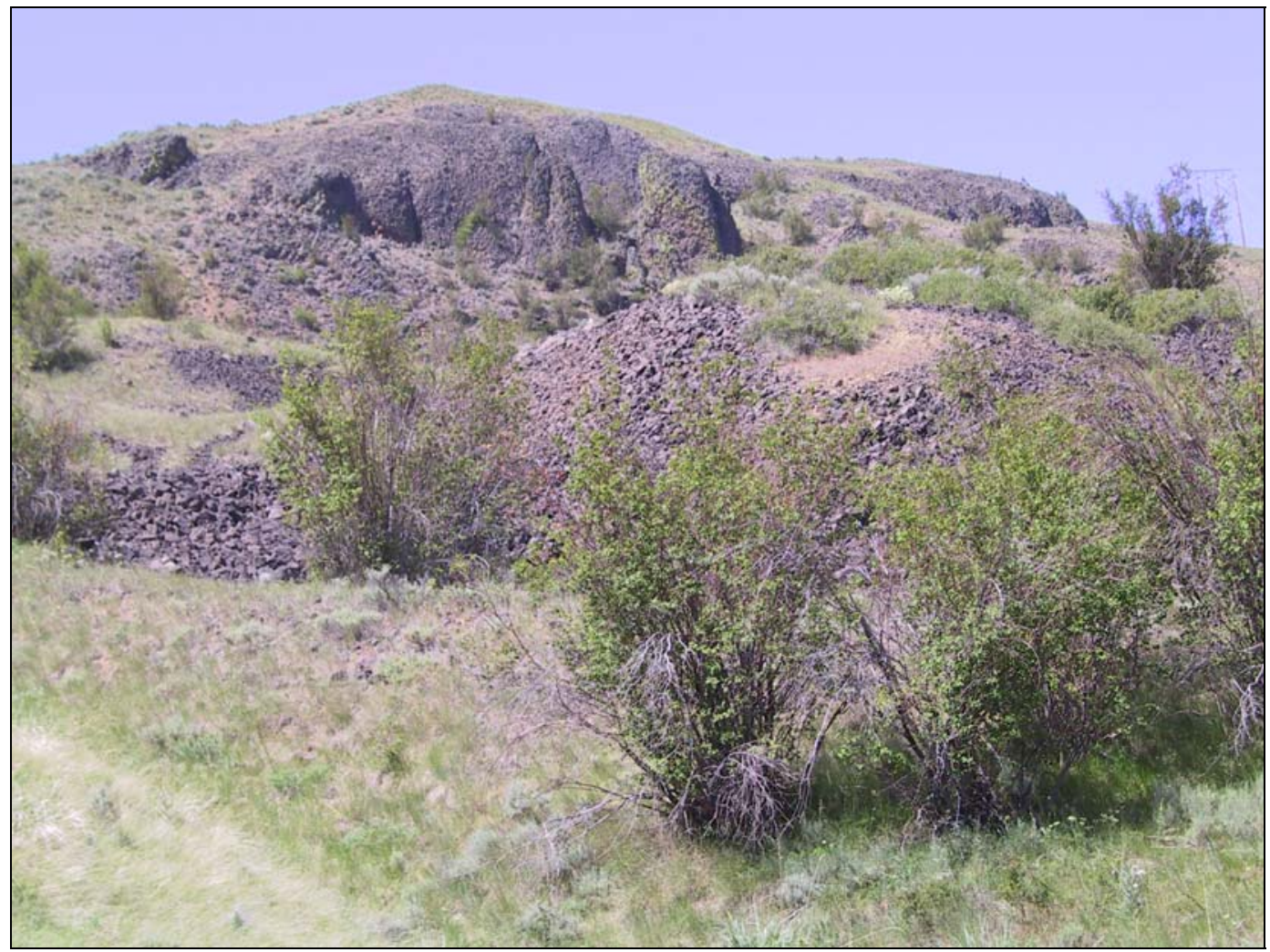

Figure 12. Rock/Cliff/Talus example.

\section{Deciduous Shrub/Tree}

The deciduous shrub/tree cover type was dominated by introduced tree and shrub ${ }^{6}$ species on some sites e.g., silver poplar (Populus spp.), and native shrubs and trees on other sites. Native trees and shrubs included quaking aspen (P. tremuloides), rose (Rosa spp.), and dogwood (Cornus sericea). Although not deciduous, big sagebrush was also present.

Poplar trees appeared "stressed" due likely to recent drought conditions and insect damage. Similarly, aspen stands also appeared to be in a stressed condition. Snags were abundant and predominantly $\leq 4$ inches diameter breast height (DBH). This cover type is depicted in Figure 13 and Figure 14.

\footnotetext{
${ }^{6}$ Shrubs included tree species $\leq 16$ feet in height (from a wildlife perspective, small trees generally function more like shrubs than mature trees).
} 
West Foster Creek Expansion Project

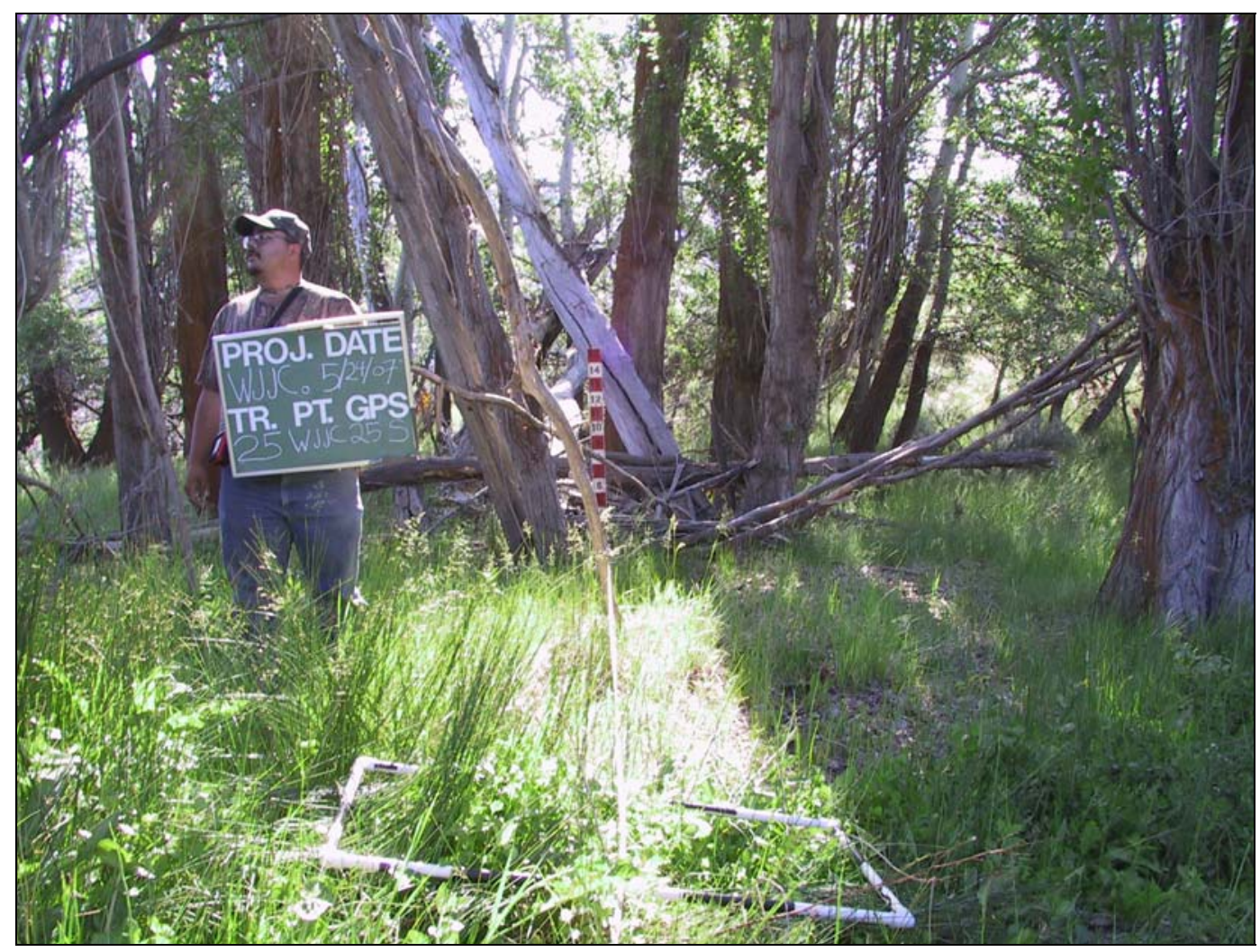

Figure 13. Deciduous tree and shrub cover type example (introduced poplar).

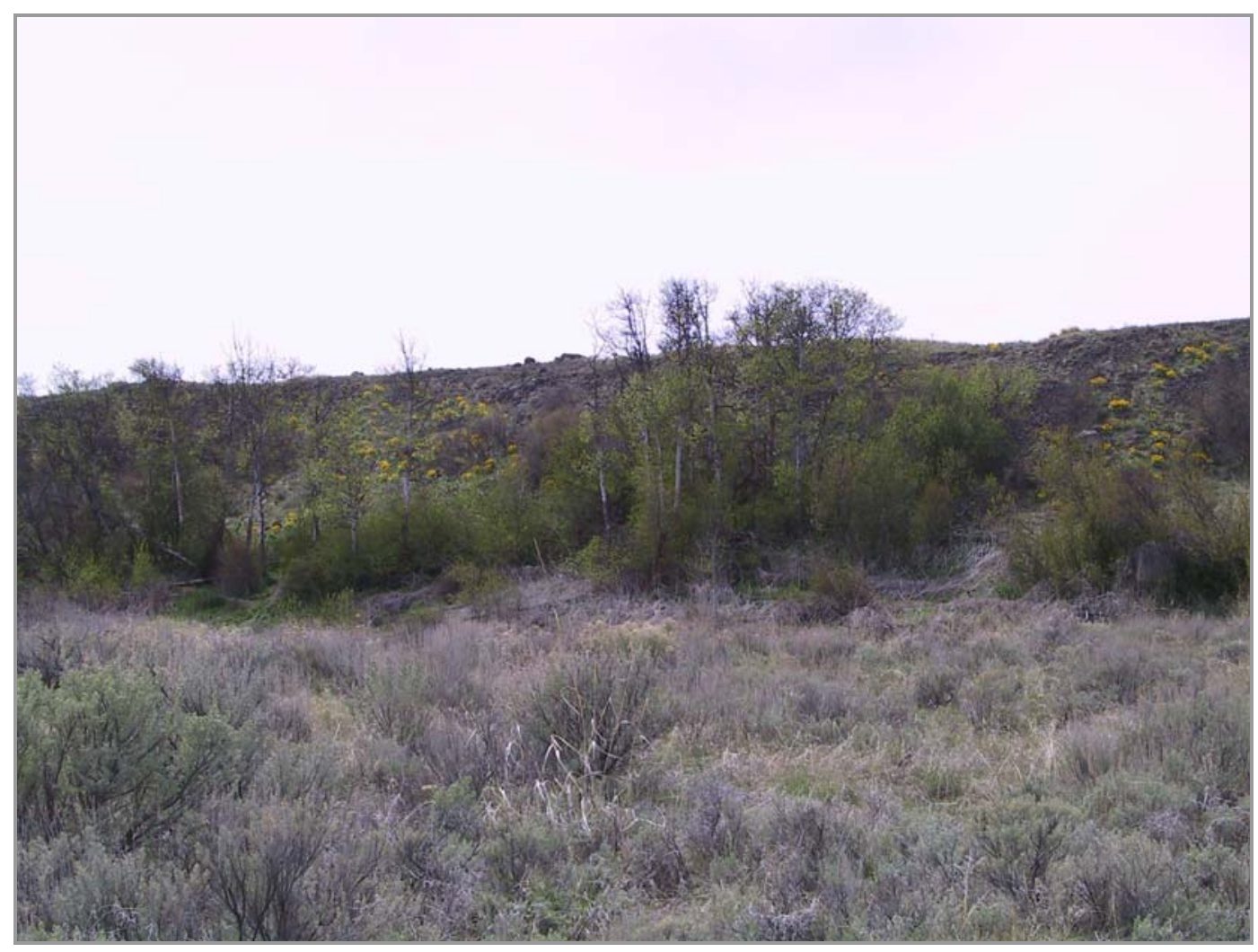

Figure 14. Deciduous shrub/tree cover type. 


\section{Riparian Shrub}

This cover type was comprised of deciduous shrubs including both hydrophytic and facultative species (Figure 15). Typical shrub species included willow (Salix spp.), dogwood, rose, and chokecherry (Prunus virginiana). Clematis (Clematis ligusticifolia) vine was also present. This cover type is extremely limited and does not occur on all project sites.

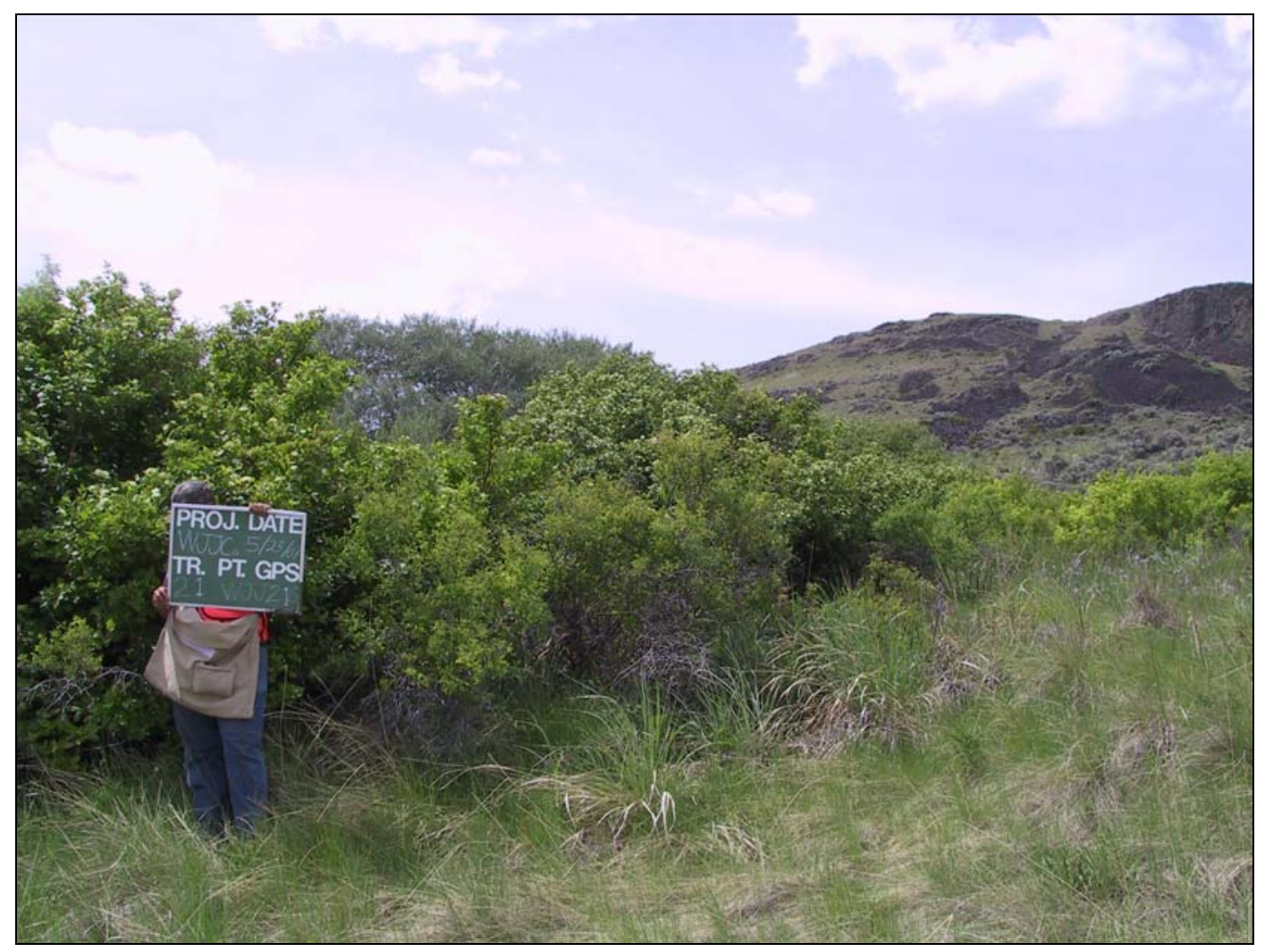

Figure 15. Riparian shrub cover type example.

\section{Riparian Forest}

The riparian forest ${ }^{7}$ cover type was dominated by quaking aspen trees (Figure 16). The shrub understory was comprised of rose, sapling aspen, choke cherry, and trace amounts of big sagebrush. Like riparian shrub, this cover type was limited in acreage and was evaluated only at the JoJaCo site.

\footnotetext{
${ }^{7}$ The riparian forest and deciduous shrub/tree cover types were very similar relative to woody plant composition. The primary distinction was that riparian forest had surface water present when HEP surveys were conducted. In addition, the deciduous shrub/tree cover type also included sites comprised of introduced poplar trees. These cover types could be combined with little, if any, impact to HEP model output.
} 


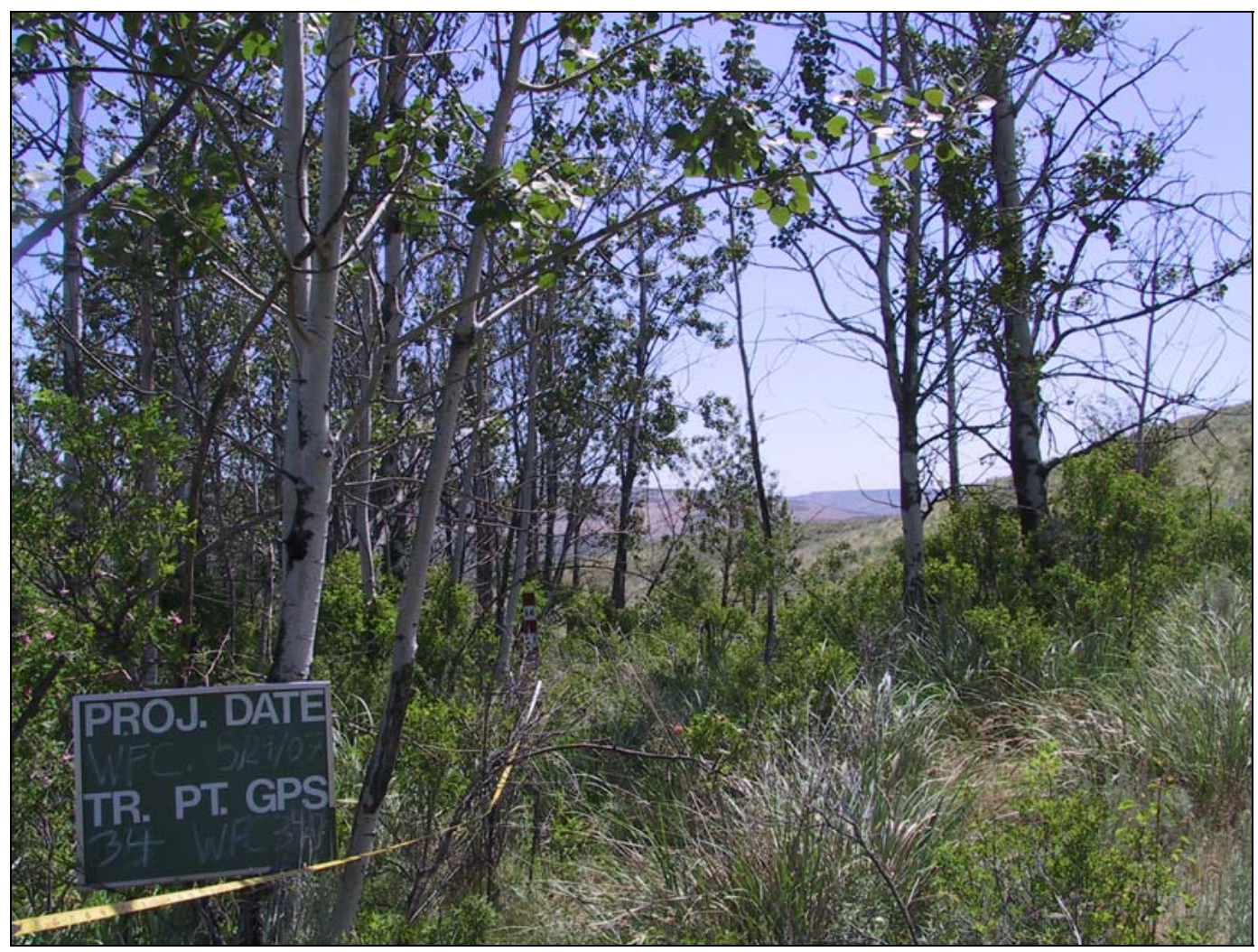

Figure 16. Riparian forest cover type example.

\section{Emergent Wetland}

Emergent wetlands generally support rush (Scirpus spp.) and limited amounts of cattail (Typhus latifolia). Carex species (Carex spp.) and juncos (Juncus spp.) may also be present. Most emergent wetlands were ephemeral (D. Peterson, pers. comm.). An example of an emergent wetland is illustrated in Figure 17. 


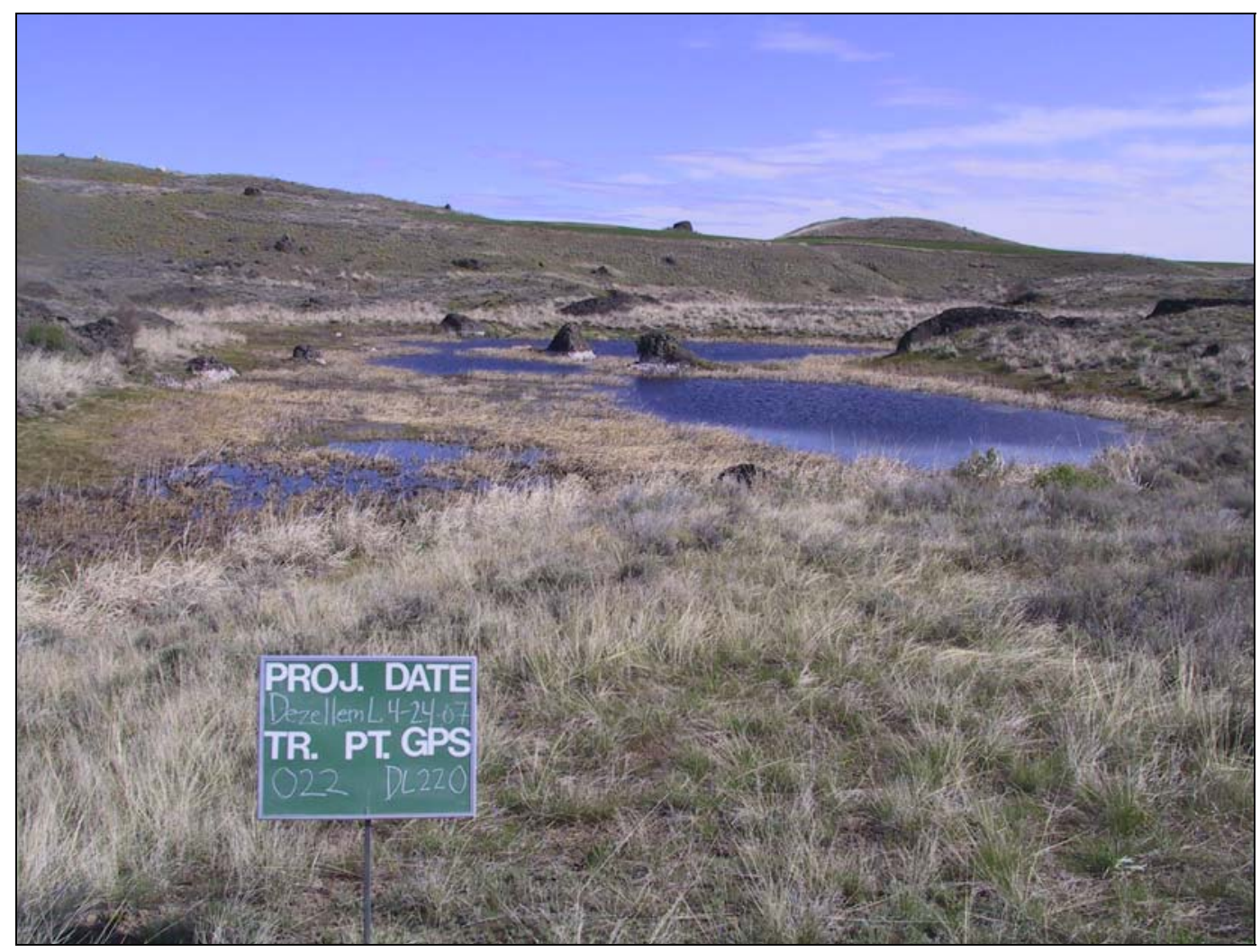

Figure 17. Emergent wetland example.

\section{Lacustrine}

The lacustrine cover type included perennial open water sites $\geq 2$ acres in size with less than $5 \%$ emergent vegetation. This cover type occurred on the McClain Lake and Dezellum Lake parcels. An example of the lacustrine cover type is shown in Figure 18. 


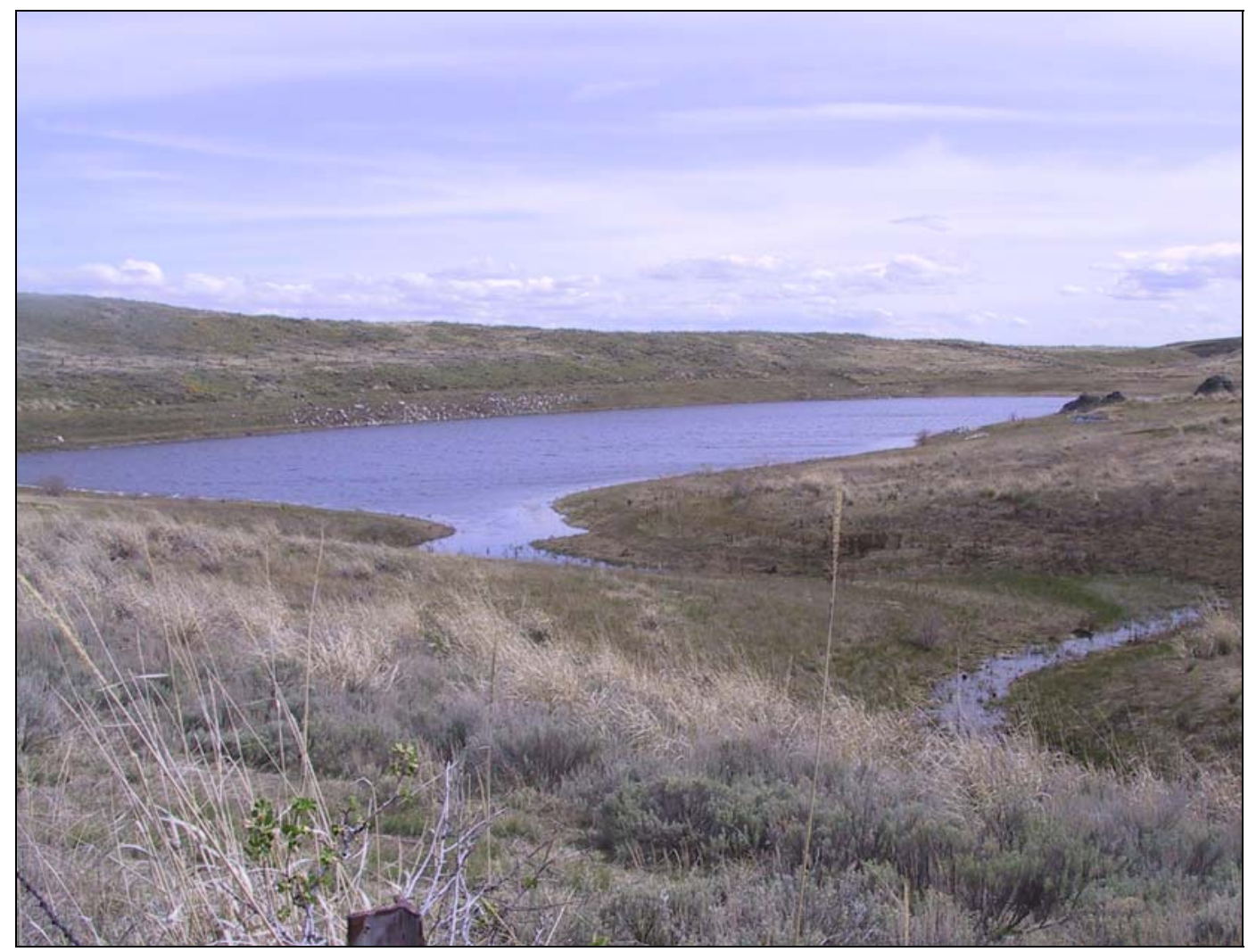

Figure 18. Lacustrine cover type example.

\section{Methods}

\section{Habitat Evaluation Procedures}

A habitat evaluation procedures analysis was conducted on West Foster Creek Expansion Project sites to document baseline habitat conditions and to determine how many protection habitat units to credit BPA for providing funds to manage the project sites as partial mitigation for habitat losses associated with construction of Grand Coulee and Chief Joseph Dams. HEP, developed by the U.S. Fish and Wildlife Service (USFWS), is used to quantify the impacts of development, protection, and restoration projects/measures on terrestrial and aquatic habitats by assessing changes, both negative and positive, in habitat quality and quantity (USFWS 1980, 1980a).

HEP is a habitat based approach to impact assessment that documents change through use of a habitat suitability index (HSI). The HSI value is derived from an evaluation of the ability of key habitat components to provide the life requisites of selected wildlife and fish species.

The HSI value is an index to habitat carrying capacity for a specific species or guild of species based on a performance measure (e.g. number of deer per square mile) described in HEP species models. The index ranges from 0.0 to 1.0. A HSI of 0.3 indicates that habitat quality/carrying capacity is marginal while a HSI of 0.7 suggests that habitat quality/carrying capacity is relatively good for a particular species (Table 3 ). 
Table 3. Habitat suitability index verbal equivalency table.

\begin{tabular}{cc}
\hline Habitat Suitability Index & Verbal Equivalent \\
\hline $0.0<0.2$ & Poor \\
$0.2<0.4$ & Marginal \\
$0.4<0.6$ & Fair \\
$0.6<0.9$ & Good \\
$0.9<1.0$ & Optimum \\
\hline
\end{tabular}

Each increment of change is identical. For example, a change in HSI from 0.1 to 0.2 represents the same magnitude of change as a change from 0.2 to 0.3 , and so forth. Habitat variables, suggested mensuration techniques, and mathematical aggregations of assessment results are included in HEP evaluation species models.

Habitat units are determined by multiplying the habitat suitability index by the number of acres of habitat (cover type) protected. For example, if the HSI output for a mule deer HEP model is 0.5 and the number of acres of shrubsteppe habitat protected is 100 , then the number of HUs are $50(0.5$ HSI x 100 acres $=50$ HUs $)$.

\section{HEP Model Selection}

HEP model selection was based on habitat types and species models identified in the Grand Coulee Dam (Howerton et al. 1986) and Chief Joseph Dam (Berger and Kuehn 1992) Loss Assessments. Howerton et al. (1986) did not clearly assign HEP species models to specific cover types making it difficult to develop a concise species/cover type matrix ${ }^{8}$. In addition, contrary to HEP protocols two "cover type" HEP models (riparian shrub and riparian forest) were also included in the loss assessment.

Specific HEP models were not included in the Grand Coulee Dam Loss Assessment (Howerton et al. 1986) and were unavailable for the West Foster Creek Expansion Project HEP assessment. Therefore, models from other sources were used to evaluate the West Foster Creek sites. In contrast, Berger and Kuehn (1992) included the bobcat HSI model in the Chief Joseph Dam Loss Assessment, which was used to evaluate the rock/cliff/talus cover type.

Consistent with other WDFW mitigation projects, HEP models selected by the Regional HEP Team to assess baseline habitat conditions included mule deer (Odocoileus hemionus) (Ashley and Berger 1996), western meadowlark (Sturnella neglecta) (Schroeder and Sousa 1982), sharptailed grouse (Ashley 2003), Bobcat (Lynx rufus) (Bodurtha 1991), mink (Neovison vison) (Allen 1984), mallard (Anas platyrhynchos) (WDFW 1999), and black-capped chickadee (Parus atricapillus) (Schroeder 1983). Abbreviated HEP models are included in Appendix A.

\footnotetext{
${ }^{8}$ The Coulee Dam species/cover type matrix is a draft document and subject to debate. It is, however, based on the best available data.

${ }^{9}$ By definition, cover types cannot be HEP models because HEP models must include a wildlife species.
} 
The 2007 West Foster Creek Expansion Project HEP evaluation cover type/species matrix shown in Table 4 was based primarily on information from the Grand Coulee Dam loss assessment (Howerton et al. 1986). The matrix also includes the rock/cliff/talus cover type and bobcat HSI model identified in the Chief Joseph Dam loss assessment (Berger and Kuehn 1992). As a result, bobcat habitat units were credited against Chief Joseph Dam while all other HU gains were credited against losses at Grand Coulee Dam.

Table 4. West Foster Creek Expansion Project cover type/HEP species model matrix.

\begin{tabular}{|c|c|c|c|}
\hline Parcel & Cover Type & Acres & HEP Model(s) \\
\hline \multirow[t]{2}{*}{ North Bridgeport } & Shrubsteppe ${ }^{1}$ & 314.10 & $\begin{array}{l}\text { Western Meadowlark, Mule } \\
\text { Deer, Sharp-tailed Grouse }\end{array}$ \\
\hline & Deciduous Shrub/Tree & 6.40 & Sharp-tailed Grouse \\
\hline Total & & 320.50 & \\
\hline Middle Bridgeport & Shrubsteppe ${ }^{1}$ & 161.90 & $\begin{array}{l}\text { Western Meadowlark, Mule } \\
\text { Deer, Sharp-tailed Grouse }\end{array}$ \\
\hline Total & & 161.90 & \\
\hline \multirow{5}{*}{ McClain Lake } & Shrubsteppe (shrubland) & 354.26 & $\begin{array}{l}\text { Western Meadowlark, Mule } \\
\text { Deer, Sharp-tailed Grouse }\end{array}$ \\
\hline & Grassland & 90.00 & $\begin{array}{l}\text { Western Meadowlark, Mule } \\
\text { Deer, Sharp-tailed Grouse }\end{array}$ \\
\hline & Deciduous Shrub/Tree & 14.50 & Sharp-tailed Grouse \\
\hline & Lacustrine & 9.76 & Mink \\
\hline & Emergent Wetland & 0.67 & Mink \\
\hline Total & & 469.19 & \\
\hline \multirow{3}{*}{ Dezellum Lake } & Shrubsteppe ${ }^{1}$ & 156.58 & $\begin{array}{l}\text { Western Meadowlark, Mule } \\
\text { Deer, Sharp-tailed Grouse }\end{array}$ \\
\hline & Emergent Wetland & 5.00 & Mallard \\
\hline & Lacustrine & 5.00 & Mallard \\
\hline Total & & 166.58 & \\
\hline \multirow{5}{*}{ JoJaCo } & Shrubsteppe ${ }^{1}$ & $2,593.31$ & $\begin{array}{l}\text { Western Meadowlark, Mule } \\
\text { Deer, Sharp-tailed Grouse }\end{array}$ \\
\hline & Riparian Shrub & 8.00 & Sharp-tailed Grouse \\
\hline & Riparian Forest & 22.00 & Sharp-tailed Grouse \\
\hline & Conifer Woodland & 7.00 & Black-capped Chickadee \\
\hline & Rock/Cliff/Talus & 8.00 & Bobcat \\
\hline Total & & $2,638.31$ & \\
\hline
\end{tabular}

\section{HEP Species Model Selection Rationale}

Species selection rationale described in the Grand Coulee Dam Loss Assessment (Howerton et al. 1986) and from the Chief Joseph loss assessment (Berger and Kuehn 1992) is summarized in Table 5. 
Table 5. HEP model species selection rationale table.

\begin{tabular}{|l|l|}
\hline \multicolumn{1}{|c|}{ HEP Model } & \multicolumn{1}{c|}{ Rationale } \\
\hline Mule deer & $\begin{array}{l}\text { This species represents wildlife dependent upon shrubsteppe and river } \\
\text { breaks. }\end{array}$ \\
\hline Western meadowlark & $\begin{array}{l}\text { Represents wildlife species dependent upon grassland and/or } \\
\text { shrubsteppe habitats. }\end{array}$ \\
\hline Sharp-tailed grouse & $\begin{array}{l}\text { Represents wildlife species dependent upon grasslands/shrubsteppe } \\
\text { habitat (includes riparian draws and limited agriculture). }\end{array}$ \\
\hline Bobcat & $\begin{array}{l}\text { Represents wildlife species dependent upon rocky areas and adjacent } \\
\text { grassland/shrubsteppe habitat. } \\
\text { Bhe species represents wildlife dependent upon forest habitats and } \\
\text { chickadee }\end{array}$ \\
\hline Mink & $\begin{array}{l}\text { Represents wildlife species dependent upon open water areas and } \\
\text { adjacent cover that are sensitive to shoreline development. }\end{array}$ \\
\hline Mallard & Represents waterfowl brood rearing habitat. \\
\hline
\end{tabular}

\section{Sampling Design and Measurement Protocols}

\section{Meta Data}

Level one meta data follows that suggested by Gotelli and Ellison (2004). Field surveys were conducted by the Columbia Basin Fish and Wildlife Authority's Regional HEP Team with assistance from WDFW Wildlife Area Assistant Dan Peterson. Regional HEP Team members included Paul Ashley (RHT Coordinator), Mike Cantonese (Team Leader), Anthony Muse, Paul Walker, and Tiffany Baker (contact Paul Ashley@ 1onepinebutte@comcast.net or through CBFWA at: [503] 229-0191).

Funding for the HEP analyses was provided by Bonneville Power Administration with RHT administrative support provided by CBFWA. Specific measurement techniques and protocols are described in detail in Appendix B. Measurements were recorded in standard English units except the Robel pole (Robel et al. 1975), which was recorded in metric units i.e., decimeters.

\section{Transect Methods}

In most cases, the Regional HEP team used measurement techniques and protocols described in HEP models to evaluate habitat variables; however, ocular estimations were used when direct measurements could not be taken. Measured techniques were occasionally modified to meet unique habitat and/or physiographic conditions. Metrics generally followed those described by Hays et al. (1981) and/or Avery (1994).

Stratified (by cover type), random transects were established and documented using global positioning system (GPS) coordinates and, in many cases, rebar stakes. Ashley (2006) described the methods and protocols used by Regional HEP Team staff to collect HEP model variable data and additional floristic information (Appendix B). Field data was summarized and applied to HEP model variables to determine habitat suitability indices and habitat units for each HEP 
species model. Field data collection and processing procedures are illustrated in Figure 19 and summarized as follows.

HEP model variable field data was entered onto Allegro CE® data logger spreadsheets (1), or recorded on paper data sheets (2). The raw field data (3) was downloaded from the data loggers or manually entered from paper data sheets onto computers (transect photos were also downloaded and stored on field computers). The raw data and photos were compiled for each transect into three basic products/files (4) that are provided to project managers as report appendices and/or separate CD files.

Product files included raw field data downloaded from the data loggers (5), data summary spreadsheets (6) which are the results of compiling/processing the raw data, and transect photo files (7). Summarized/processed data from each transect was applied to appropriate HEP model variables to determine suitability index (SI) ratings that were combined on habitat suitability index (HSI) spreadsheets (8) to determine the HSI for a particular HEP species model/cover type. The habitat suitability index was then multiplied by the number of cover type acres to determine the number of habitat units (9). 


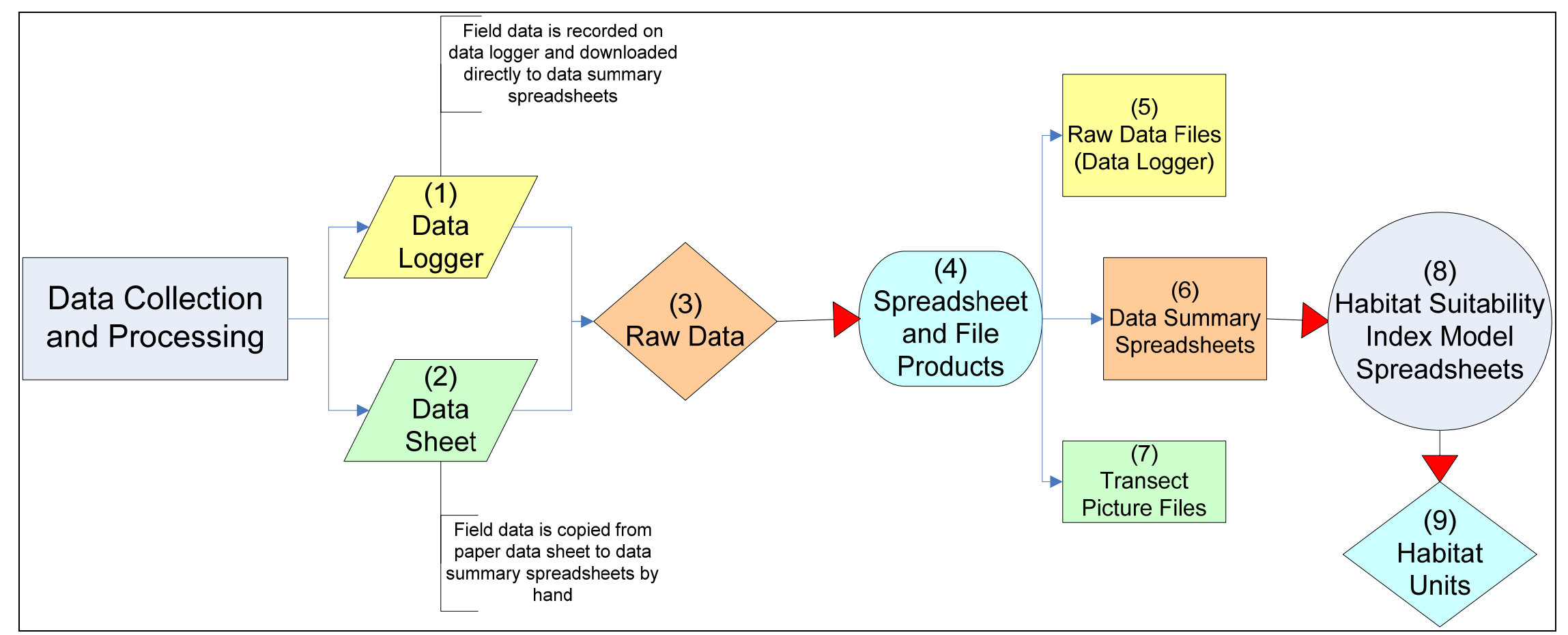

Figure 19. HEP data collection and processing flow chart. 


\section{Transect Locations}

Transect initial points (IPs) were established based on stratified random sampling protocols with cover types defining the strata. The number of samples initially allocated per cover type strata were determined based on a proportional allocation strategy (Husch et al. 2003). Specific IP locations were identified by electronically superimposing either a $50 \mathrm{~m} \times 50 \mathrm{~m}$ or $100 \mathrm{~m} \times 100 \mathrm{~m}$ grid over cover types and selecting random numbers to identify "XY" point coordinates. IPs were occasionally located off the grid to accommodate cover types comprised of few acres or those of special interest (P. Ashley, pers. comm.).

The proportional allocation strategy was modified in the field as needed to compensate for the relative homogeneity of a particular cover type; to account for unanticipated access issues and/or physiographic restrictions; and/or to meet temporal considerations. In addition, initial points were moved when they did not fall within the cover type(s) of interest, or were in inaccessible areas such as the middle of a pond or cliff area (additional transect information is located in Appendix B).

Transect UTM coordinates (NAD 27) for start, turn, and end points were recorded in the field on a Garmin IIIA ${ }^{\circledR}$ GPS unit. Site transect location maps are included in Appendix C (aerial photographs). IP/transect UTM coordinates, transect magnetic azimuths, and transect lengths are summarized in Table 6.

Table 6. West Foster Creek Expansion project transect coordinates, azimuths, and lengths.

\begin{tabular}{|c|c|c|c|c|c|c|}
\hline \multirow{3}{*}{$\begin{array}{c}\begin{array}{c}\text { Project \& Transect } \\
\text { No. }\end{array} \\
\text { North Bridgeport }\end{array}$} & \multirow{3}{*}{ Point } & \multirow{2}{*}{\multicolumn{2}{|c|}{$\begin{array}{c}\text { GPS } \\
\text { UTM Coordinates }\end{array}$}} & \multirow{3}{*}{$\begin{array}{l}\text { Magnetic } \\
\text { Azimuth } \\
\text { (Degrees) }\end{array}$} & \multirow{3}{*}{$\begin{array}{l}\text { Length } \\
\text { (Feet) }\end{array}$} & \multirow{2}{*}{$\begin{array}{l}\text { Total } \\
\text { Length }\end{array}$} \\
\hline & & & & & & \\
\hline & & E & $\mathbf{N}$ & & & \\
\hline \multirow[t]{2}{*}{ Tran. 01} & start & 0298596 & 5323600 & 127 & $300^{\prime}$ & \\
\hline & end & 0298581 & 5323515 & - & - & $300^{\prime}$ \\
\hline \multirow[t]{2}{*}{ Tran. 02} & start & 0298448 & 5323591 & - & - & - \\
\hline & end & - & - & - & - & Ocular \\
\hline \multirow[t]{2}{*}{ Tran. 04} & start & 0298691 & 5323502 & 005 & $300^{\prime}$ & - \\
\hline & end & 0298730 & 5323585 & - & - & 300 \\
\hline \multirow[t]{3}{*}{ Tran. 05} & start & 0298697 & 5323299 & 284 & $200^{\prime}$ & - \\
\hline & turn & 0298651 & 5323340 & 239 & $100^{\prime}$ & \\
\hline & end & 0298621 & 5323331 & - & - & $300^{\prime}$ \\
\hline \multirow[t]{2}{*}{ Tran. 06} & start & 0298600 & 5323406 & GreenLine & $300^{\prime}$ & - \\
\hline & end & \multicolumn{3}{|c|}{ Coordinates not available } & - & 300 \\
\hline \multirow[t]{2}{*}{ Tran. 16} & start & 0298646 & 5323005 & 340 & 300 & - \\
\hline & end & 0298638 & 5323099 & - & - & 300 \\
\hline \multirow[t]{2}{*}{ Tran. 18} & start & 0298506 & 5322805 & 331 & 300 & - \\
\hline & end & 0298473 & 5322885 & - & - & 300 \\
\hline \multirow[t]{2}{*}{ Tran. 19} & start & 0298297 & 5322707 & 050 & 300 & - \\
\hline & end & 0298376 & 5322752 & - & - & 300 \\
\hline Tran. 20 & start & 0298498 & 5322526 & 280 & $300^{\prime}$ & - \\
\hline
\end{tabular}


West Foster Creek Expansion Project

\begin{tabular}{|c|c|c|c|c|c|c|}
\hline \multirow{3}{*}{$\begin{array}{c}\text { Project \& Transect } \\
\text { No. }\end{array}$} & \multirow{3}{*}{$\begin{array}{r}\text { Point } \\
\text { end } \\
\end{array}$} & \multirow{2}{*}{\multicolumn{2}{|c|}{$\begin{array}{c}\text { GPS } \\
\text { UTM Coordinates }\end{array}$}} & \multirow{3}{*}{$\begin{array}{c}\text { Magnetic } \\
\text { Azimuth } \\
\text { (Degrees) } \\
-\end{array}$} & \multirow{3}{*}{$\begin{array}{c}\begin{array}{c}\text { Length } \\
\text { (Feet) }\end{array} \\
-\end{array}$} & \multirow{3}{*}{$\begin{array}{c}\begin{array}{c}\text { Total } \\
\text { Length }\end{array} \\
300^{\prime}\end{array}$} \\
\hline & & & & & & \\
\hline & & 0298439 & 5322574 & & & \\
\hline \multirow[t]{2}{*}{ Tran. 23} & start & 0298642 & 5322268 & 320 & $300^{\prime}$ & - \\
\hline & end & 0298602 & 5322362 & - & - & $300^{\prime}$ \\
\hline \multirow[t]{3}{*}{ Tran. 24} & start & 0298822 & 5322098 & 070 & $100^{\prime}$ & - \\
\hline & turn & 0298826 & 5322107 & 025 & $200^{\prime}$ & - \\
\hline & end & 0298870 & 5322160 & - & - & $300^{\prime}$ \\
\hline \multirow[t]{2}{*}{ Tran. 25} & start & 0298810 & 5321880 & GreenLine & $300^{\prime}$ & - \\
\hline & end & 0298851 & 5321961 & - & - & $300^{\prime}$ \\
\hline \multirow[t]{2}{*}{ Tran. 26} & start & 0298898 & 5321799 & 030 & $300^{\prime}$ & - \\
\hline & end & 0298968 & 5321859 & - & - & $300^{\prime}$ \\
\hline \multicolumn{7}{|l|}{ McClain Lake } \\
\hline \multirow[t]{2}{*}{ Tran. 41} & start & 0295658 & 5319215 & 90 & $300^{\prime}$ & - \\
\hline & end & 0295750 & 5319198 & - & - & $300^{\prime}$ \\
\hline \multirow[t]{2}{*}{ Tran. 42} & start & 0296010 & 5319204 & 119 & $300^{\prime}$ & - \\
\hline & end & 0296067 & 5319141 & - & - & $300^{\prime}$ \\
\hline \multirow[t]{2}{*}{ Tran. 43} & start & 0296170 & 5319025 & & & \\
\hline & end & - & - & & & Ocular \\
\hline \multirow[t]{2}{*}{ Tran. 45} & start & 0295496 & 5318907 & 360 & $300^{\prime}$ & - \\
\hline & end & 0295521 & 5318993 & - & - & $300^{\prime}$ \\
\hline \multirow[t]{2}{*}{ Tran. 46} & start & 0296000 & 5318750 & 206 & $300^{\prime}$ & - \\
\hline & end & 0295945 & 5318691 & - & - & $300^{\prime}$ \\
\hline \multirow[t]{2}{*}{ Tran. 47} & start & 0295703 & 5318714 & 330 & $300^{\prime}$ & - \\
\hline & end & 0295688 & 5318805 & - & - & $300^{\prime}$ \\
\hline \multirow[t]{2}{*}{ Tran. 49} & start & 0296590 & 5318438 & 224 & $300^{\prime}$ & - \\
\hline & end & 0296531 & 5318439 & - & - & $300^{\prime}$ \\
\hline \multirow[t]{3}{*}{ Tran. 51} & start & 0296495 & 5318170 & 180 & $300^{\prime}$ & - \\
\hline & turn & 0296480 & 5318112 & & & \\
\hline & end & 0296479 & 5318113 & - & - & 300 \\
\hline \multirow[t]{2}{*}{ Tran. 52} & start & 0296348 & 5318037 & 183 & $300^{\prime}$ & - \\
\hline & end & 0296384 & 5317943 & - & - & $300^{\prime}$ \\
\hline \multirow[t]{2}{*}{ Tran. 57} & start & 0296501 & 5317339 & 074 & $300^{\prime}$ & - \\
\hline & end & 0296635 & 5317327 & - & - & $300^{\prime}$ \\
\hline \multirow[t]{2}{*}{ Tran. 59} & start & 0296495 & 5317111 & 077 & $300^{\prime}$ & - \\
\hline & end & 0296617 & 5317101 & - & - & $300^{\prime}$ \\
\hline \multirow[t]{2}{*}{ Tran. 70} & start & 0296339 & 5317889 & GreenLine & $300^{\prime}$ & - \\
\hline & end & - & - & - & - & 300 \\
\hline \multirow[t]{2}{*}{ Tran. 71} & start & 0296459 & 5317808 & 120 & $300^{\prime}$ & - \\
\hline & end & 0296506 & 5317730 & - & - & $300^{\prime}$ \\
\hline Tran. 72 & start & 0296643 & 5316983 & - & - & - \\
\hline & end & - & - & - & - & Ocular \\
\hline Dezellem Lake & & & & & & \\
\hline Tran. 02 & start & 0293876 & 5318405 & 110 & $300^{\prime}$ & - \\
\hline
\end{tabular}


West Foster Creek Expansion Project

\begin{tabular}{|c|c|c|c|c|c|c|}
\hline \multirow{3}{*}{$\begin{array}{c}\text { Project \& Transect } \\
\text { No. }\end{array}$} & \multirow{3}{*}{$\begin{array}{r}\text { Point } \\
\text { end } \\
\end{array}$} & \multirow{2}{*}{\multicolumn{2}{|c|}{$\begin{array}{c}\text { GPS } \\
\text { UTM Coordinates }\end{array}$}} & \multirow{3}{*}{$\begin{array}{c}\text { Magnetic } \\
\text { Azimuth } \\
\text { (Degrees) } \\
-\end{array}$} & \multirow{3}{*}{$\begin{array}{c}\text { Length } \\
\text { (Feet) } \\
-\end{array}$} & \multirow{3}{*}{$\begin{array}{c}\begin{array}{c}\text { Total } \\
\text { Length }\end{array} \\
300^{\prime}\end{array}$} \\
\hline & & & & & & \\
\hline & & 0293968 & 5318347 & & & \\
\hline \multirow[t]{2}{*}{ Tran. 03} & start & 0293920 & 5318589 & 247 & $300^{\prime}$ & - \\
\hline & end & 0293831 & 5318579 & - & - & $300^{\prime}$ \\
\hline \multirow[t]{2}{*}{ Tran. 04} & start & 0294102 & 5318396 & 283 & $300^{\prime}$ & - \\
\hline & end & 0294018 & 5318454 & - & - & $300^{\prime}$ \\
\hline \multirow[t]{2}{*}{ Tran. 06} & start & 0294300 & 5318398 & 186 & $300^{\prime}$ & - \\
\hline & end & 0294266 & 5318319 & - & - & $300^{\prime}$ \\
\hline \multirow[t]{2}{*}{ Tran. 07} & start & 0294401 & 5318605 & 219 & $300^{\prime}$ & - \\
\hline & end & 0294314 & 5318561 & - & - & $300^{\prime}$ \\
\hline \multirow[t]{2}{*}{ Tran. 10} & start & 0294609 & 5318672 & 138 & $300^{\prime}$ & - \\
\hline & end & 0294656 & 5318585 & - & - & $300^{\prime}$ \\
\hline \multirow[t]{2}{*}{ Tran. 13} & start & 0294007 & 5318205 & 070 & $300^{\prime}$ & - \\
\hline & End & 0294092 & 5318211 & - & - & $300^{\prime}$ \\
\hline \multirow[t]{2}{*}{ Tran. 14} & start & 0294216 & 5318176 & - & OC & - \\
\hline & end & - & - & - & - & Ocular \\
\hline \multirow[t]{2}{*}{ Tran. 17} & start & 0294399 & 5317906 & 009 & $300^{\prime}$ & - \\
\hline & end & 0294435 & 5317989 & - & - & $300^{\prime}$ \\
\hline \multirow[t]{2}{*}{ Tran. 22} & start & 0294098 & 5318449 & - & OC & - \\
\hline & end & - & - & - & - & Ocular \\
\hline \multirow[t]{2}{*}{ Tran. 23} & start & 0294084 & 5318256 & - & OC & - \\
\hline & end & - & - & - & - & Ocular \\
\hline \multirow[t]{2}{*}{ Tran. 24} & start & 0294478 & 5318222 & - & OC & - \\
\hline & end & - & - & - & - & Ocular \\
\hline \multirow[t]{2}{*}{ Tran. 25} & start & 0294606 & 5318566 & - & $\mathrm{OC}$ & - \\
\hline & end & - & - & - & - & Ocular \\
\hline \multicolumn{7}{|l|}{ Middle Bridgeport } \\
\hline \multirow[t]{2}{*}{ Tran. 32} & start & 0297404 & 5320406 & 090 & $300^{\prime}$ & - \\
\hline & end & 0297481 & 5320372 & - & - & $300^{\prime}$ \\
\hline \multirow[t]{2}{*}{ Tran. 34} & start & 0297617 & 5320223 & 010 & $300^{\prime}$ & - \\
\hline & end & 0297661 & 5320331 & - & - & $300^{\prime}$ \\
\hline \multirow[t]{2}{*}{ Tran. 35} & start & 0297276 & 5320274 & 326 & $300^{\prime}$ & - \\
\hline & end & 0297245 & 5320361 & - & - & $300^{\prime}$ \\
\hline \multirow[t]{3}{*}{ Tran. 36} & start & 0297136 & 5320017 & 348 & 300 & - \\
\hline & turn & 0297170 & 5320085 & 087 & $100^{\prime}$ & \\
\hline & end & 0297190 & 5320088 & - & - & 400 \\
\hline \multirow[t]{2}{*}{ Tran. 37} & start & 0297290 & 5319857 & 357 & $300^{\prime}$ & - \\
\hline & end & 0297304 & 5319944 & - & - & $300^{\prime}$ \\
\hline Tran. 39 & start & 0297302 & 5319513 & 334 & $300^{\prime}$ & - \\
\hline & end & 0297280 & 5319603 & - & - & 300 \\
\hline Tran. 40 & start & 0297098 & 5319416 & 360 & $300^{\prime}$ & - \\
\hline & end & 0297127 & 5319504 & - & - & 300 \\
\hline JoJaCo. & & & & & & \\
\hline
\end{tabular}


West Foster Creek Expansion Project

\begin{tabular}{|c|c|c|c|c|c|c|}
\hline \multirow{3}{*}{$\begin{array}{c}\text { Project \& Transect } \\
\text { No. } \\
\text { Tran. } 20 \\
\end{array}$} & \multirow{3}{*}{$\begin{array}{r}\text { Point } \\
\text { start }\end{array}$} & \multirow{2}{*}{\multicolumn{2}{|c|}{$\begin{array}{c}\text { GPS } \\
\text { UTM Coordinates }\end{array}$}} & \multirow{3}{*}{$\begin{array}{l}\text { Magnetic } \\
\text { Azimuth } \\
\text { (Degrees) }\end{array}$} & \multirow{3}{*}{$\begin{array}{c}\begin{array}{c}\text { Length } \\
\text { (Feet) }\end{array} \\
300 \\
\end{array}$} & \multirow{3}{*}{$\begin{array}{l}\text { Total } \\
\text { Length }\end{array}$} \\
\hline & & & & & & \\
\hline & & 0300423 & 5315750 & & & \\
\hline & end & 0300478 & 5315816 & - & - & $300^{\prime}$ \\
\hline \multirow[t]{2}{*}{ Tran. 21} & start & 0300426 & 5315846 & - & - & - \\
\hline & end & - & - & - & - & - \\
\hline \multirow[t]{2}{*}{ Tran. 22} & start & 0300278 & 5315771 & 098 & $300^{\prime}$ & - \\
\hline & end & 0300367 & 5315767 & - & - & $300^{\prime}$ \\
\hline \multirow[t]{2}{*}{ Tran. 23} & start & 0300243 & 5315634 & 280 & $300^{\prime}$ & - \\
\hline & end & 0300164 & 5315683 & - & - & $300^{\prime}$ \\
\hline \multirow[t]{2}{*}{ Tran. 24} & start & 0300225 & 5315120 & 175 & $300^{\prime}$ & - \\
\hline & end & 0300217 & 5315041 & - & - & $300^{\prime}$ \\
\hline \multirow[t]{2}{*}{ Tran. 25} & start & 0301431 & 5316921 & 083 & $150^{\prime}$ & - \\
\hline & end & 0301467 & 5316911 & - & - & 150' \\
\hline \multirow[t]{2}{*}{ Tran. 26} & start & 0301397 & 5316846 & 246 & $600^{\prime}$ & - \\
\hline & end & 0301219 & 5316840 & - & - & $600^{\prime}$ \\
\hline \multirow[t]{2}{*}{ Tran. 27} & start & 0298934 & 5319109 & Greenline & $300^{\prime}$ & - \\
\hline & end & 0298983 & 5319022 & - & - & $300^{\prime}$ \\
\hline \multirow[t]{2}{*}{ Tran. 28} & start & 0298935 & 5318953 & 154 & $300^{\prime}$ & - \\
\hline & end & 0298952 & 5318863 & - & - & $300^{\prime}$ \\
\hline \multirow[t]{2}{*}{ Tran. 29} & start & 0299037 & 5318825 & 151 & $300^{\prime}$ & - \\
\hline & end & 0299064 & 5318736 & - & - & $300^{\prime}$ \\
\hline \multirow[t]{2}{*}{ Tran. 30} & start & 0301636 & 5317490 & 118 & $600^{\prime}$ & - \\
\hline & end & 0301757 & 5317363 & - & - & $600^{\prime}$ \\
\hline \multirow[t]{2}{*}{ Tran. 31} & start & 0300499 & 5317432 & 328 & $300^{\prime}$ & - \\
\hline & end & 0300490 & 5317520 & - & - & $300^{\prime}$ \\
\hline \multirow[t]{2}{*}{ Tran. 32} & start & 0300308 & 5317372 & 222 & $300^{\prime}$ & - \\
\hline & end & 0300238 & 5317333 & - & - & $300^{\prime}$ \\
\hline \multirow[t]{2}{*}{ Tran. 33} & start & 0300020 & 5317620 & 318 & $300^{\prime}$ & - \\
\hline & end & 0299986 & 5317689 & - & - & $300^{\prime}$ \\
\hline \multirow[t]{2}{*}{ Tran. 34} & start & 0299928 & 5317579 & 091 & $300^{\prime}$ & - \\
\hline & end & 0300022 & 5317563 & - & - & $300^{\prime}$ \\
\hline \multirow[t]{2}{*}{ Tran. 35} & start & 0300235 & 5317733 & 080 & $300^{\prime}$ & - \\
\hline & end & 0300277 & 5317815 & - & - & $300^{\prime}$ \\
\hline \multirow[t]{3}{*}{ Tran. 36} & start & 0299855 & 5318122 & 300 & $200^{\prime}$ & - \\
\hline & turn & 0299818 & 5318132 & 265 & $100^{\prime}$ & - \\
\hline & end & 0299796 & 5318142 & - & $300^{\prime}$ & $600^{\prime}$ \\
\hline \multirow[t]{2}{*}{ Tran. 37} & start & 0299578 & 5318556 & 324 & $300^{\prime}$ & - \\
\hline & end & 0299542 & 5318648 & - & - & $300^{\prime}$ \\
\hline \multirow[t]{2}{*}{ Tran. 38} & start & 0299504 & 5318540 & unknown & $300^{\prime}$ & - \\
\hline & end & - & - & - & - & $300^{\prime}$ \\
\hline Tran. 39 & start & 0298589 & 5319802 & Greenline & - & - \\
\hline & end & 0298651 & 5319857 & - & - & - \\
\hline Tran. 40 & start & 0298695 & 5319944 & 120 & $300^{\prime}$ & - \\
\hline
\end{tabular}


West Foster Creek Expansion Project

\begin{tabular}{|c|c|c|c|c|c|c|}
\hline \multirow{3}{*}{$\begin{array}{c}\text { Project \& Transect } \\
\text { No. }\end{array}$} & \multirow{3}{*}{$\begin{array}{r}\text { Point } \\
\text { end } \\
\end{array}$} & \multirow{2}{*}{\multicolumn{2}{|c|}{$\begin{array}{c}\text { GPS } \\
\text { UTM Coordinates }\end{array}$}} & \multirow{3}{*}{$\begin{array}{c}\text { Magnetic } \\
\text { Azimuth } \\
\text { (Degrees) } \\
-\end{array}$} & \multirow{3}{*}{$\begin{array}{c}\text { Length } \\
\text { (Feet) }\end{array}$} & \multirow{3}{*}{$\begin{array}{c}\begin{array}{c}\text { Total } \\
\text { Length }\end{array} \\
300^{\prime}\end{array}$} \\
\hline & & & & & & \\
\hline & & 0298751 & 5319875 & & & \\
\hline \multirow[t]{2}{*}{ Tran. 41} & start & 0298755 & 5319717 & 185 & $300^{\prime}$ & - \\
\hline & end & 0298719 & 5319639 & - & - & 300 \\
\hline \multirow[t]{2}{*}{ Tran. 42} & start & 0298973 & 5320086 & 083 & 300 & - \\
\hline & end & 0299064 & 5320067 & - & - & 300 \\
\hline \multirow[t]{2}{*}{ Tran. 50} & start & 0299871 & 5319224 & 102 & 600 & - \\
\hline & end & 0300034 & 5319122 & - & - & 600 \\
\hline \multirow[t]{2}{*}{ Tran. 51} & start & 0299735 & 5319115 & Greenline & $300^{\prime}$ & - \\
\hline & end & 0299690 & 5319045 & - & - & $300^{\prime}$ \\
\hline \multirow[t]{2}{*}{ Tran. 52} & start & 0299602 & 5319482 & 315 & 600 & - \\
\hline & end & 0299514 & 5319667 & - & - & $600^{\prime}$ \\
\hline \multirow[t]{2}{*}{ Tran. 53} & start & 0300472 & 5318907 & 133 & $600^{\prime}$ & - \\
\hline & end & 0300568 & 5318750 & - & - & $600^{\prime}$ \\
\hline \multirow[t]{2}{*}{ Tran. 54} & start & 0299194 & 5316929 & 358 & 300 & - \\
\hline & end & 0299202 & 5317023 & - & - & $300^{\prime}$ \\
\hline \multirow[t]{2}{*}{ Tran. 55} & start & 0299054 & 5317083 & 267 & 300 & - \\
\hline & end & 0298944 & 5317099 & - & - & $300^{\prime}$ \\
\hline
\end{tabular}

\section{Transect Photo Documentation}

Transects were photographed with a Canon G1® 3.3 mega pixal digital camera (with and without magnification). Transect photographs are included in Appendix D.

\section{Photo Methods}

Photo points were established at the start point of each transect to document extant habitat conditions. Digital photographs were recorded from a height of three feet $(\approx 1$ meter) at the beginning of each transect facing the same direction as the transect azimuth.

A transect reference board ${ }^{10}$ was placed at the 15 foot interval while a cover board, divided into 3 inch $\times 4$ inch $(8 \mathrm{~cm} \times 10 \mathrm{~cm})$ rectangles, was set at the 30 foot mark on each transect. Panoramic photographs were recorded to document dense vegetation, linear/narrow cover types, and other "atypical" or "special interest" habitat conditions. An example of a photo documentation point is illustrated in Figure 20.

\footnotetext{
${ }^{10}$ Included transect number, project name, date, GPS reference number
} 


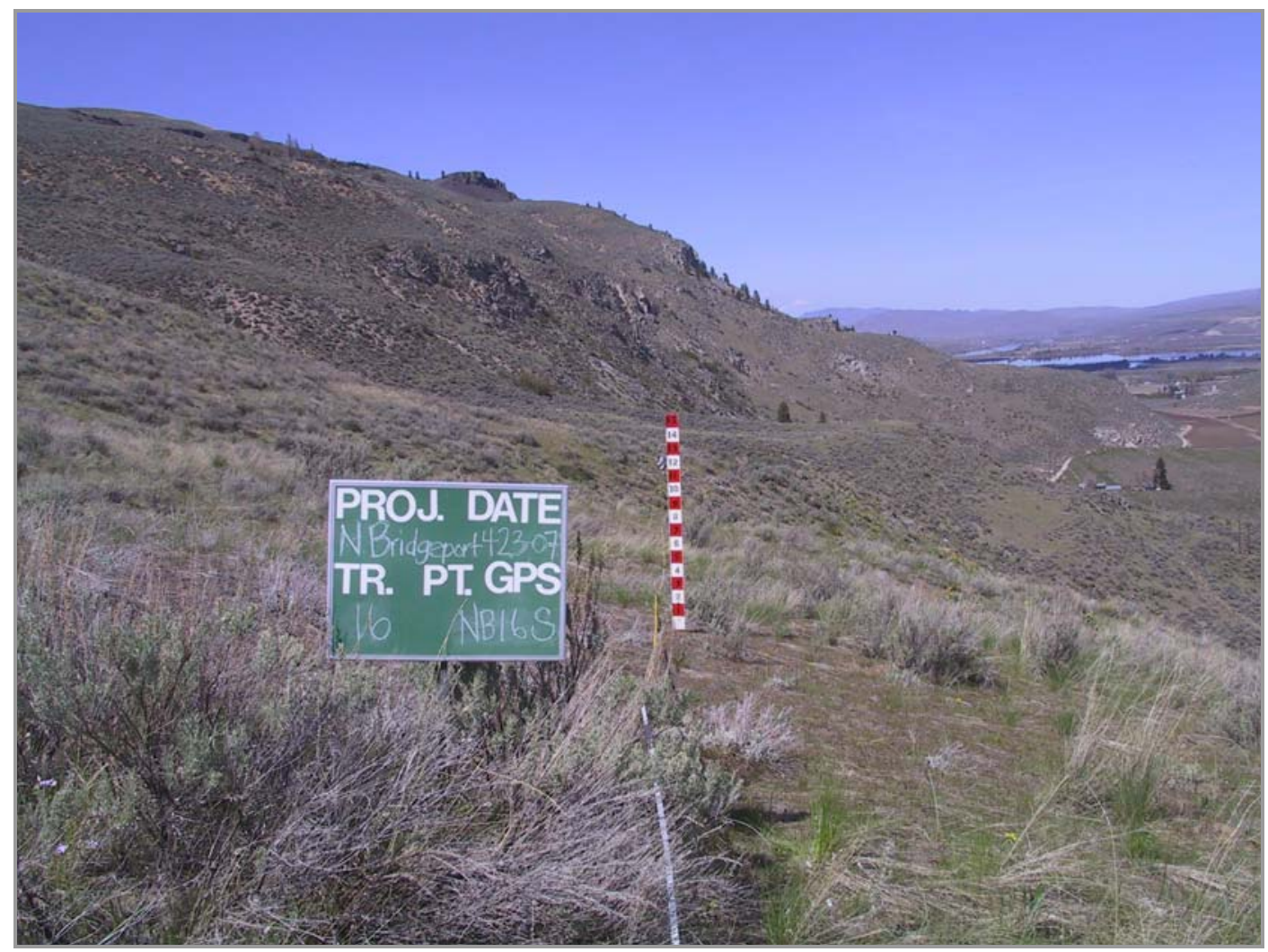

Figure 20. Transect photo point example.

\section{Results}

A Habitat Evaluation Procedures evaluation was conducted on West Foster Creek Expansion sites to assess habitat quality and to determine the number of baseline/protection habitat units (HUs) to credit BPA as partial mitigation for habitat losses associated with Grand Coulee and Chief Joseph Dams. Baseline HEP surveys conducted in April and May 2007 on the five WFCEP sites generated 4,946.44 habitat units or 1.32 HUs per acre.

HEP survey results are summarized by cover types and species for each site in Table 7 through Table 11. HEP species models and habitat suitability mathematical aggregations are included in Appendix A. Raw transect data and summary spreadsheets for all project areas can be viewed via the following links:
1. North Bridgeport
1. Middle Bridgeport
2. McClain Lake
3. Dezellum Lake
4. $\underline{\mathrm{JoJaCo}}$ 
West Foster Creek Expansion Project

\begin{tabular}{|c|c|c|c|c|c|c|c|}
\hline Cover Type & Acres & Model/Comments & Variable & SI & HSI & HUs & Remarks \\
\hline \multirow{23}{*}{ Shrubsteppe/Grassland } & \multirow{5}{*}{314.10} & \multirow{5}{*}{ W. Meadowlark } & V1: \% C.C. Herb. Plants & 0.79 & 0.16 & 50.13 & \\
\hline & & & V2: \% Herb. C.C. Composed of Grass & 1.00 & & & \\
\hline & & & V3: Ave. Ht. of Herb. Canopy & 0.59 & & & \\
\hline & & & V4: Distance to Perch Sites & 1.00 & & & \\
\hline & & & V5: \% Shrub Canopy Cover & 0.23 & & & \\
\hline & & & & & & & \\
\hline & & \multirow{5}{*}{ Mule Deer } & $\begin{array}{l}\text { V1: Percent cover of preferred } \\
\text { shrubs }<1.5 \text { meters in height }\end{array}$ & 0.85 & 0.43 & 136.25 & Food Index \\
\hline & & & V2: Percent cover of all shrubs $<1.5$ meters in height. & 0.85 & 0.80 & & Cover Index \\
\hline & & & V3: Mean shrub height. & 0.56 & & & \\
\hline & & & V4: Number of preferred shrub species. & 0.72 & & & \\
\hline & & & V5: Percent cover of palatable herbaceous species. & 1.00 & & & \\
\hline & & \multirow{5}{*}{ Landscape variables } & $\begin{array}{l}\text { V6: Presence of suitable agricultural crops } \\
\text { within } 1.6 \text { kilometers ( } 1 \text { mile) of study area }\end{array}$ & 0.10 & & & \\
\hline & & & V7: Aspect & 0.35 & & & \\
\hline & & & V8: Road density & 1.00 & & & \\
\hline & & & V9: Topographic diversity & 1.00 & & & \\
\hline & & & $\begin{array}{l}\text { V10: Percent evergreen canopy } \\
>1.5 \text { meters in height }\end{array}$ & 0.00 & & & \\
\hline & & \multirow{5}{*}{$\begin{array}{l}\text { Sharp-tailed Grouse } \\
\text { (Nesting/Brooding) }\end{array}$} & V1: Mean VOR - Landscape (all vegetation including residual) & 0.45 & 0.37 & 162.09 & Nesting HSI \\
\hline & & & V2: Percent Slope & 0.30 & 0.72 & & Brood HSI \\
\hline & & & V3: Percent Cover Grass & 0.93 & & & \\
\hline & & & V4: Percent Cover Forbs & 0.35 & 0.52 & & Nest/Brood HSI \\
\hline & & & V5: Percent Cover Introduced Herbaceous Species & 0.84 & & & \\
\hline & & \multirow{2}{*}{ Landscape variables } & V6: Percent Equivalent Optimum Area Providing Nest/Brood Cover & 1.00 & & & \\
\hline & & & V7: Distance Between Nesting/Brood Rearing and Winter Habitat & 1.00 & & & \\
\hline \multirow{3}{*}{ Deciduous Shrub/Tree } & \multirow{3}{*}{6.40} & \multirow{2}{*}{ Sharp-tailed Grouse (Winter) } & V8: Percent Cover Deciduous shrubs and Trees & 0.15 & 0.05 & 0.33 & \\
\hline & & & V9: Deciduous Shrub and Tree Composition/Wheat Availability & 0.80 & & & \\
\hline & & Landscape variable & V10: Percent Equivalent Optimum Area Providing Winter Habitat & 0.15 & & & \\
\hline Total & 320.50 & & & & & 348.79 & \\
\hline
\end{tabular}


West Foster Creek Expansion Project

Table 8. Middle Bridgeport 2007 baseline HEP results summary.

Cover Type

Model/Comments

\begin{tabular}{|c|c|c|c|c|}
\hline Variable & SI & HSI & HUs & Remarks \\
\hline V1: \% C.C. Herb. Plants & 0.89 & 0.34 & 55.19 & \\
\hline V2: \% Herb. C.C. Composed of Grass & 0.97 & & & \\
\hline V3: Ave. Ht. of Herb. Canopy & 0.62 & & & \\
\hline V4: Distance to Perch Sites & 1.00 & & & \\
\hline V5: \% Shrub Canopy Cover & 0.46 & & & \\
\hline $\begin{array}{l}\text { V1: Percent cover of preferred } \\
\text { shrubs }<1.5 \text { meters in height }\end{array}$ & 0.66 & 0.40 & 65.00 & Food index \\
\hline V2: Percent cover of all shrubs $<1.5$ meters in height. & 0.66 & 0.56 & & Cover index \\
\hline V3: Mean shrub height. & 0.52 & 0.40 & & Final HSI \\
\hline V4: Number of preferred shrub species. & 0.63 & & & \\
\hline V5: Percent cover of palatable herbaceous species. & 1.00 & & & \\
\hline $\begin{array}{l}\text { V6: Presence of suitable agricultural crops } \\
\text { within } 1.6 \text { kilometers ( } 1 \text { mile) of study area }\end{array}$ & 0.10 & & & \\
\hline V7: Aspect & 0.42 & & & \\
\hline V8: Road density & 1.00 & & & \\
\hline V9: Topographic diversity & 0.70 & & & \\
\hline $\begin{array}{l}\text { V10: Percent evergreen canopy } \\
>1.5 \text { meters in height }\end{array}$ & 0.00 & & & \\
\hline V1: Mean VOR - Landscape (all vegetation including residual) & 0.37 & 0.49 & 103.22 & Nesting HSI \\
\hline V2: Percent Slope & 0.66 & 0.82 & & Brood HSI \\
\hline V3: Percent Cover Grass & 1.00 & & & \\
\hline V4: Percent Cover Forbs & 0.42 & 0.64 & & Nest/Brood HSI \\
\hline V5: Percent Cover Introduced Herbaceous Species & 0.92 & & & \\
\hline V6: Percent Equivalent Optimum Area Providing Nest/Brood Cover & 1.00 & & & \\
\hline V7: Distance Between Nesting/Brood Rearing and Winter Habitat & 1.00 & & & \\
\hline \multicolumn{5}{|l|}{ V8: Percent Cover Deciduous shrubs and Trees } \\
\hline \multicolumn{5}{|l|}{ V9: Deciduous Shrub and Tree Composition/Wheat Availability } \\
\hline \multicolumn{5}{|l|}{ V10: Percent Equivalent Optimum Area Providing Winter Habitat } \\
\hline & & & 223.40 & \\
\hline
\end{tabular}

Totals Acres

Shrubsteppe/Grassland

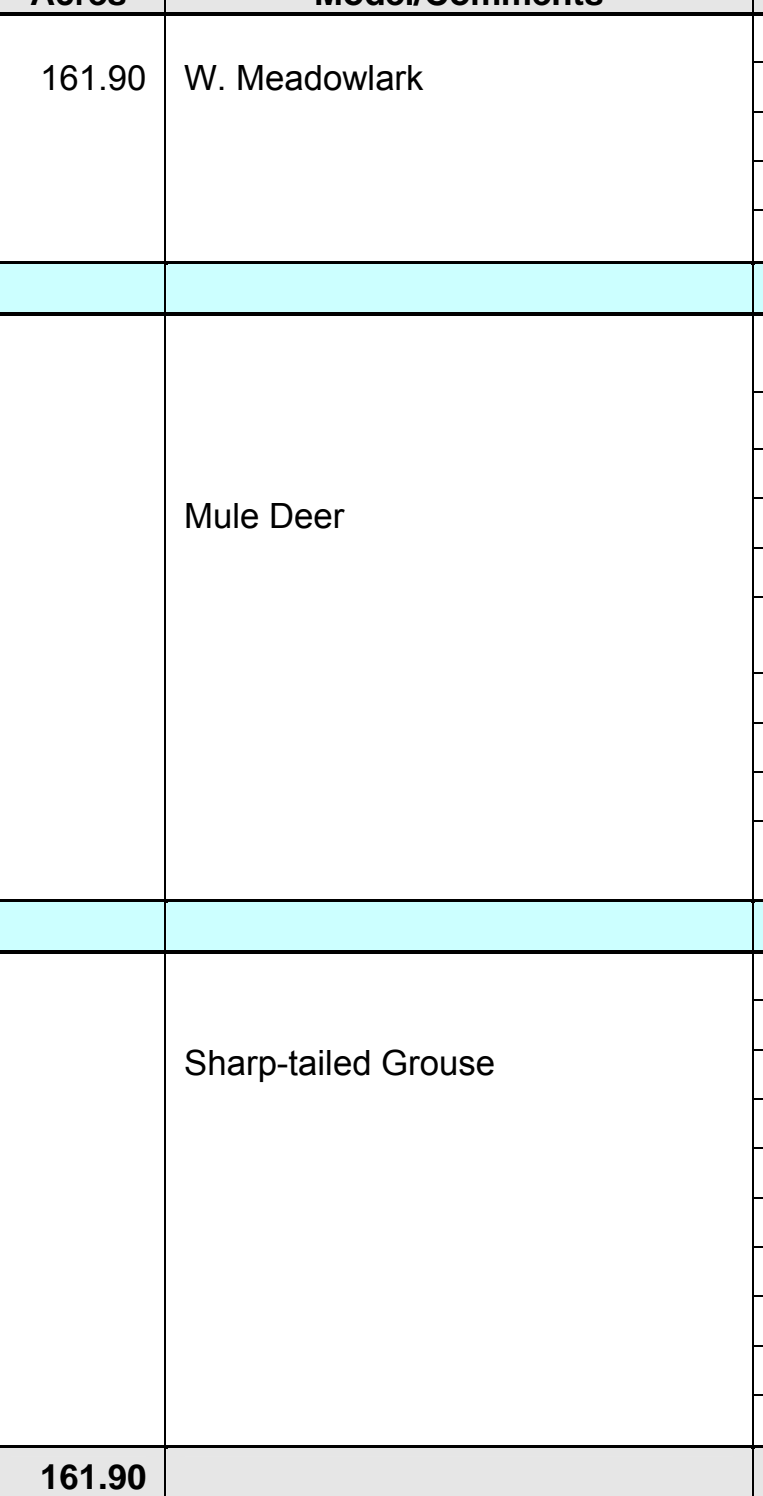

BPA receives full credit for the West Foster Creek Middle parcel (WDFW acquisition) for agreeing to withdraw the Clemons Mountain Unit and associated habitat units from the Wenas Wildlife Area mitigation project 
West Foster Creek Expansion Project

Table 9. McClain Lake 2007 baseline HEP results summary.

\begin{tabular}{|c|c|c|c|c|c|c|c|c|c|c|c|c|}
\hline \multirow{2}{*}{ Cover Type } & \multirow{2}{*}{ Acres } & \multirow{2}{*}{ Model/Comments } & \multirow{2}{*}{ Variable } & \multicolumn{4}{|c|}{ Shrubsteppe } & \multicolumn{4}{|c|}{ Grassland } & \multirow{2}{*}{$\begin{array}{l}\text { Total } \\
\text { HUs }\end{array}$} \\
\hline & & & & SI & HSI & HUs & & SI & HSI & HUs & $\begin{array}{l}\text { Remark } \\
s\end{array}$ & \\
\hline & & \multirow{5}{*}{ W. Meadowlark } & V1: \% C.C. Herb. Plants & 0.96 & 0.34 & 120.05 & & 1.00 & 0.49 & 43.74 & & 163.80 \\
\hline Shrubsteppe (est.) & 354.26 & & V2: \% Herb. C.C. Composed of Grass & 0.83 & & & & 1.00 & & & & \\
\hline \multirow[t]{25}{*}{ Grassland (est.) } & 90.00 & & V3: Ave. Ht. of Herb. Canopy & 0.61 & & & & 0.88 & & & & \\
\hline & & & V4: Distance to Perch Sites & 1.00 & & & & 0.75 & & & & \\
\hline & & & V5: \% Shrub Canopy Cover & 0.49 & & & & 0.60 & & & & \\
\hline & & & & & & & & & & & & \\
\hline & & \multirow{6}{*}{ Mule Deer } & $\begin{array}{l}\text { V1: Percent cover of preferred } \\
\text { shrubs }<1.5 \text { meters in height }\end{array}$ & 0.61 & 0.40 & 141.09 & Food Index & 0.00 & 0.12 & 10.86 & $\begin{array}{l}\text { Food } \\
\text { Index }\end{array}$ & 151.94 \\
\hline & & & V2: Percent cover of all shrubs $<1.5$ meters in height. & 0.61 & 0.40 & & Cover Index & 0.00 & 0.40 & & $\begin{array}{l}\text { Cover } \\
\text { Index }\end{array}$ & \\
\hline & & & V3: Mean shrub height. & 0.31 & & & & 0.00 & & & & \\
\hline & & & V4: Number of preferred shrub species. & 0.73 & & & & 0.00 & & & & \\
\hline & & & V5: Percent cover of palatable herbaceous species. & 0.84 & & & & 0.00 & & & & \\
\hline & & & $\begin{array}{l}\text { V6: Presence of suitable agricultural crops } \\
\text { within } 1.6 \text { kilometers ( } 1 \text { mile) of study area }\end{array}$ & 0.10 & & & & 0.10 & & & & \\
\hline & & \multirow[t]{4}{*}{ (estimated/weighted) } & V7: Aspect & 0.60 & & & & 0.60 & & & & \\
\hline & & & V8: Road density ${ }^{2}$ & 0.90 & & & & 0.70 & & & & \\
\hline & & & V9: Topographic diversity & 0.50 & & & & 0.50 & & & & \\
\hline & & & $\begin{array}{l}\text { V10: Percent evergreen canopy } \\
>1.5 \text { meters in height }\end{array}$ & 0.00 & & & & 0.00 & & & & \\
\hline & & \multirow{8}{*}{ Sharp-tailed Grouse } & & & & & & & & & Nesting & \\
\hline & & & V1: Mean VOR - Landscape (all vegetation including residual) & 0.62 & 0.79 & 262.18 & Nesting HSI & 1.00 & 0.99 & 83.15 & HSI & 345.33 \\
\hline & & & V2: Percent Slope & 1.00 & 0.70 & & Brood HSI & 0.98 & 0.86 & & $\begin{array}{l}\text { Drood } \\
\text { HSI }\end{array}$ & \\
\hline & & & V3: Percent Cover Grass & 0.95 & 0.74 & & $\begin{array}{l}\text { Nest/Brood HSI } \\
\text { (Transect HSI) }\end{array}$ & 1.00 & 0.92 & \multicolumn{2}{|c|}{$\begin{array}{l}\text { Nest/Brood HSI } \\
\text { (Transect HSI) }\end{array}$} & \\
\hline & & & V4: Percent Cover Forbs & 0.45 & & & & 0.47 & & & & \\
\hline & & & V5: Percent Cover Introduced Herbaceous Species & 0.86 & & & & 1.00 & & & & \\
\hline & & & V6: Percent Equivalent Optimum Area Providing Nest/Brood Cover & 1.00 & & & & 1.00 & & & & \\
\hline & & & V7: Distance Between Nesting/Brood Rearing and Winter Habitat & 0.75 & & & & 0.88 & & & & \\
\hline & & $\begin{array}{r}\text { (see deciduous } \\
\text { forest/shrub) }\end{array}$ & V8: Percent Cover Deciduous shrubs and Trees & N/A & & & & $\mathrm{N} / \mathrm{A}$ & & & & \\
\hline & & $\begin{array}{r}\text { (see deciduous } \\
\text { forest/shrub) }\end{array}$ & V9: Deciduous Shrub and Tree Composition/Wheat Availability & N/A & & & & $\mathrm{N} / \mathrm{A}$ & & & & \\
\hline & & (see deciduous & V10: Percent Equivalent Optimum Area Providing Winter Habitat & N/A & & & & N/A & & & & \\
\hline
\end{tabular}


West Foster Creek Expansion Project

\begin{tabular}{|c|c|c|c|c|c|c|c|c|c|}
\hline \multirow{3}{*}{ Cover Type } & & forest/shrub) & & & & & & & \multirow{3}{*}{$\begin{array}{l}\text { Total } \\
\text { HUs }\end{array}$} \\
\hline & \multirow{2}{*}{ Acres } & \multirow{2}{*}{ Model/Comments } & \multirow{2}{*}{ Variable } & \multicolumn{4}{|c|}{ Deciduous Forest/Shrub } & & \\
\hline & & & & SI & HSI & HUs & & & \\
\hline \multirow{3}{*}{$\begin{array}{l}\text { Deciduous- } \\
\text { Forest/Shrub }\end{array}$} & \multirow{3}{*}{14.50} & \multirow{2}{*}{$\begin{array}{l}\text { Sharp-tailed Grouse } \\
\text { (winter) }\end{array}$} & V8: Percent Cover Deciduous shrubs and Trees & 0.70 & 0.24 & 3.45 & & & 3.45 \\
\hline & & & V9: Deciduous Shrub and Tree Composition/Wheat Availability & 0.90 & & & & & \\
\hline & & Landscape variable & V10: Percent Equivalent Optimum Area Providing Winter Habitat & 0.30 & & & & & \\
\hline & & & & \multicolumn{4}{|c|}{ Lacustrine } & & \\
\hline \multirow[t]{3}{*}{ Lacustrine } & \multirow[t]{2}{*}{9.76} & \multirow[t]{2}{*}{ Mink } & V4: Percent shrubs and trees within $100 \mathrm{~m}$ of water & 0.15 & 0.21 & 2.07 & & & \\
\hline & & & V5: Shoreline development factor & 0.30 & & & & & \\
\hline & & & & \multicolumn{4}{|c|}{ Emergent Wetland } & & \\
\hline \multirow{3}{*}{ Emergent Wetland } & \multirow{3}{*}{0.67} & \multirow{3}{*}{ Mink } & V2: Percent of year with water present & 1.00 & 0.36 & 0.24 & & & \\
\hline & & & $\begin{array}{l}\text { V3: Percent of wetland basin dominated by persistent emergent. herbaceous } \\
\text { vegetation }\end{array}$ & 0.40 & & & & & \\
\hline & & & V4: Percent shrubs and trees within $100 \mathrm{~m}$ of water & 0.20 & & & & & \\
\hline Total & 469.19 & & & & & 526.77 & & 137.75 & 664.52 \\
\hline
\end{tabular}


West Foster Creek Expansion Project

Table 10. Dezellum Lake 2007 Baseline HEP survey results summary.

\begin{tabular}{|c|c|c|c|c|c|c|c|}
\hline Cover Type & Acres & Model/Comments & Variable & SI & HSI & HUs & Remarks \\
\hline \multirow{26}{*}{ Shrubsteppe/Grassland } & \multirow{5}{*}{156.58} & \multirow{5}{*}{ W. Meadowlark } & V1: \% C.C. Herb. Plants & 0.74 & 0.22 & 35.05 & \\
\hline & & & V2: \% Herb. C.C. Composed of Grass & 0.89 & & & \\
\hline & & & V3: Ave. Ht. of Herb. Canopy & 0.49 & & & \\
\hline & & & V4: Distance to Perch Sites & 1.00 & & & \\
\hline & & & V5: \% Shrub Canopy Cover & 0.39 & & & \\
\hline & & & & & & & \\
\hline & & \multirow{10}{*}{ Mule Deer } & $\begin{array}{l}\text { V1: Percent cover of preferred } \\
\text { shrubs }<1.5 \text { meters in height }\end{array}$ & 0.65 & 0.35 & 54.84 & Food Index \\
\hline & & & V2: Percent cover of all shrubs $<1.5$ meters in height. & 0.66 & 0.36 & & Cover Index \\
\hline & & & V3: Mean shrub height. & 0.26 & 0.35 & & Transect HSI \\
\hline & & & V4: Number of preferred shrub species. & 0.51 & & & \\
\hline & & & V5: Percent cover of palatable herbaceous species. & 0.89 & & & \\
\hline & & & $\begin{array}{l}\text { V6: Presence of suitable agricultural crops } \\
\text { within } 1.6 \text { kilometers ( } 1 \text { mile) of study area }\end{array}$ & 0.10 & & & \\
\hline & & & V7: Aspect & 0.60 & & & \\
\hline & & & V8: Road density & 0.80 & & & \\
\hline & & & V9: Topographic diversity & 0.45 & & & \\
\hline & & & $\begin{array}{l}\text { V10: Percent evergreen canopy } \\
>1.5 \text { meters in height }\end{array}$ & 0.00 & & & \\
\hline & & \multirow{10}{*}{ Sharp-tailed Grouse } & V1: Mean VOR - Landscape (all vegetation including residual) & 0.55 & 0.71 & 101.16 & Nesting HSI \\
\hline & & & V2: Percent Slope & 0.93 & 0.59 & & Brooding HSI \\
\hline & & & V3: Percent Cover Grass & 0.74 & & & \\
\hline & & & V4: Percent Cover Forbs & 0.39 & 0.65 & & \multirow{2}{*}{$\begin{array}{l}\text { Nest/Brood } \\
\text { HSI } \\
\text { (Transect } \\
\text { HSI) }\end{array}$} \\
\hline & & & V5: Percent Cover Introduced Herbaceous Species & 0.84 & & & \\
\hline & & & V6: Percent Equivalent Optimum Area Providing Nest/Brood Cover & 1.00 & & & \\
\hline & & & V7: Distance Between Nesting/Brood Rearing and Winter Habitat & 0.60 & & & \\
\hline & & & V8: Percent Cover Deciduous shrubs and Trees & N/A & & & \\
\hline & & & V9: Deciduous Shrub and Tree Composition/Wheat Availability & $\mathrm{N} / \mathrm{A}$ & & & \\
\hline & & & V10: Percent Equivalent Optimum Area Providing Winter Habitat & $\mathrm{N} / \mathrm{A}$ & & & \\
\hline Total & 156.58 & & & & & 191.05 & \\
\hline \multirow{3}{*}{ Emergent Wetland ${ }^{2}$} & \multirow{3}{*}{5.00} & \multirow{3}{*}{ Mallard } & V7: Percent emergent cover to percent open water ratio & 0.73 & 0.47 & 2.35 & \\
\hline & & & V8: Water Permanence & 0.30 & & & \\
\hline & & & V9: Presence/absence of carp & 1.00 & & & \\
\hline
\end{tabular}


West Foster Creek Expansion Project Cover Type

\begin{tabular}{c|r} 
& Acres \\
Total & $\mathbf{5 . 0 0}$
\end{tabular}

\section{Lacustrine $^{3}$} Total Acres
5.00 Model/Comments

\begin{tabular}{|l|l}
\multicolumn{1}{|c}{ Variable } \\
\hline V7: Percent emergent cover to percent open water ratio \\
\hline V8: Water Permanence \\
\hline V9: Presence/absence of carp \\
\hline
\end{tabular}

\begin{tabular}{|r|r|}
\hline \multicolumn{1}{|c|}{ SI } & HSI \\
\hline 0.50 & 0.50 \\
\hline 0.50 & \\
\hline 1.00 & \\
\hline & \\
\hline
\end{tabular}

HUs

2.35

Dezellum Lake Total

Total

5.00 Mallard

166.58 All species

BPA receives full credit for the Dezellum Lake parcel (WDFW acquisition) for agreeing to withdraw the Clemons Mountain Unit and associated habitat units from the Wenas Wildlife Area mitigation project.

${ }^{2}$ Emergent wetlands are ephemeral and estimated at approximately 5 acres (D. Peterson, WDFW, pers. comm.)

${ }^{3}$ Dezellum Lake and one other pond, located in the sw corner of the property, are permanent water and estimated to be approximately 5 acres (D. Peterson, WDFW, pers. comm.). 
West Foster Creek Expansion Project

Table 11. JoJaCo 2007 baseline HEP survey results summary.

\begin{tabular}{|c|c|c|c|c|c|c|c|c|}
\hline Cover Type & Acres & Model/Comments & Variable & SI & HSI & HUs & Reme & \\
\hline \multirow{22}{*}{ Shrubsteppe/Grassland } & \multirow{22}{*}{$2,593.31$} & \multirow{5}{*}{ W. Meadowlark } & V1: \% C.C. Herb. Plants & 0.89 & 0.45 & $1,199.99$ & & \\
\hline & & & V2: \% Herb. C.C. Composed of Grass & 1.00 & & & & \\
\hline & & & V3: Ave. Ht. of Herb. Canopy & 0.90 & & & & \\
\hline & & & V4: Distance to Perch Sites & 1.00 & & & & \\
\hline & & & V5: \% Shrub Canopy Cover & 0.50 & & & & \\
\hline & & \multirow{10}{*}{ Mule Deer } & $\begin{array}{l}\text { V1: Percent cover of preferred } \\
\text { shrubs }<1.5 \text { meters in height }\end{array}$ & 0.56 & 0.35 & 907.66 & WFI & 0.35 \\
\hline & & & V2: Percent cover of all shrubs $<1.5$ meters in height. & 0.56 & & & $\mathrm{WCl}$ & 0.80 \\
\hline & & & V3: Mean shrub height. & 0.45 & & & & \\
\hline & & & V4: Number of preferred shrub species. & 0.71 & & & & \\
\hline & & & V5: Percent cover of palatable herbaceous species. & 0.97 & & & & \\
\hline & & & $\begin{array}{l}\text { V6: Presence of suitable agricultural crops } \\
\text { within } 1.6 \text { kilometers ( } 1 \text { mile) of study area }\end{array}$ & 0.10 & & & & \\
\hline & & & V7: Aspect & 0.40 & & & & \\
\hline & & & V8: Road density & 1.00 & & & & \\
\hline & & & V9: Topographic diversity & 1.00 & & & & \\
\hline & & & $\begin{array}{l}\text { V10: Percent evergreen canopy } \\
>1.5 \text { meters in height }\end{array}$ & 0.00 & & & & \\
\hline & & \multirow{7}{*}{ Sharp-tailed Grouse } & V1: Mean VOR - Landscape (all vegetation including residual) & 0.64 & 0.54 & $1,400.39$ & \multicolumn{2}{|c|}{ Nest/brood HSI } \\
\hline & & & V2: Percent Slope ${ }^{2}$ & 0.50 & 0.51 & & Brood HSI & \\
\hline & & & V3: Percent Cover Grass & 0.99 & 0.57 & & $\begin{array}{l}\text { Nesting } \\
\text { HSI }\end{array}$ & \\
\hline & & & V4: Percent Cover Forbs & 0.39 & & & & \\
\hline & & & V5: Percent Cover Introduced Herbaceous Species & 0.58 & & & & \\
\hline & & & V6: Percent Equivalent Optimum Area Providing Nest/Brood Cover & 1.00 & & & & \\
\hline & & & V7: Distance Between Nesting/Brood Rearing and Winter Habitat & 1.00 & & & & \\
\hline Total & $2,593.31$ & & & & & $3,508.04$ & & \\
\hline \multirow{3}{*}{ Riparian Shrub } & \multirow{3}{*}{8.00} & \multirow{3}{*}{ Sharp-tailed Grouse } & V8: Percent Cover Deciduous shrubs and Trees & 1.00 & 0.04 & 0.32 & & \\
\hline & & & V9: Deciduous Shrub and Tree Composition/Wheat Availability & 0.63 & & & & \\
\hline & & & V10: Percent Equivalent Optimum Area Providing Winter Habitat & 0.05 & & & & \\
\hline Total & 8.00 & & & & & 0.32 & & \\
\hline Riparian Forest & 22.00 & Sharp-tailed Grouse & V8: Percent Cover Deciduous shrubs and Trees & 1.00 & 0.04 & 0.90 & & \\
\hline
\end{tabular}


West Foster Creek Expansion Project

\begin{tabular}{|c|c|c|c|c|c|c|c|}
\hline Cover Type & Acres & Model/Comments & Variable & SI & HSI & HUs & Remarks \\
\hline & & & V9: Deciduous Shrub and Tree Composition/Wheat Availability & 0.67 & & & \\
\hline & & & V10: Percent Equivalent Optimum Area Providing Winter Habitat & 0.05 & & & \\
\hline Total & 22.00 & & & & & 0.90 & \\
\hline \multirow{3}{*}{ Conifer Woodland } & \multirow{3}{*}{7.00} & \multirow{3}{*}{ Black-capped Chickadee } & V1: Percent Tree Canopy Closure & 1.00 & 0.50 & 3.50 & \\
\hline & & & V2: Average Height of Overstory Trees & 0.90 & & & \\
\hline & & & V4: Number of snags 4" to 10" DBH per Acre & 0.50 & & & \\
\hline Total & 7.00 & & & & & 3.50 & \\
\hline \multirow{4}{*}{ Rock, Cliff, Talus } & \multirow{4}{*}{8.00} & \multirow{4}{*}{ Bobcat } & V1: Percent cover herbaceous vegetation & 1.00 & 0.13 & 1.07 & \\
\hline & & & V2: Shrub distribution & 0.80 & & & \\
\hline & & & V3: Percent shrub cover & 0.30 & & & \\
\hline & & & V4: Percent area comprised of rock outcrops, boulders, etc. & 0.05 & & & \\
\hline Total & 8.00 & & & & & 1.07 & \\
\hline Parcel Total & $2,638.31$ & & & & & $3,513.83$ & \\
\hline & & & & & & & \\
\hline \multicolumn{8}{|c|}{ BPA receives full credit for this parcel (WDFW acquisition) for agreeing to withdraw the Clemons Mountain Unit and associated habitat units from the Wenas Wildlife Area mitigation project. } \\
\hline $\begin{array}{l}\text { The percent slope suitability index (SI) was re } \\
\text { owards areas with less slope. }\end{array}$ & duced to more & accurately reflect project landscap & conditions. Actual transect results rated this variable as 0.76 (see & & tra & ocations & to \\
\hline
\end{tabular}




\section{Discussion}

This section includes comments on HEP survey results for individual parcels. In general, HEP model HSI results were similar at all sites.

\section{North Bridgeport}

The Regional HEP Team established 13 transects on the shrubsteppe and deciduous shrub/tree cover types on the North Bridgeport parcel. All transects were 300 feet in length.

\section{Shrubsteppe}

\section{Western Meadowlark}

Habitat suitability for western meadowlark was "poor" (0.16 HSI) largely because of the relatively high percent cover of shrub species and less than optimum height of herbaceous vegetation. Reducing shrub cover and replacing invasive herbaceous grass species such as cheatgrass with native bunchgrasses would increase habitat suitability for this species.

\section{Mule Deer}

The winter mule deer model HSI output was 0.43 or in the low "fair" range. Aspect, which cannot be changed through management actions, was the most limiting model variable. It could be argued, however, that aspect may not have as significant an impact at this site (as projected by the model variable curve) due to its location adjacent to Lake Roosevelt. The river influences the local micro climate resulting in less severe winter temperatures and snow depth.

\section{Sharp-tailed Grouse}

Sharp-tailed grouse habitat suitability (nesting and brood rearing) was rated as "fair" (0.52 HSI). Visual obstruction or VOR (horizontal/vertical cover) was the primary limiting model variable. Increasing the density of native bunchgrasses would improve VOR/HSI. The relatively steep slope on much of the project site also contributed to reducing nesting habitat suitability. 


\section{Deciduous Shrub/Tree}

\section{Sharp-tailed Grouse}

Sharp-tailed grouse winter habitat suitability was rated "poor" $(0.05 \mathrm{HSI})$ due to low percent cover of shrubs and trees that provide winter food, and the limited amount of this habitat component at this site. Increasing the amount of winter habitat on this parcel is problematic due to edaphic ${ }^{11}$ constraints and limited wet sites; however, improving habitat quality on areas currently supporting shrubs and trees is a viable option.

\section{Middle Bridgeport}

The Regional HEP Team established 7 shrubsteppe transects on the Middle Bridgeport parcel. Transects were either 300 feet or 400 feet in length.

\section{Shrubsteppe}

\section{Western Meadowlark}

Habitat suitability for western meadowlark was "marginal" (0.34 HSI) largely because of the relatively high percent cover of shrub species and less than optimum height of herbaceous vegetation. Reducing shrub cover and increasing native bunchgrass cover would increase habitat suitability for this species.

\section{Mule Deer}

The winter mule deer model HSI output was 0.40 or in the low "fair" range. Limiting factors included relatively low habitat suitability for percent cover of preferred shrubs and landscape aspect, which cannot be changed through management actions.

\section{Sharp-tailed Grouse}

Sharp-tailed grouse habitat suitability (nesting and brood rearing) was rated as "fair" (0.64 HSI). Visual obstruction or VOR (horizontal/vertical cover) was the primary limiting biotic factor. Increasing the density of native bunchgrasses would result in improved VOR/HSI. Based on HEP model output, this site provided adequate nesting and brood rearing habitat.

\section{McClain Lake}

McClain Lake was the only parcel surveyed where shrubsteppe was separated into its basic components i.e., shrubland (identified as shrubsteppe in this section) and grassland or "steppe." The RHT collected data on 12 measured transects and one ocular transect (measured transects were 300 feet in length).

\footnotetext{
${ }^{11}$ Edaphic features are abiotic factors associated with soils e.g., PH, soil depth, rock, etc.
} 


\section{Shrubsteppe}

\section{Western Meadowlark}

Habitat suitability for western meadowlark was "marginal" ( 0.34 HSI) because of the relatively high percent cover of shrub species ${ }^{12}$ and less than optimum height of herbaceous vegetation. As discussed for other WFCEP sites, reducing shrub cover and replacing invasive herbaceous grass species such as cheatgrass with native bunchgrasses would increase habitat suitability for this species.

\section{Mule Deer}

The winter mule deer model HSI output was 0.40 or in the low "fair" range. Percent cover of preferred shrubs and shrub height (security cover) were the primary factors limiting the winter "food" HSI. The winter cover index (WCI) was equally low (0.40) due to limited topographic relief and the lack of an evergreen over-story greater than five feet in height. Edaphic features and the inability to modify the topography likely will limit future habitat suitability to current ratings.

\section{Sharp-tailed Grouse}

Sharp-tailed grouse habitat suitability (nesting and brood rearing) was rated as "good" (0.72 HSI). No immediate action is necessary to improve nesting and brood rearing habitat.

\section{Grassland}

\section{Western Meadowlark}

Habitat suitability for western meadowlark was rated only "fair" ( $0.49 \mathrm{HSI})$ because of the relatively high percent cover of shrub species. All other model variables were near optimum conditions.

\section{Mule Deer}

As expected, the winter mule deer model HSI output was 0.12 or in the "poor" range. The general lack of shrubs was the principle reason the HSI was low. If WDFW continues to maintain the grassland sites as grassland habitat, the winter mule deer model HSI output will not change in the future, because shrub browse/cover variables "drive" the winter food index (WFI) portion of the HSI model.

\footnotetext{
${ }^{12}$ Percent shrub cover is a threshold variable that has a significant impact on the final HSI. Shrub cover $\geq$ $35 \%$ reduces the HSI to 0.0 . Personal observation shows that shrub cover may not be as large a limiting factor as indicated in the HSI model. The most dense western meadowlark population I have observed occupied a sagebrush site with $>40 \%$ shrub cover that also supported a robust, intact, native bunchgrass plant community (P. Ashley, pers. comm.).
} 


\section{Sharp-tailed Grouse}

Sharp-tailed grouse habitat suitability (nesting and brood rearing) was rated nearly "optimum" (0.92 HSI). No immediate action is necessary to improve nesting and brood rearing habitat.

\section{Deciduous Shrub/Tree}

\section{Sharp-tailed Grouse}

Sharp-tailed grouse winter habitat suitability was rated "marginal" (0.24 HSI) due to the limited amount of this habitat component at this site. Increasing the amount of winter habitat on this parcel is problematic due to edaphic constraints and limited wet sites; however, improving habitat quality on areas currently supporting shrubs and trees is likely possible.

\section{Lacustrine (Lake)}

\section{Mink}

The mink HEP model was used to determine lacustrine cover type habitat quality and HUs. Shrub and tree cover within 100 meters of the shoreline and shoreline development were the two habitat variables evaluated to determine habitat suitability. The "marginal" HSI (0.21) rating was the result of low shrub and tree cover within 100 meters of the shoreline. Similar to other areas, edaphic features limit the amount of shoreline cover present.

\section{Emergent Wetland}

\section{Mink}

Like the lacustrine cover type, the low percent shrub and tree cover within 100 meters of the shoreline resulted in a "marginal" HSI (0.36) rating. Again, edaphic features limit the amount of shoreline cover present and future potential.

\section{Dezellum Lake}

The Regional HEP team established 13 transects on the Dezellum Lake parcel. Eight transects were measured (300 feet in length) while five transects were "ocular"; primarily associated with lacustrine and emergent wetland sites.

\section{Shrubsteppe}

\section{Western Meadowlark}

Habitat suitability for western meadowlark was "marginal" (0.22 HSI) primarily due to the relatively high percent cover of shrub species and less than optimum height of 
herbaceous vegetation. Reducing shrub cover and replacing invasive herbaceous grass species such as cheatgrass with native bunchgrasses would increase habitat suitability for this species.

\section{Mule Deer}

The 0.35 WFI and 0.36 WCI suggest that both mule deer winter food and cover are limiting factors on the Dezellum parcel. Percent cover of preferred shrubs and shrub height (security cover) are the limiting factors relative to the "food" portion of the winter mule deer HEP model, while the lack of topographic diversity and evergreen over-story (thermal cover) are responsible for the low winter cover suitability index rating.

\section{Sharp-tailed Grouse}

Sharp-tailed grouse habitat suitability (nesting and brood rearing) was rated as "good" (0.65 HSI). Based on model output, visual obstruction or VOR (horizontal/vertical cover) was the primary limiting factor. Increasing the density of native bunchgrasses would improve VOR/HSI.

\section{Lacustrine}

\section{Mallard}

The mallard HSI was rated "fair" (0.50 HSI). Percent emergent cover to open water ratio and water permanence was the two limiting model variables (emergent vegetation was lacking and open water was permanent; both reduced the HSI).

\section{Emergent Wetland}

\section{Mallard}

Unlike the lacustrine cover type, percent emergent cover to open water ratio suitability did not limit the HSI in this cover type. The factor most responsible for the "fair" $(0.47$ HSI) assessment was the limited time water was present on ephemeral wetlands (in recent drought years water was available approximately five months annually; however, water may be present longer in wet years (D. Peterson, pers. comm.).

\section{JoJaCo}

The Regional HEP Team established 26 transects on the JoJaCo parcel. Transect length ranged from 150 feet to 600 feet. 


\section{Shrubsteppe}

\section{Western Meadowlark}

Habitat suitability for western meadowlark was "fair" (0.45 HSI). As at other sites, the relatively high percent cover of shrub species limited habitat suitability.

\section{Mule Deer}

The winter mule deer model HSI output was 0.35 or in the "marginal" range. The lack of preferred shrub cover (browse) and aspect, which cannot be changed through management actions, were the model variables most responsible for the low HSI rating.

\section{Sharp-tailed Grouse}

Sharp-tailed grouse habitat suitability (nesting and brood rearing) was rated as "fair" (0.54 HSI). Visual obstruction or VOR (horizontal/vertical cover) was the primary limiting model variable. The steepness of the slope and percent cover of introduced species also contributed to reducing habitat suitability.

\section{Riparian Shrub}

\section{Sharp-tailed Grouse}

The winter component of the sharp-tailed grouse model was used to evaluate the riparian shrub cover type. The HSI was 0.04 (poor) because of the limited amount of riparian shrub cover type present on this parcel. Increasing the amount of riparian shrub may be problematic because of edaphic features and limited mesic sites. At the landscape level, sharp-tailed grouse winter habitat may not be as limiting as suggested by the HEP model output, because the grouse are mobile and winter habitat does exist on adjacent, nonproject lands.

\section{Riparian Forest}

\section{Sharp-tailed Grouse}

Like riparian shrub, habitat suitability was "poor" (0.04 HSI) due to the limited amount of riparian shrub cover type present on this parcel. Increasing the amount of riparian forest may be problematic because of edaphic features and limited mesic sites.

\section{Conifer Woodland}

\section{Black-capped Chickadee}

The black-capped chickadee HEP model was used to evaluate the conifer woodland cover type. This cover type was rated $0.50 \mathrm{HSI}$ or "fair." The lack of snags 4 " to 10" DBH was the limiting factor. 


\section{Rock/Cliff/Talus}

\section{Bobcat}

Habitat suitability for the bobcat was rated "poor" (0.13 HSI). The lack of shrub cover within and immediately adjacent to this cover type and low acreage were the factors that limited the HSI output.

\section{Acknowledgements}

I gratefully acknowledge the hard work and effort provided by WDFW Sagebrush Flat Wildlife Area Assistant Manager Dan Peterson and Regional HEP Team members Mikael Cantonese, Tiffany Baker, Tony Muse, and Paul Walker. I also want to thank John Talmadge (WDFW) for providing GIS support and Dan Budd (WDFW Real Estate Division) for responding to a myriad of acquisition related questions in a timely manner. A special thank you goes to WDFW Wildlife Area Manager Marc Hallet for addressing numerous questions. 


\section{References}

Allen, A. W. 1984. Habitat suitability index models:

Mink (Revised May 1984). U.S. Department of the Interior, Fish and Wildlife Service. FWS/OBS-82/10.38.

Ashley, P. R., and M. Berger 1999. Habitat suitability model mule deer (winter). Olympia WA: Washington Department of Fish and Wildlife. Nespelem, WA: Colville Confederated Tribes.

Ashley, P. R. 2003. Sharp-tailed grouse HEP model. Olympia WA: Washington Department of Fish and Wildlife.

.2006. Habitat evaluation procedures standard measurement protocols and techniques (draft). Columbia Basin Fish and Wildlife Authority (CBFWA). Portland, OR.

Avery, T.E., H. E. Burkhart. 1994. Forest measurements. $4^{\text {th }}$ edition. New York, NY: John Wiley and Sons.

Berger, M. T. and D. Kuehn. 1992. Wildlife impact assessment Chief Joseph Dam Project. Project N0. 88-44. Bonneville Power Administration. Portland, OR.

BPA/WDFW. 1996. Memorandum of Agreement between the Washington Department of Fish and Wildlife and Bonneville Power Administration for the disbursal of wildlife mitigation funds and mitigation crediting. WDFW. Olympia, WA. BPA. Portland, OR.

Gotelli, N. J., A. M. Ellison. 2004. A primer of ecological statistics. Sinauer Associates, Inc. Sunderland, MA.

Hays, R. L., C. Summers, and W. Seitz. 1981. Estimating habitat variables. Western Energy and land Use Team. Fort Collins, CO: U.S. Fish and Wildlife Service.

Howerton, J., J. Creveling, and B. Renfrow. 1986. Wildlife protection, mitigation, and enhancement planning for Grand Coulee Dam. Olympia, WA: Washington Department of Fish and Wildlife.

Husch, B., T.W. Beers, and J.A. Kershaw, Jr. 2003. Forest mensuration- $4^{\text {th }}$ edition. Hoboken, NJ: Wiley and Sons, Inc.

Ohmann, J. L., M. J. Gregory, and J. S. Fried. 2006. The Pacific Northwest Regional GAP Analysis Project. Final report on Mapping of forest ecological systems of map zones 8 and 9 with gradient nearest neighbor imputation. Gap Analysis 
Program-Biological Resources Division, U.S. Geolical Survey. Pacific Northwest Research Station. USDA Forest Service. Corvallis, OR.

Robel, R.J., J. N. Dayton, A.D. Hulbert. 1975. Relationship between visual obstruction measurements and weight of grassland vegetation. Journal of Range Management. 23: 295.

Schroeder, R.L. 1983. Habitat suitability index models:

Black-capped chickadee. U.S. Department of the Interior, Fish and Wildlife Service. FWS/OBS-82/10.38.

Schroeder, R.L., and P.J. Sousa. 1982. Habitat suitability index models: Eastern meadowlark. U.S. Department of the Interior, Fish and Wildlife Service. FWS/OBS-82/10.29.

USFWS. 1980. Habitat as a Basis for Environmental Assessment, Ecological Services Manual (ESM) 101. Division of Ecological Services, U. S. Fish and Wildlife Service, Washington, DC: Department of the Interior. . 1980a. Habitat Evaluation Procedures (HEP), Ecological Services Manual (ESM) 102. Division of Ecological Services, U.S. Fish and Wildlife Service, Washington, DC: Department of the Interior.

WDFW. 1999. Columbia Basin Wildlife Area mallard HEP model. Washington Department of Fish and Wildlife. Olympia, WA. 2001. Sagebrush Flat Wildlife Area Work Plan Addendum - 2001. Washington Department of Fish and Wildlife. Olympia, WA. 


\section{Appendix A - Abbreviated HEP Models}

\section{Mule Deer}

V1: Percent palatable shrub cover

$<5 \mathrm{ft}$ in height

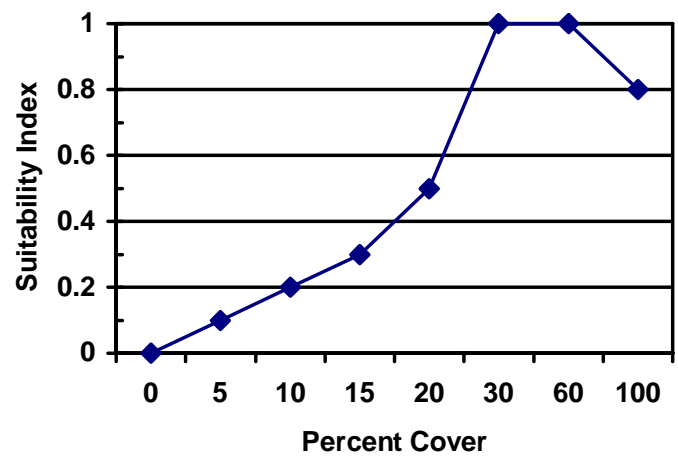

V2: Percent cover all shrubs $<5 \mathrm{ft}$ in height

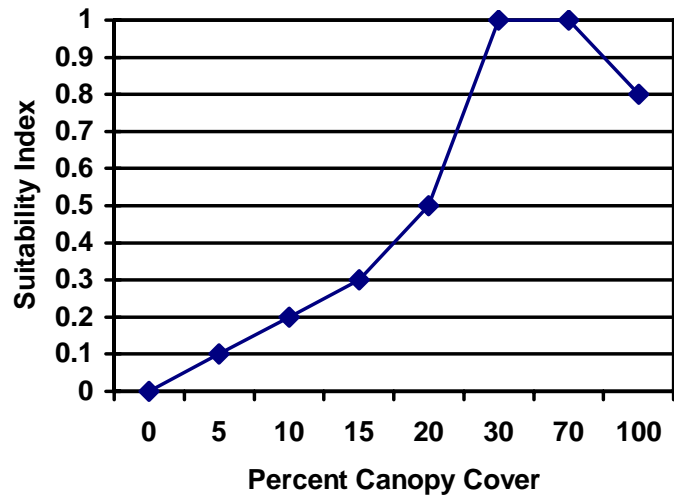

V3: Mean shrub height

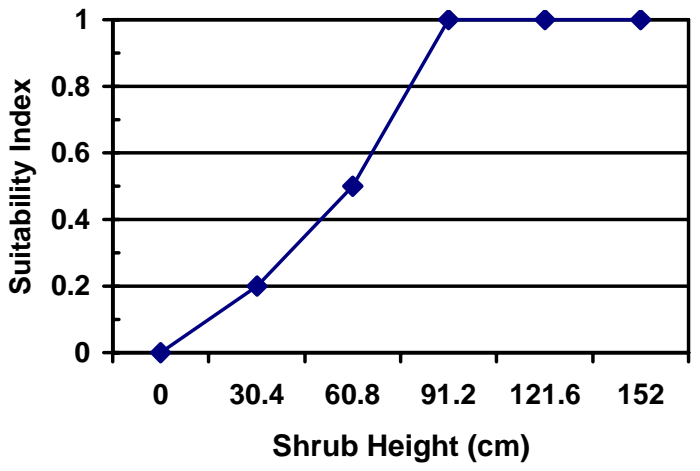


V4: No. of preferred shrub species

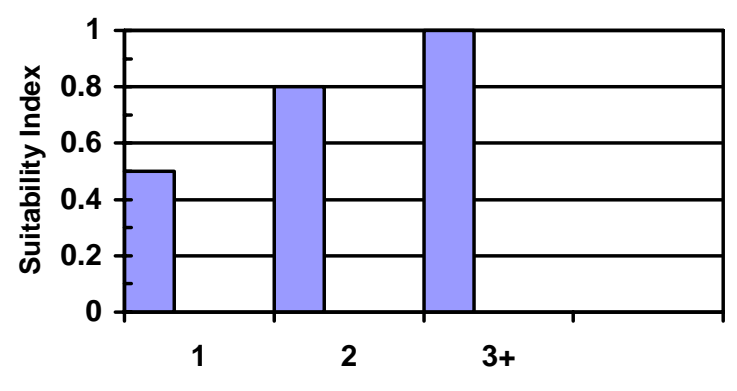

Number of Preferred Shrub Species

V5: Percent cover palatable herbaceous species

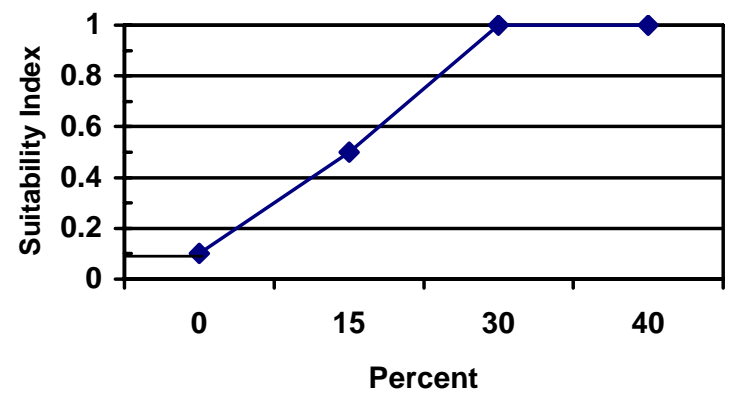

V6: Presence of suitable agricultural crops within 1.6 kilometers (1 mile) of study area Yes: 0.1

No: 0.0 
V7: Aspect

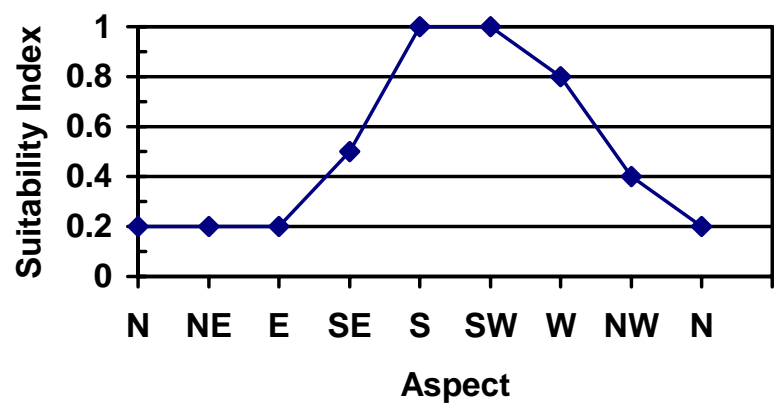

V8: Road density

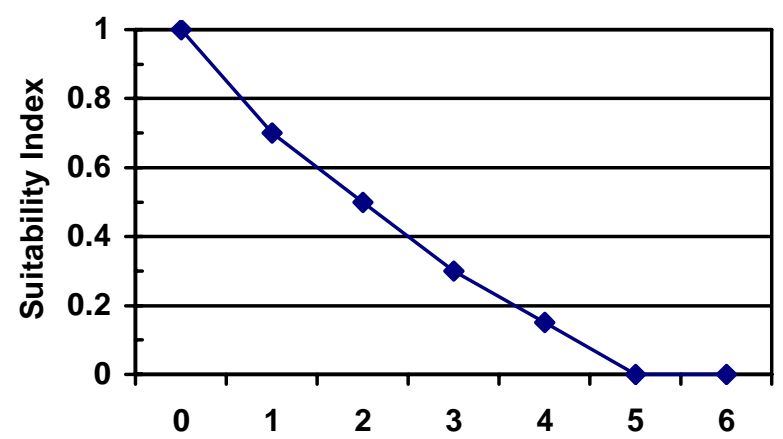

Kilometers of Open Road per Square Kilometer

V9: Topographic diversity

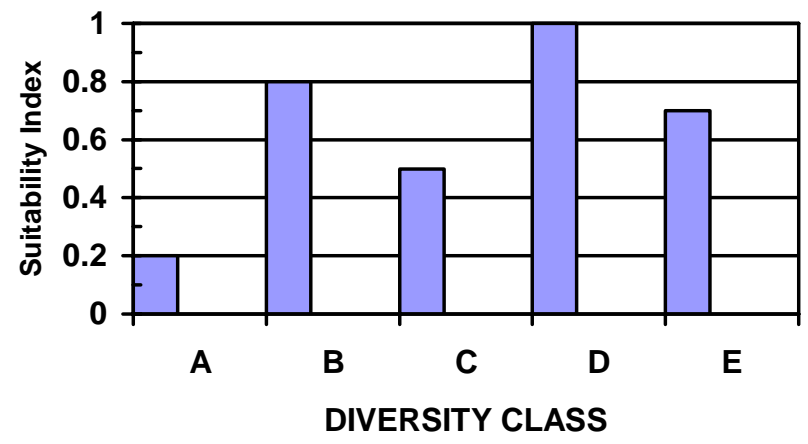


$\mathrm{V}_{9} \quad$ Topographic diversity.
A: Level terrain less than 5 percent slope.
B: Level terrain broken by drainages.
C: Rolling terrain 5 to 25 percent slope.
D: Rolling terrain with rims, ridges, and/or drainages.
E: Mountainous terrain with slopes greater than 25 percent. V10: Percent evergreen cover $>5 \mathrm{ft}$
in height

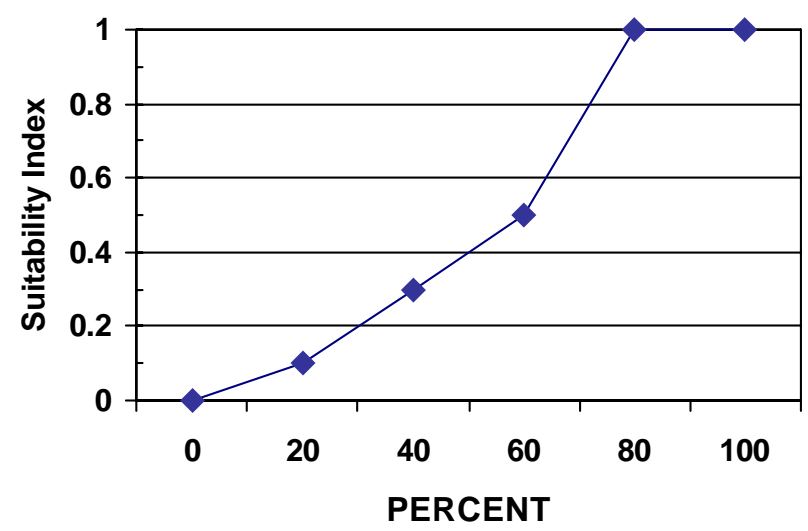

Shrubsteppe $\mathrm{HSI}=$ minimum value $\mathrm{WFI}$ or $\mathrm{WCI}$

WFI = ((V1 (V2 x V3 x V4 x V5) $\left.{ }^{1 / 4}\right)+$ V6) $x$ V7 $)^{\wedge} .625 \times$ V8

Steps in calculating WFI with a hand calculator:

1. Obtain geometric mean of V2, V3, V4, and V5

2. Multiply product from step one by V1 and add V6

3. Multiply sum obtained in step two by V7

4. Take the 1.66 root $(\wedge .6$ on your computer)of product from step 3

5. Multiply result from step 4 by V8 to obtain WFI

$\mathrm{WCI}_{S S}=(\mathrm{V9} \times .8)+\mathrm{V} 10$

Conifer Forest HSI = Lower Value Between:

WFI $=\left(\left(\left(V 1\left(V 2 \times \text { V3 } x \text { V4 } x \text { V5) }{ }^{1 / 4}\right)+\text { V6) } \times \text { V7 }\right)^{\wedge}{ }^{.625} \times\right.\right.$ V8

$\mathrm{WCI}_{\mathrm{F}}=2(\mathrm{~V} 10)+\mathrm{V} 9$ 
West Foster Creek Expansion Project

\section{Sharp-tailed Grouse}

V1: Mean VOR - Landscape (all vegetation including residual)

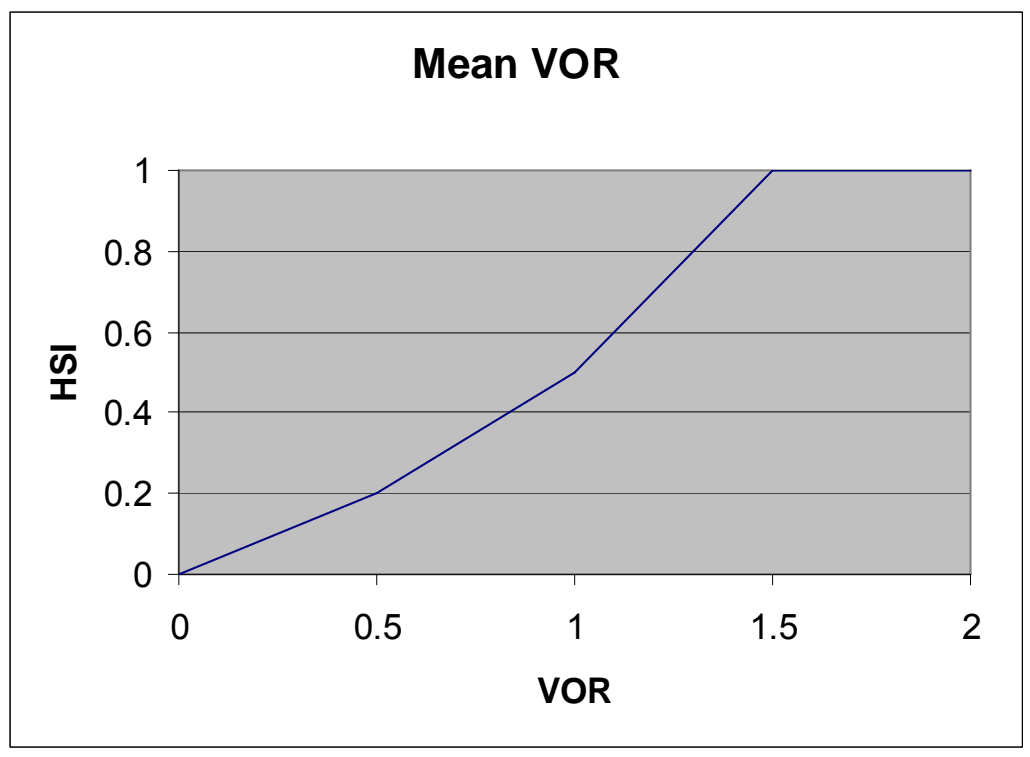

V2: Percent Slope

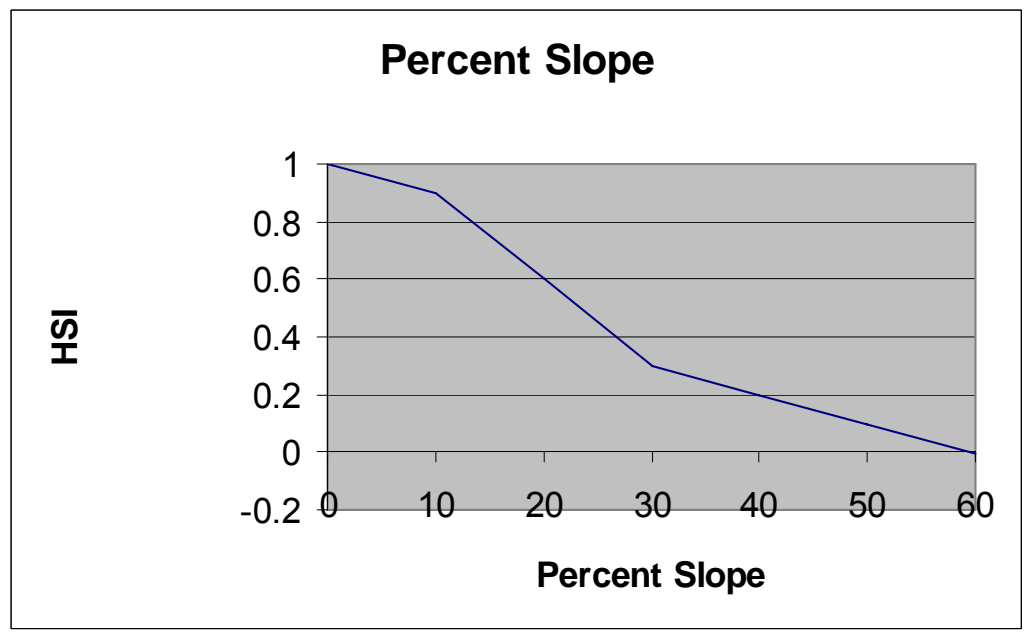

Nesting Habitat HSI Equation: $(\mathrm{V} 1 \times \mathrm{V} 2 \times \mathrm{V6})^{1 / 2}$ 
West Foster Creek Expansion Project

Brood Rearing Habitat

V3: Percent Cover Grass

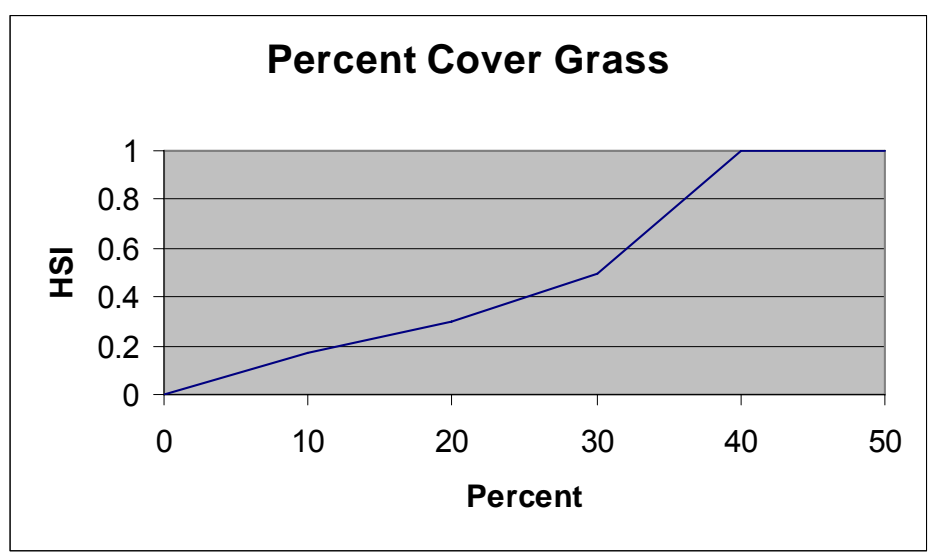

V4: Percent Cover Forbs

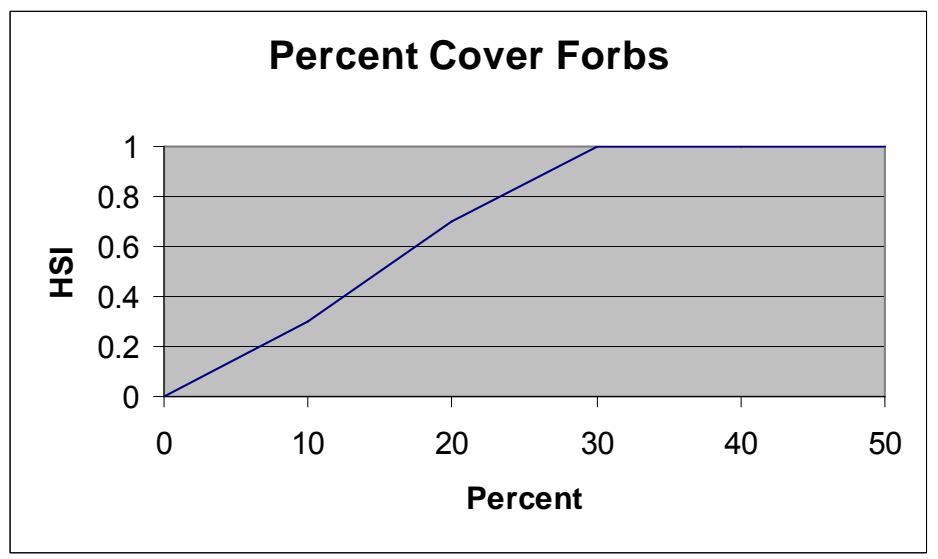

V5: Percent Cover Introduced Herbaceous Species

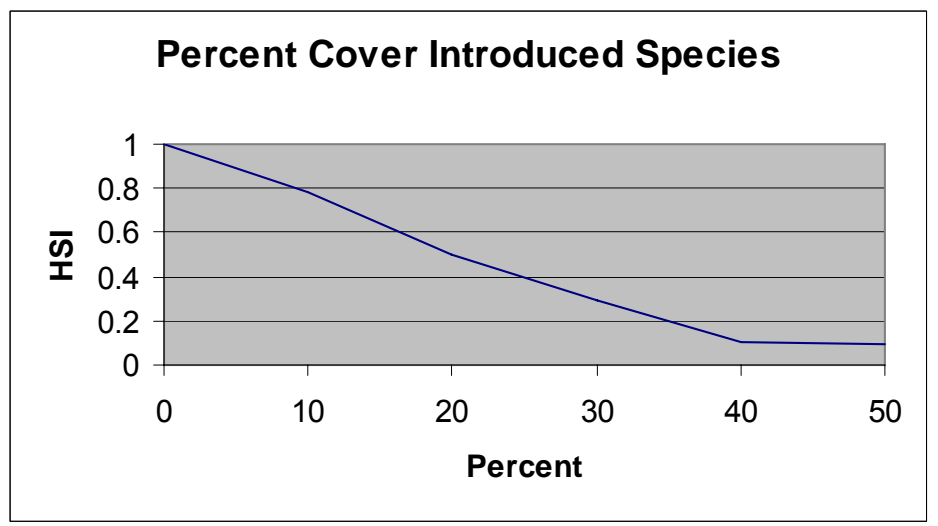


West Foster Creek Expansion Project

V7: Distance Between Nesting/Brood Rearing and Winter Habitat

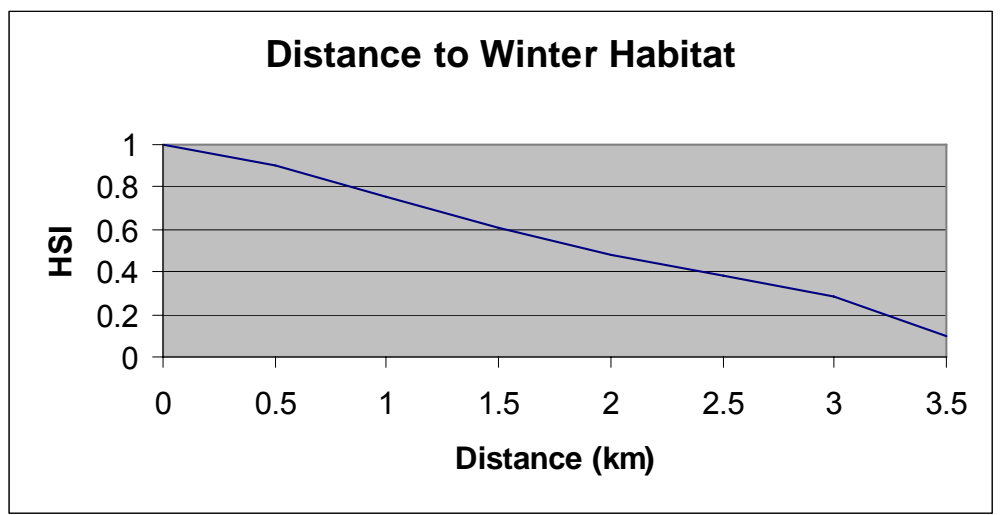

Brood Rearing HSI Equation: $\left[[((\mathrm{V} 3+\mathrm{V} 4) / 2)(\mathrm{V} 6)(\mathrm{V} 7)]^{1 / 3}(\mathrm{~V} 5)\right]$ Nesting/Brood Rearing HSI $=(\text { Nesting HSI } \times \text { Brood Rearing HSI })^{1 / 2}$

\section{Winter Habitat}

V8: Percent Cover Deciduous Shrubs and Trees

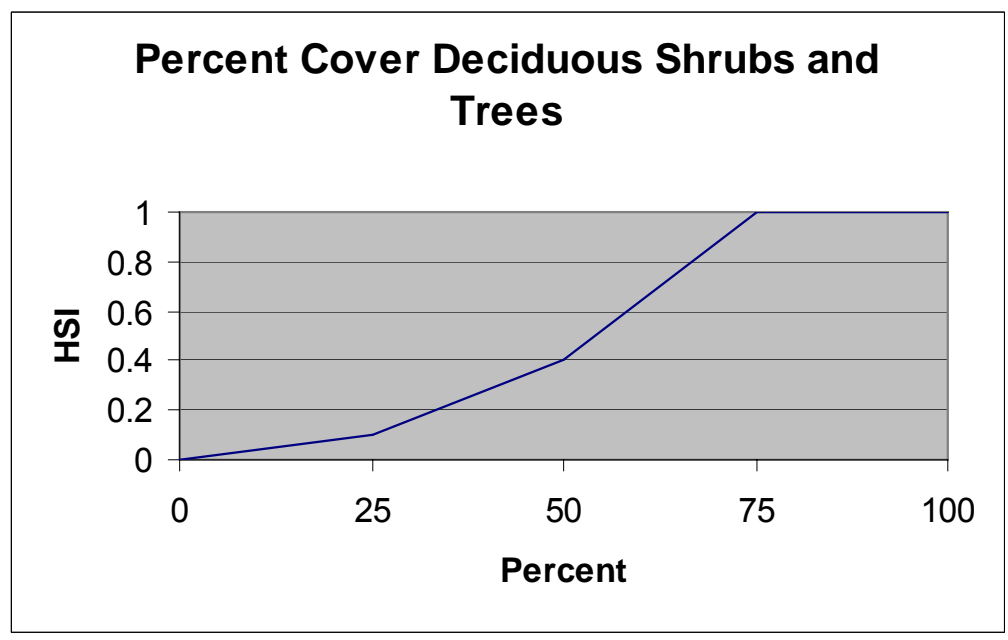

V9: Deciduous Shrub and Tree Composition/Wheat Availability

\begin{tabular}{|c|c|c|}
\hline Attribute & Species & SI \\
\hline Upper Canopy & Water Birch, Aspen, Cottonwood & 0.5 \\
\hline Mid Canopy & $\begin{array}{c}\text { Serviceberry, Hawthorn, } \\
\text { Chokecherry }\end{array}$ & 0.3 \\
\hline Lower canopy & Rose, Snowberry & 0.2 \\
\hline $\begin{array}{c}\text { Agricultural } \\
\text { Fields }\end{array}$ & Standing Wheat or Wheat Stubble & 0.2 \\
\hline HSI & Additive : Not to exceed 1.0 & $1.2=$ \\
& & 1.0 \\
\hline
\end{tabular}


West Foster Creek Expansion Project

V10: Percent Area Providing Winter Habitat

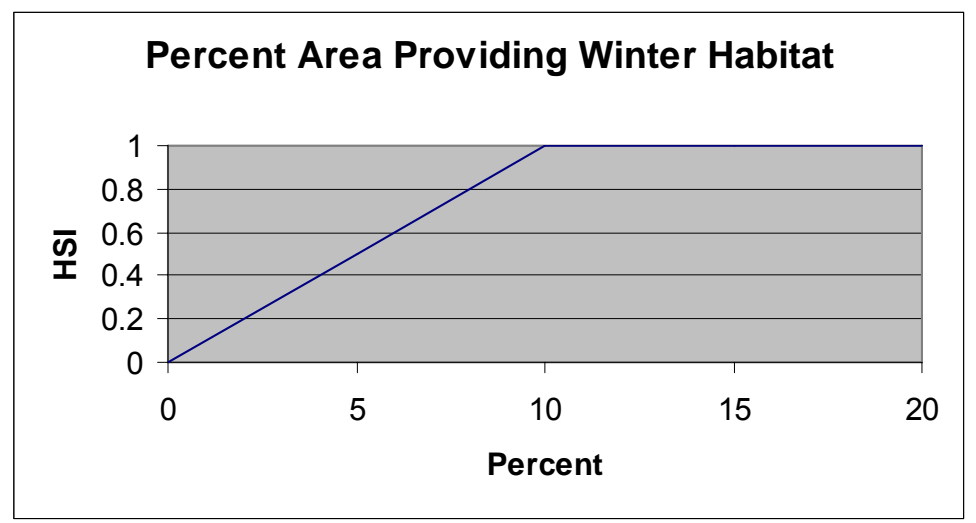

Winter HSI Equation: $\left((\mathrm{V} 8 \times \mathrm{V9})^{1 / 2} \times \mathrm{V10}\right)$

Model HSI: Consists of two HSI's: Nesting/Brood Rearing HSI and Winter HSI.

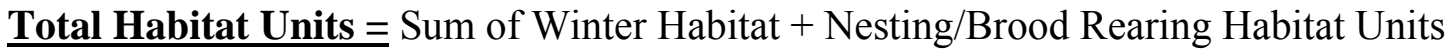




\section{Western Meadowlark}

\section{WESTERN MEADOWLARK}

Modified from Schroeder and Sousa, 1982.

Cover Types: Grassland, Shrubgrass, Shrubland, Pasture, Shrub-steppe

V1: Percent canopy cover of herbaceous plants

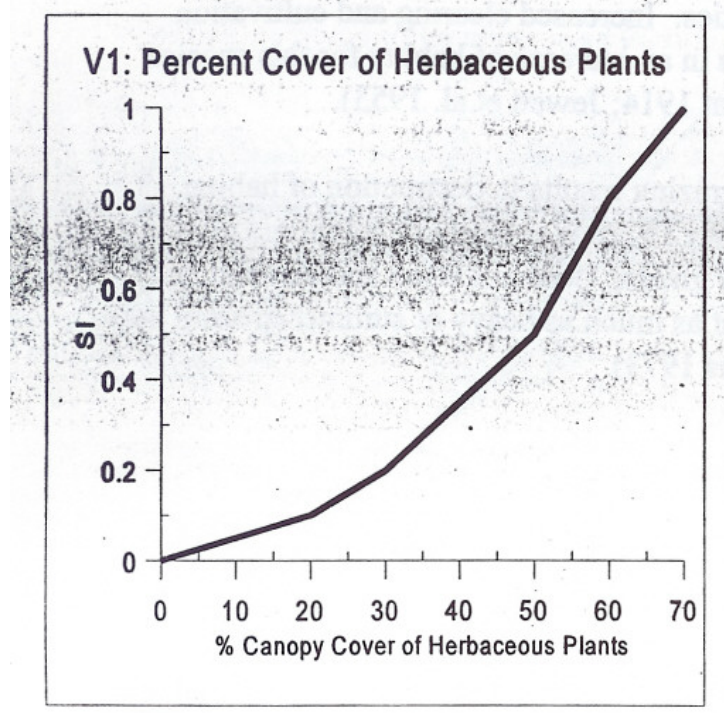

\section{Cover Requirements}

Western meadowlarks are adapted to short grass and mixed grass prairies, preferring large fields with short vegetation and good drainage. Western meadowlarks exhibit tolerance for a wide variety of plant associations and are widely distributed in Washington-commonly occurring in meadows, orchards, thickets, and cultivated areas. Conversion of woodlands to agricultural fields has favored western meadowlark populations in Washington.

V2: Percent of herbaceous canopy cover composed of grass

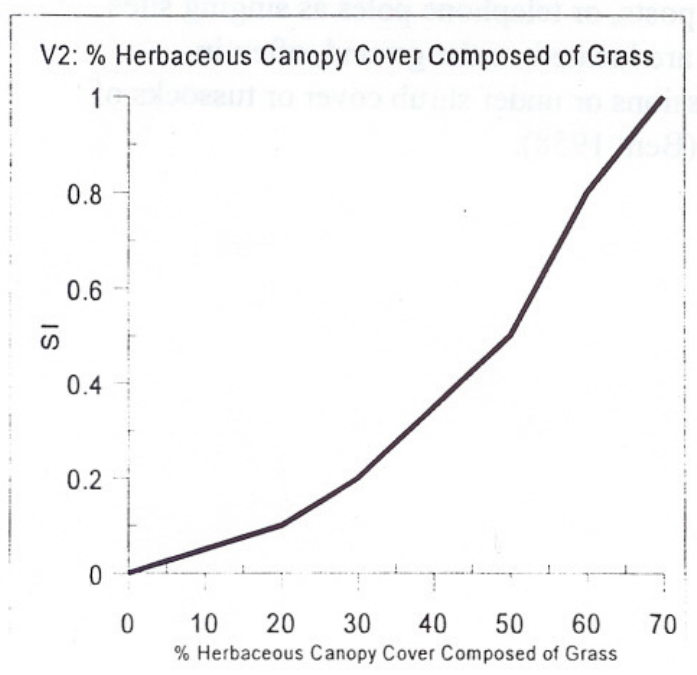

\section{Food Requirements}

Bryant (1914), Weins (1973) et.al. suggest that animal material, primarily insects, comprise approximately $63 \%$ of the meadowlark's diet while $37 \%$ is made up of vegetative matter. Vegetable matter consisted of one-third grain and two-thirds weed seeds. Spring and summer diet was primarily insects with a shift to seeds in fall and winter. Hubbard and Hubbard (1969) reported meadowlarks eating carrion including their own species. It is doubtful that food supply is ever a limiting factor for this species (Lanyon, 1956).

Water Reauirements 


\section{West Foster Creek Expansion Project}

V3: Average height of herbaceous canopy (inches)

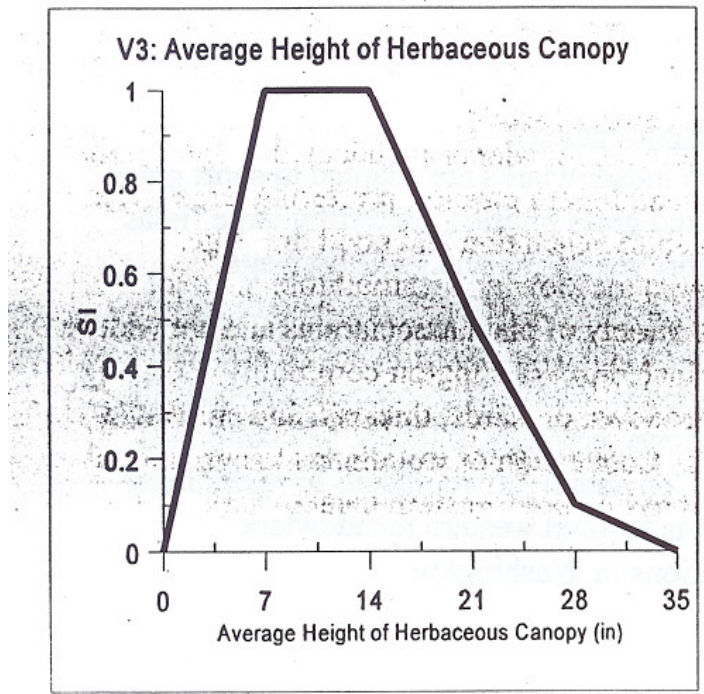

V4: Distance to Perch Sites (feet)

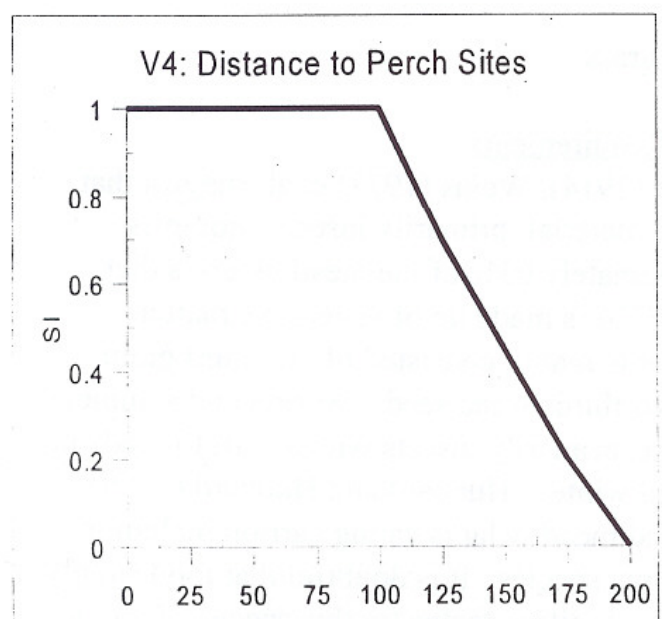

Because of its habitat preferences, western meadowlarks are affected by agricultural activities. Increased clearing and cultivation results in an increase of habitat for this species (Bryant 1914; Jewett et.al. 1953).

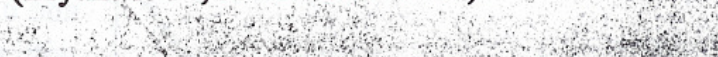

Overgrazing results in destruction of habitat (Rohwer 1972. Weins 1973). Light grazing or winter grazing does not affect meadowlark habitat as much as heavy or summer grazing (Weins 1973)

Reproductive Requirements Males require elevated perches, such as shrubs, fence posts, or telephone poles as singing sites. Nests are located on the ground, often in depressions or under shrub cover or tussocks of grass (Bent 1958). 
West Foster Creek Expansion Project

V5: Percent Shrub Canopy Cover

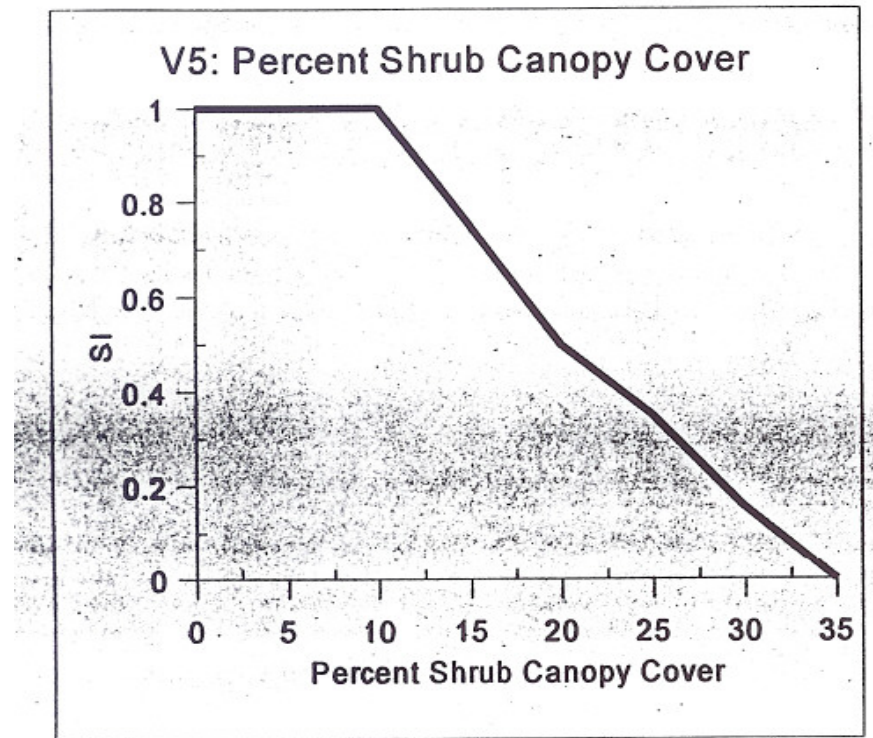

Model Equation:

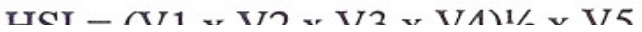

$\mathrm{HSI}=(\mathrm{V} 1 \times \mathrm{V} 2 \times \mathrm{V} 3 \times \mathrm{V} 4)^{1 / 2} \times \mathrm{V} 5$ 


\section{West Foster Creek Expansion Project}

\section{Bobcat}

Variable 4. Percent of area comprised of rockpiles, rock outcrops, rocky ledges, boulder fields, talus slopes and cliffs [include only tops and bottoms of cliffs and not cliff faces (pers comm., Steve Knick)].

Assumes:

(1) Bobcats prefer rocky or broken terrain.

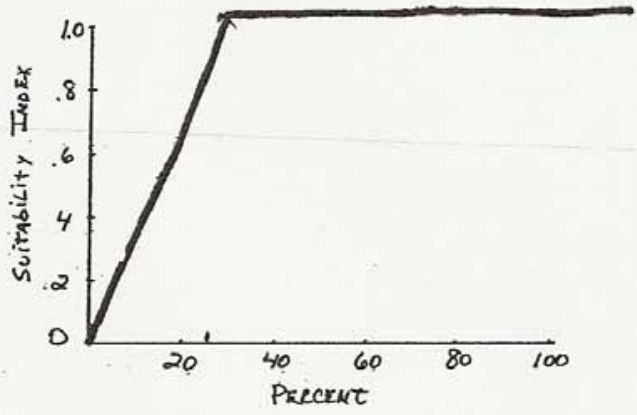

Model Relationships

In order to calculate suitability indices for food and for cover, the variables for each life requisite were combined into an equation. Because food requirements and cover/reproductive requirements are of equal importance, the SI's were derived to express each life requisite as separate values for the overall HSI determination (see below).

Suitability Indices

Food

$$
\mathrm{SI}_{f}=\frac{\mathrm{V} 1+2 \mathrm{~V} 2}{3}
$$

Cover/reproduction

$$
\mathrm{SI}_{\mathrm{c} / \mathrm{r}}=\frac{\mathrm{V} 3+2 \mathrm{~V} 4}{3}
$$


West Foster Creek Expansion Project

\section{Mink}

$\begin{aligned} & \text { Cover } \\ & \text { type }\end{aligned}$
EFW,DFW,

Percent tree, shrub, and/or persistent emergent herbaceous vegetation canopy closure.

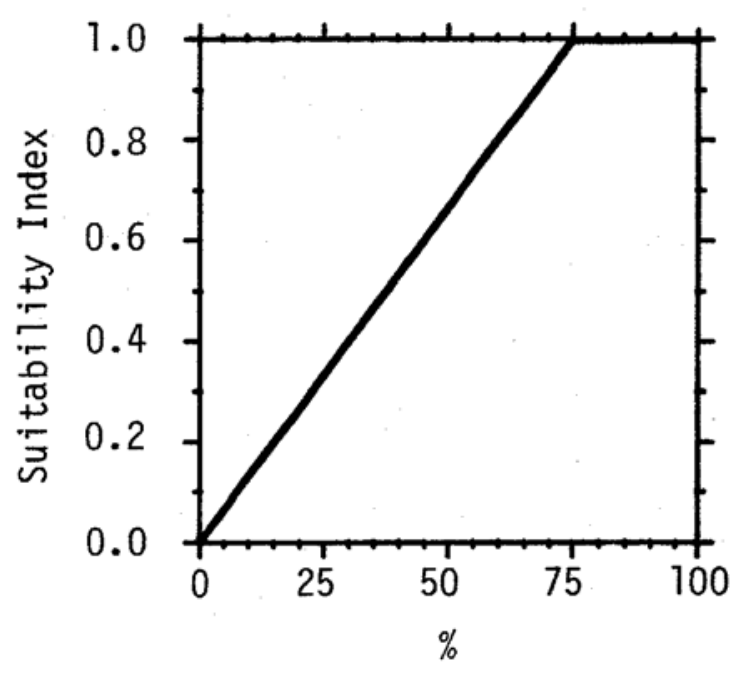


West Foster Creek Expansion Project

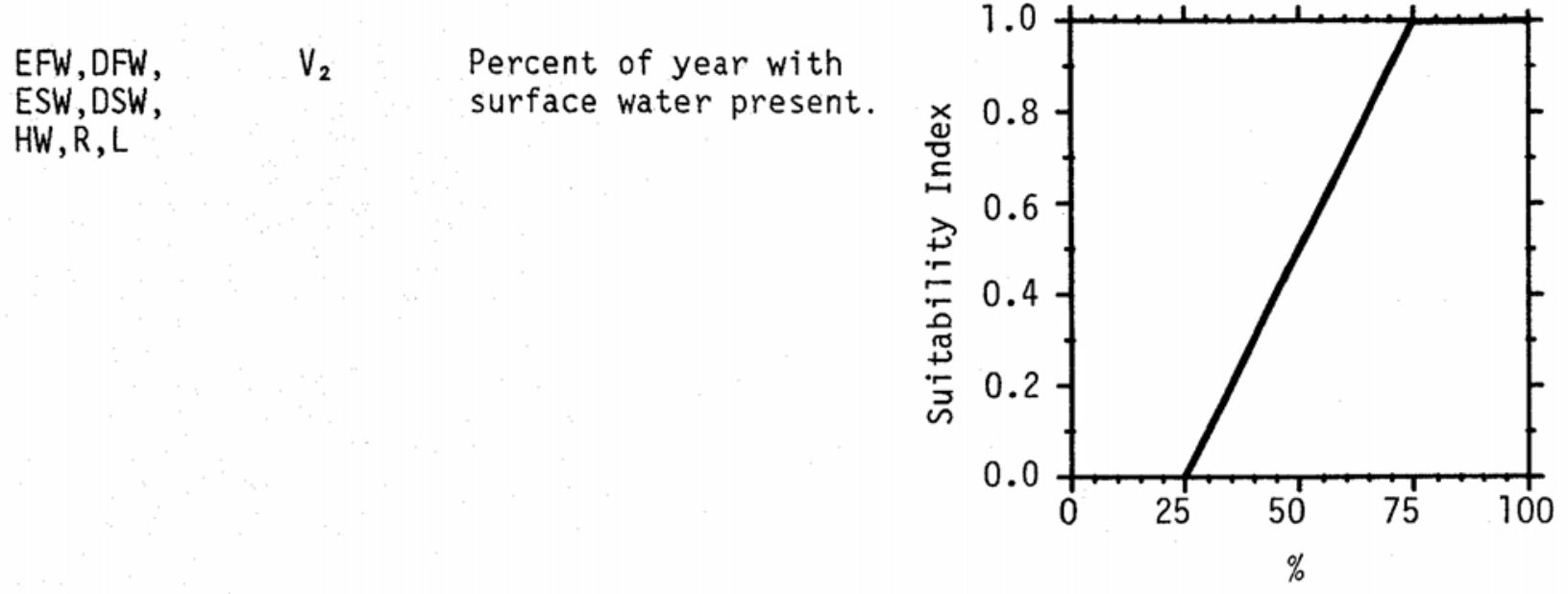

HW

$V_{3}$

Percent of wetland basin dominated by persistent emergent herbaceous vegetation.

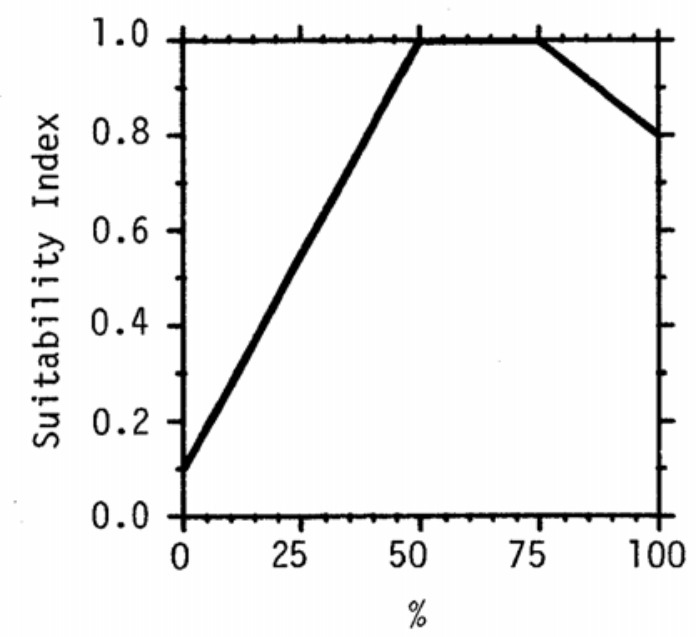

EFW, DFW,

$V_{4}$

Percent tree and/or shrub canopy closure within $100 \mathrm{~m}$ (328 ft) of water's or wetland's edge.

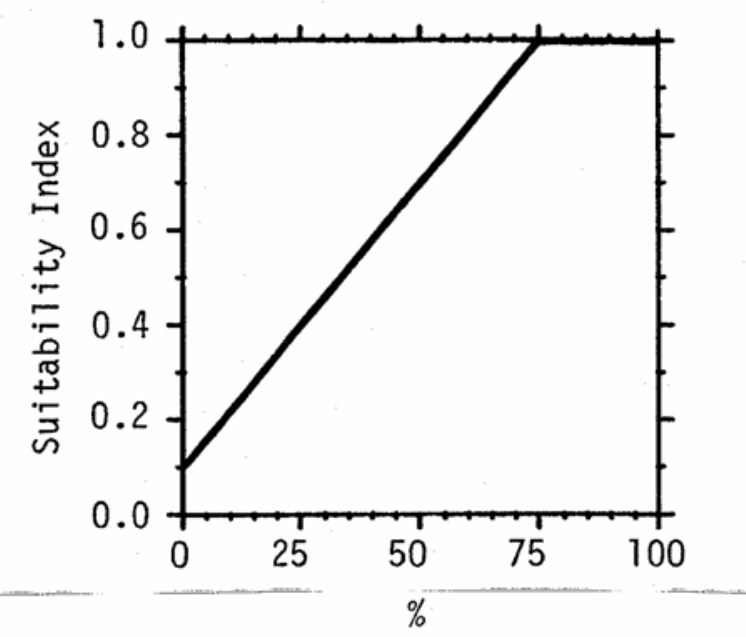


West Foster Creek Expansion Project

$\mathrm{L}$

$$
\begin{array}{ll}
V_{5} & \begin{array}{l}
\text { Shorel ine devel- } \\
\text { opment factor. }
\end{array}
\end{array}
$$

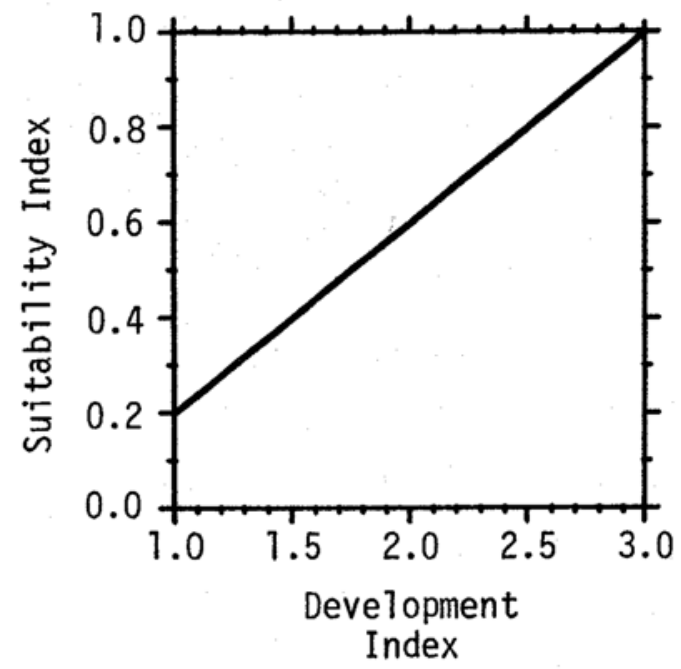

Equations. In order to obtain life requisite values for the mink, the SI values for appropriate variables must be combined through the use of equations. A discussion and explanation of the assumed relationships between variables was included under Model Description, and the specific equations in this model were chosen to mimic these perceived biological relationships as closely as possible. The suggested equations for obtaining a food/cover value are presented by cover type in Figure 2.

Life requisite

Food/cover

Food/cover

Food/cover

Food/cover

Food/cover
Cover type

EFW,DFW, ESW, DSW

$[<405$ ha $(1,000$ acres $)$ in size]

EFW, DFW, ESW, DSW

$[\geq 405$ ha $(1,000$ acres $)$ in size]

\section{Equations}

$V_{2} \frac{V_{1}+V_{4}}{2}$

$\left(V_{1} \times V_{2}\right)^{1 / 2}$

HW

$V_{2} \frac{4 V_{3}+V_{4}}{5}$

$\left(V_{4} \times V_{5}\right)^{1 / 2}$

R
$\left(V_{2}{ }^{2} \times V_{4}\right)^{1 / 3}$ 


\section{Application of the Model}

Potential mink habitat must contain a relatively permanent source of surface water. Because of the mink's use of upland habitats for denning and foraging, optimum mink habitat must also contain suitable cover adjacent to the water body or wetland. Therefore, the application of this model and the determination of habitat units is based on an evaluation of the quality of the wetland, lacustrine, or riverine cover type and a $100 \mathrm{~m}(328 \mathrm{ft}$ ) band of habitat surrounding the aquatic portion of the habitat. Figure 3 illustrates the relationship of cover types to the suggested evaluation area.

\section{Cover type}

Lacustrine

HSI determined only for area contained within $100 \mathrm{~m}$ (328 ft) band around lake.

Riverine

HSI determined for area within $100 \mathrm{~m}$ band on both sides of river plus area of river.

Palustrine (herbaceous wetlands, forested wetlands, or shrub wetlands). Less than 405 ha ( 1,000 acres) in size.

HSI determined for area contained within cover type plus area within $100 \mathrm{~m}$ band around wetland cover type.

Palustrine (forested wetlands or shrub wetlands) 405 ha $(1,000$ acres) or larger in size HSI determined for area contained only within cover type.

\section{Area for evaluation}
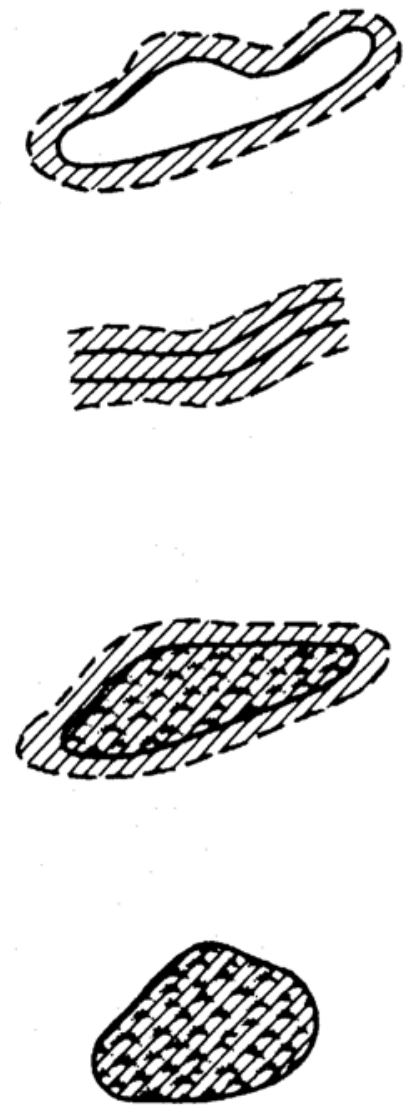

Figure 3. Guidelines for determining the area to be evaluated for mink habitat suitability under various cover type conditions. 


\section{Black-capped Chickadee}

\section{Model Relationships}

Suitability Index (SI) graphs for habitat variables. This section contains SI graphs that illustrate the habitat relationships described in the previous section.

Cover

type

$\begin{array}{cl}\frac{\text { Variable }}{V_{1}} & \begin{array}{l}\text { Percent tree } \\ \text { canopy closure. }\end{array}\end{array}$

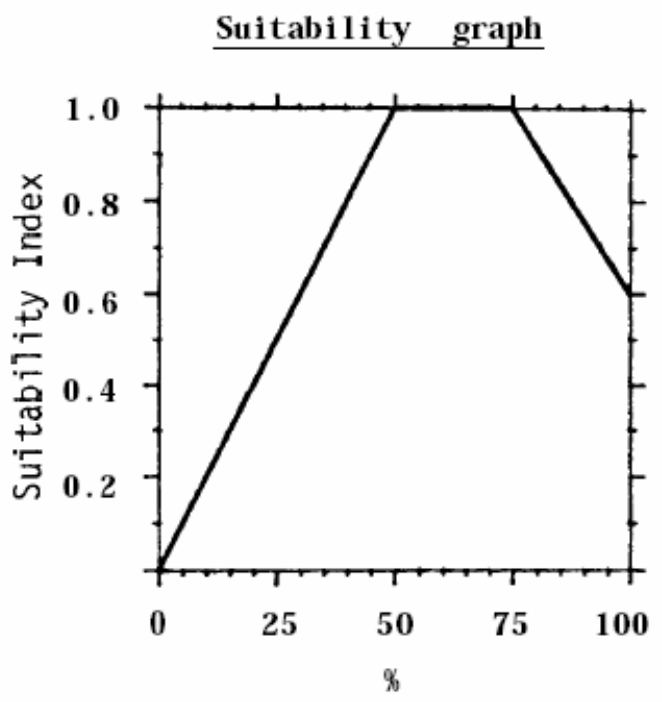

DF, EF,

DFW, EFW

$V_{2} \quad$ Average height of
overstory trees.

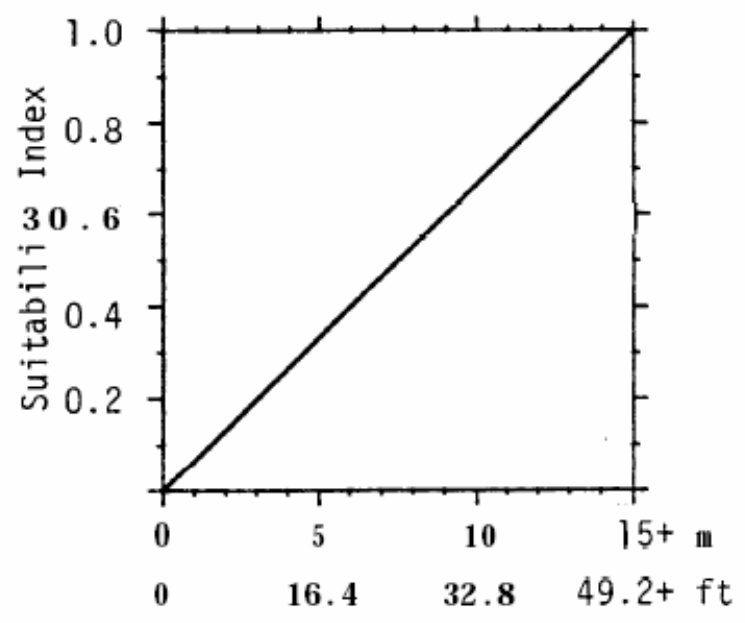


West Foster Creek Expansion Project

DF , EF,

DFW, EFW
$V_{3}$

3

Tree canopy volume/ area of ground surface.
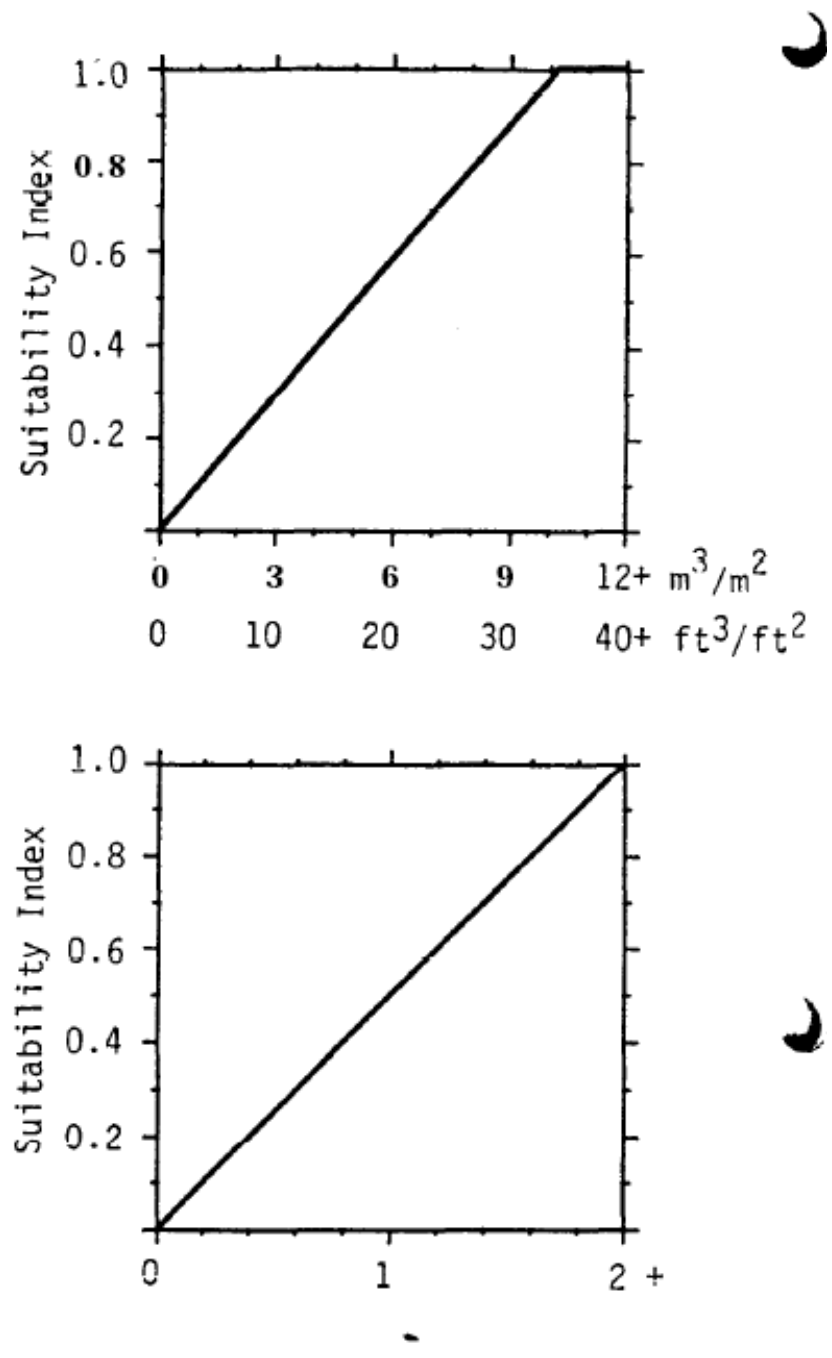

Equations. In order to determine life requisite values for the black-

DF , EF, D F W , E F W

\begin{abstract}
$V_{4}$
\end{abstract}

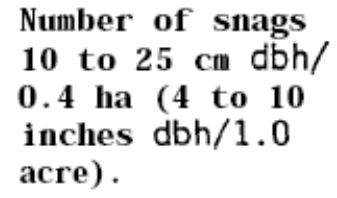
capped chickadee, the SI values for appropriate variables must be combined through the use of equations. A discussion and explanation of the assumed relationships between variables was included under Model Description, and the specific equations in this model were chosen to mimic these perceived biological relationships as closely as possible. The suggested equations for obtaining food and reproduction values are presented below. 
West Foster Creek Expansion Project

\section{Life requisite}

Food

Reproduction
Cover type

DF , EF , DFW , EFW

DF , EF , DFW , EFW
Equation

$$
\begin{aligned}
& \left(V_{1} \times V_{2}\right)^{1 / 2} \text { or } V_{3} \text { (See page } \\
& 5 \text { for discussion on which } \\
& \text { to use) } \\
& V_{4}
\end{aligned}
$$

HSI determination. The HSI for the black-capped chickadee is equal to the lowest life requisite value.

Application of the Model

Definitions of variables and suggested field measurement techniques (from Hays et al. 1981, unless otherwise noted) are provided in Figure 3 . 


\section{Mallard}

COLUMBIA BASIN WILDLIFE AREA MALLARD HEP MODEL

(WP:CBWMAL- Revised 10 Feb 99 and November 2007)

This model was developed from information provided in several different models including:

(I)the Draft Habitat Suitability Index model, Mallard (Breeding), US Fish and Wildlife Service, Division of Ecological Services, Sacramento, California, July, 1985; (2) Draft Habitat Suitability Index Model, Mallard (Wintering), US Fish and IVildlife Service, Division of Ecological

Services, Sacramento, California, July, 1985; and (3) Habitat Suitability Index Models:

Dabbling Ducks, by Patricia D. Rice, US Fish and Wildlife Service, Great Basin Complex, Reno, Nevada, February, 1984. These models were modified for the Dalles, John Day, and McNary wildlife loss assessment by HEP team members according to information provided by the local, state, federal, and tribal biologists.

This model was further modified by Columbia River Wildlife Mitigation Team biologists in 1998 to account for local conditions, impacts of carp (Cyprinus carpio) on mallard brood rearing habitat quality in the Columbia Basin, and new research. Modifications to the original loss assessment mallard model are accompanied by text and cited if possible (modifications compiled

by Paul R Ashley, Senior Wildlife Mitigation Biologist WDFW).

\section{Mallard Brood Rearing}

Cover Types: Emergent wetland, Lacustrine, Palustrine

\section{V7: Percent emergent cover to percent open water ratio}

$\begin{array}{lcccccc}\text { \% Cover:Water Ratio } & 0: 100 & 20: 80 & 40: 60 & 60: 40 & 80: 20 & 100: 0 \\ \text { SI } & 0.2 & 0.4 & 1 & 1 & 0.4 & 0.2\end{array}$

\section{V8: Water Permanence}

1. Permanently flooded

SI

2. Intermittently exposed

3. Semi-permanently flooded

4. Seasonally flooded

5. Temporarily flooded

6. Intermittently flooded

The presence of surface water within a wetland significantly influences mallard reproductive habitat quality. Wetlands that do not maintain surface water throughout the breeding season (April through June, Bellrose 1978) are unsuitable reproductive habitat. Intermittently flooded (6) and temporarily flooded (5) wetlands typically have surface water for a short period during the breeding season, or are flooded pre/post breeding season resulting in unsuitable reproductive habitat. Semi-permanently flooded (4) wetlands contain surface water throughout the 


\section{West Foster Creek Expansion Project}

growing/nesting season and are assumed to be optimum breeding habitat.

Depending on the abundance of vegetation and duration of surface water present, seasonally flooded (3) wetlands may have some reproductive potential for mallards. The value assigned to seasonally flooded wetlands is relatively low due to the limited presence of surface water in all years.

Likewise, intermittently exposed (2) wetlands represent slightly less than optimum reproductive habitat based on the absence of surface water within these wetlands during unusually low precipitation years. Excessive depth and typical large size may render permanently flooded (1) wetlands (lacustrine habitat types) less desirable reproductive habitat due to limited emergent/submergent vegetation, low nutrient content, and low invertebrate availability.

SI $=$ Absent: 1.0

Present: 0.5 (Riverine cover types, streams, flowing water)

Present: 0.1 (Lacustrine/palustrine cover types, emergent wetlands, impoundments, etc.)

Carp decrease water quality for waterfowl and other species by consuming aquatic vegetation and increasing turbidity resulting in production of blue/green algae that displaces valuable aquatic flora and decreases habitat quality for aquatic invertebrates. After consuming emergent/submergent vegetation, carp will compete directly with waterfowl broods for reduced numbers of aquatic organisms. Hames (1998) reports that carp do not impact water quality for mallards in flowing rivers, streams, and canals to the extent that non-flowing/slack water habitats are impacted, because sediments from carp activities are removed from the immediate area by moving water.

V9 was added to model as suggested by Robert Kent, Jim Tabor, and R. Duff (pers. comm., WDFW, 1998). 


\section{Appendix B - Measurement Protocols}

\section{HABITAT EVALUATION PROCEDURES}

STANDARD MEASUREMENT PROTOCOLS AND TECHNIQUES (Draft)

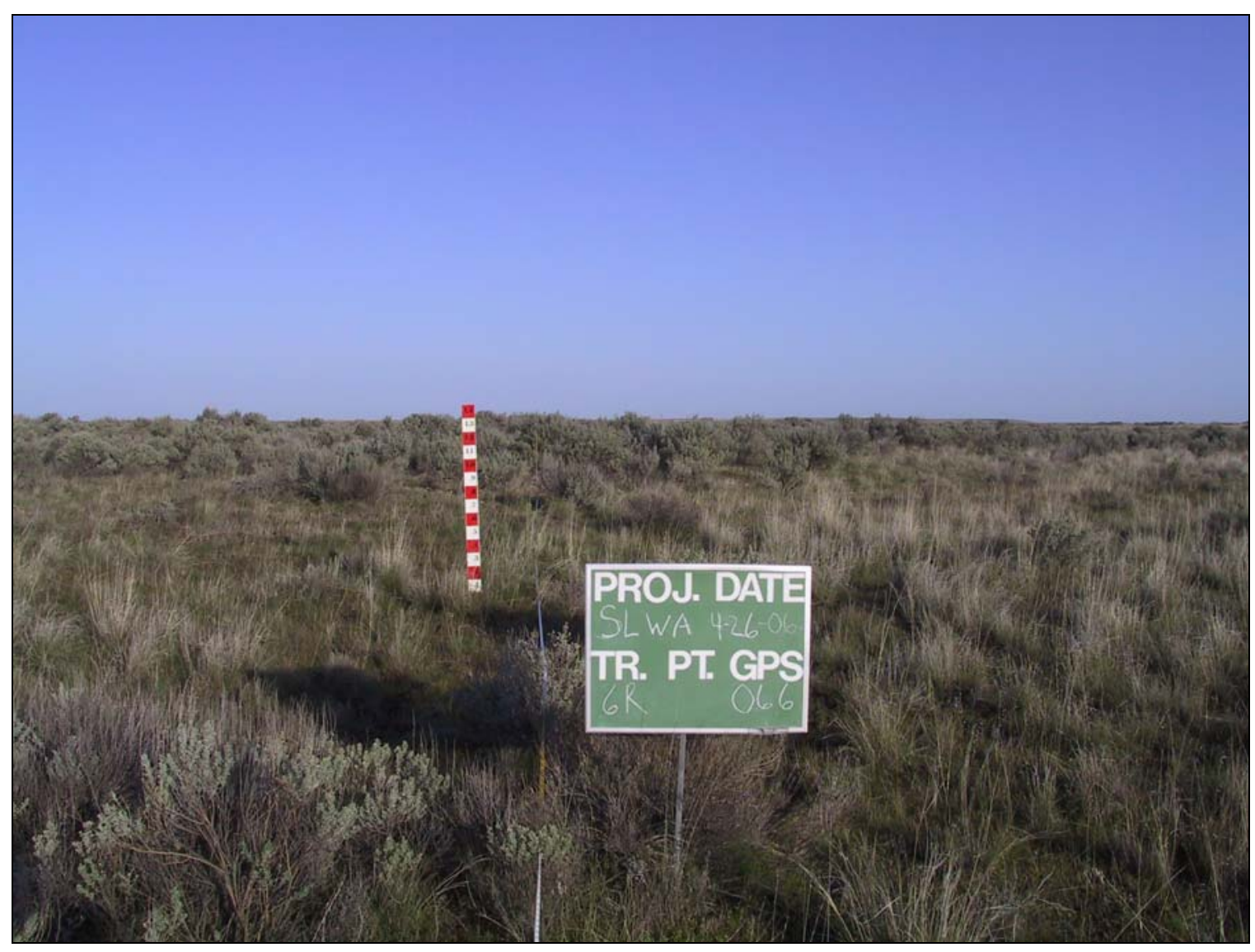

Compiled By

Paul R Ashley - RHT Coordinator

November 2006 


\section{HEP Sampling Design and Measurement Protocols}

\section{Introduction}

This document was developed to fulfill a request by the Upper Columbia United Tribes (UCUT) and Bonneville Power Administration (BPA) to develop a "stand alone" reference for Habitat Evaluation Procedures (HEP) transect protocols used by the Regional HEP Team (RHT). General and specific protocols are described. General protocols include a brief description of pre HEP survey pilot studies; transect establishment guidelines, and photo documentation parameters. In contrast, specific metrics detail actual habitat variable measurement techniques including diagrams where additional explanation is needed.

Specific metrics are identified with an alpha-numeric code. This allows project managers and others to identify specific measurement techniques in report tables without lengthy, redundant explanations. This report is intended to be a "living" document and will be modified as needed. The following standardized protocols and measurement techniques are used by the Regional HEP team to measure habitat variables described in HEP models.

\section{General Protocols}

\section{Pilot Studies}

Pilot studies are conducted in new habitat types and/or familiar habitat types that are comprised of unique structural conditions/key ecological correlates. Pilot study data is used to estimate the sample size needed for a confidence level $\geq 80 \%$ with a $10 \%$ tolerable error level (Avery 1994) and to determine the most appropriate sampling unit ${ }^{13}$ for the habitat variable of interest i.e., a coefficient of variation analysis (BLM 1998). In addition, a power analysis is conducted on pilot study data (and periodically throughout data collection) to ensure that sample sizes are sufficient to identify a minimal detectable change of $20 \%$ in the variable of interest with a Type I error rate $\leq 0.10$ and $\mathrm{P}=0.9$ (BLM 1998, Block et al. 2001). All field data is recorded on data loggers or data sheets and downloaded/transferred to data summary spreadsheets.

Transects

Transect cover sheets are used to document specific transect information including transect identification, cover type, HEP Team members, global positioning system (GPS) coordinates, and other pertinent information.

Transects are established at least 300 feet (100 meters), where possible, from ecotones, roads, and other anthropogenic influences. Transect starting points and azimuths (direction) are randomly selected for each cover type. Start points are selected based on superimposing a UTM grid over cover type maps and identifying specific X/Y coordinates with the aid of a random numbers table, or computer generated random number generator/point locater program.

\footnotetext{
${ }^{13}$ Includes micro-plot grid size and shape etc.
} 
Transect start, turn, and end points are marked with 14 -inch (36 centimeter) 0.25 inch ( 0.6 centimeter) diameter rebar stakes ${ }^{14}$ painted fluorescent orange or red. GPS positions (UTM coordinates-NAD 27) are recorded at start, turn, and end points. If cover types change or transect length is greater than 300 feet, another transect azimuth is randomly selected, or the original azimuth is varied by 45 degrees (direction [left or right] is determined by the flip of a coin where more than one choice is possible). Compass azimuths (headings) are magnetic bearings i.e., not corrected for local declination. Transects are divided into 100 foot (30 meter) sample units for statistical purposes.

\section{Photo Points}

Photo points are established at the start point of each transect. Pictures are recorded from a height of three feet at the beginning of each transect while facing in the direction of the transect azimuth. A transect reference board (includes transect number, project name, date, GPS reference number) is placed at the 15 foot interval while a cover board is placed at the 30 foot mark on each transect. Occasionally, panoramic photographs are also needed e.g., dense vegetation, linear/narrow cover types. Habitat conditions are photographed with a Canon G1 ${ }^{\circledR} 3.3$ mega pixal digital camera (with and without magnification).

\section{Specific Metrics}

Metrics generally follow those described by Hays et al. (1981) and/or Avery (1994) unless otherwise noted. Some metrics have been modified due to extreme field conditions and/or to better meet Regional HEP Team needs.

\section{Herbaceous Measurements}

\section{Percent Cover}

1. Herbaceous percent cover measurements are recorded at 20 or 25 -foot intervals on the right side of the transect tape (the right side is determined by standing at 0 feet and facing the line of travel/transect azimuth). RHT members walk on the left side of the transect line to reduce sample disturbance. A square $0.1 \mathrm{~m}^{2}$ micro-plot grid is used in grasslands to estimate percent cover of herbaceous vegetation while a rectangular $0.5 \mathrm{~m}^{2}$ grid is generally used in shrublands (the $0.5 \mathrm{~m}^{2}$ grid may also be used in grasslands if desired). The near right hand corner of the grid is placed at the sampling interval (rectangle grids are placed with the long axis perpendicular to the tape, and the lower right corner on the sampling interval). An example of micro-plot grid placement is shown in Figure 1. Approximately $20 \%$ of the micro plot is covered by vegetation in the example. Grid samples are considered independent samples for statistical purposes.

1A: $0.1 \mathrm{~m}^{2}$ micro-plot grid/20' interval

\footnotetext{
${ }^{14}$ Marking transect points with rebar stakes is at the discretion of the project proponent. Therefore, not all transects are marked in this manner.
} 
1B: $0.1 \mathrm{~m}^{2}$ micro-plot grid $/ 25$ interval

1C: $0.5 \mathrm{~m}^{2}$ micro-plot grid/20' interval

1D: $0.5 \mathrm{~m}^{2}$ micro-plot grid $/ 25$ ' interval

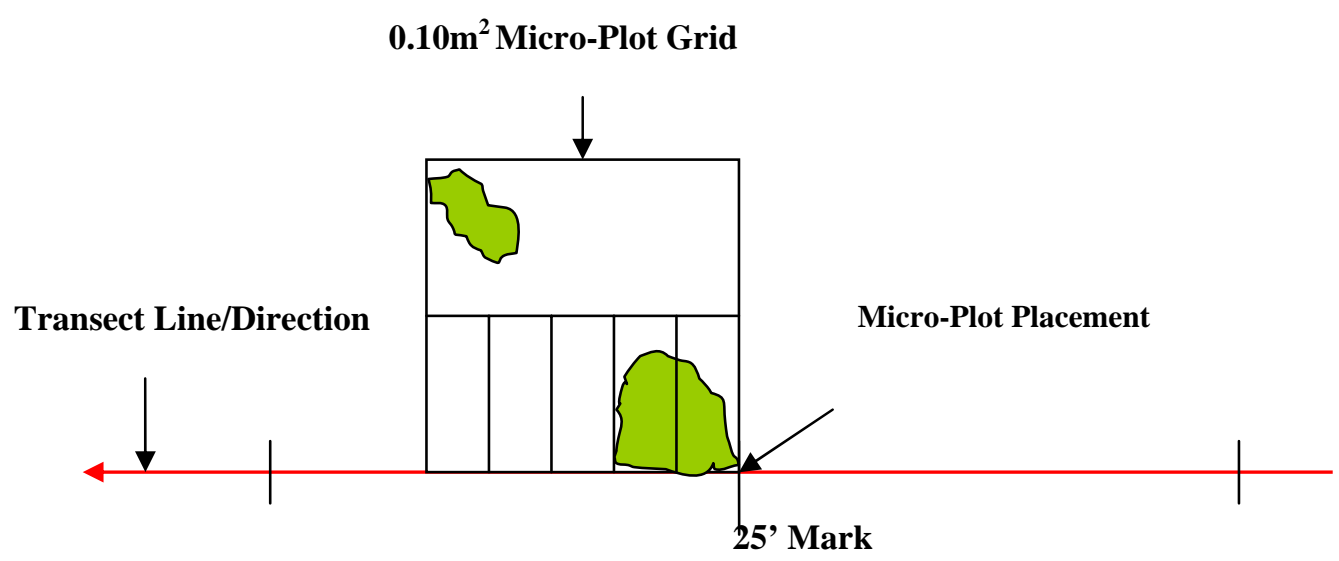

Figure 1. Micro-plot grid placement and percent cover example.

\section{Height}

2. Herbaceous height is measured with a measuring rod placed within the grid frame (scale $=10$ ths $/ \mathrm{ft}$.). Three evenly spaced measurements are recorded and averaged for each sample. Only leaf material is measured (leaves provide the greatest amount of cover). "Leaf material" may include residual cover and/or new growth predicated on HEP model variable requirements. Grass inflorescence is not included in height measurements.

2A. Four measurements, one from each corner of the micro plot grid, are recorded and averaged for each sample. Only leaf material is measured (leaves provide the greatest amount of cover). Grass inflorescence is not included in height measurements.

2B. A measuring rod is held vertical at the interval point: the highest vegetation to cross the measuring rod at that point is measured to the nearest tenth of a foot.

2B-1: 10' interval

2B-2: 20 ' interval

2B-3: 25 ' interval

\section{Visual Obstruction Readings (VOR)}


3. A Robel pole (Robel 1975) is used to document vertical and/or horizontal cover for herbaceous vegetation i.e., visual obstruction readings (VOR). Measurements are recorded at 20,25, or 50-foot intervals. Intervals are determined by the length of each transect, i.e., a minimum of 12 measurements are required for each transect, or cover type heterogeneity (structurally diverse cover types generally require larger sample sizes).

The Robel pole (Robel 1975) is placed on the transect line at the appropriate interval. Four observations are taken from a distance of four meters from the Robel pole and averaged to obtain a single visual obstruction reading or VOR. Observers sight over a one meter pole and record how much of the Robel pole is totally obscured from the ground up (Figure 2). Measurements are reported in 0.25 decimeter increments.

Two measurements are taken on the transect line on opposite sides of the Robel pole; two identical measurements are taken from the same point perpendicular to the transect line for a total of four "readings" (Figure 3). Sample size is determined to be adequate when the "running mean" varies $\leq 10 \%$ of the mean. VOR samples are considered independent for statistical purposes.

3A: 20 ' interval

3B: 25 ' interval

$3 \mathrm{C}$ : 50 ' interval

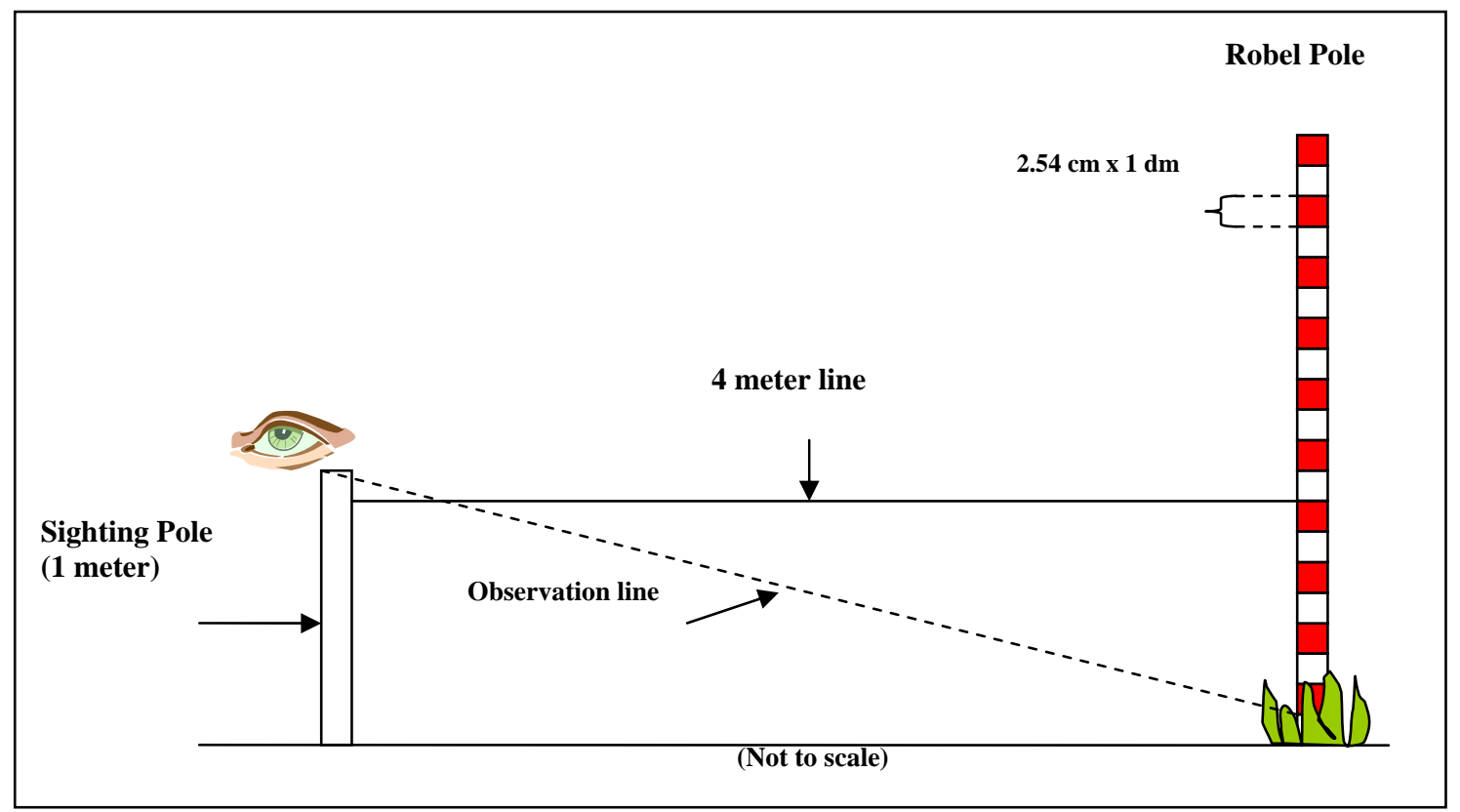

Figure 2. Visual obstruction reading diagram. 


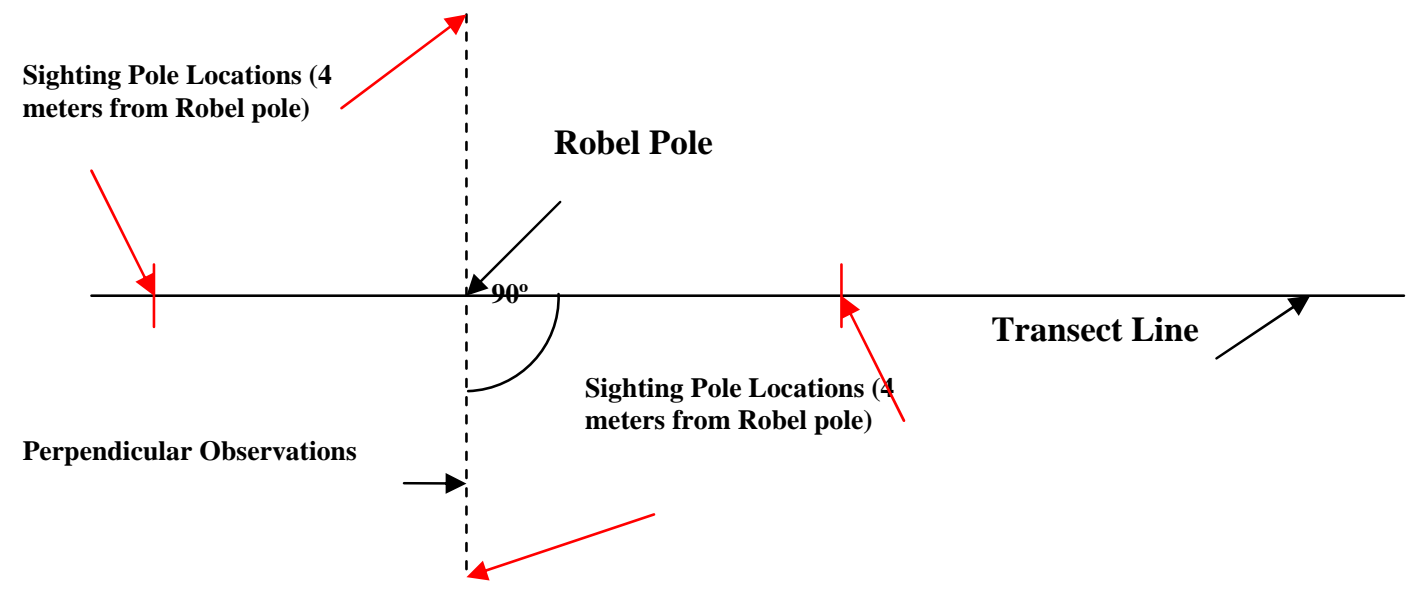

(“Birds eye” View)

Figure 3. Robel pole "readings" layout diagram.

\section{Shrub Measurements}

\section{Percent Cover}

4. Line intercept or point intercept (USFWS 1981) is used to determine shrub cover. Line intercept is generally used when shrub cover is estimated at $<5 \%$ (the most accurate results are obtained using the line intercept method). In contrast, the point intercept method is used if shrub cover is estimated at $>5 \%$.

4A: Line intercept is used to measure the amount of cover that intercepts the transect line as illustrated by the red lines shown in Figure 4. Measurements are in $10^{\text {ths }}$ of feet. Gaps in vegetation less than four tenths of a foot (5 inches) are ignored. The amount covered by shrubs is added to determine shrub intercept for each transect. For example, if 7.5 feet of a 100 -foot long transect is covered by shrubs, percent cover is $7.5 \%$.

Shrub cover is recorded by species. Where shrubs overlap, shrub intercept is recorded for the tallest shrub and noted for the lower shrub(s). 


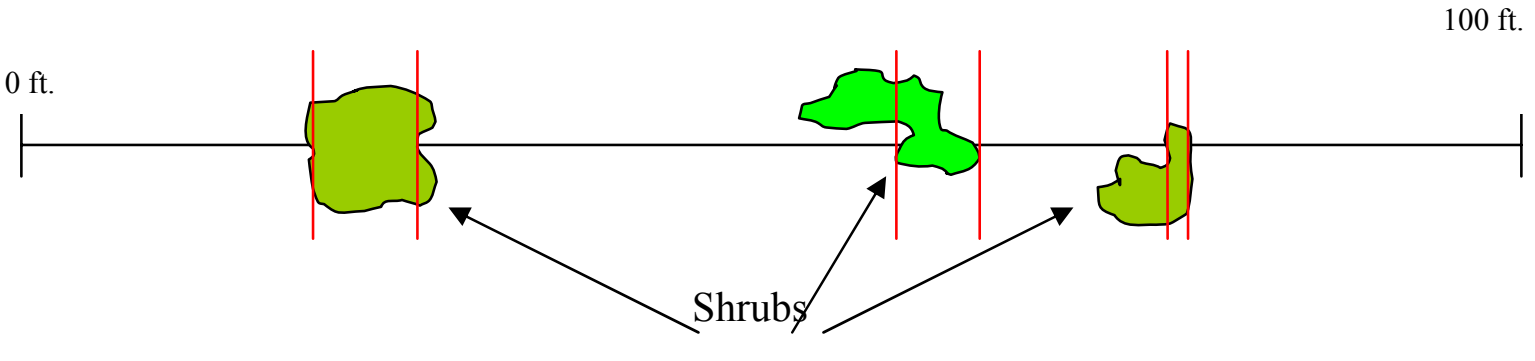

Figure 4. Line intercept method example.

4B: Point intercept is used when shrub canopy cover is estimated at $\geq 5 \%$.

Shrub cover is determined by recording the number of "hits" at specific intervals along a transect line. To be counted as a "hit", a portion of the shrub must cross the transect tape's interval number line e.g., 2', 4', 6'... nth. If a portion of the shrub does not break the vertical plane at the interval number line, it is reported as a miss (Figure 5). Either a "hit" or "miss" is recorded on data loggers and/or paper data sheets for each designated interval.

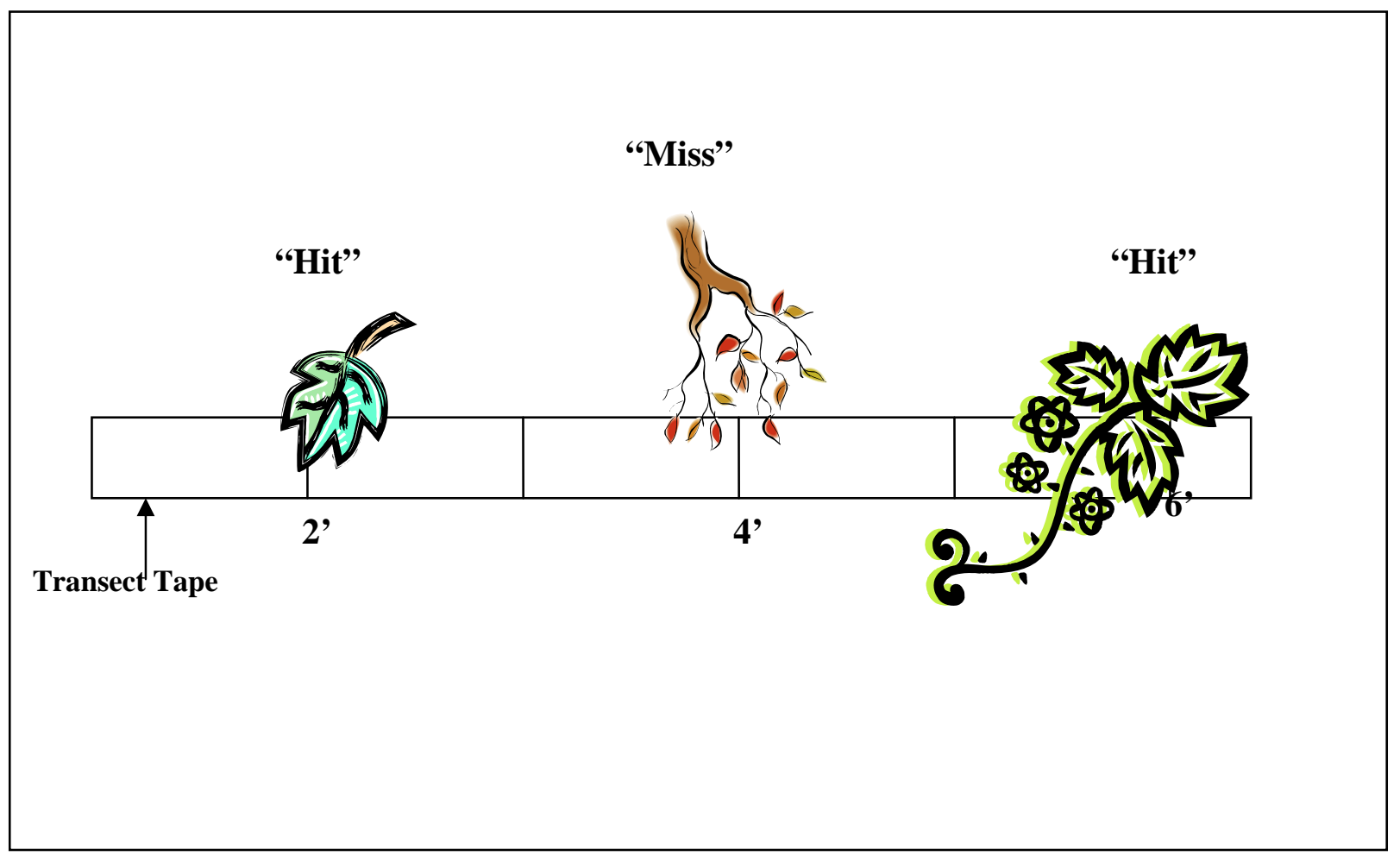

Figure 5. Point intercept method example showing "hits" and "misses" at two foot intervals. 
From $5 \%$ to $20 \%$ cover, point data is collected at two-foot intervals ( 50 possible "hits" per $100 \mathrm{ft}$. sample unit). If shrub cover is estimated at $>20 \%$, shrub point data is collected at five foot intervals (20 possible "hits" per 100 $\mathrm{ft}$. sample unit). On rare occasions, ten-foot intervals may be used when shrub cover exceeds 50\% (10 possible "hits" per $100 \mathrm{ft}$. sample unit). The ten-foot interval is generally applied to shrub monocultures, or areas with few shrub species that exhibit relatively equal shrub distribution/density.

Shrub "hits" are recorded by species. Where shrubs overlap, shrub intercept is recorded for the tallest shrub and noted for the lower shrub(s).

4B-1: 2' interval

4B-2: 5 ' interval

4B-3: 10' interval

4C: Modified point method is used when shrub cover is impenetrable or otherwise inaccessible. A baseline transect is established along the shrub edge. A six-foot measuring rod is then inserted into the shrub cover at right angles to the baseline tape at appropriate intervals. Recorders estimate shrub "hits", species information, and height data where the end of the six-foot measuring rod intercepts the shrub cover (Figure 6). As with point intercept, intervals may very. Shrubs are identified by species.

4C-1: 2' interval

4C-2: 5 ' interval

4C-3: 10' interval

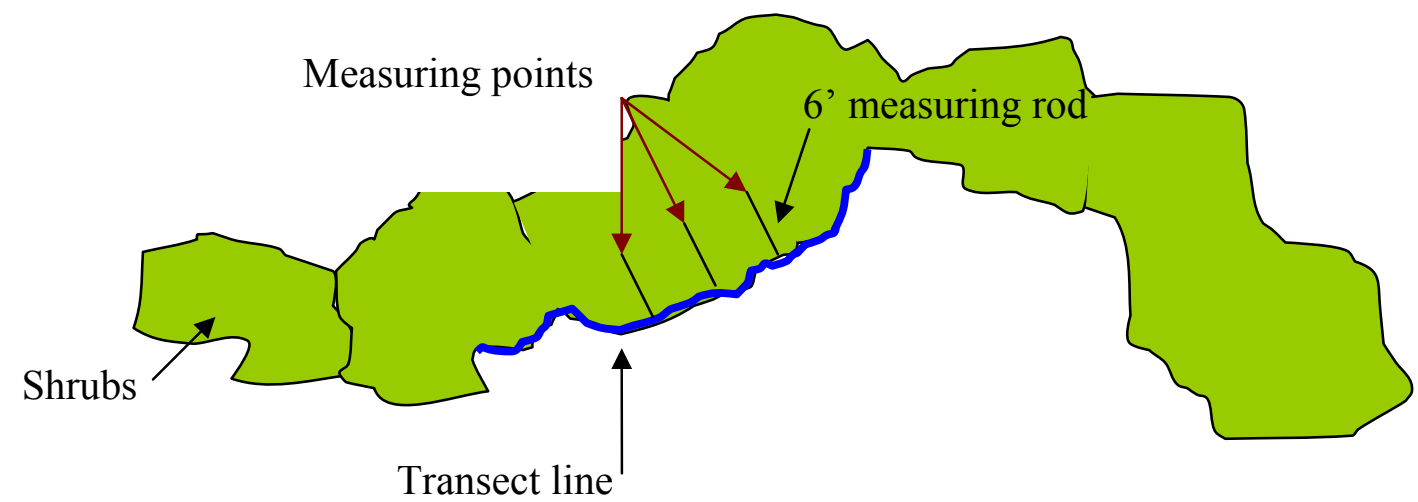

Figure 6. Modified point intercept layout example. 
4D: Complex shrub intercept is used to determine percent shrub cover in multi strata shrub communities. This method is generally associated with point intercept methods whereas overlapping shrubs are identified for each stratum. Percent cover is determined for each of four possible strata as well as total percent shrub cover and overlapping percent cover.

The complex shrub intercept method is identified by adding the suffix "4D" after the appropriate line or point intercept method. For example, "4B-1-4D designates that complex shrub point intercept measurements were taken at two foot intervals. Similarly, 4C-2-4D designates that modified point intercept at five foot intervals was used to determine percent shrub cover for strata in a complex shrub community.

\section{Shrub Height}

5. Shrubs are defined as woody vegetation including trees $<16$ feet in height unless otherwise defined in HEP models. The Regional HEP Team assumes that trees $<16$ feet tall function ecologically more like shrubs than trees.

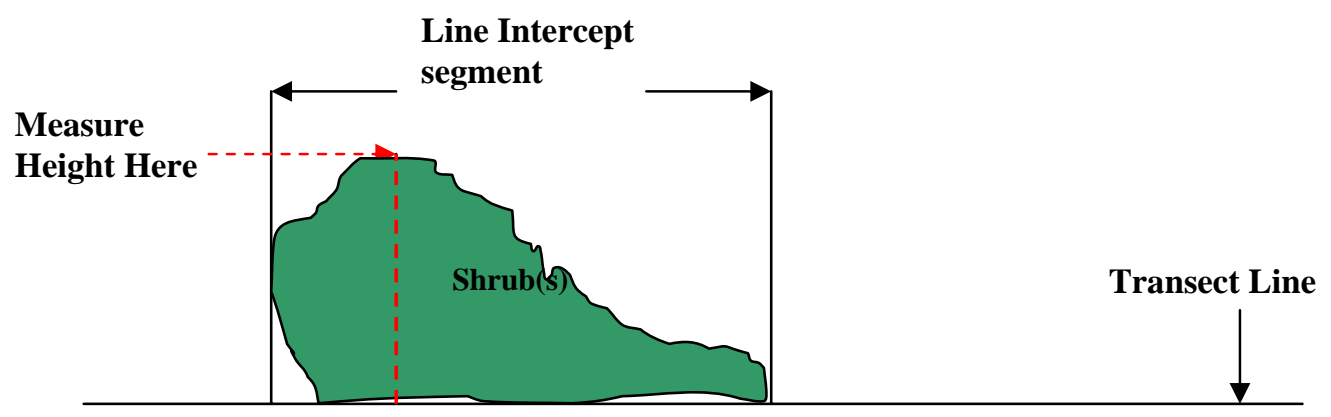

Horizontal View

Figure 7. Line intercept shrub height measurement example.

Shrub height is measured in $10^{\text {ths }}$ of feet at the highest point for each uninterrupted line intercept segment as depicted in Figure 7, or the highest point that crosses each point intercept interval mark on the transect tape (Figure 8).

In structurally complex (overlapping) shrub communities, height is measured for each stratum (maximum of four) as illustrated in Figure 9. It is assumed that shrub height measurements correspond to the method used to determine percent shrub cover. For example, if percent shrub cover is determined using the line intercept 
method (Figure 4), then it is assumed that shrub height will be obtained as illustrated in Figure 7.
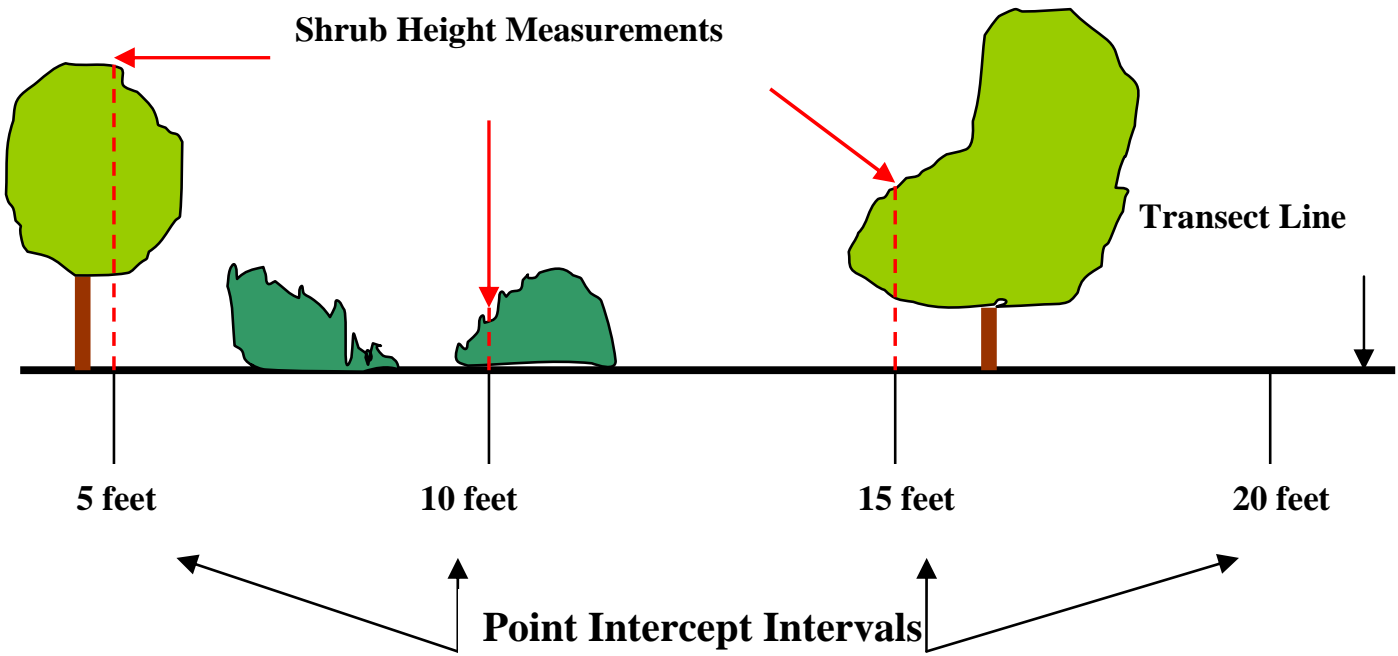

Figure 8. Point intercept shrub height example.

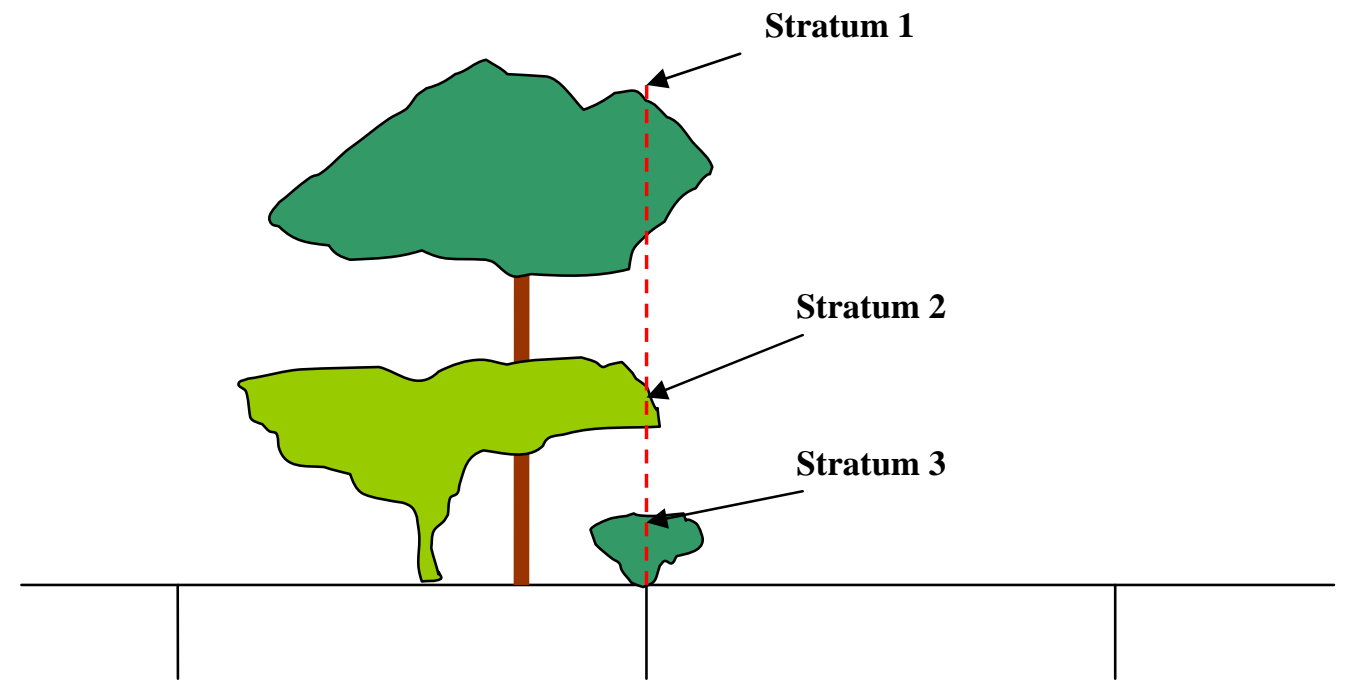

Figure 9. Complex shrub community shrub height measurement example. 


\section{Tree Measurements}

\section{Percent Canopy Cover}

6. Tree canopy cover measurements are recorded at five or ten foot intervals with a densitometer (point intercept). Measurement intervals are determined by visually estimating tree canopy closure prior to initiating the survey. If estimated canopy closure is $<20 \%$ and estimated transect length $\leq 900$ feet, measurements are recorded at five-foot intervals; if estimated canopy closure is $>20 \%$ and estimated transect length is $\geq 600$ feet, ten-foot intervals are used. The size of the sample area strongly influences transect length. In small areas, data from several short (300 foot) transects may be "pooled" in order to determine percent tree canopy cover. As with shrubs, sampled trees are identified by species and the sampling unit is a 100 foot segment of the transect.

6A: 5 ' interval

6B: 10 ' interval

\section{Height}

7. Tree height is determined generally using a clinometer. In open areas, an electronic height measurement instrument may be used. Measurements are taken at the beginning and end of each transect and at 100 foot intervals. Additional samples may be taken if needed. HEP model variable requirements determine the extent of tree height measurements e.g., multi-canopy, overstory, etc.

Basal Area

8. Tree basal area data is collected at 100 -foot intervals using a "factor 10" prism. Each 100-foot interval basal area observation (all tree "hits" at each 100-foot point) is considered an independent sample.

\section{Snag DBH}

9. Snag data is collected on belt transects. RHT members collect snag data in conjunction with tree canopy closure measurements using the same baseline transect. The diameter breast height (DBH) of all snags present within tenth-acre belt transects paralleling the baseline transect is measured. Either the actual DBH is recorded, or snag data is reported by class e.g., 5 snags $<4$ " DBH, 2 snags $>20$ " DBH etc.

Belt transects are 44 feet wide by 100 feet long i.e., 22 feet on each side of the baseline transect. Belt transect layout is depicted in Figure 10. As with shrubs and trees, the sampling unit is each 100-foot segment. 


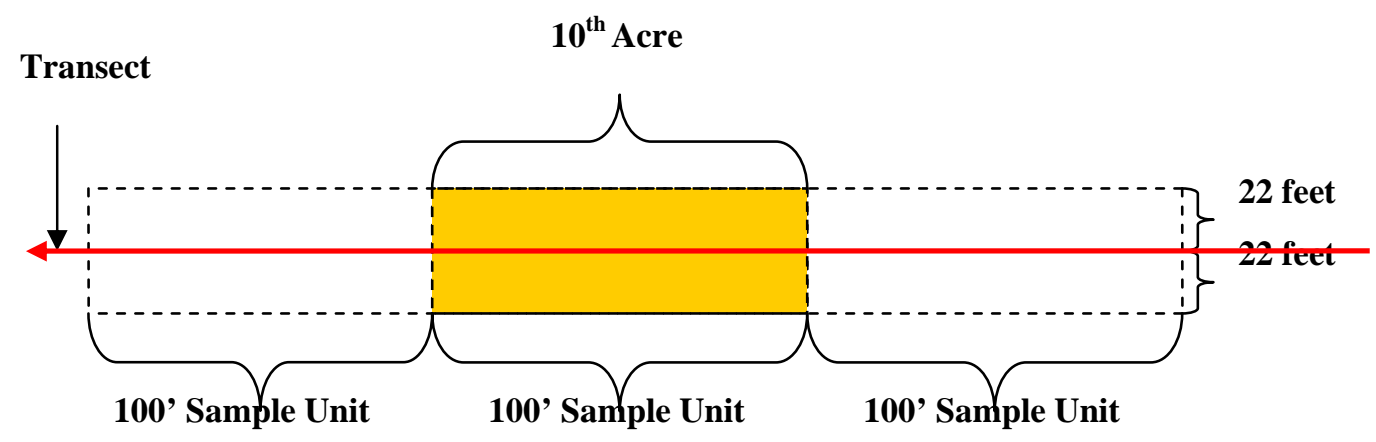

Figure 10. Belt transect layout diagram.

\section{Sample Size Determination}

The process for determining sample size (transect length) varies based on the variable measured. Shrub and tree cover and grid sample sizes are estimated as follows:

The amount of cover within each 100 foot sample unit is divided by sample unit length to obtain percent shrub/tree cover per sample unit (e.g. 10 feet of cover/100 feet $=10 \%$ shrub cover). The standard deviation for each transect is calculated for percent cover data from transect sample units. Sample size (transect length) is then determined through use of the following equation (Avery 1994):

$$
\mathrm{n}=\frac{\mathrm{t}^{2} \mathrm{~s}^{2}}{\mathrm{E}^{2}}
$$

Where: $\mathrm{t}=\mathrm{t}$ value at the 95 percent $(0.05)$ confidence interval for the appropriate degrees of freedom (df); $\mathrm{s}=$ standard deviation; and $\mathrm{E}=$ desired level of precision, or bounds ( \pm 10 percent). Confidence intervals may vary from 80 percent $(0.20)$ to 95 percent $(0.05)$ depending on habitat variable heterogeneity and project management needs. The same method is used to determine sample size for micro plot samples based on total percent cover for herbaceous species. 


\section{References}

Avery, T.E., H. E. Burkhart. 1994. Forest measurements. $4^{\text {th }}$ edition. John Wiley and Sons. New York, NY.

BLM. 1998. Measuring and monitoring plant populations. BLM Technical Reference 1730-1. BLM National Business Center. Denver, CO. 477 p.

Block, W.M., W.L. Kendall, M.L. Morrison, and M. Dale Strickland. 2001. Wildlife study design. Springer Press. New York, NY. 210 p.

Hays, R. L., C. Summers, and W. Seitz. 1981. Estimating habitat variables. Western Energy and land Use Team. Fort Collins, CO: U.S. Fish and Wildlife Service.

Robel, R.J., J. N. Dayton, A.D. Hulbert. 1975. Relationship between visual obstruction measurements and weight of grassland vegetation. Journal of Range Management. 23: 295. 
West Foster Creek Expansion Project

\section{Appendix C - Transect Location Maps}

\section{North Bridgeport}

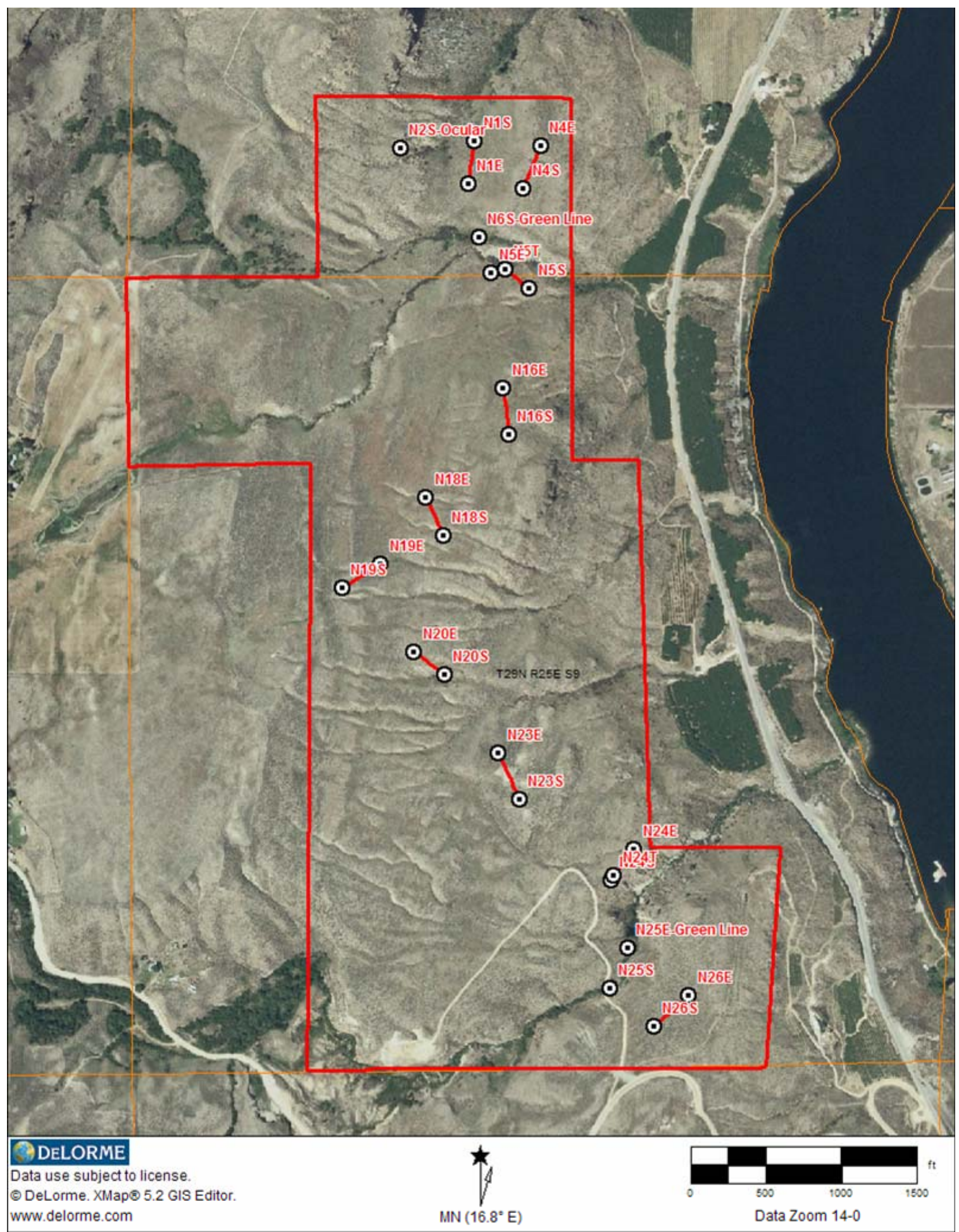


West Foster Creek Expansion Project

\section{Middle Bridgeport}

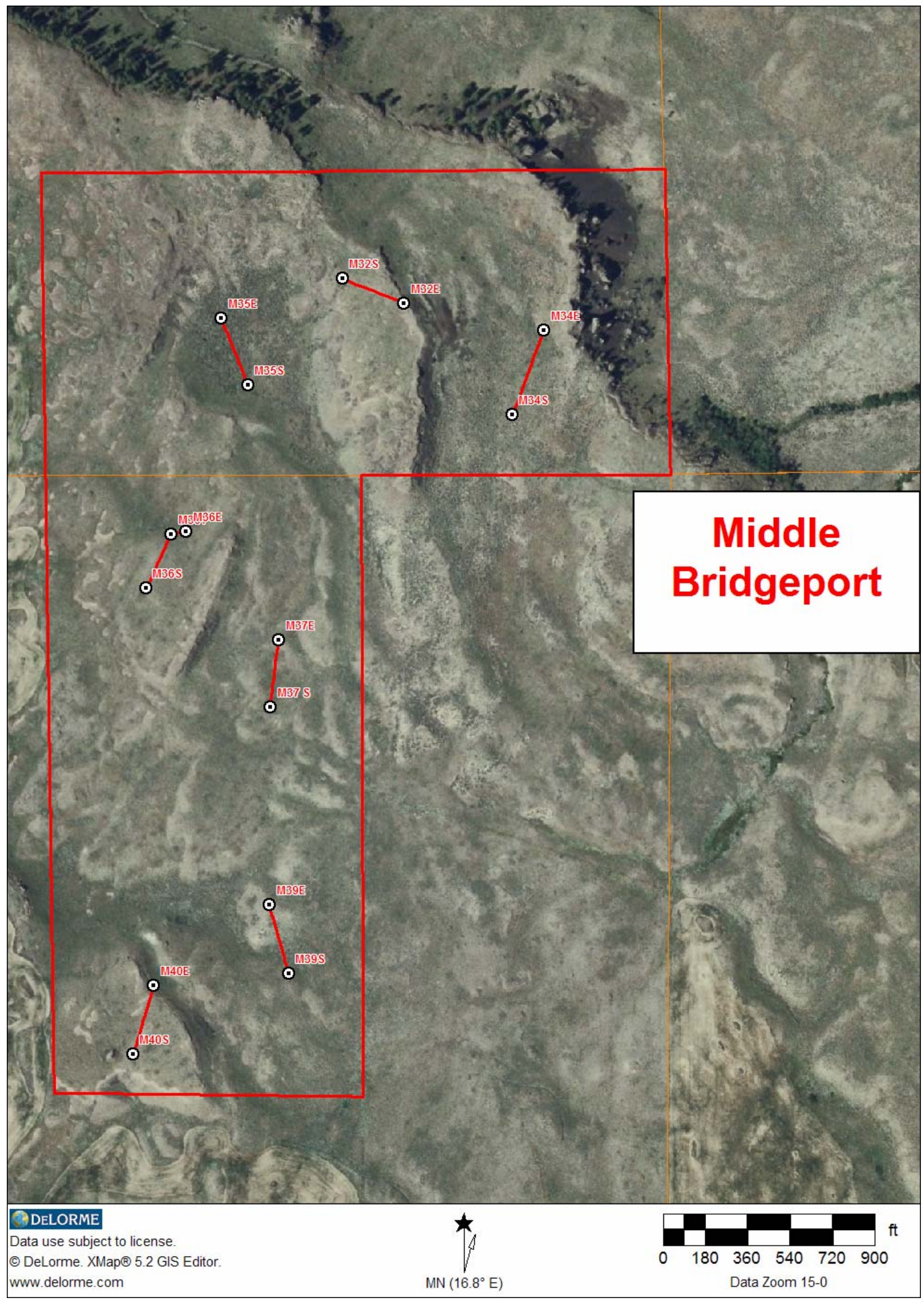


West Foster Creek Expansion Project

\section{McClain Lake}

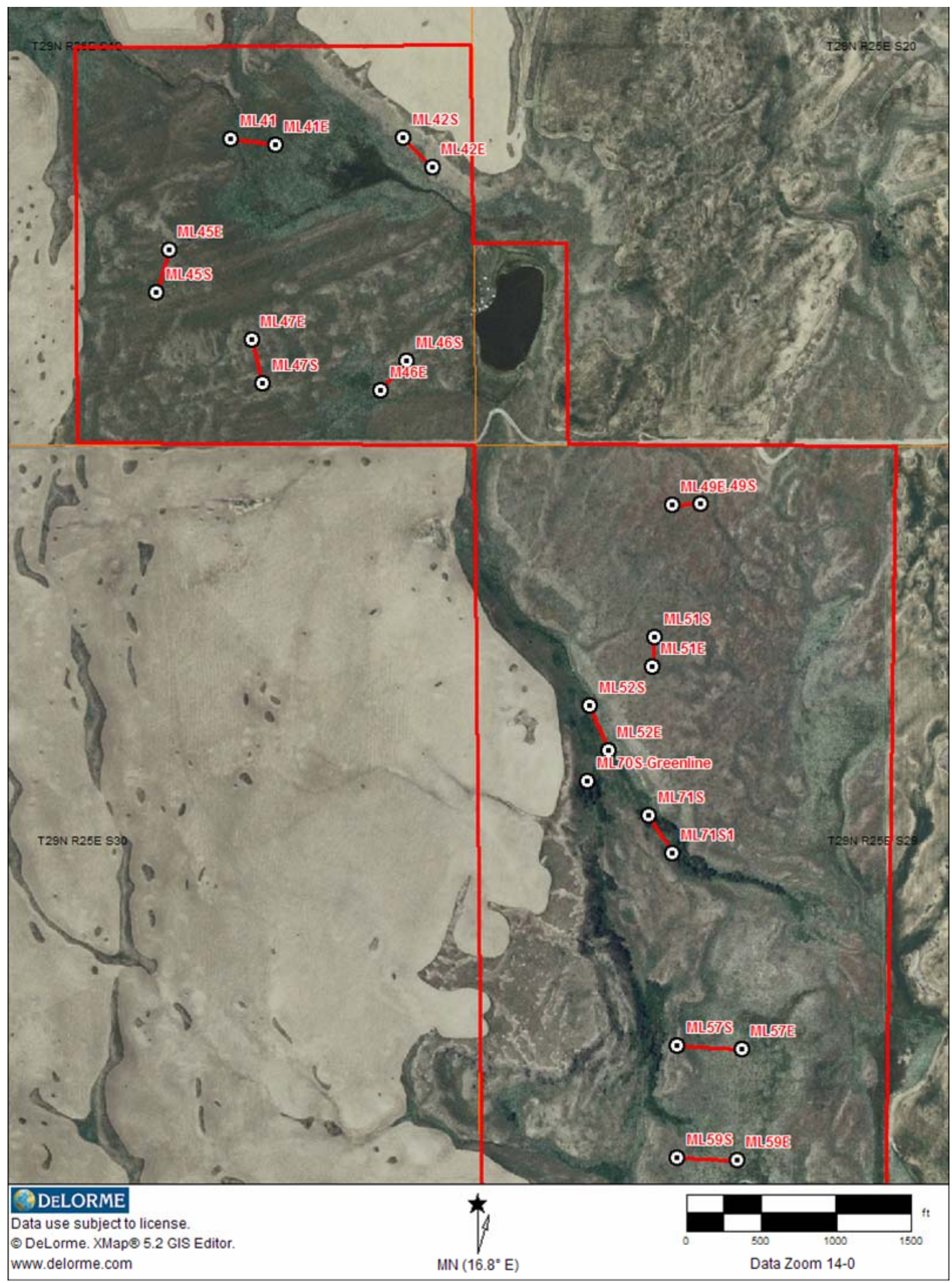


West Foster Creek Expansion Project

\section{Dezellum Lake}

Boundary data provided by Dan Peterson and John Talmadge - WDFW 2007

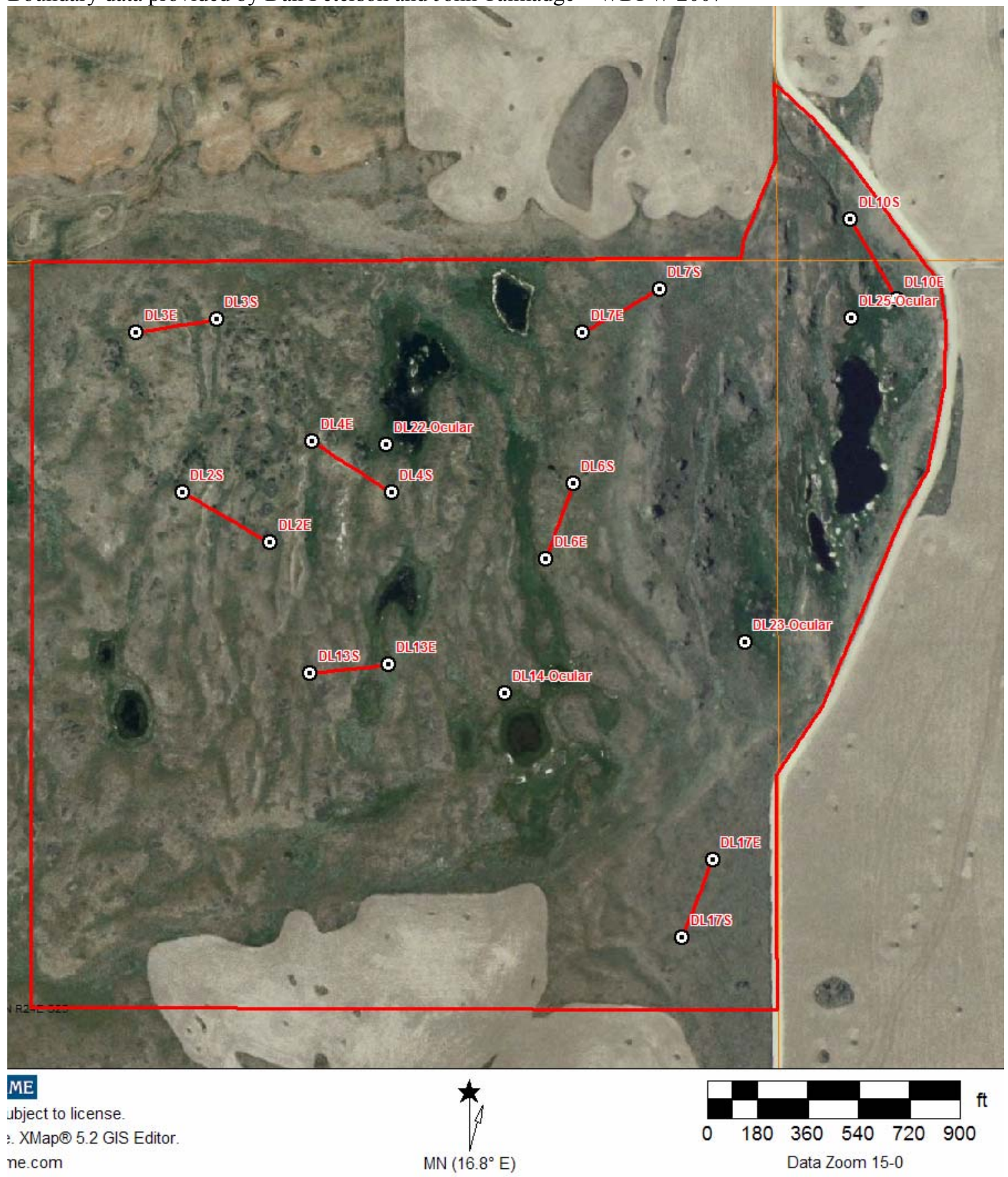


West Foster Creek Expansion Project

\section{JoJaCo}

Boundary data provided by John Talmadge-WDFW 2007

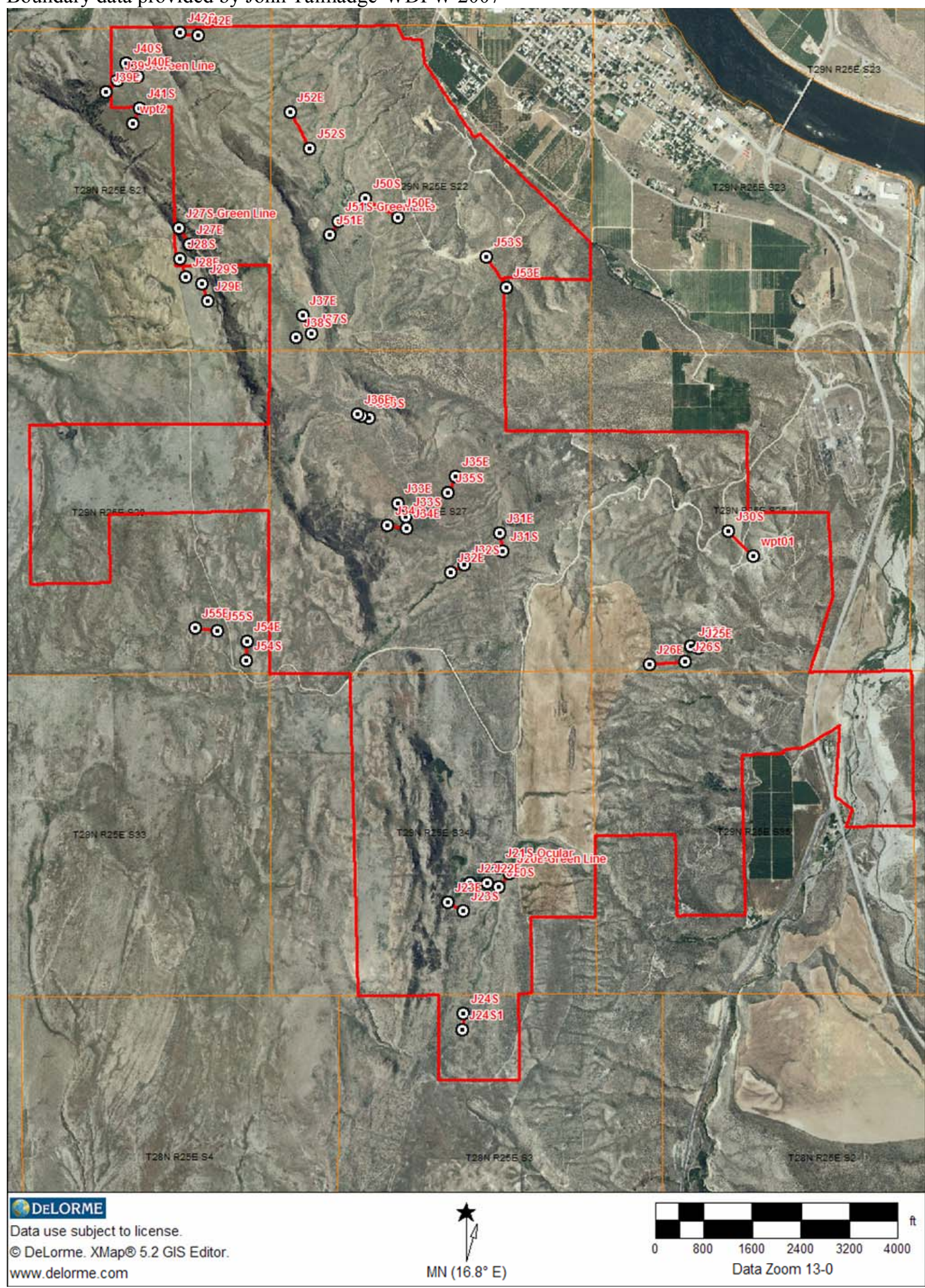


West Foster Creek Expansion Project

\section{JoJaCo - Large Scale Transect Maps}

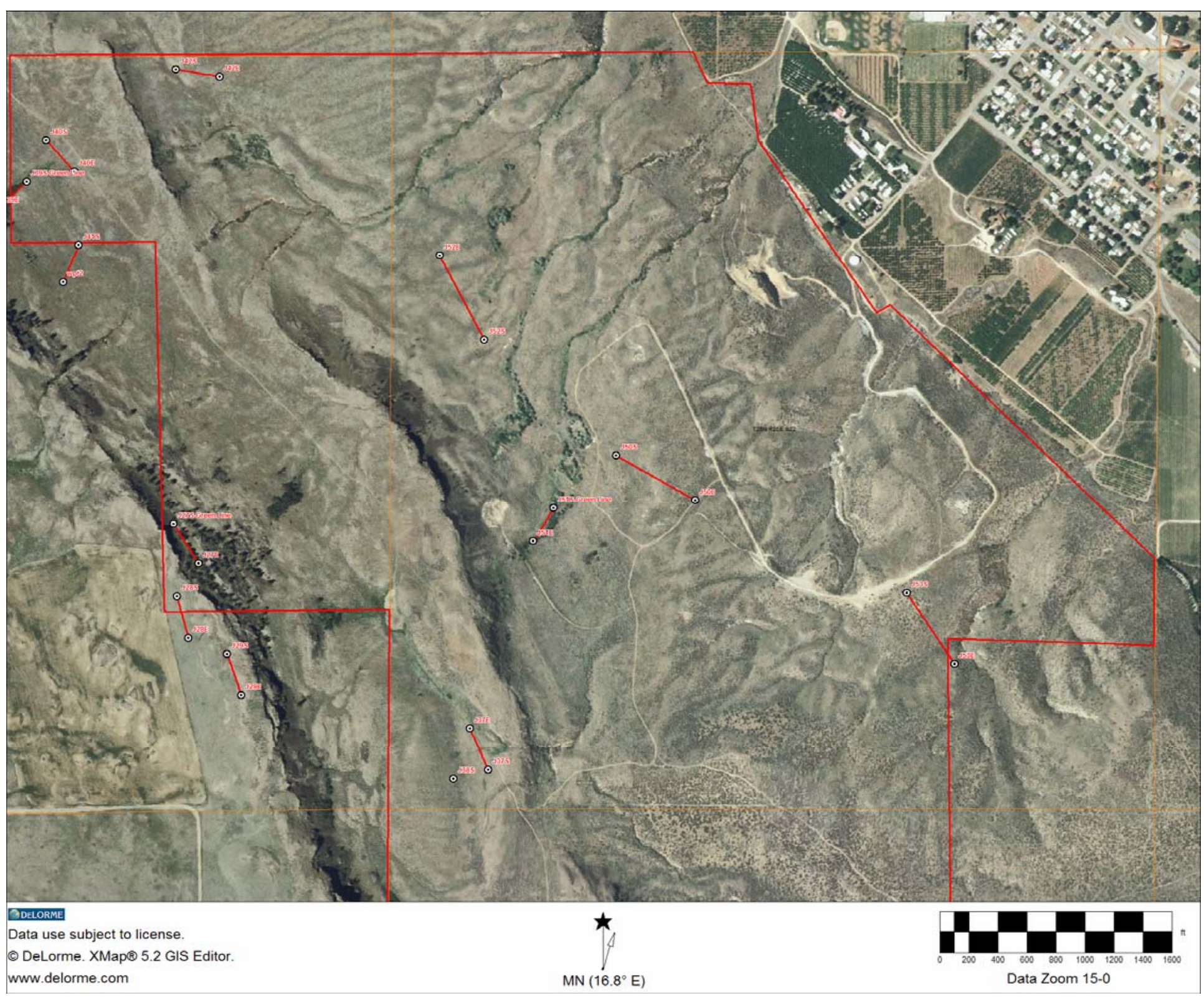


West Foster Creek Expansion Project

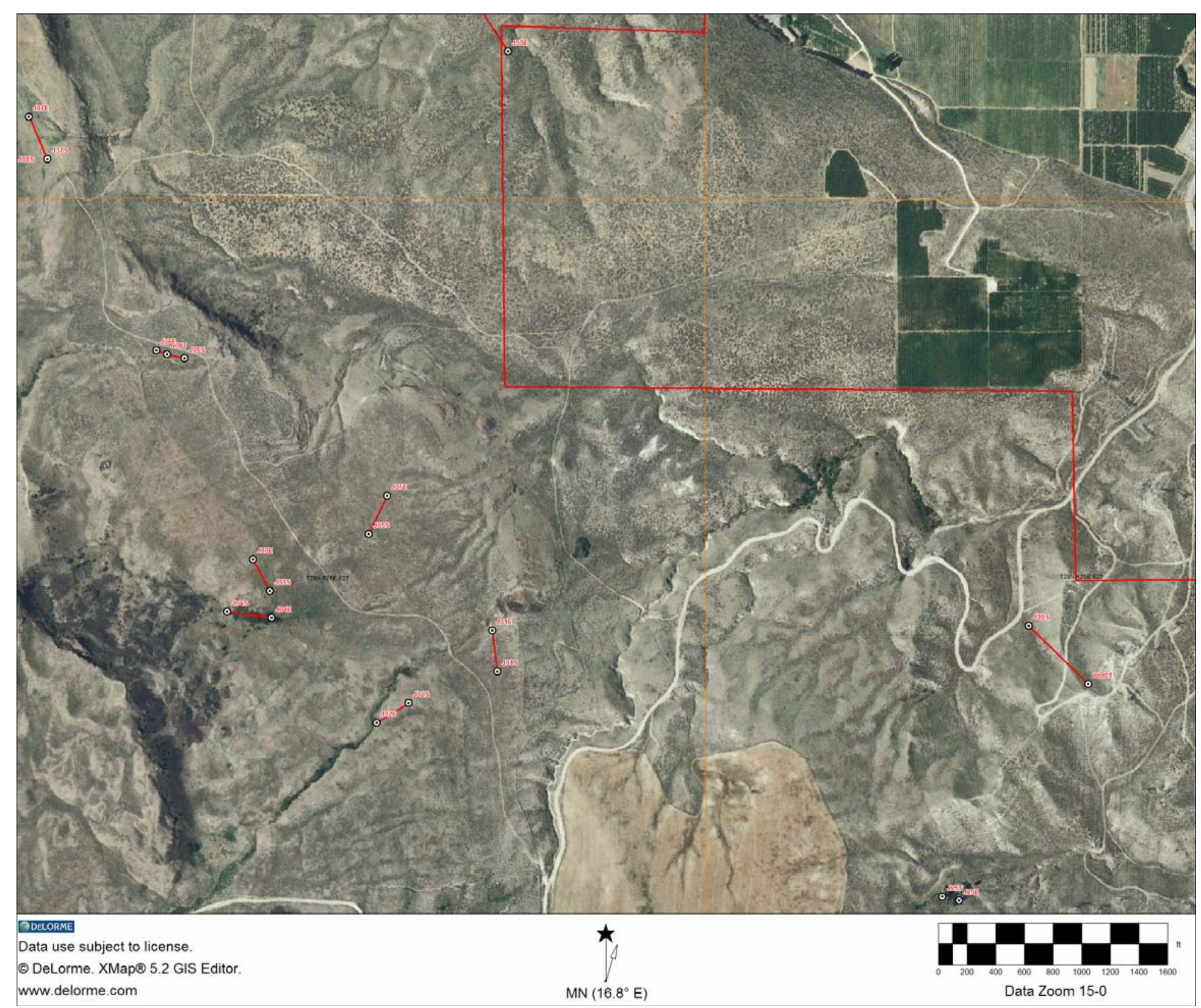


West Foster Creek Expansion Project

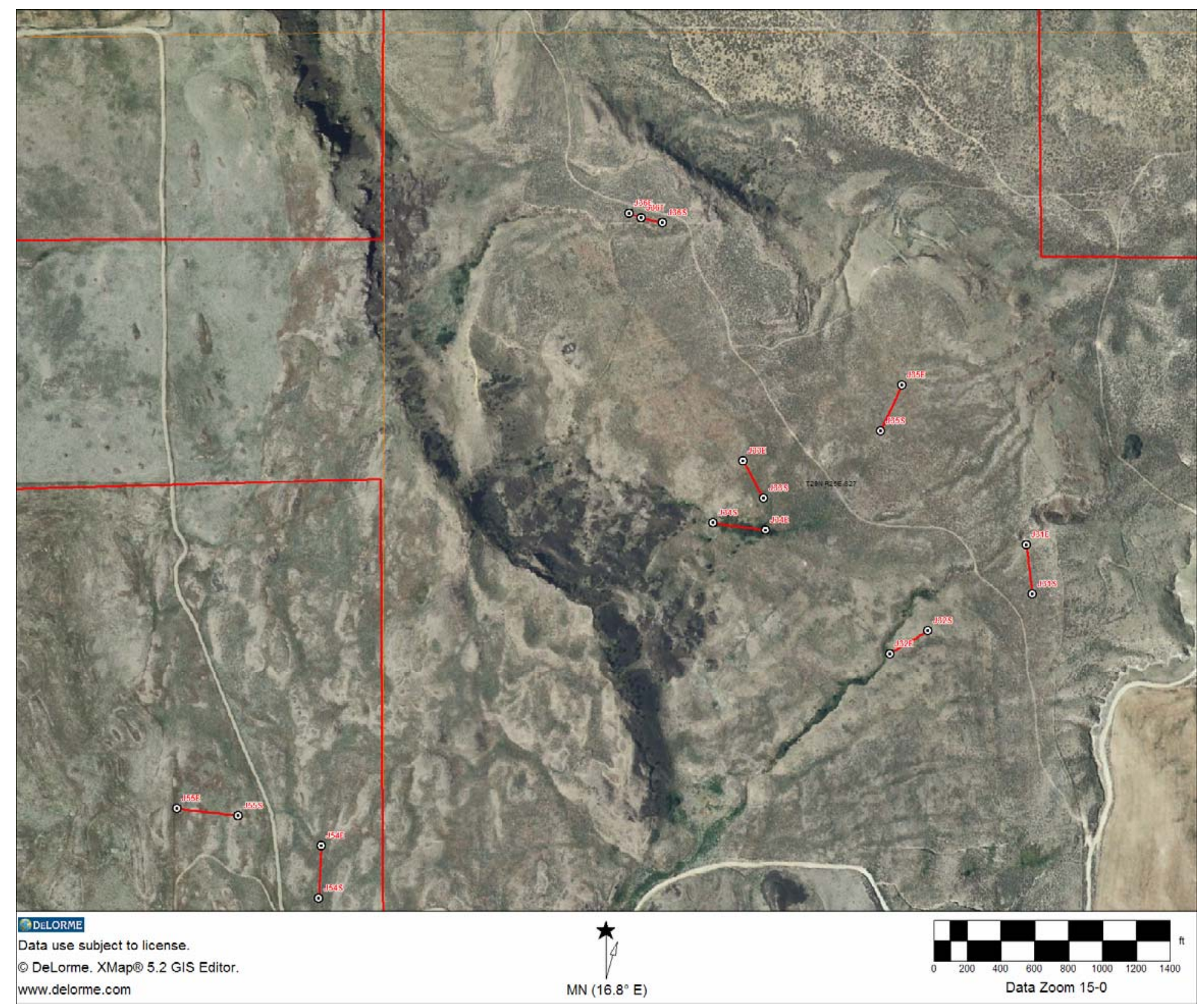


West Foster Creek Expansion Project

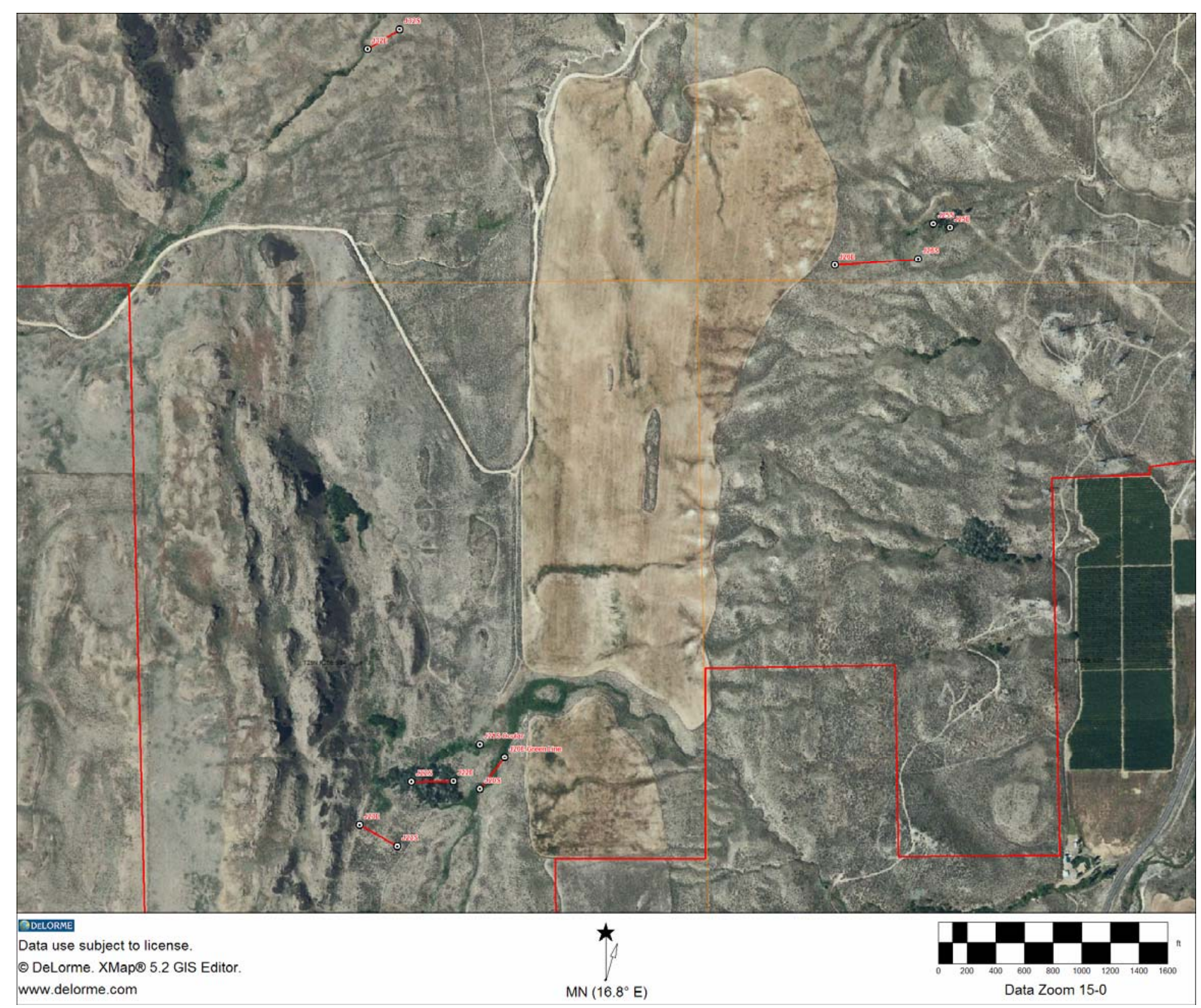


West Foster Creek Expansion Project

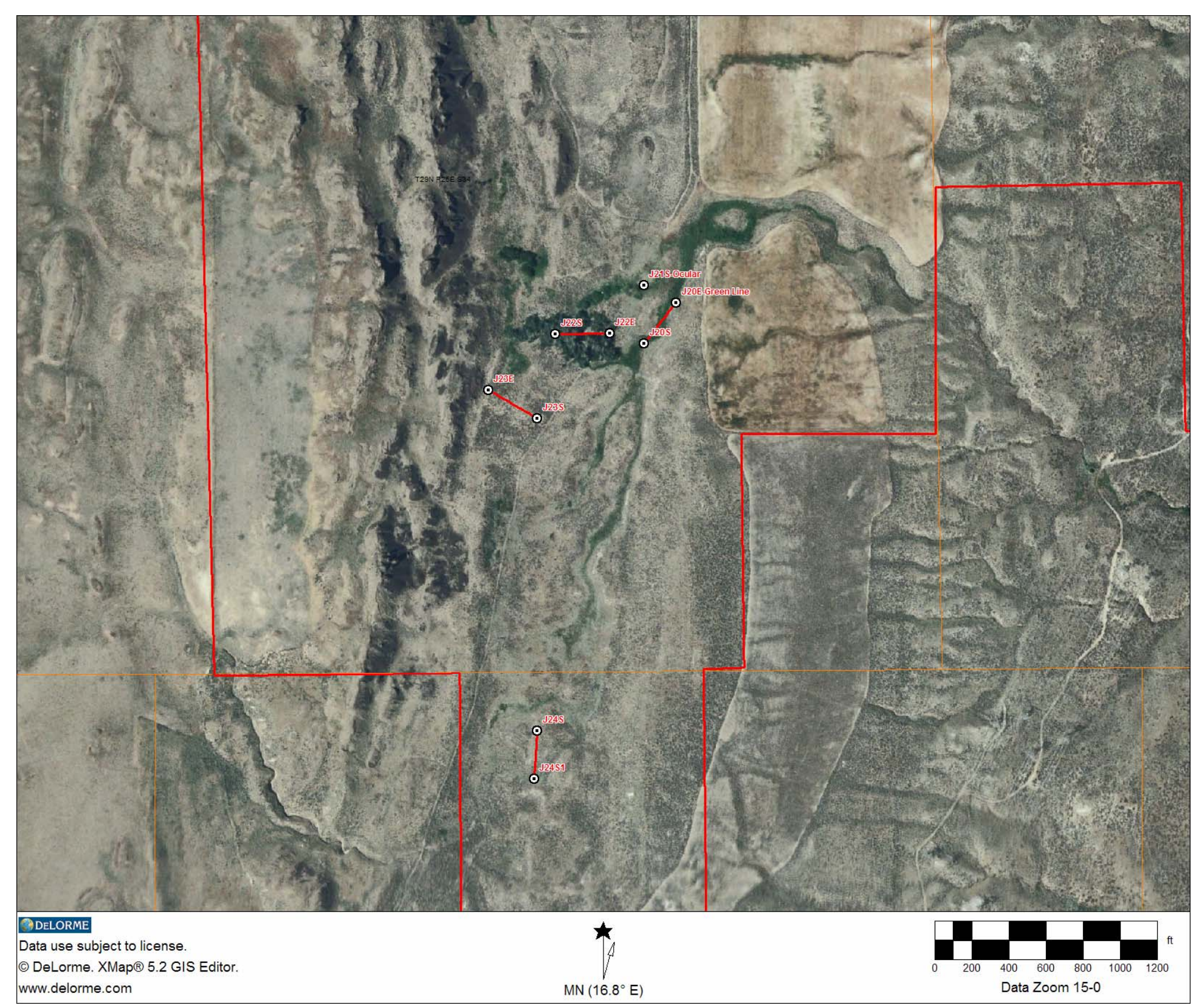


West Foster Creek Expansion Project

\section{Appendix D - Transect Photographs}

\section{North Bridgeport}

Transect 1

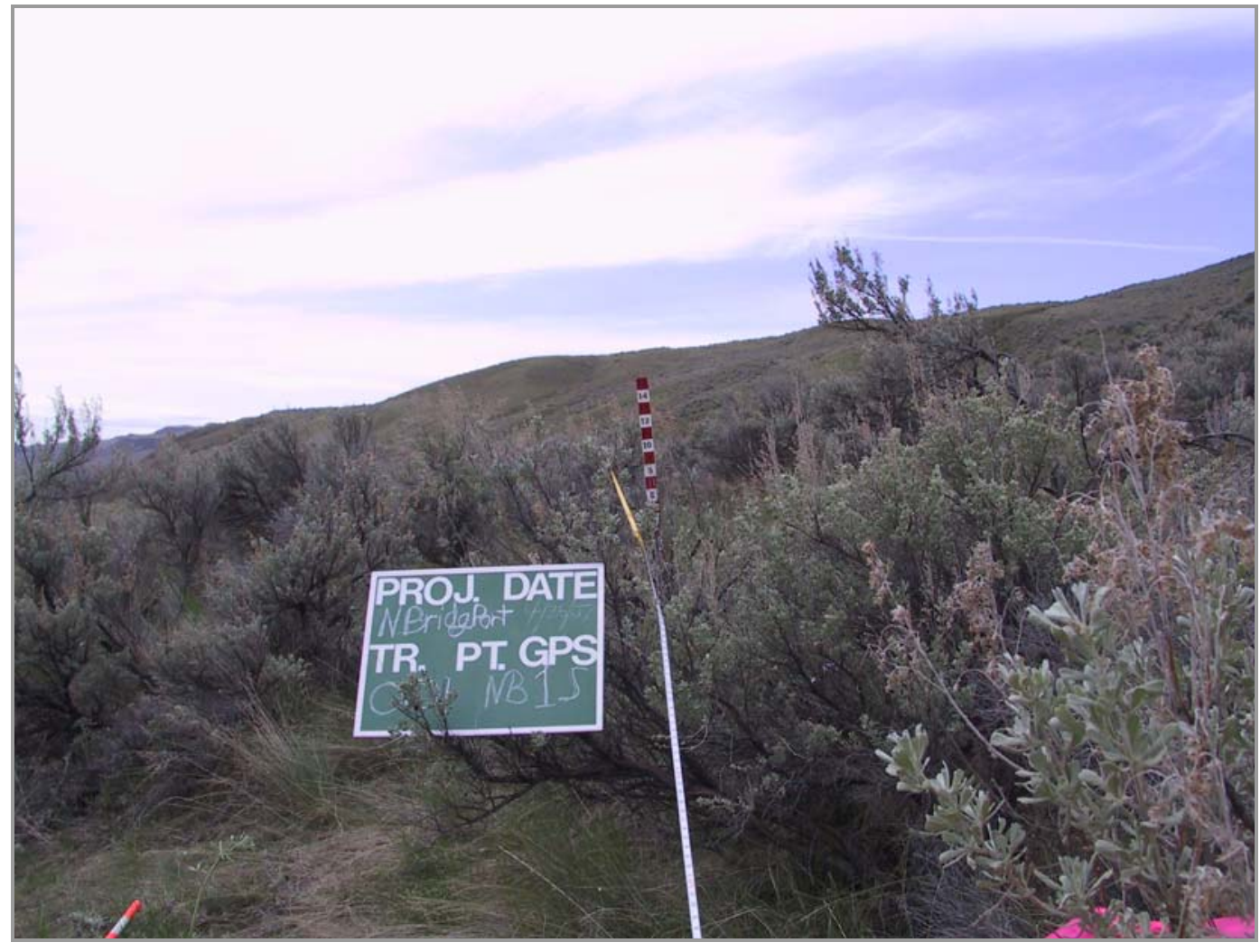

\section{Transect 2}

No photograph 
West Foster Creek Expansion Project

\section{Transect 4}

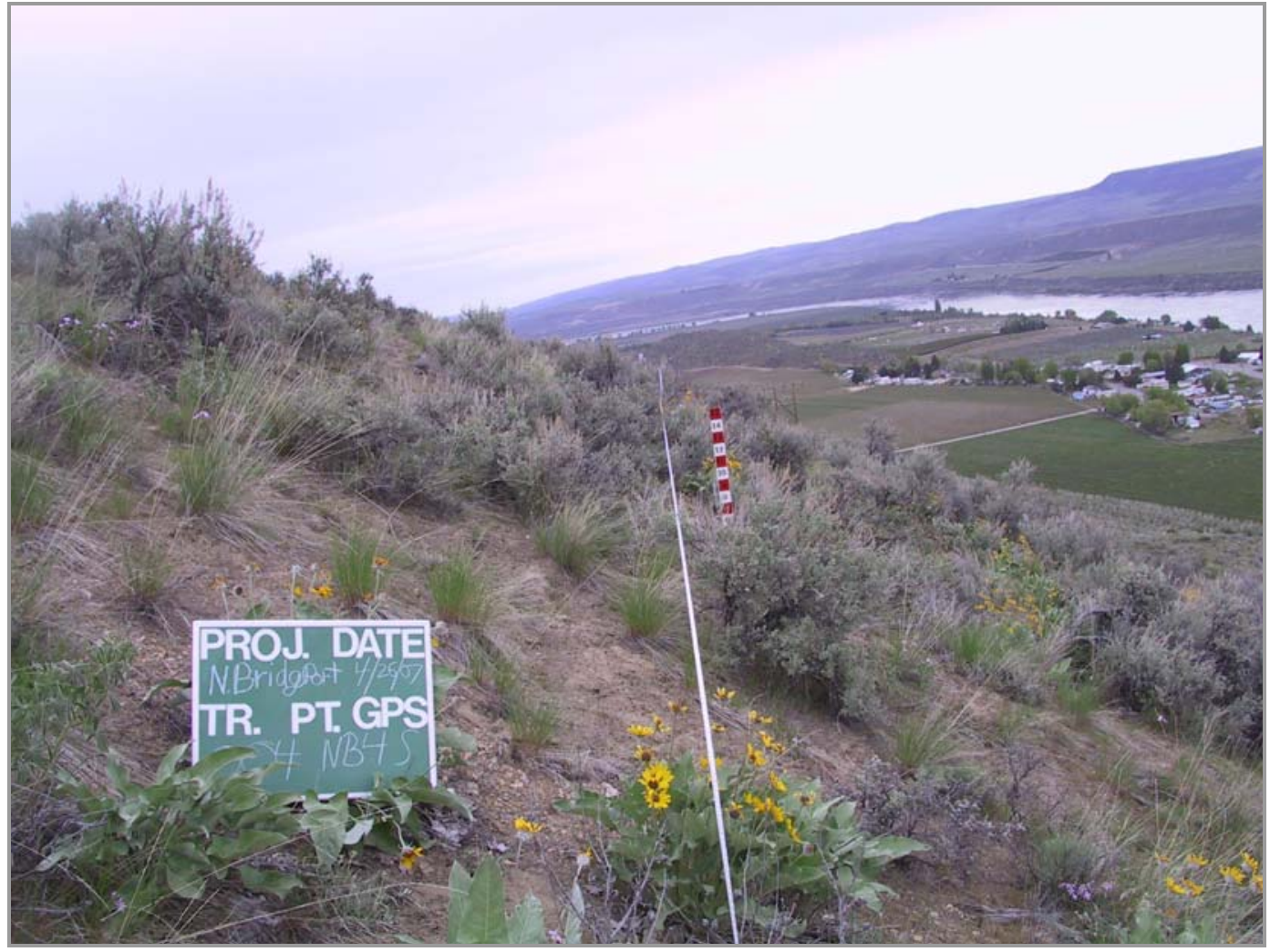

\section{Transect 5}

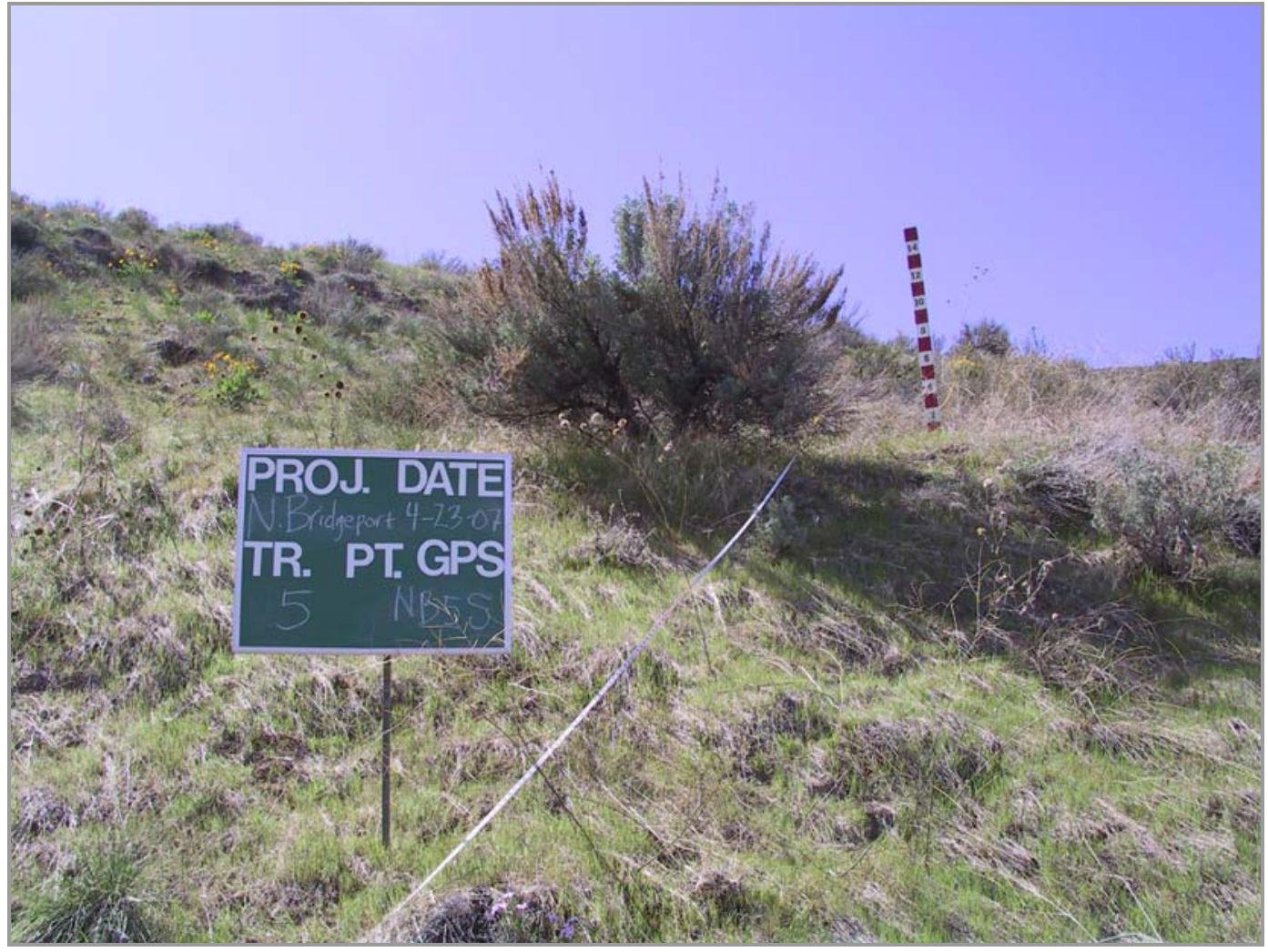


West Foster Creek Expansion Project

\section{Transect 6}

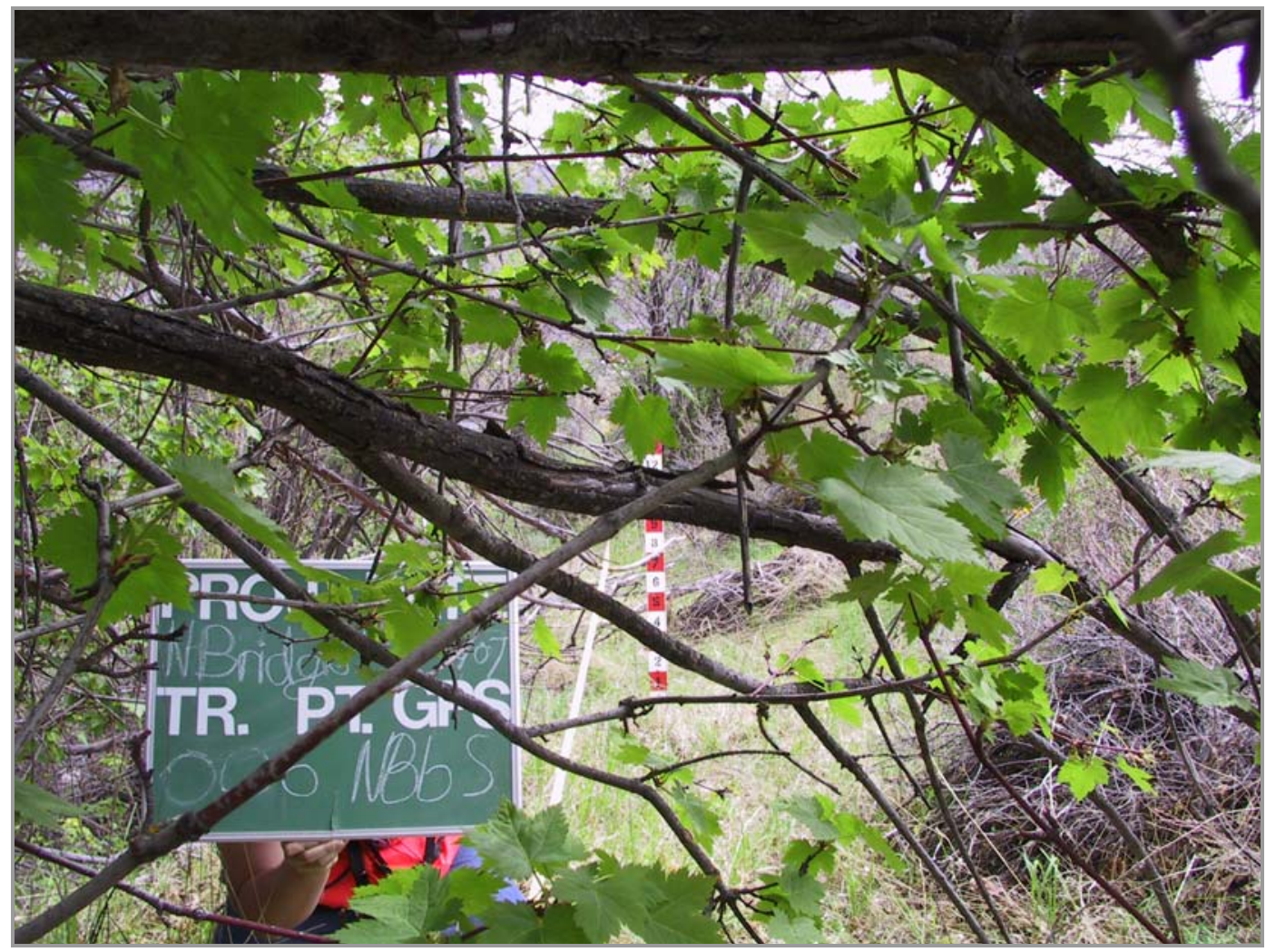

\section{Transect 16}

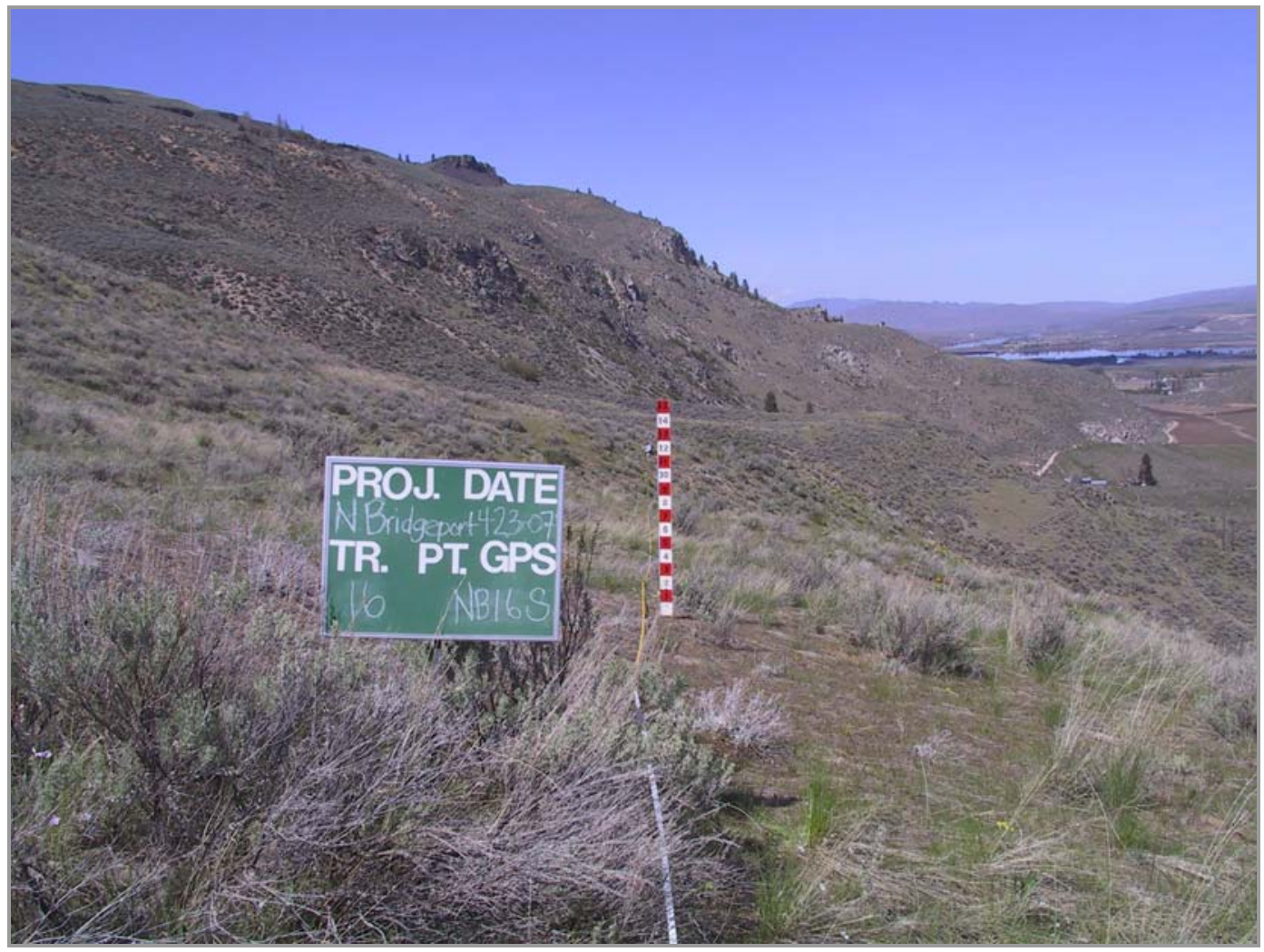


West Foster Creek Expansion Project

\section{Transect 18}

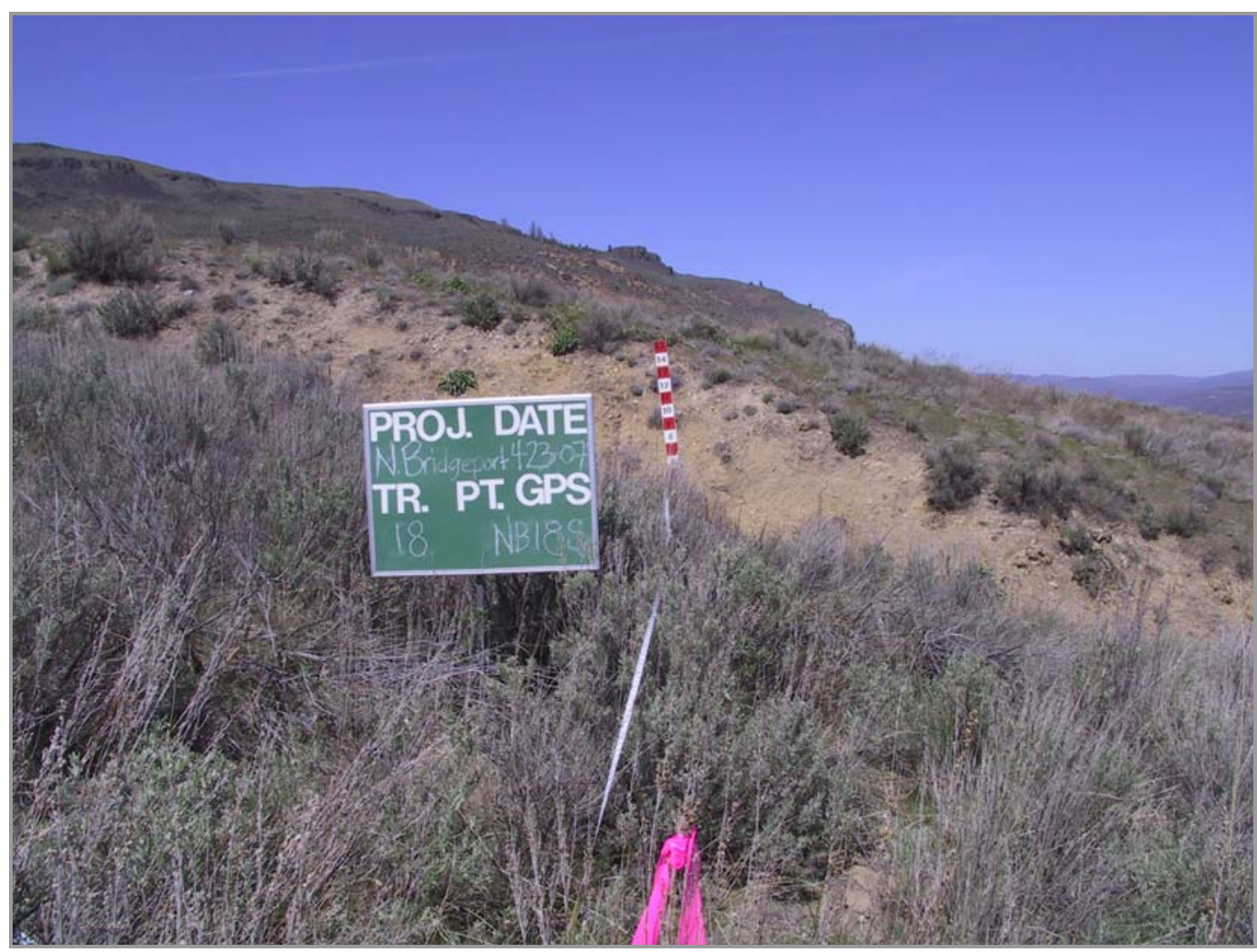

\section{Transect 19}

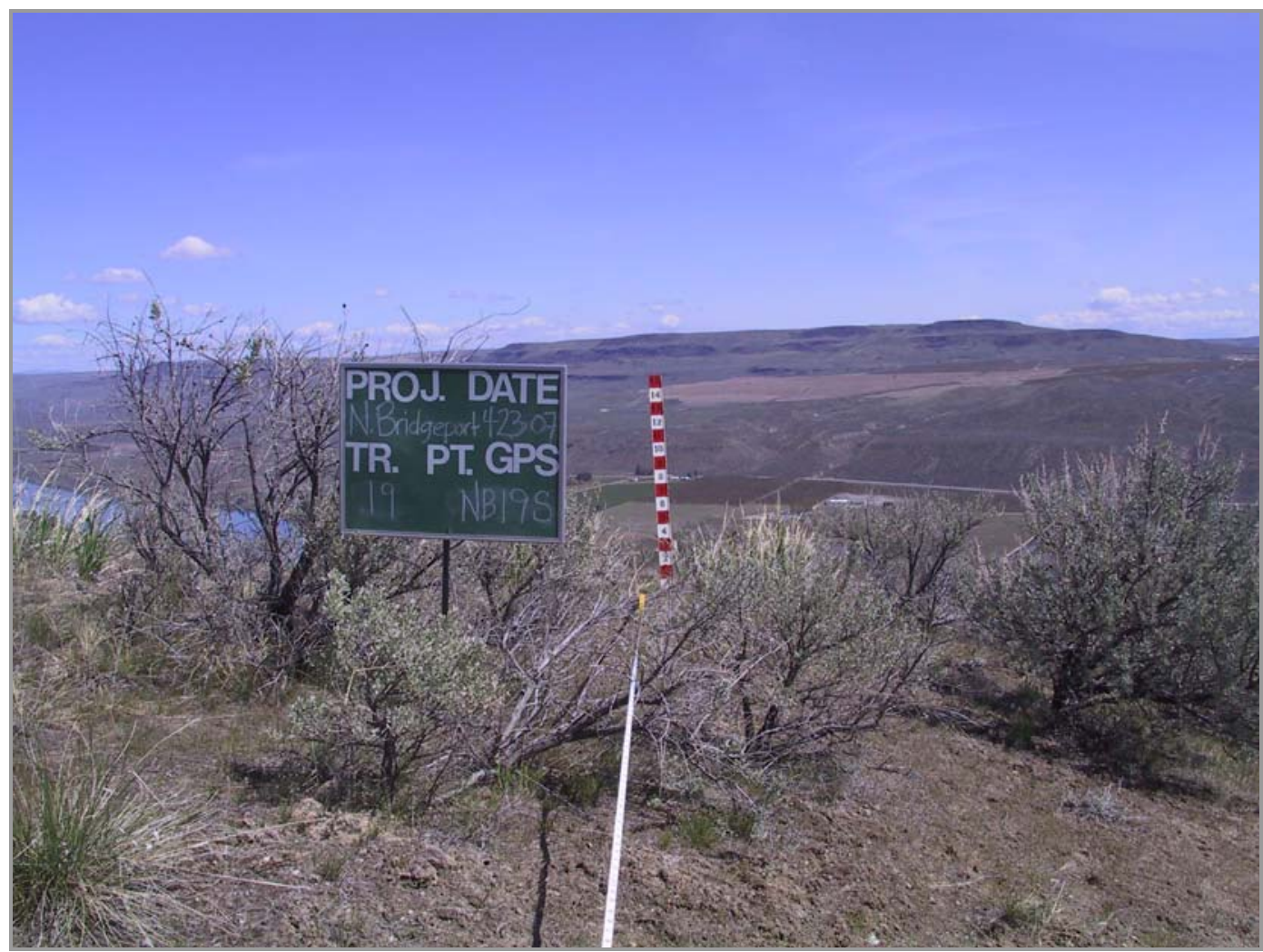


West Foster Creek Expansion Project

\section{Transect 20}

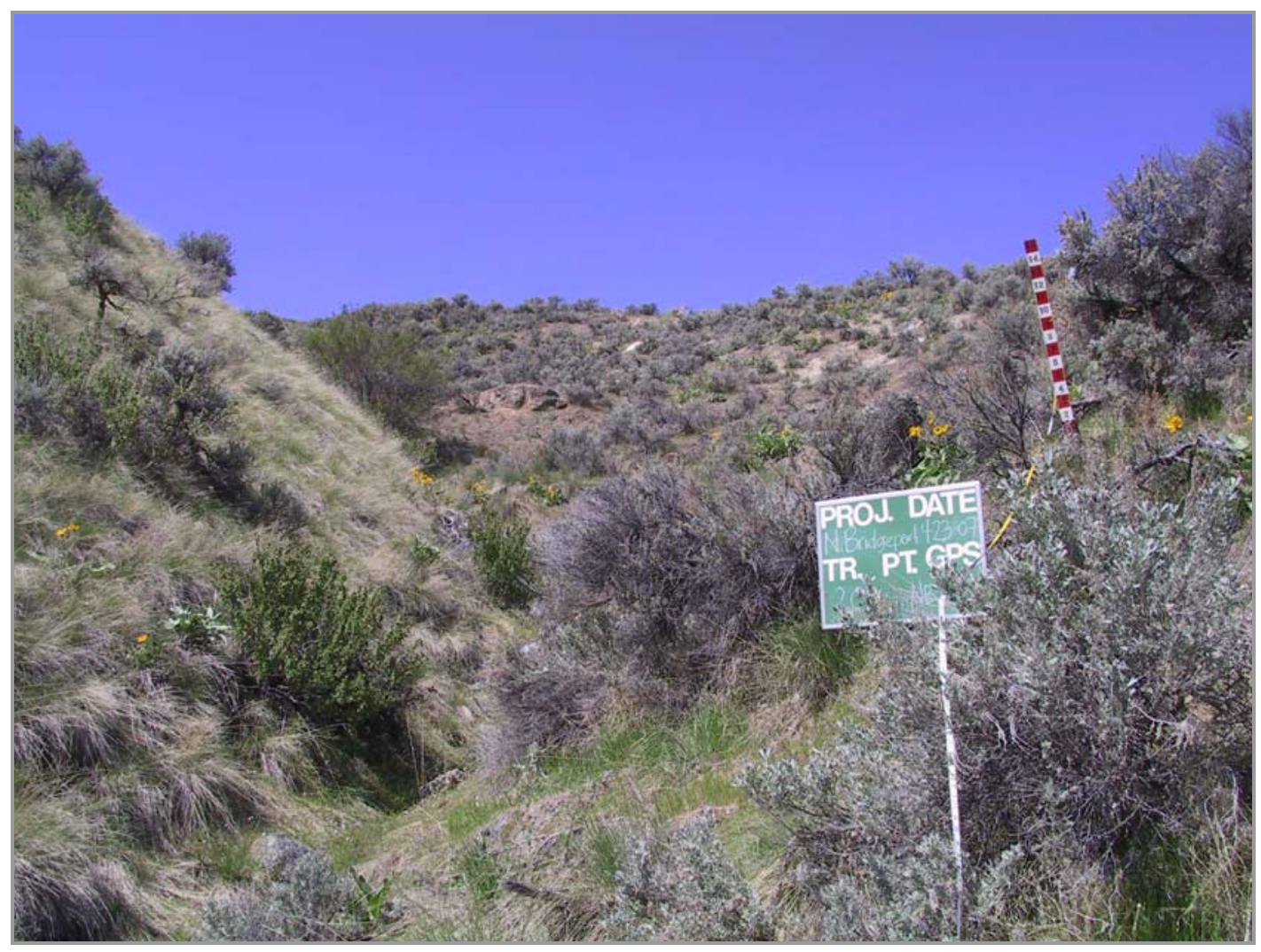

\section{Transect 23}

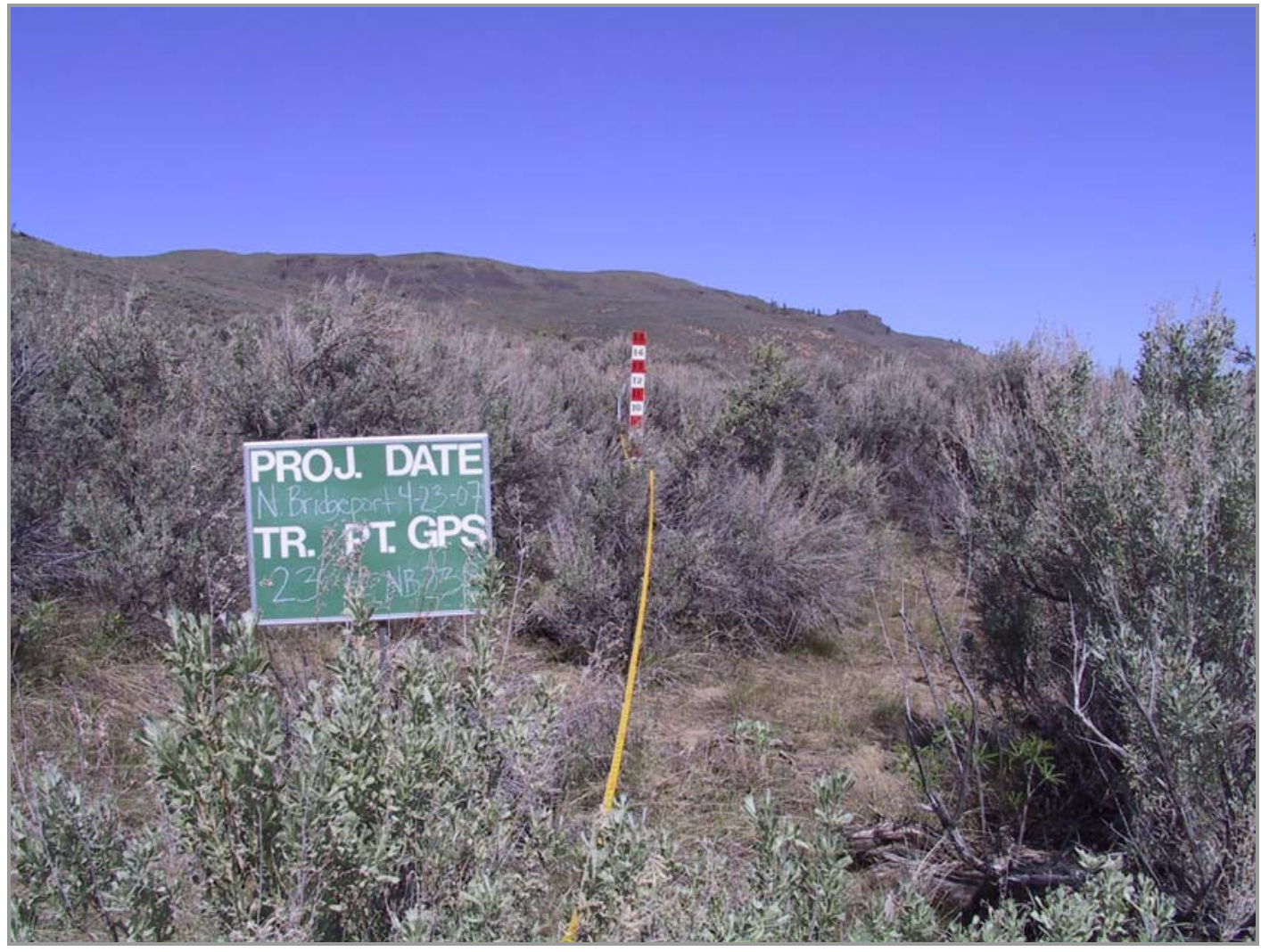


West Foster Creek Expansion Project

\section{Transect 24}

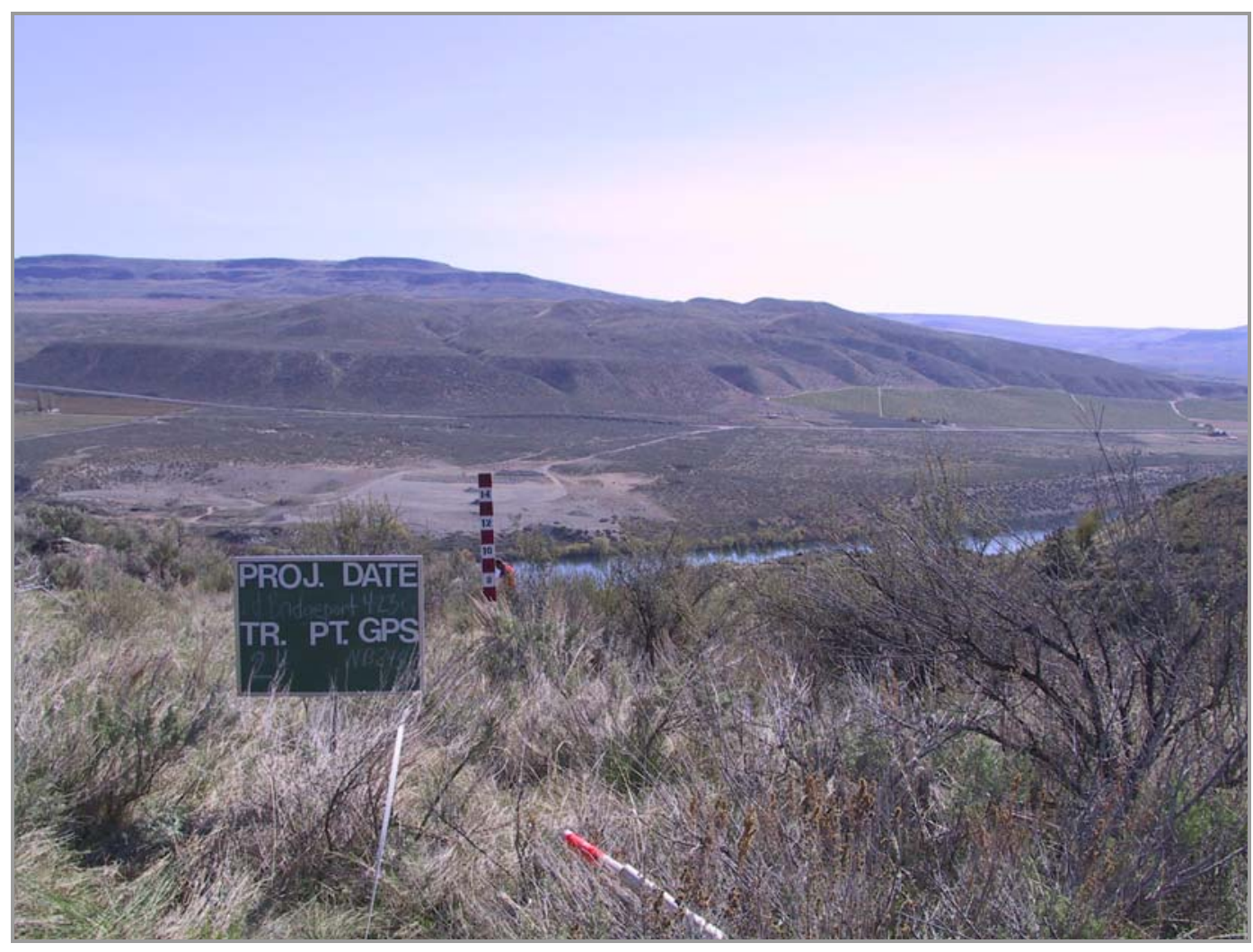

\section{Transect 25}

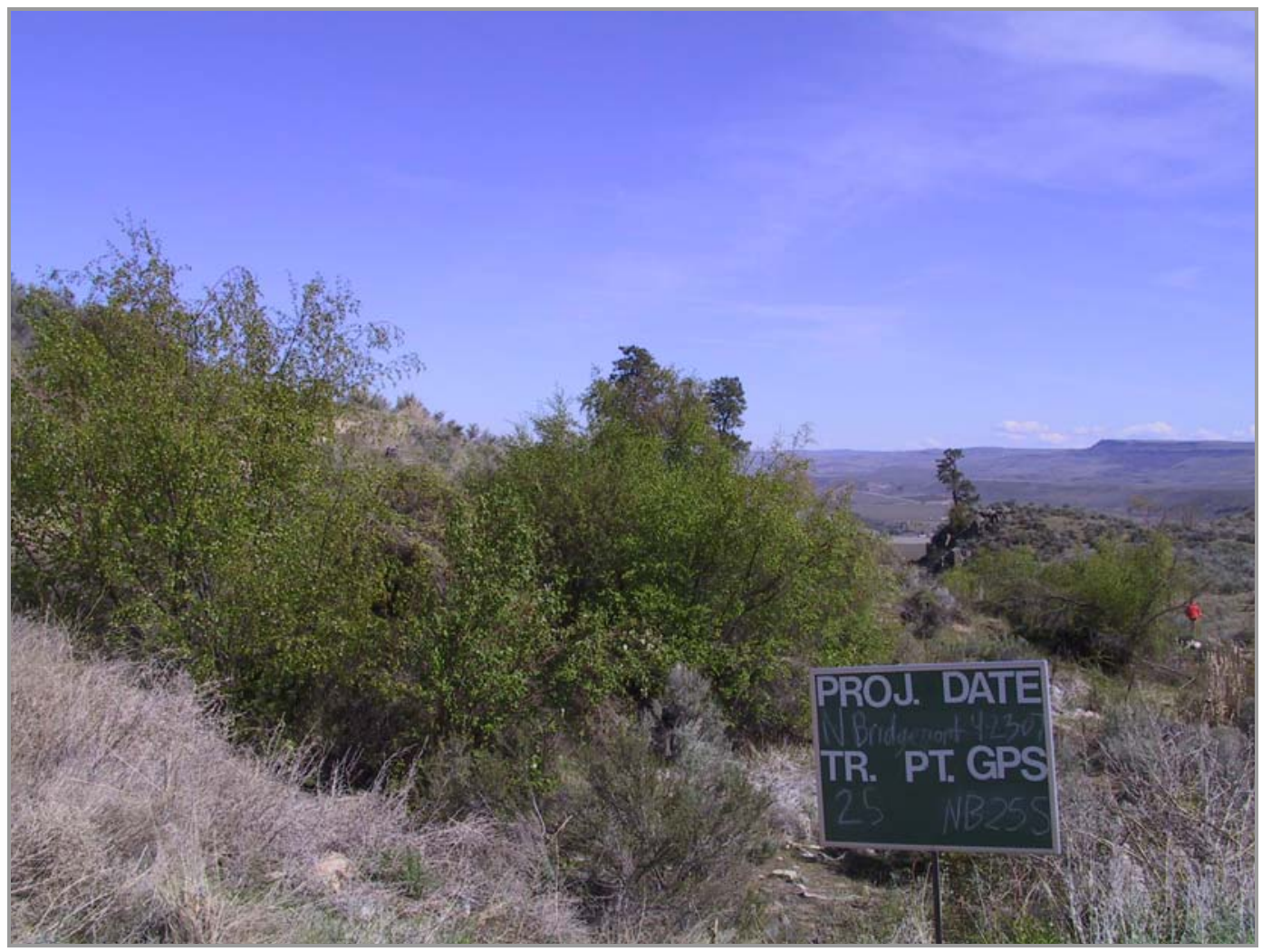


West Foster Creek Expansion Project

\section{Transect 26}

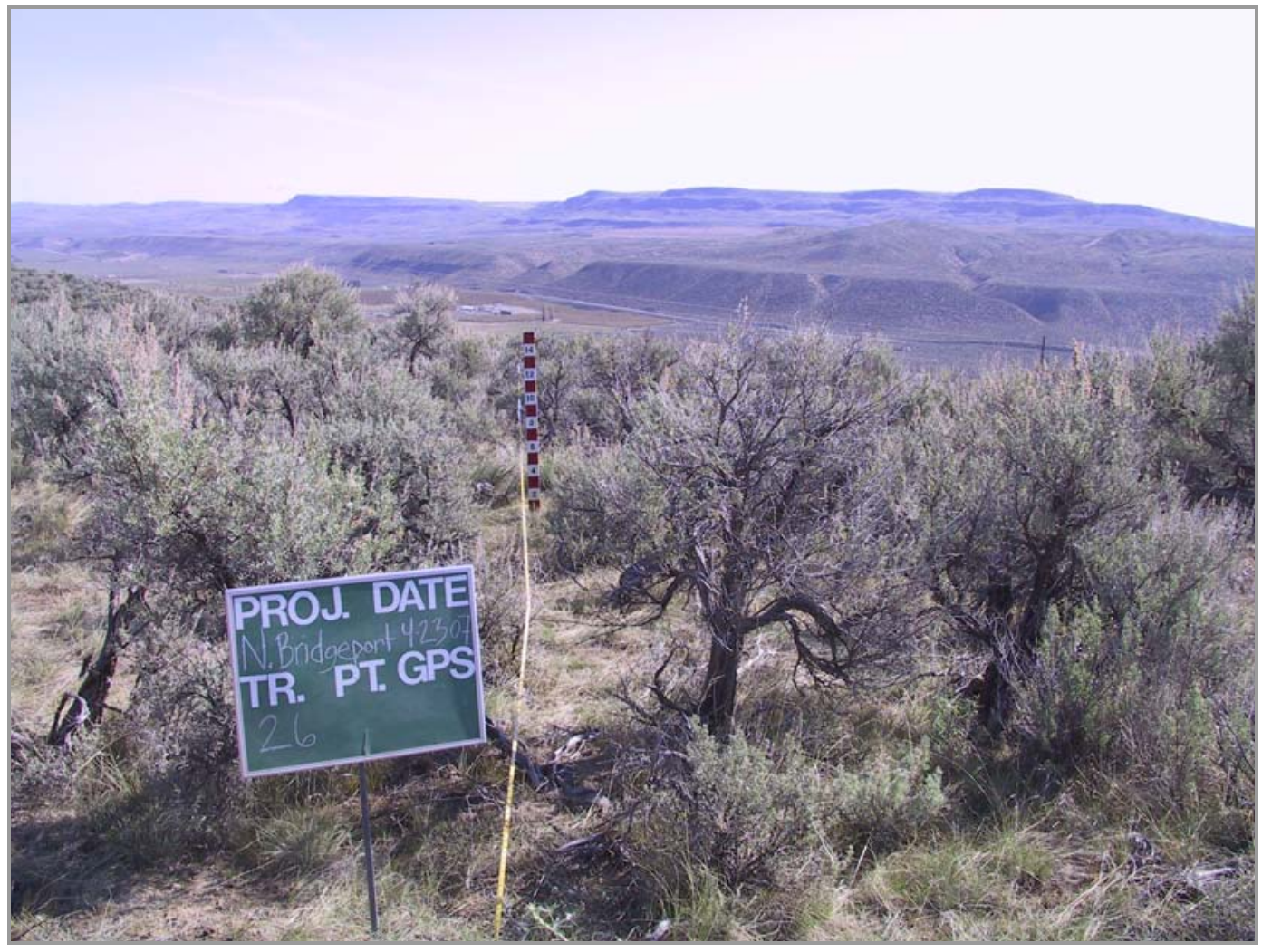


West Foster Creek Expansion Project

\section{Middle Bridgeport}

Transect 32

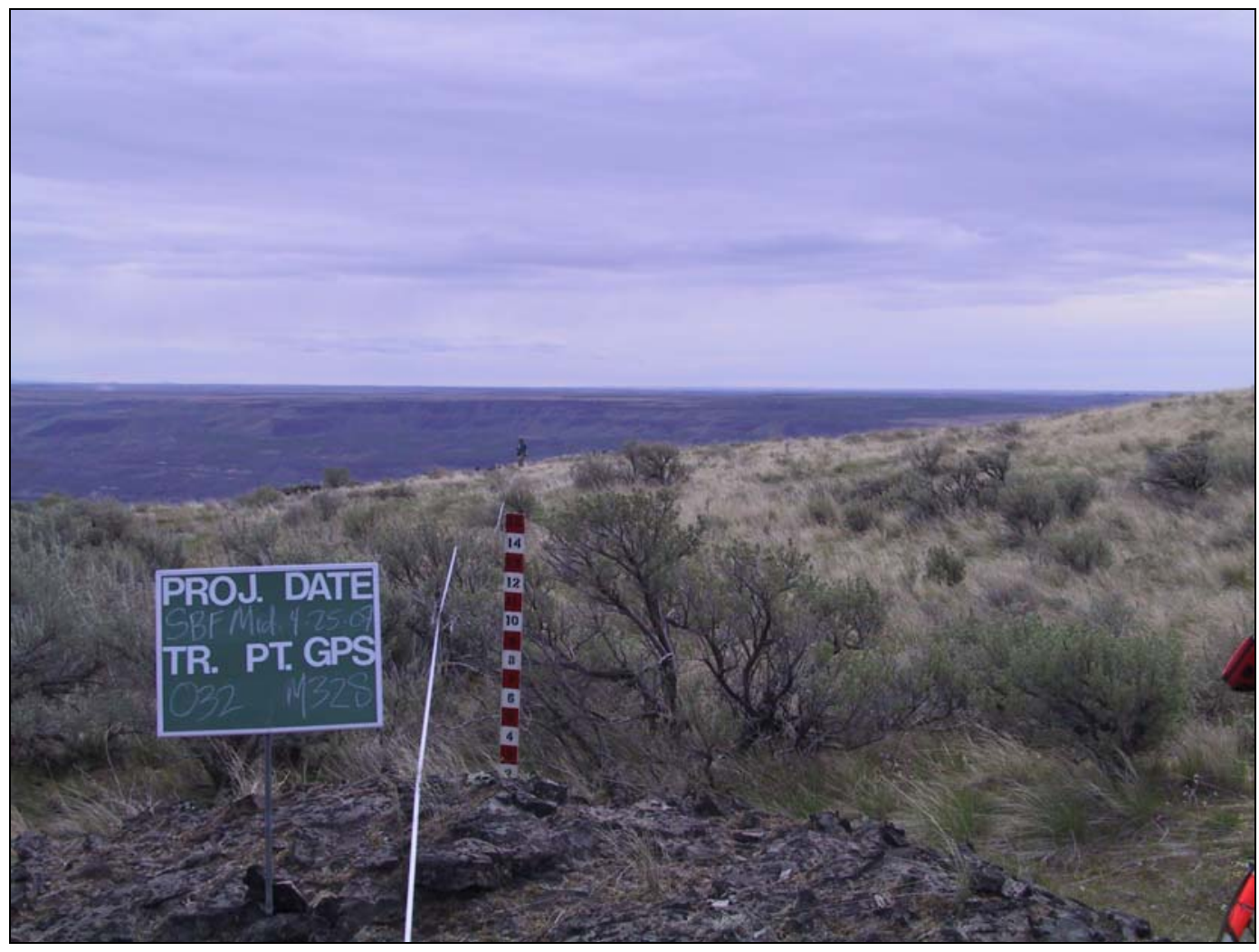

\section{Transect 34}

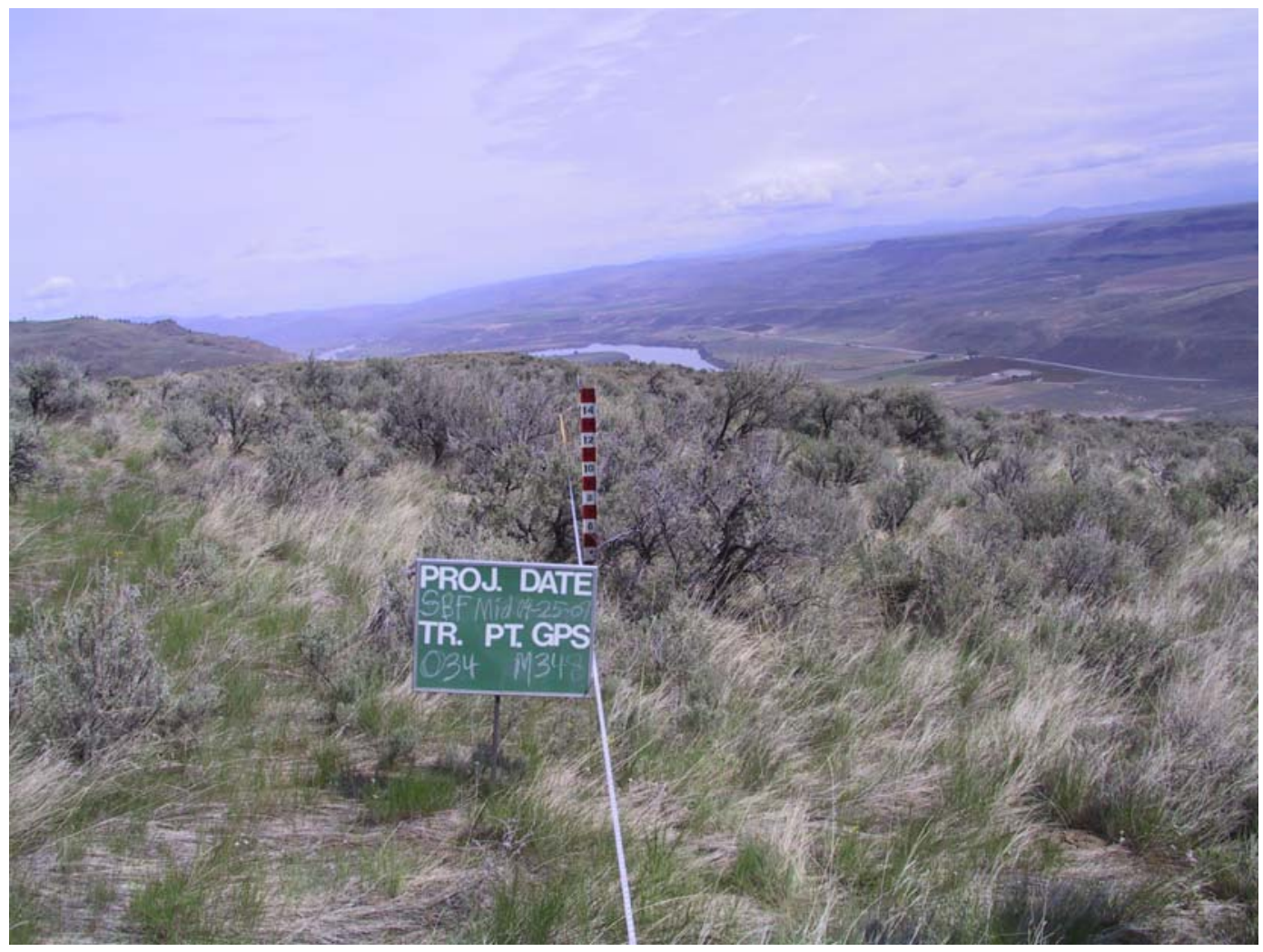


West Foster Creek Expansion Project

\section{Transect 35}

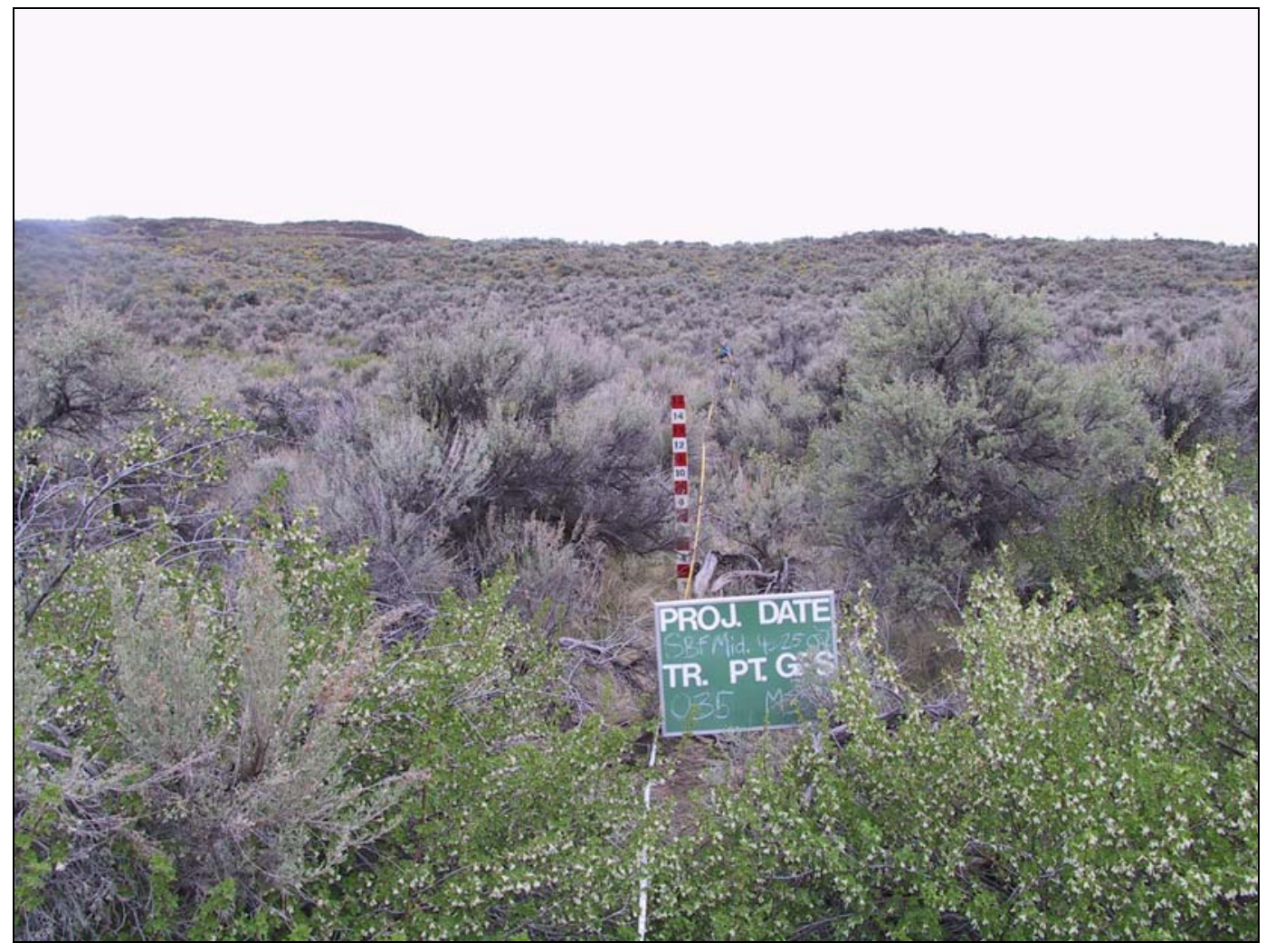

\section{Transect 36}

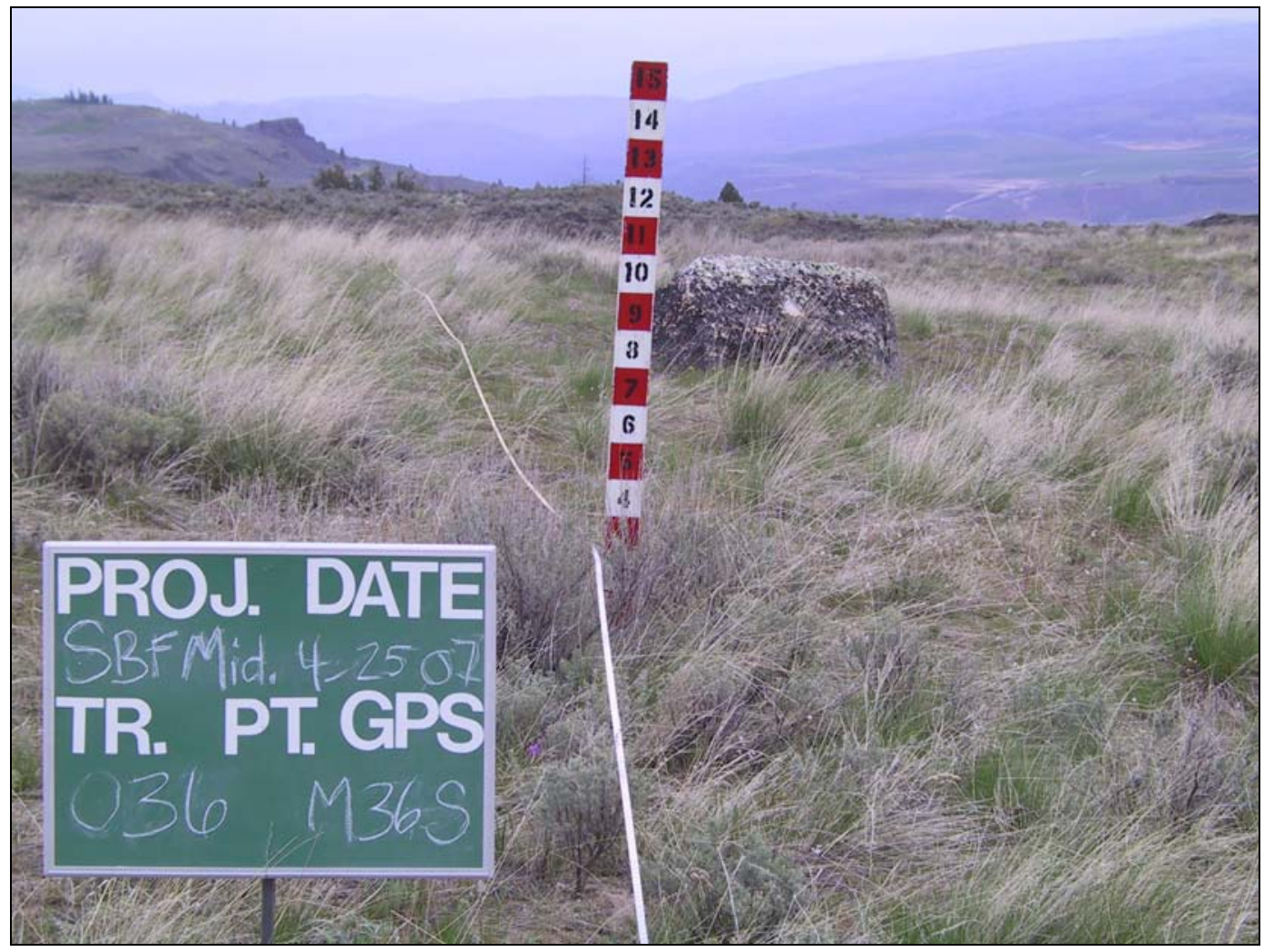


West Foster Creek Expansion Project

\section{Transect 37}

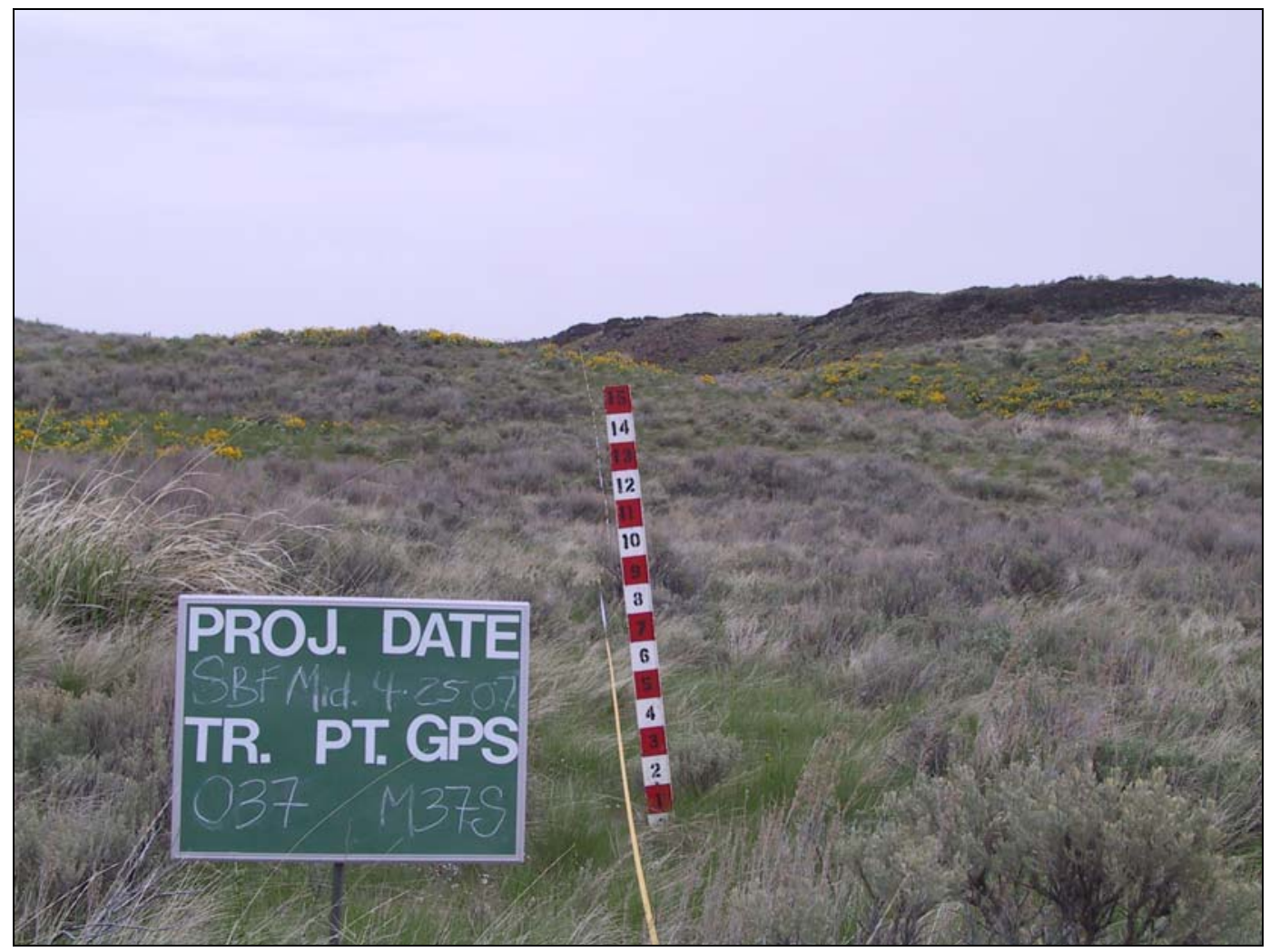

Transect 39

No photograph available 
West Foster Creek Expansion Project

Transect $\mathbf{4 0}$

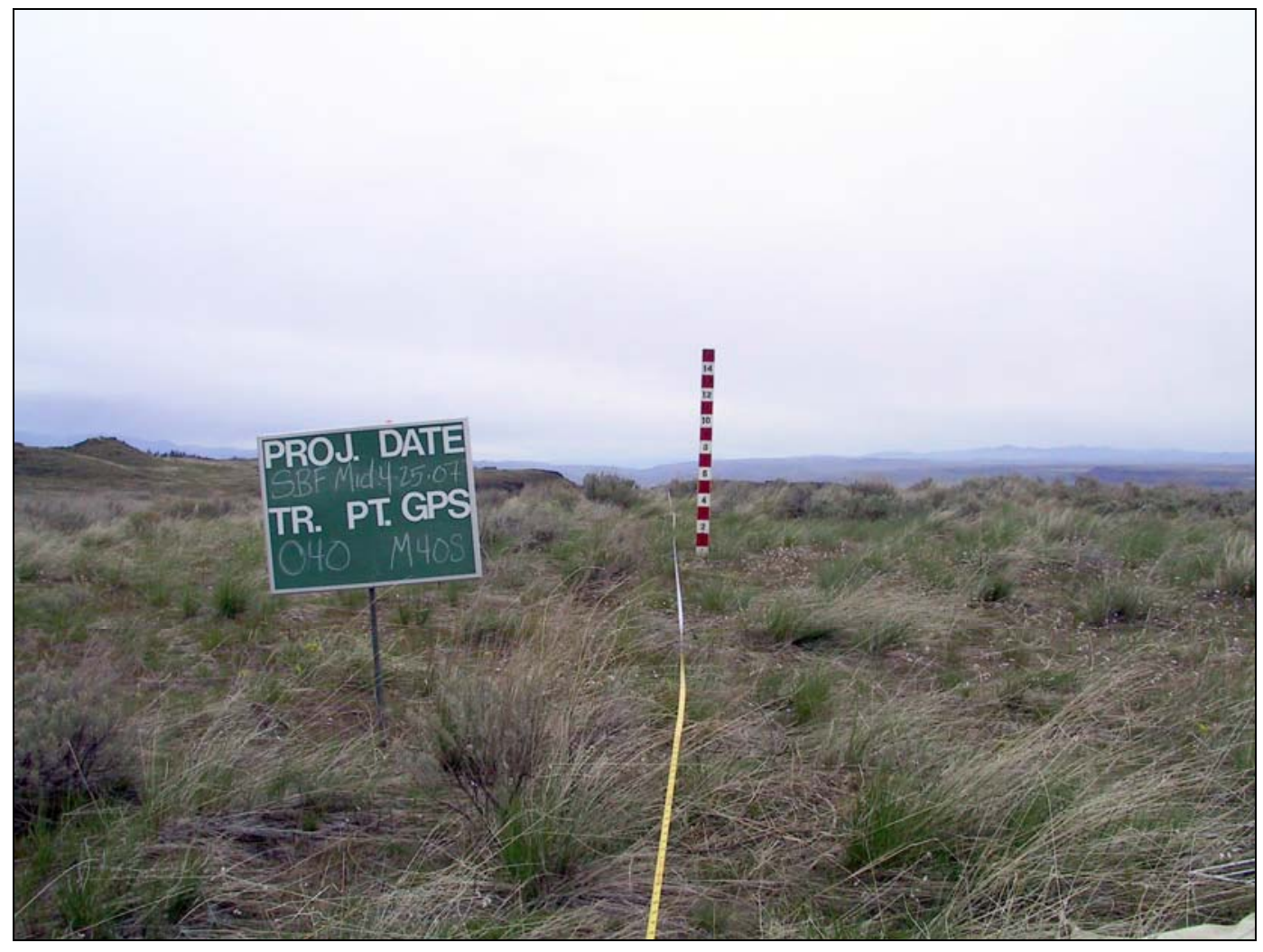


West Foster Creek Expansion Project

\section{McClain Lake}

\section{Transect 41}

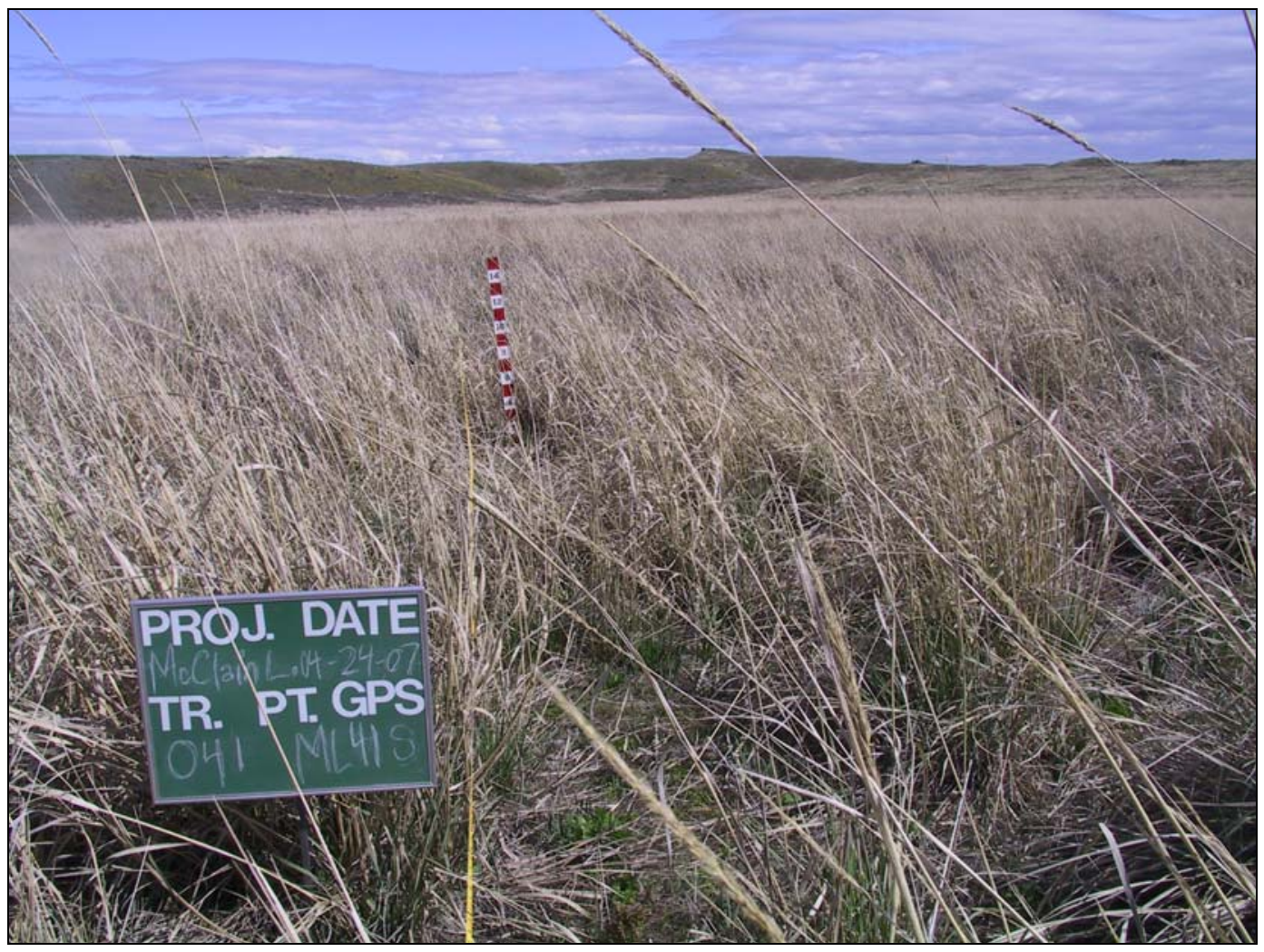


West Foster Creek Expansion Project

Transect 42

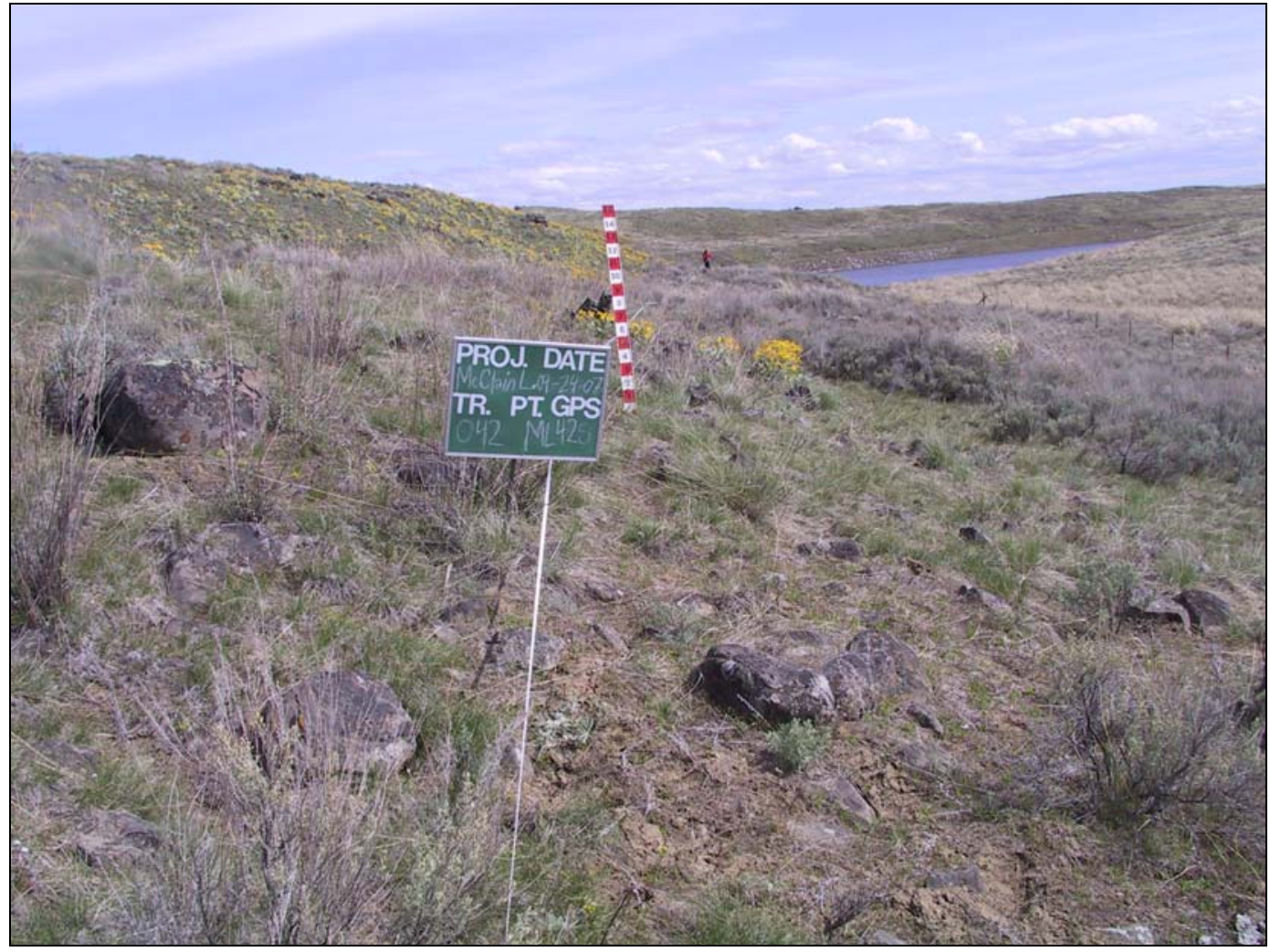

\section{Transect 43}

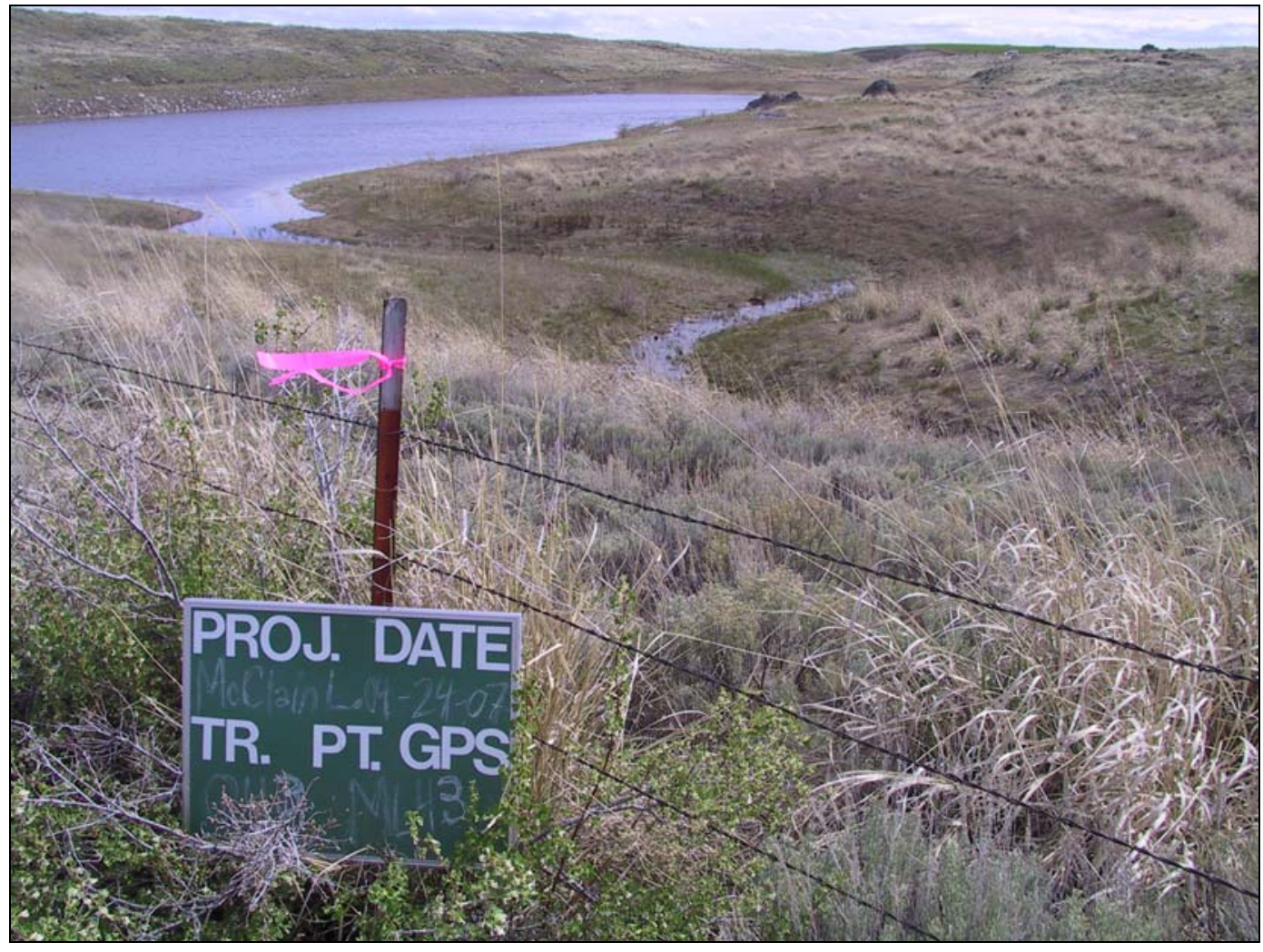


West Foster Creek Expansion Project

\section{Transect 45}

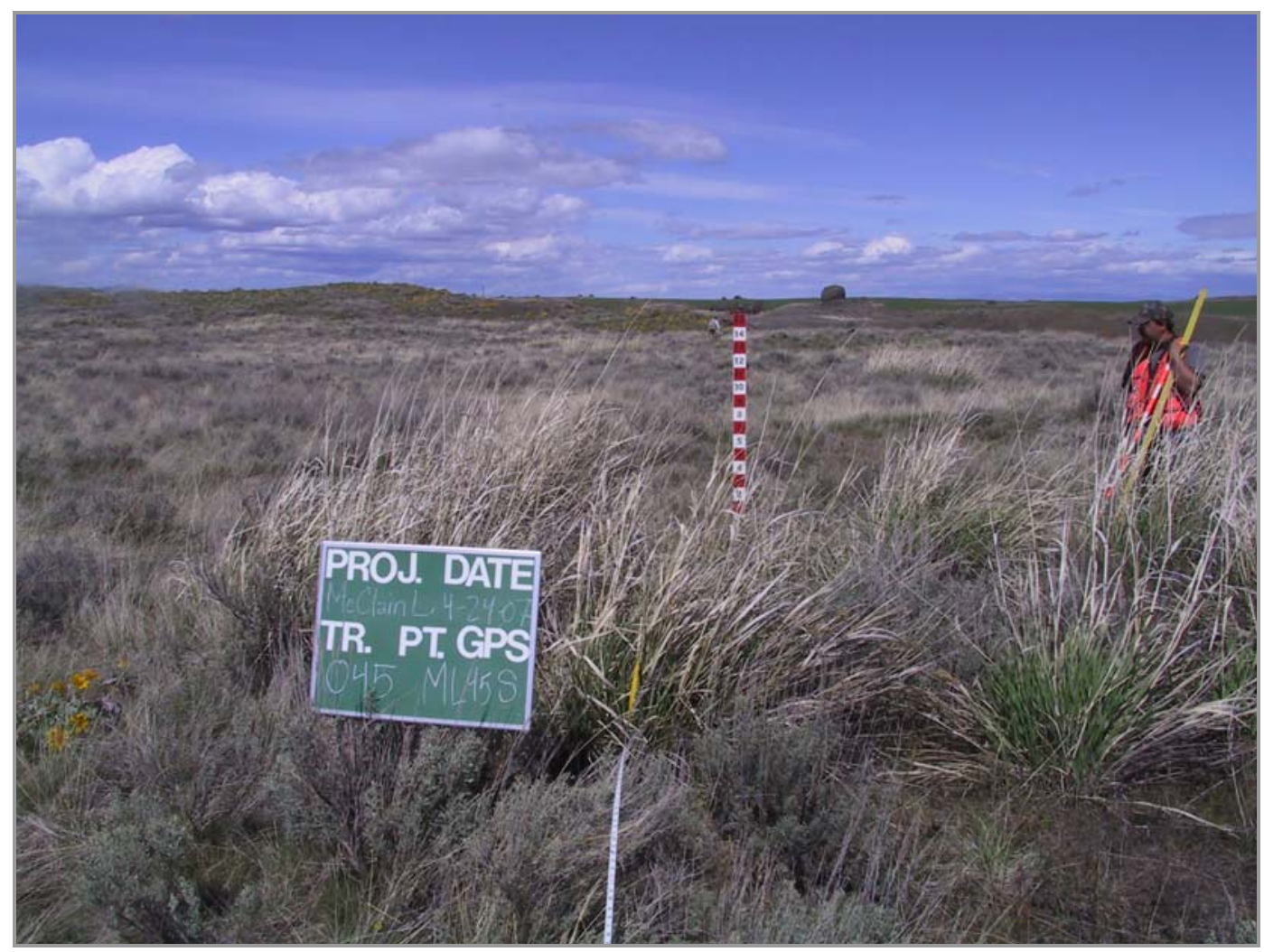

\section{Transect 46}

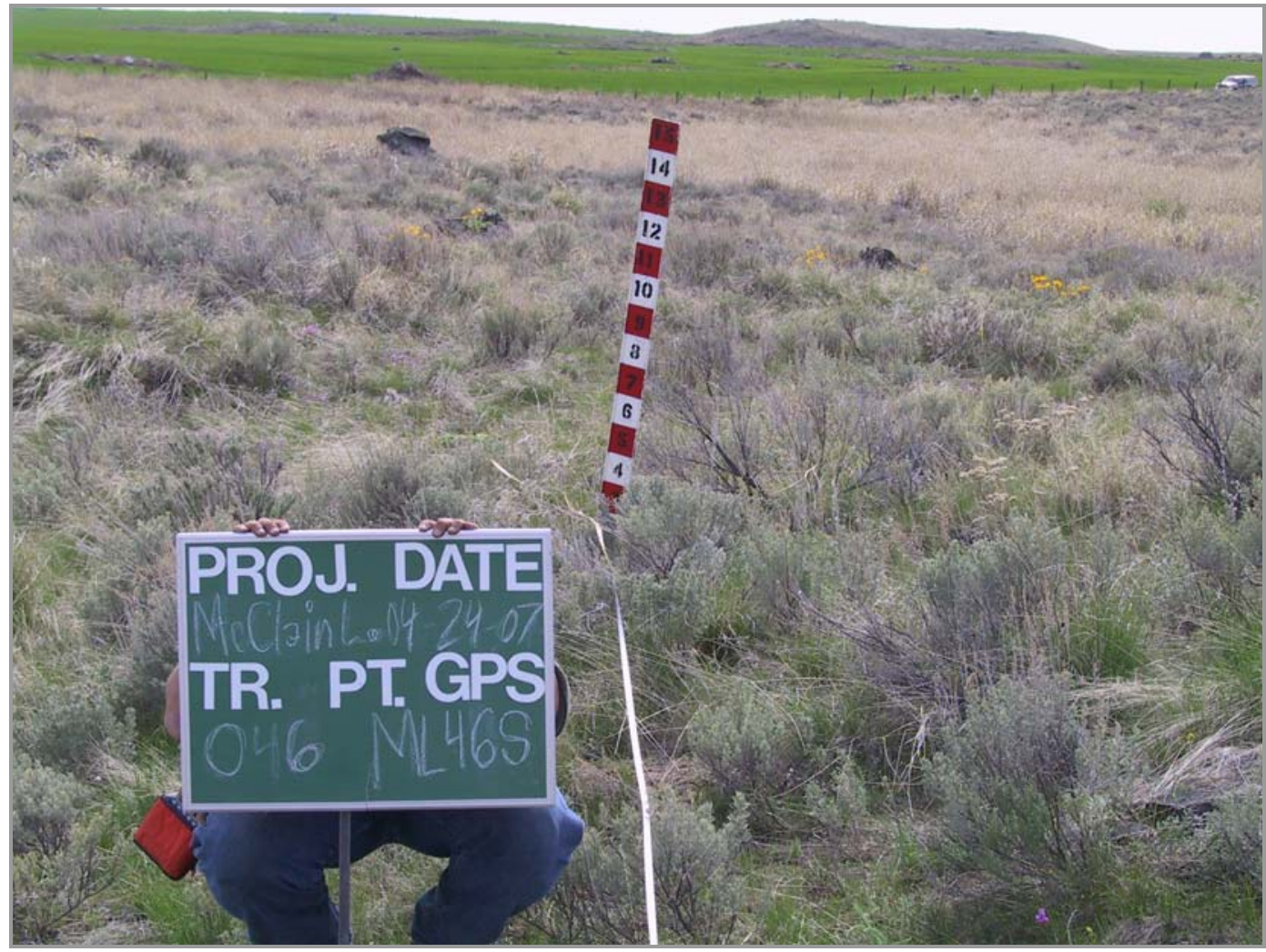


West Foster Creek Expansion Project

\section{Transect 47}

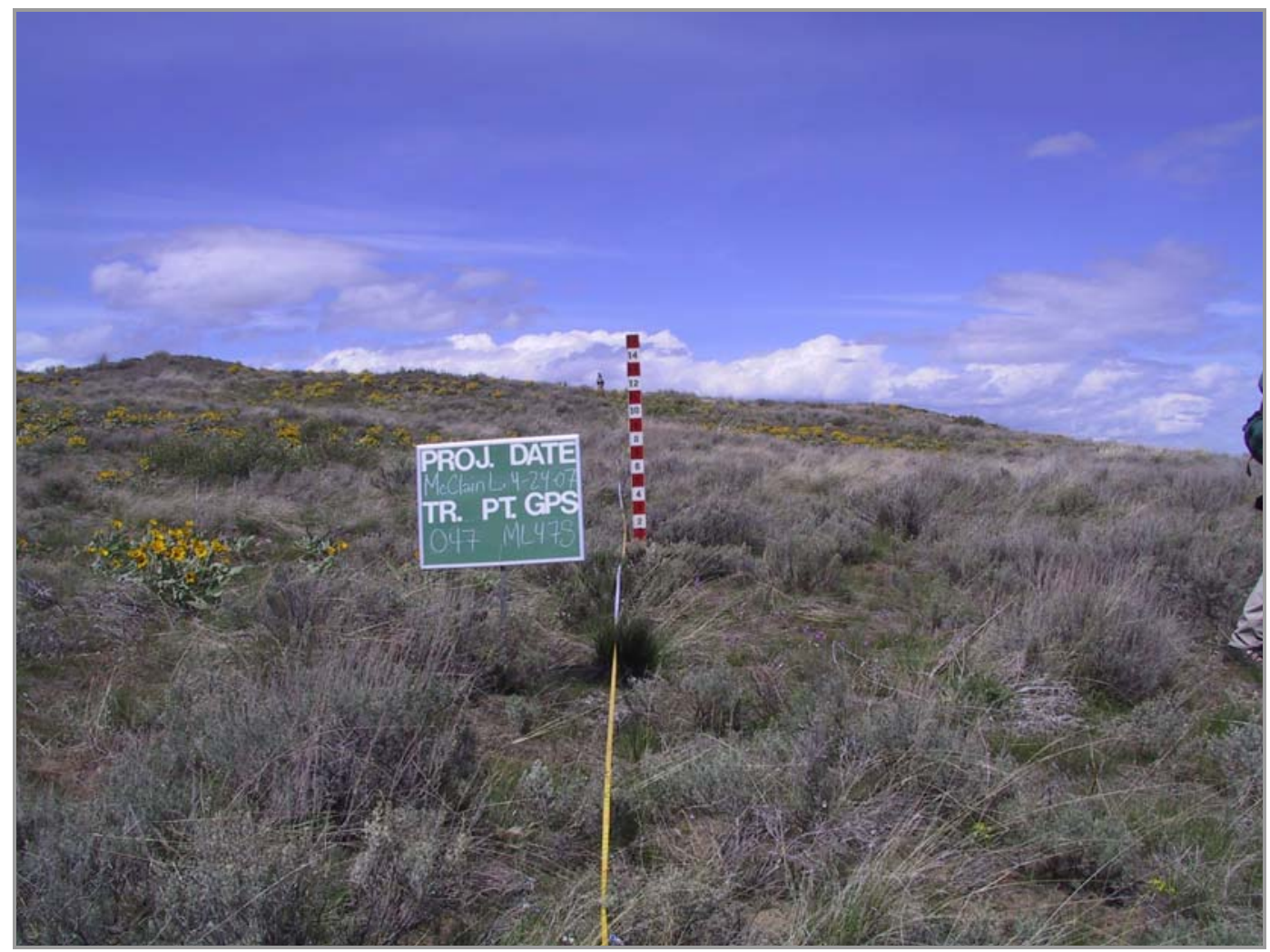


West Foster Creek Expansion Project

Transect 49

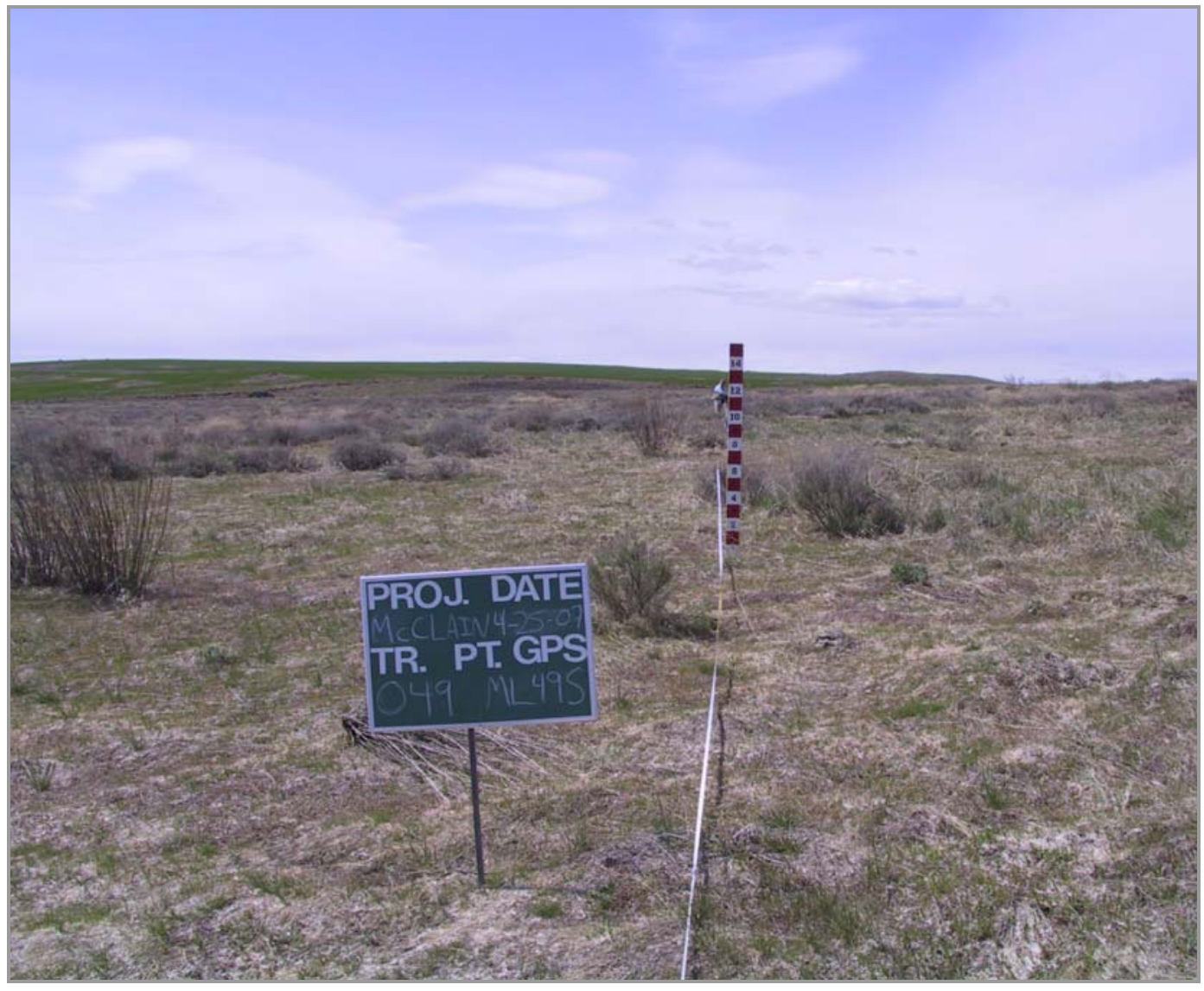

\section{Transect 51}

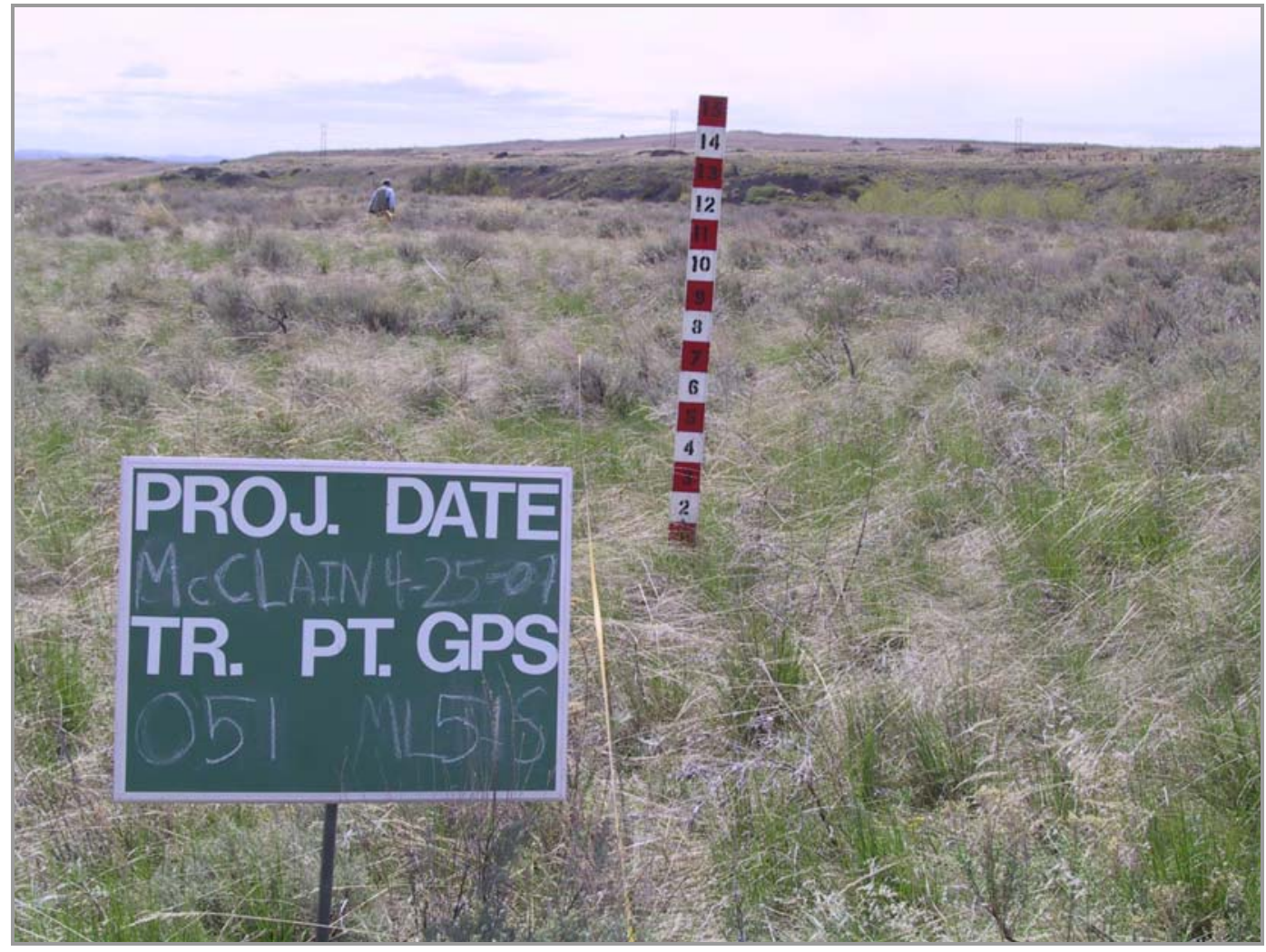


West Foster Creek Expansion Project

\section{Transect 52}

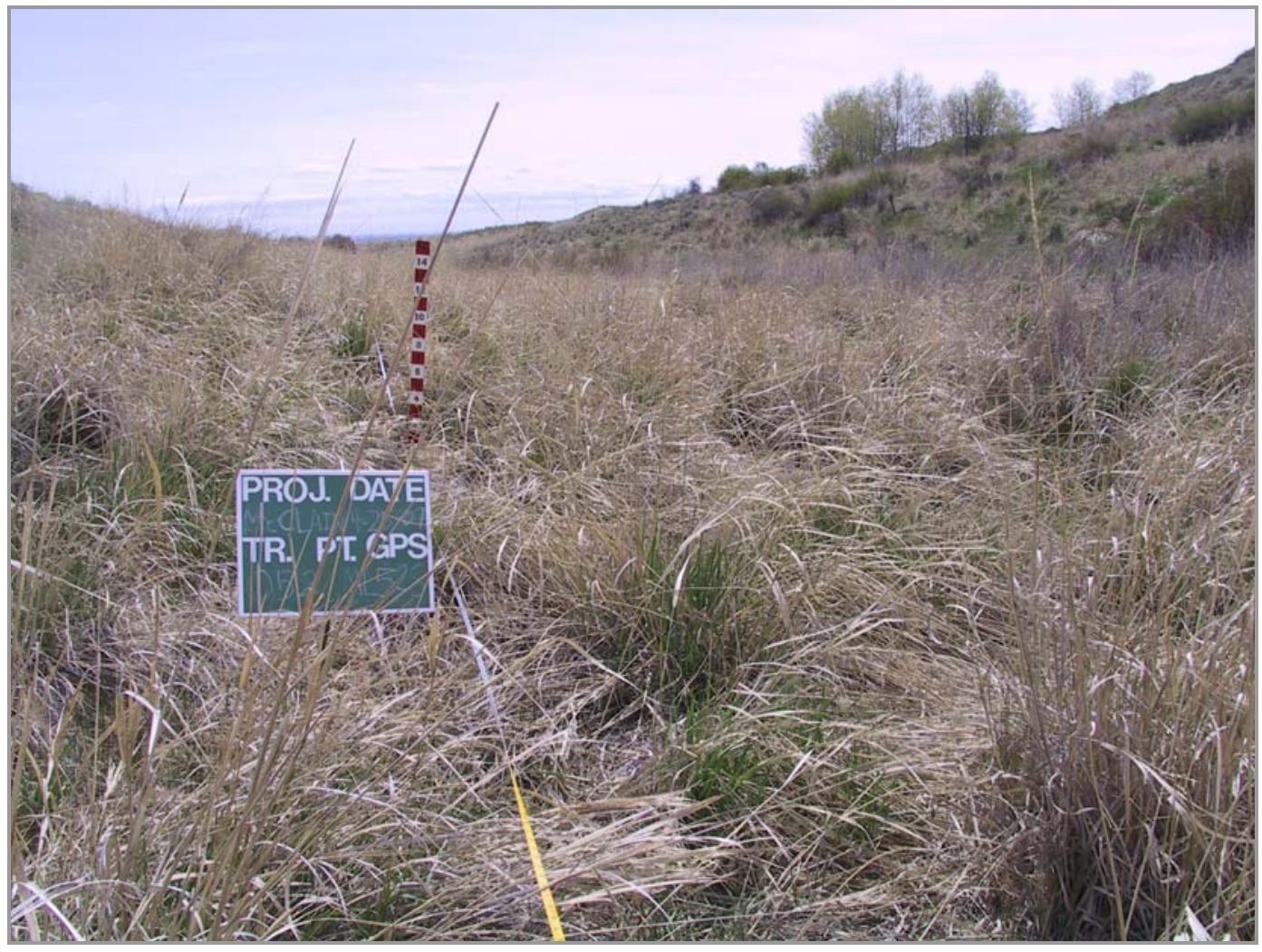

\section{Transect 57}

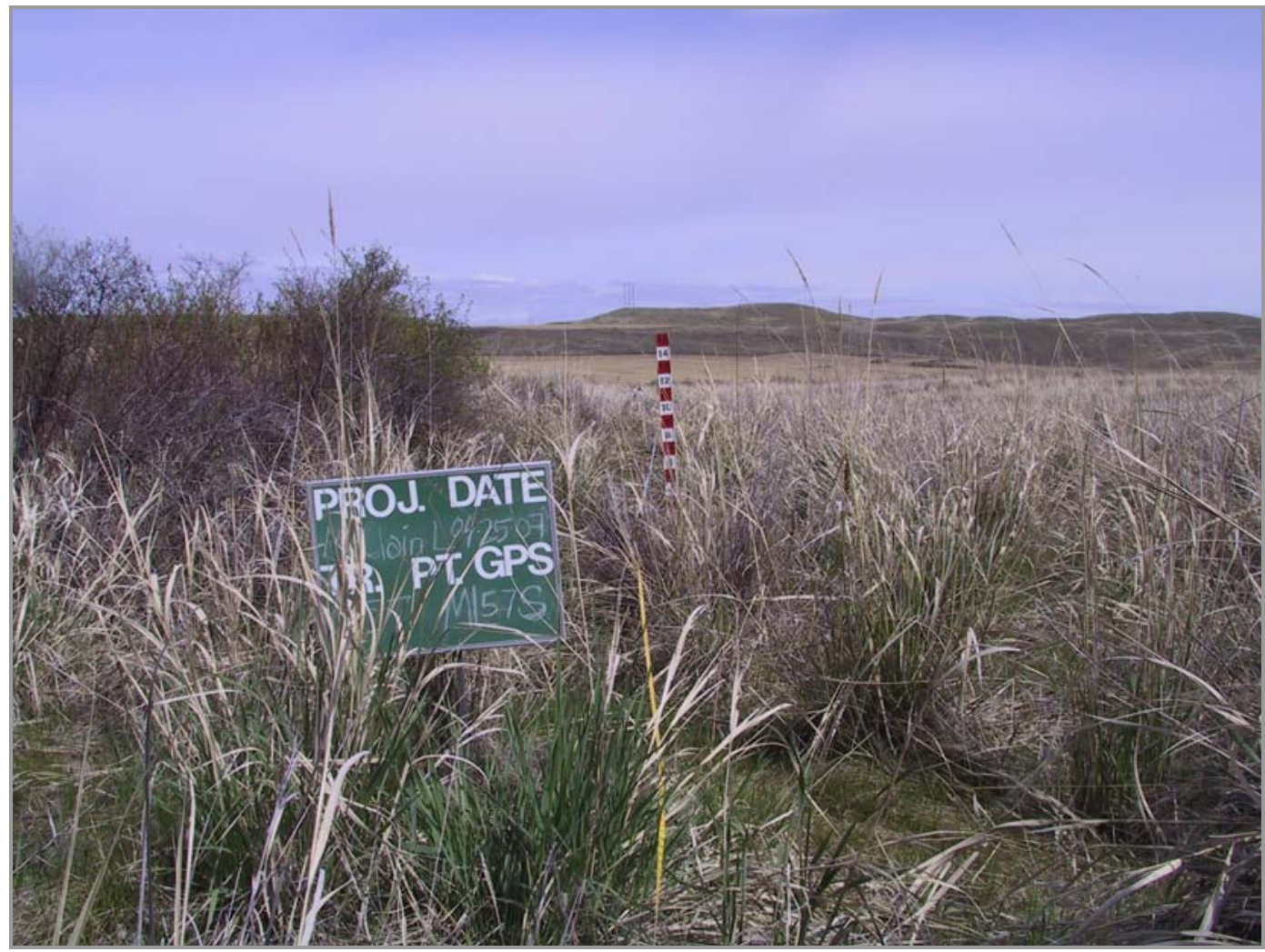


West Foster Creek Expansion Project

\section{Transect 59}

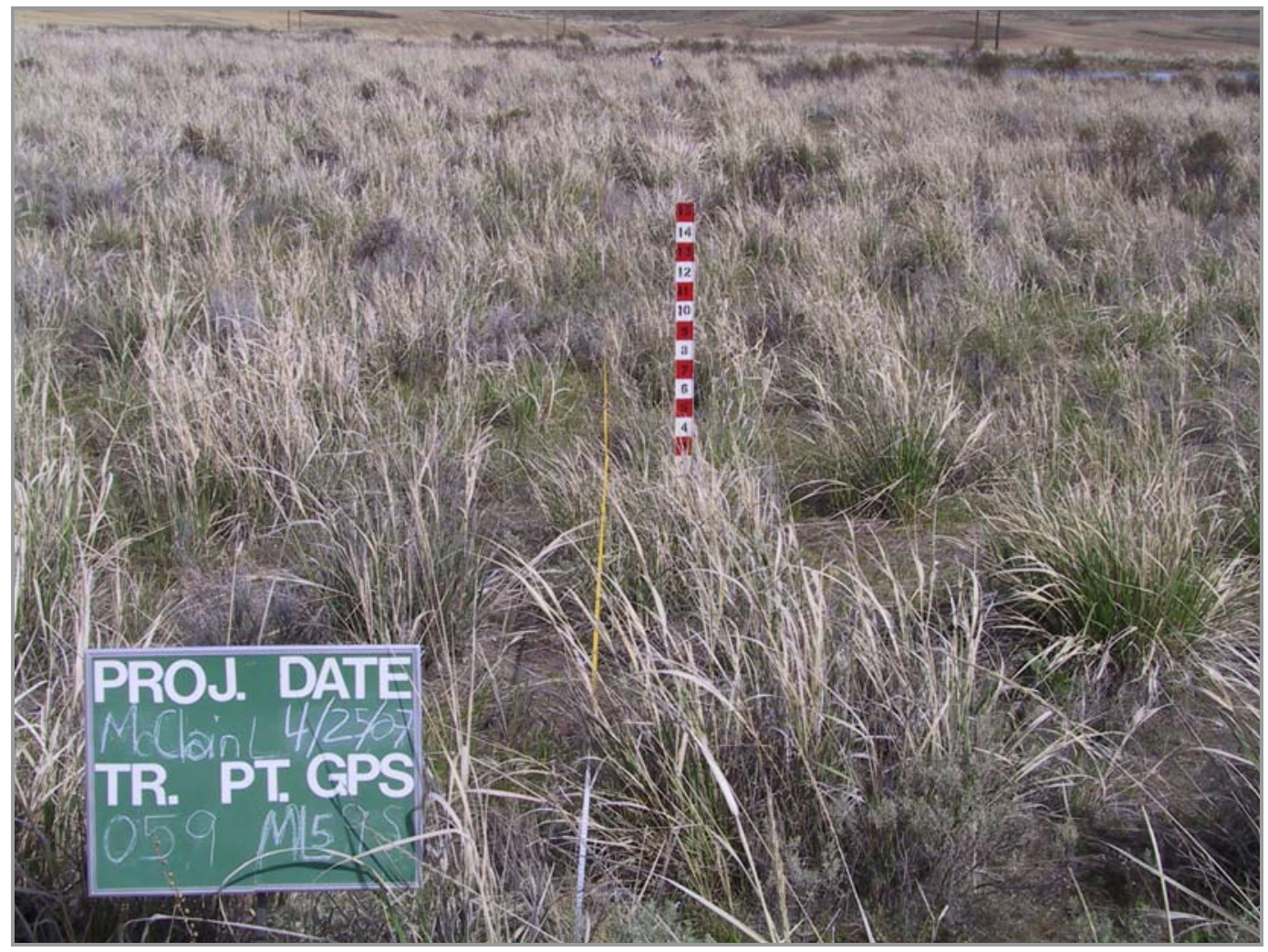

\section{Transect 70}

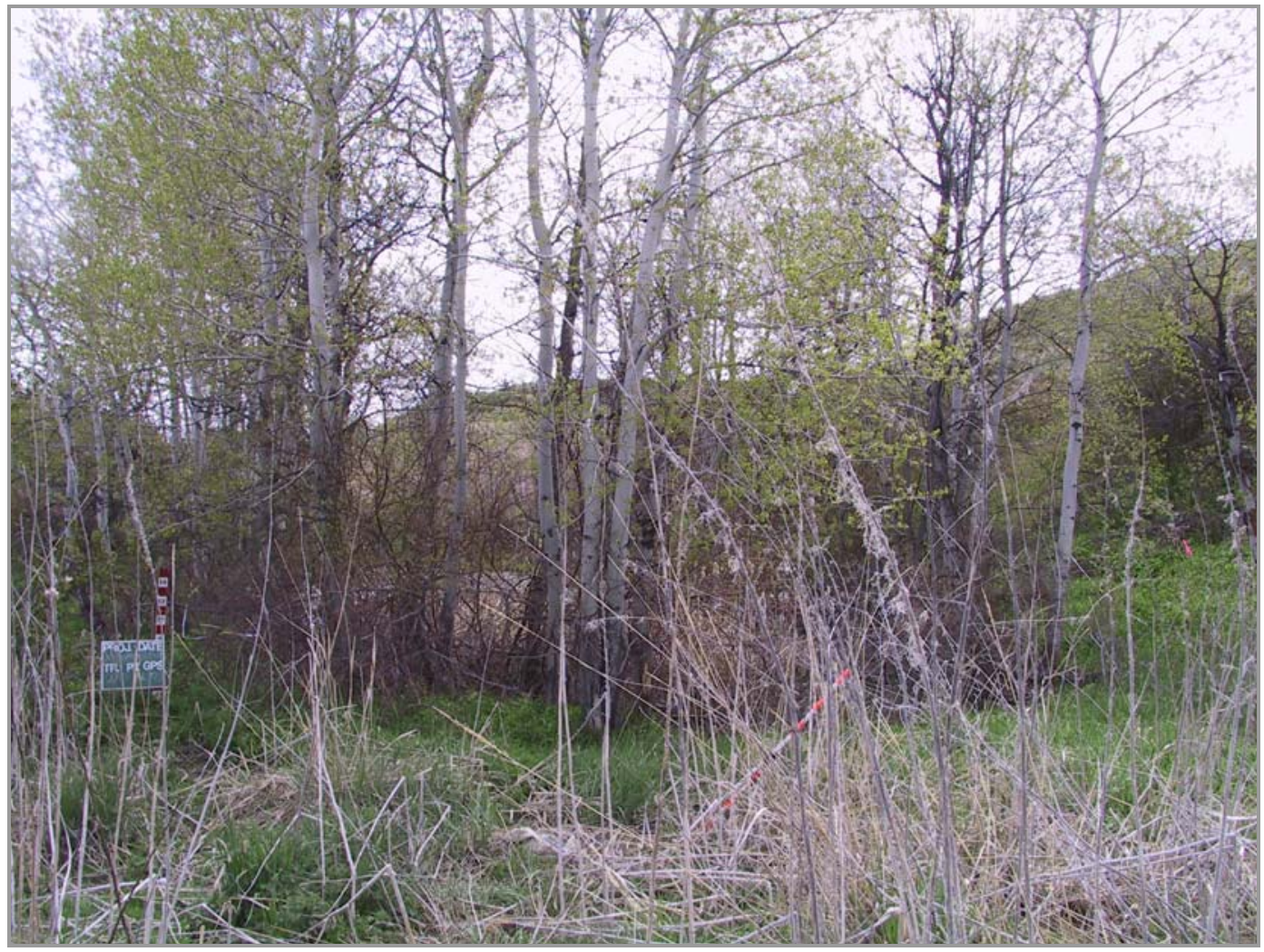


West Foster Creek Expansion Project

\section{Transect 71}

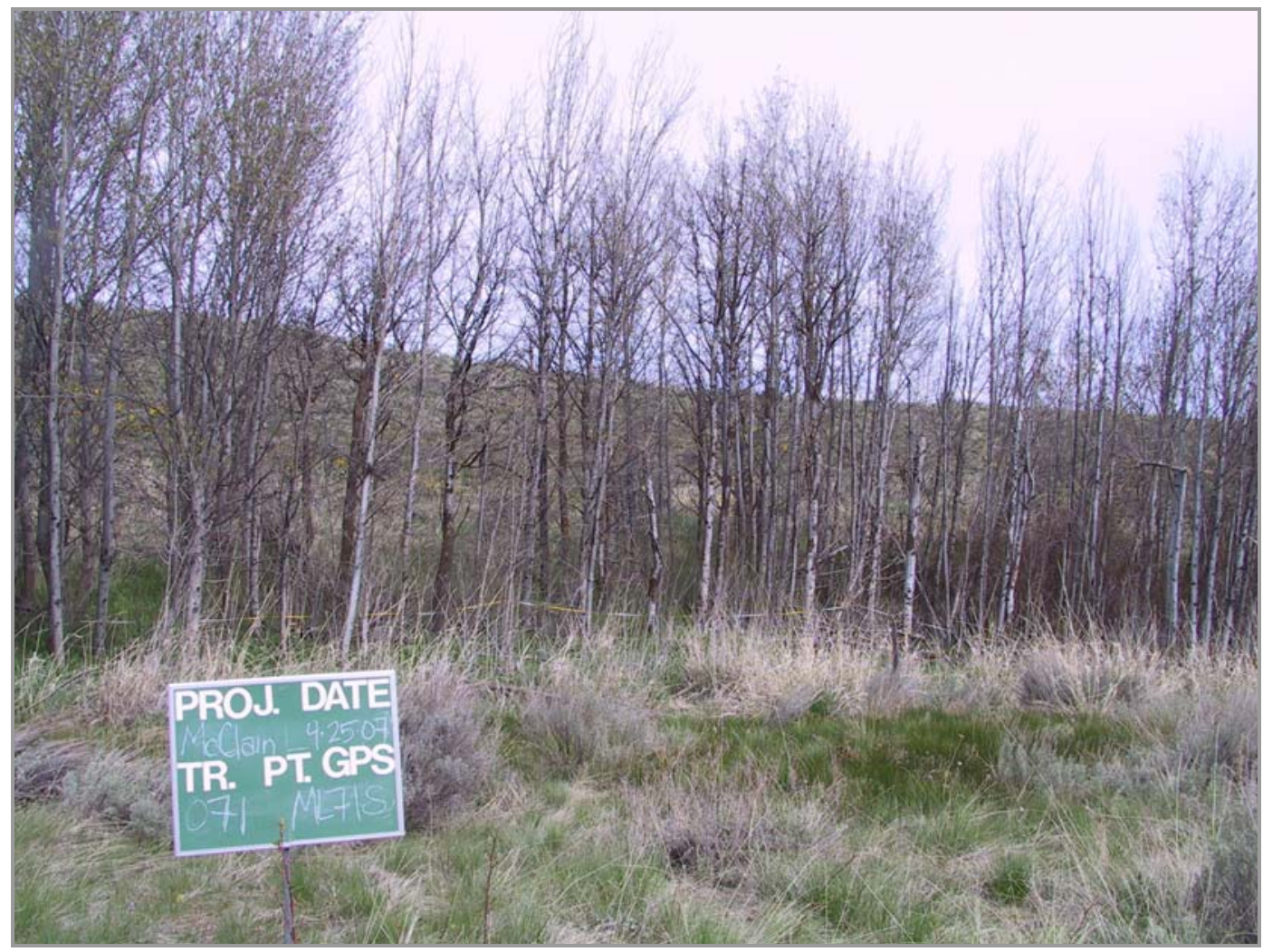

Transect 72

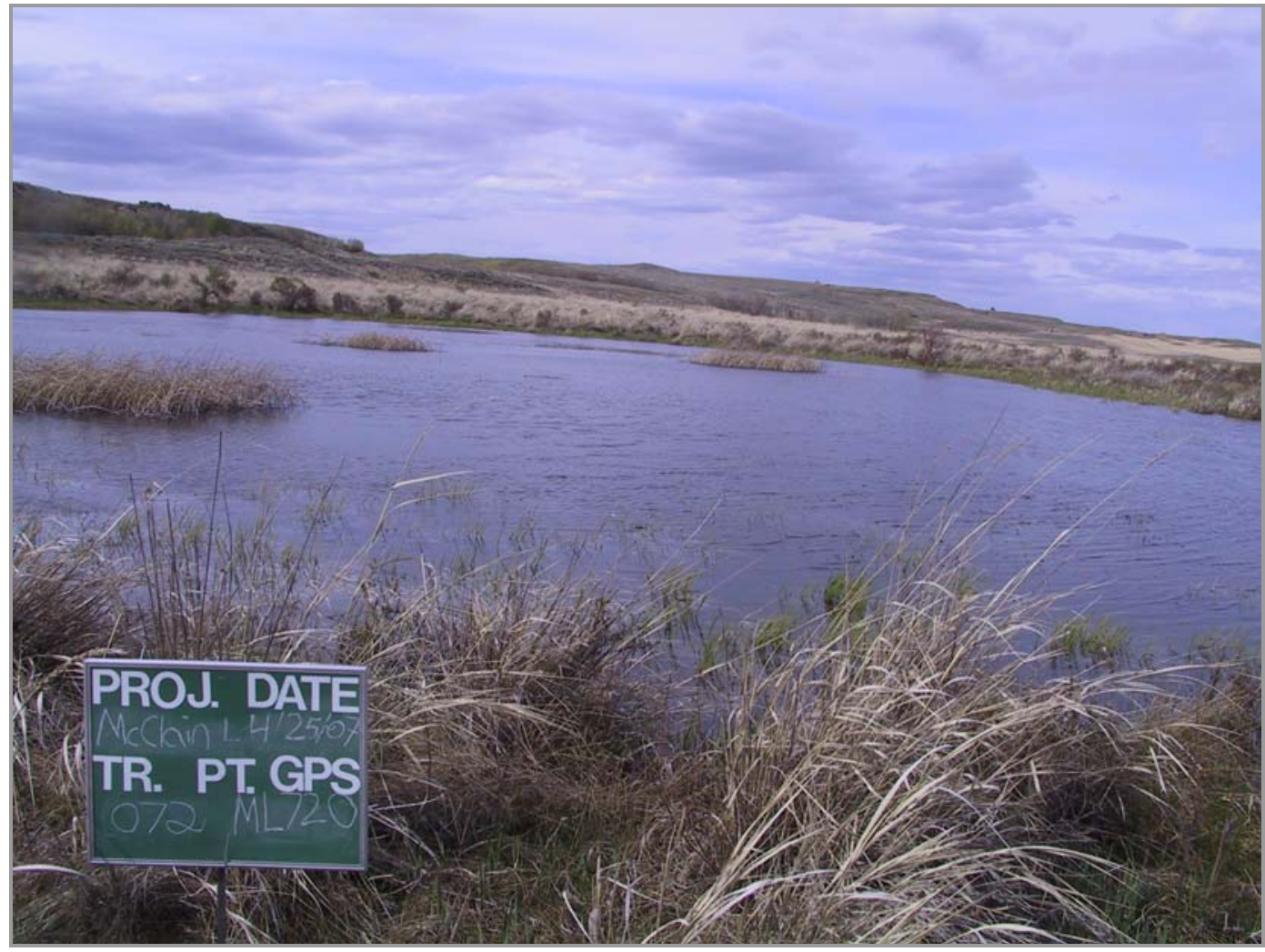


West Foster Creek Expansion Project

\section{Dezellum Lake}

\section{Transect 2}

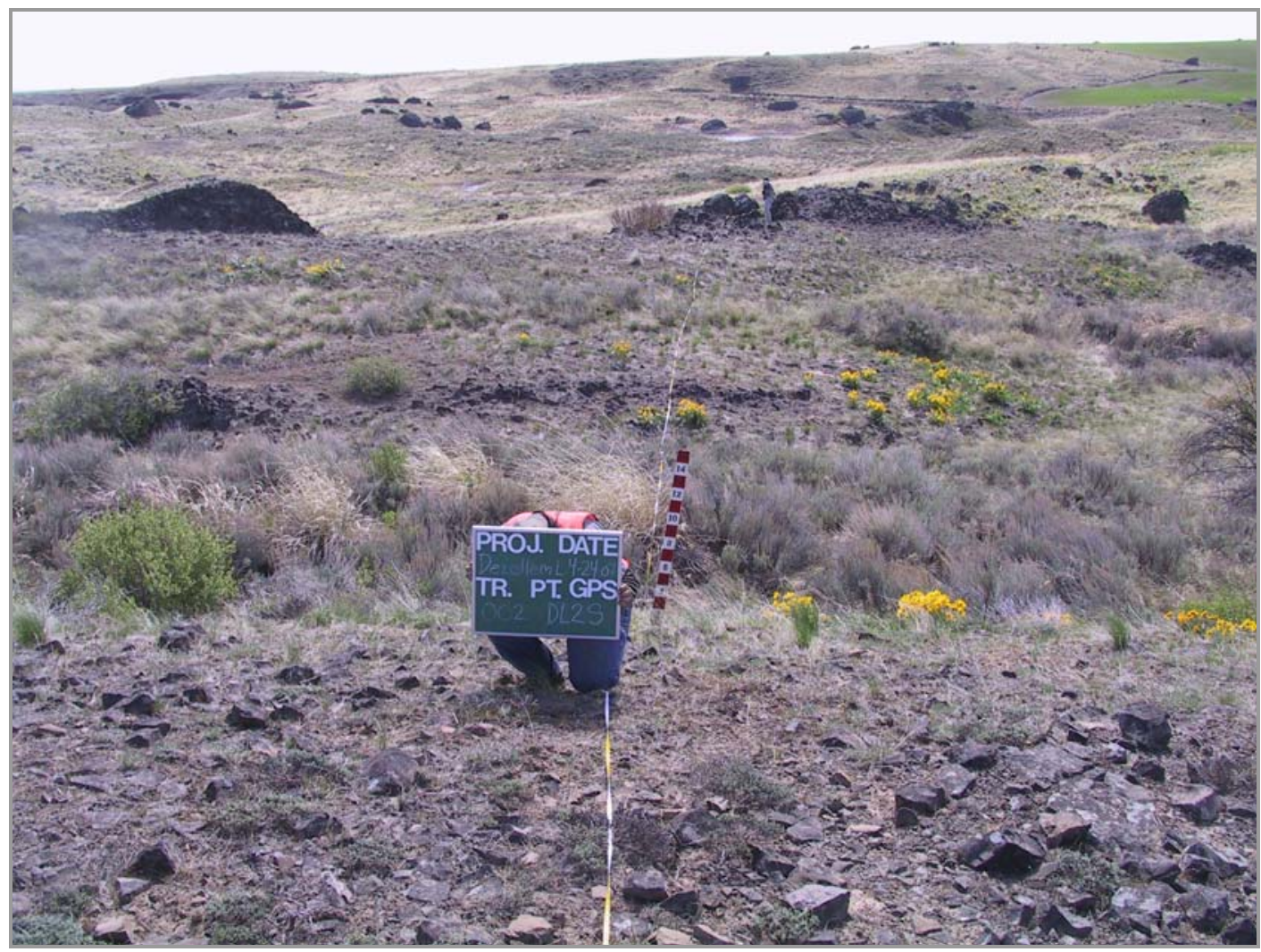


West Foster Creek Expansion Project

Transect 3

Transect 4

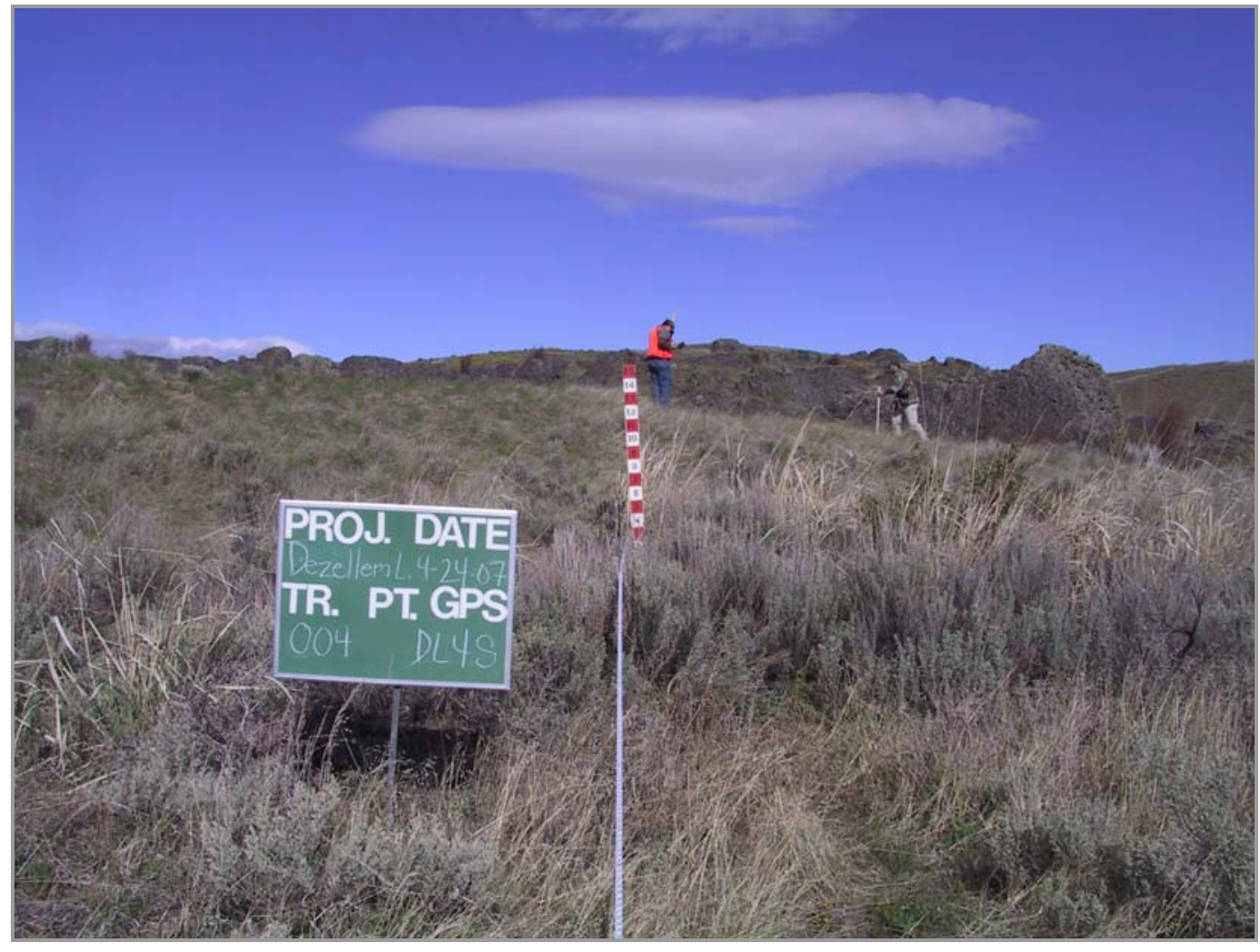


West Foster Creek Expansion Project

\section{Transect 6}

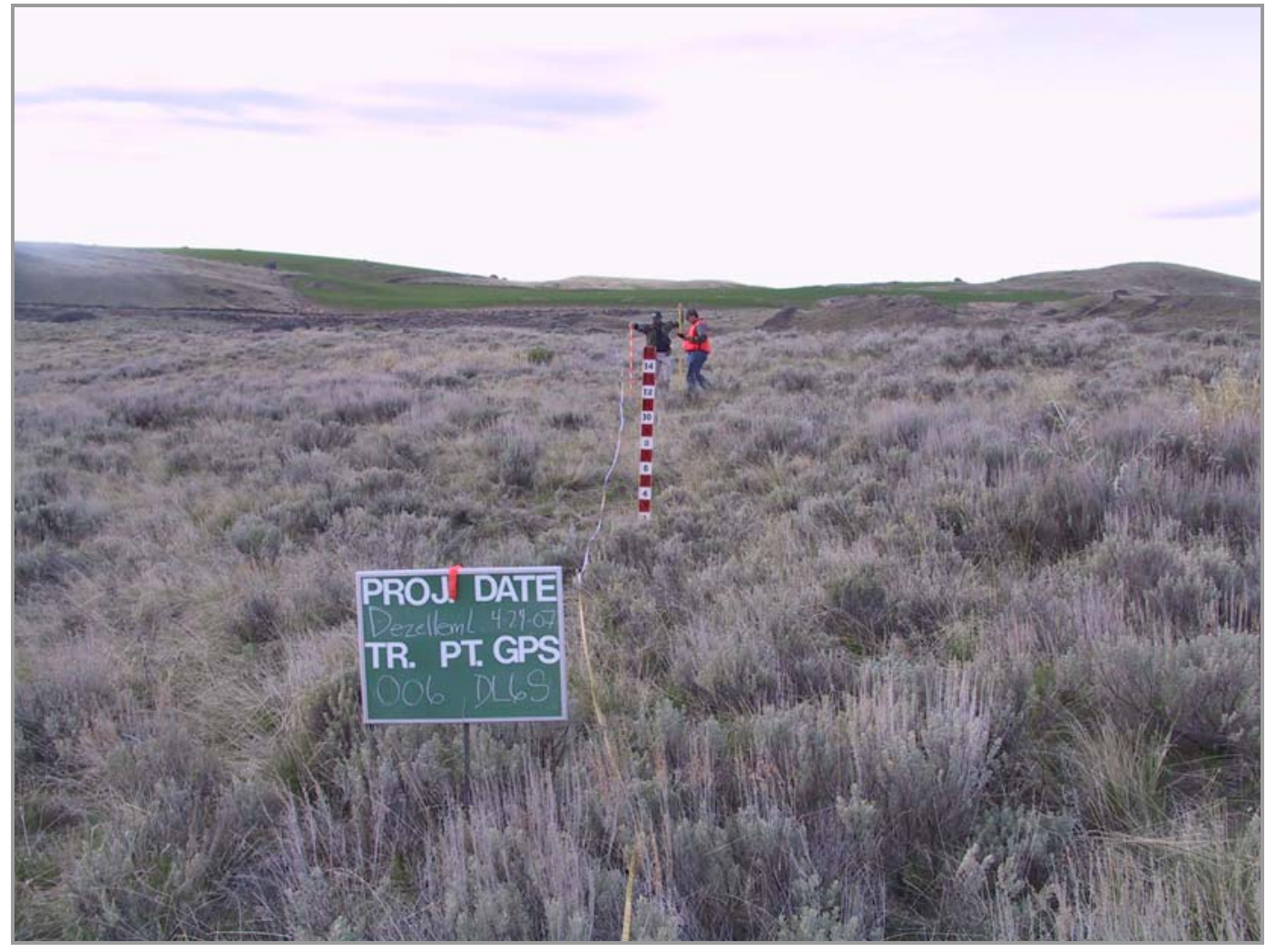

\section{Transect 7}

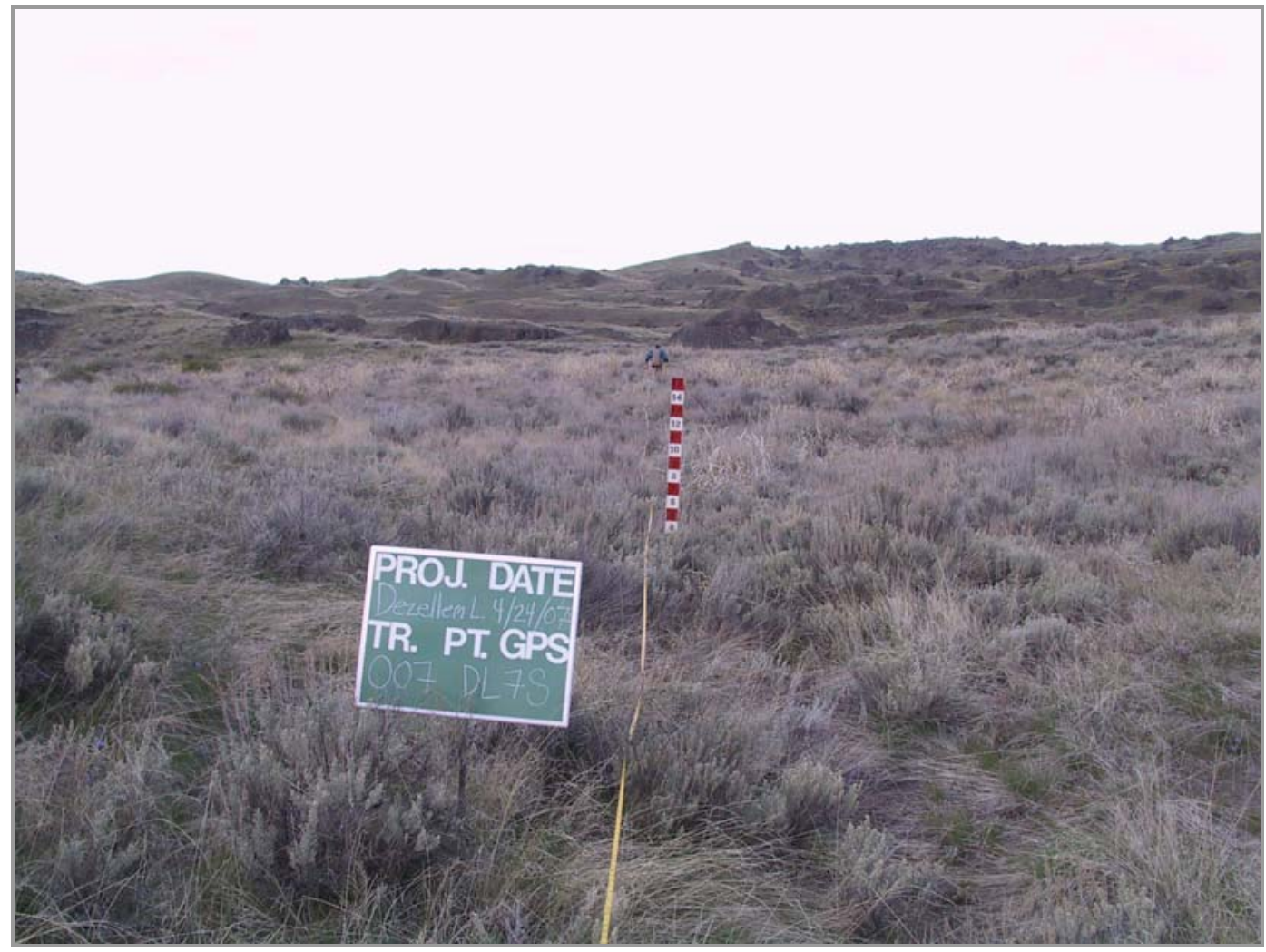


West Foster Creek Expansion Project

Transect 10

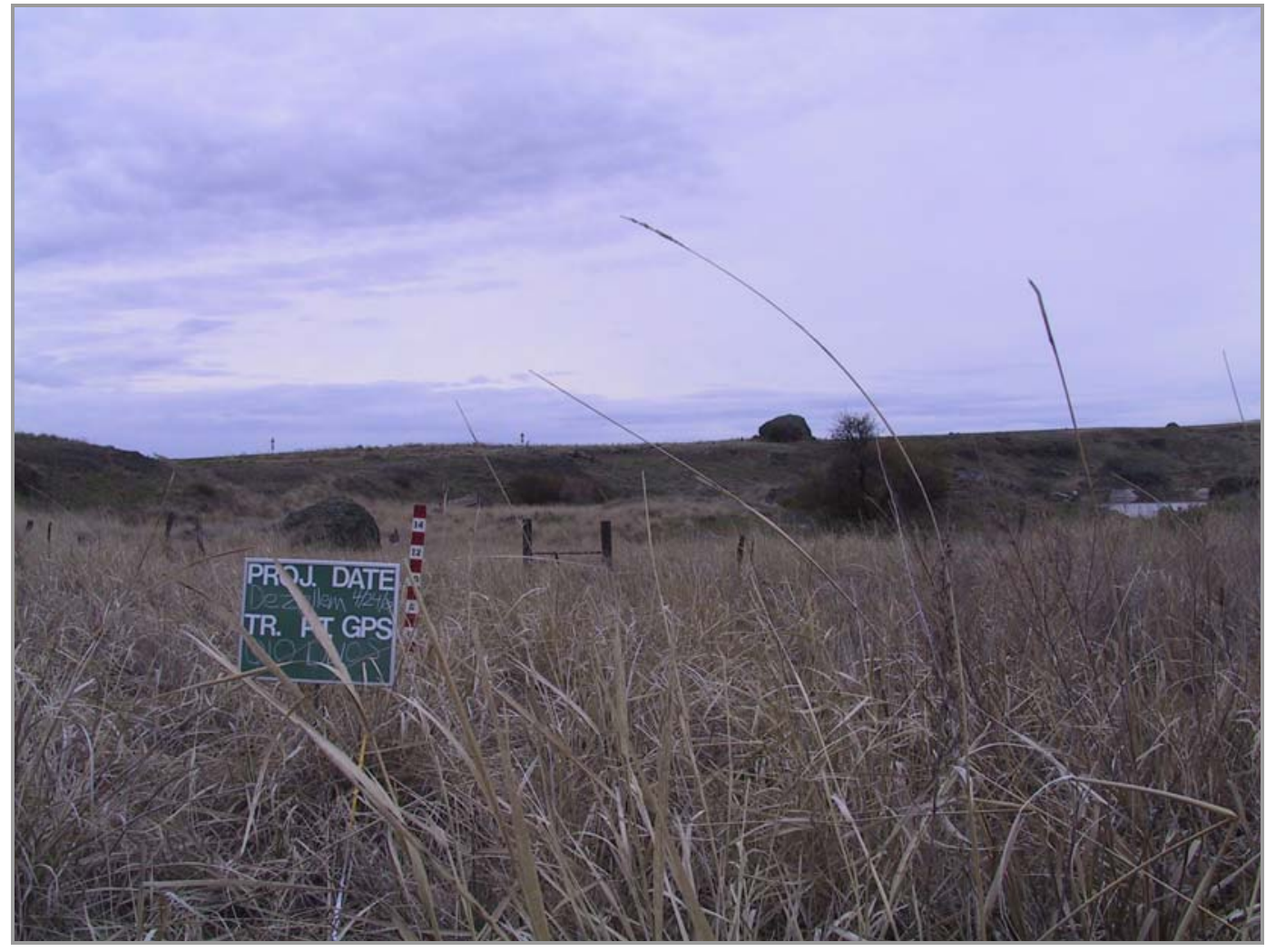

Transect 13

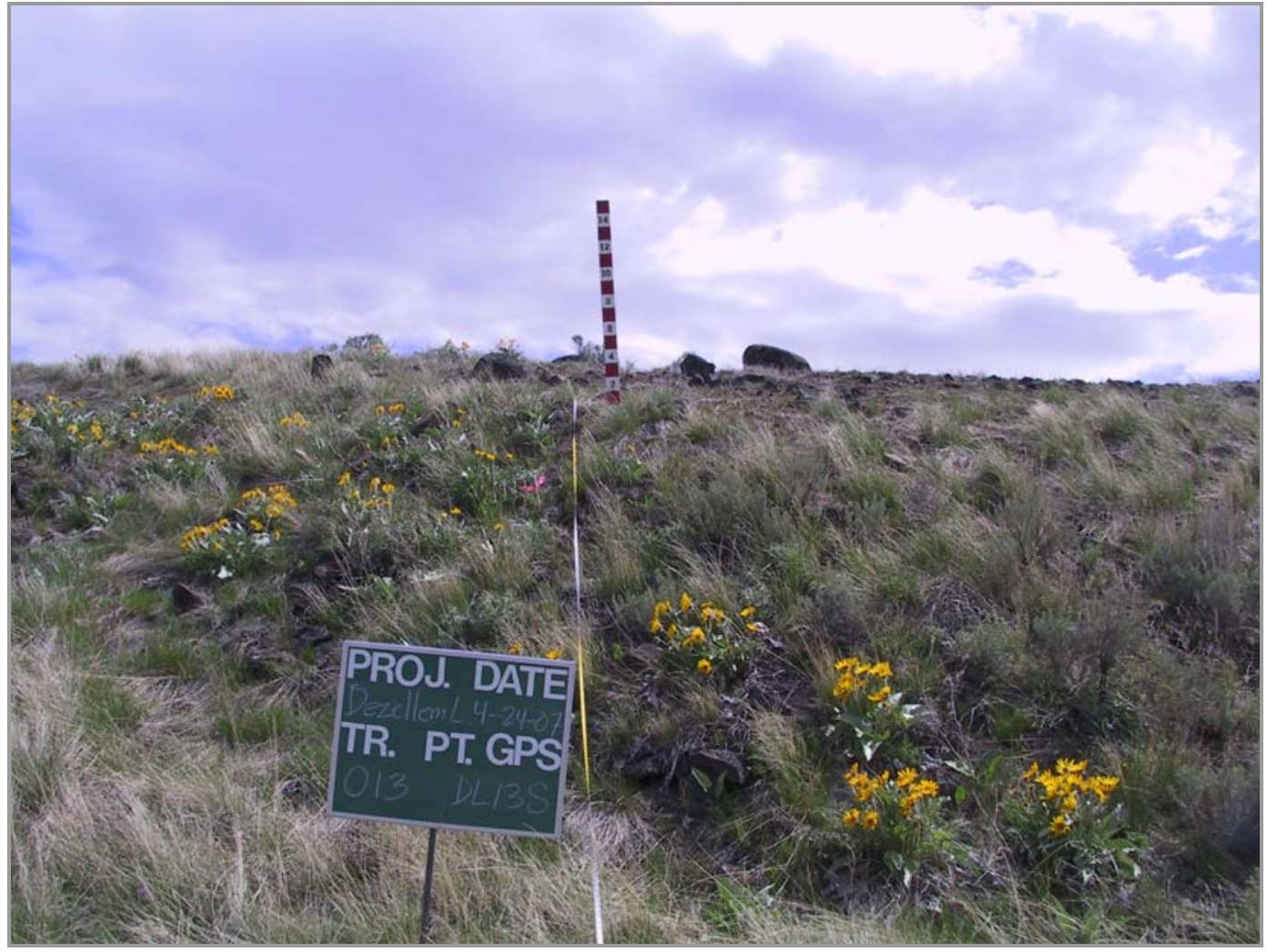


West Foster Creek Expansion Project

\section{Transect 14}

No photograph available

\section{Transect 17}

No photograph available

\section{Transect 22}

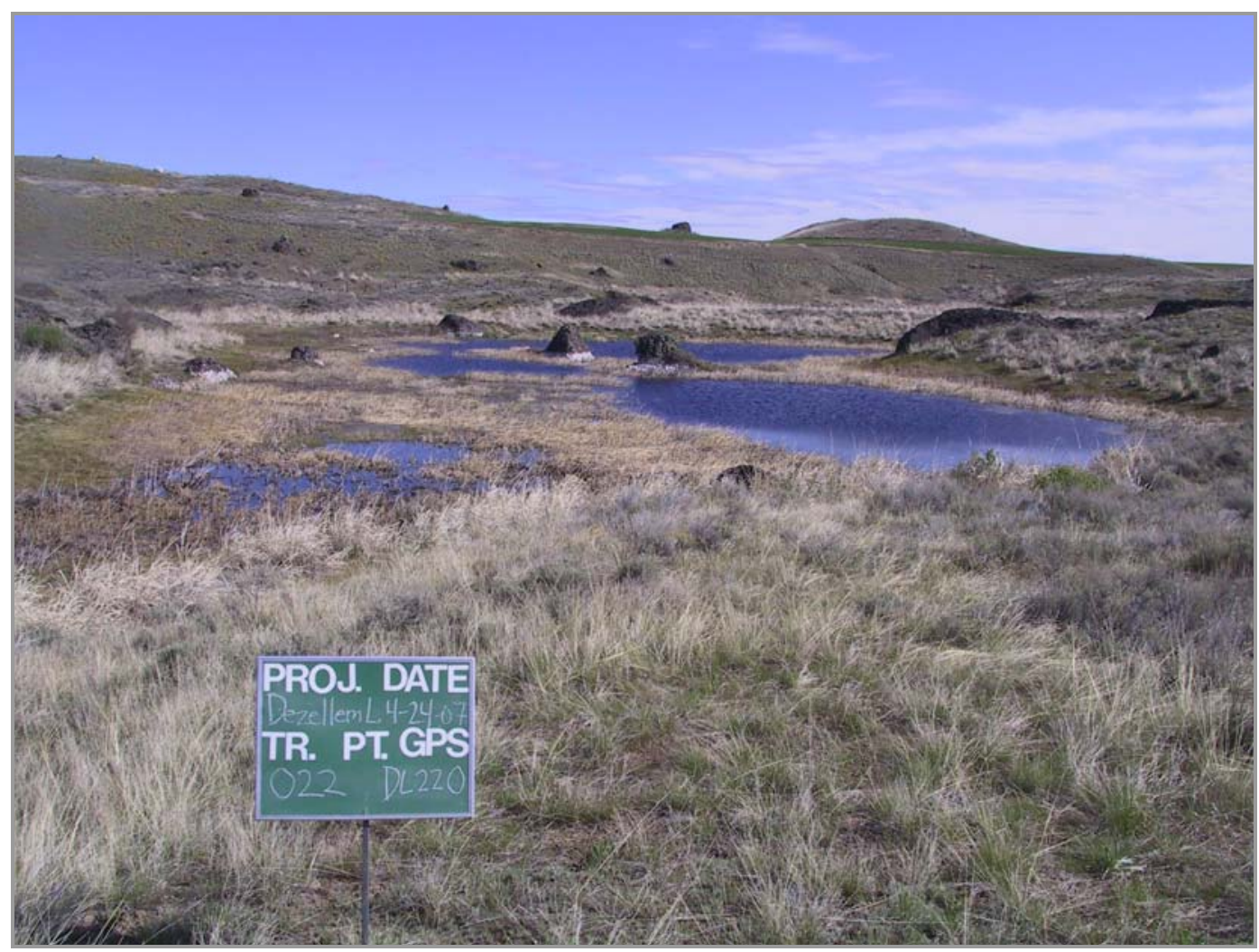


West Foster Creek Expansion Project

Transect 23

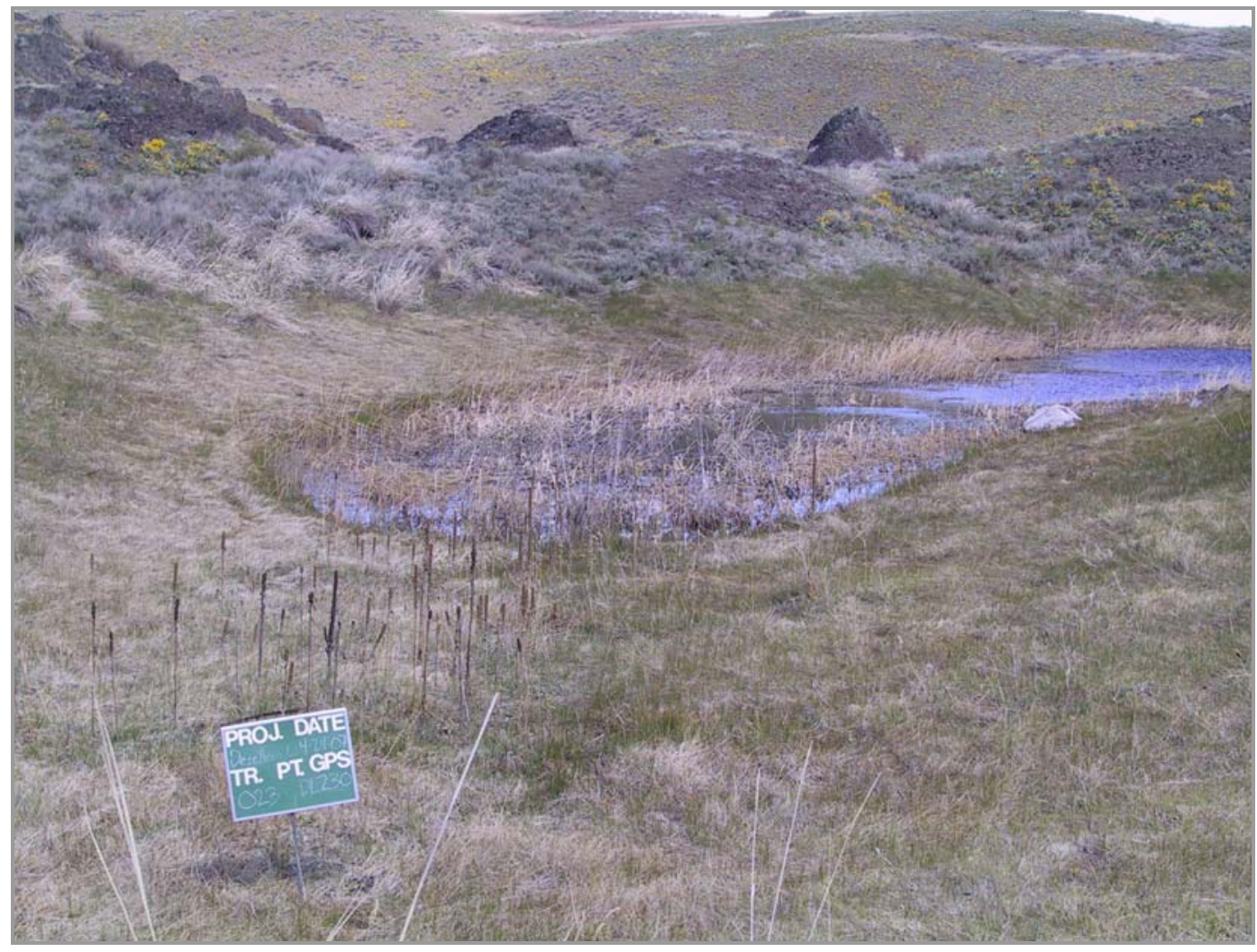

Transect 24

No Photograph Available

\section{Transect 25}

No Photograph Available 
West Foster Creek Expansion Project

\section{JoJaCo}

\section{Transect 20}

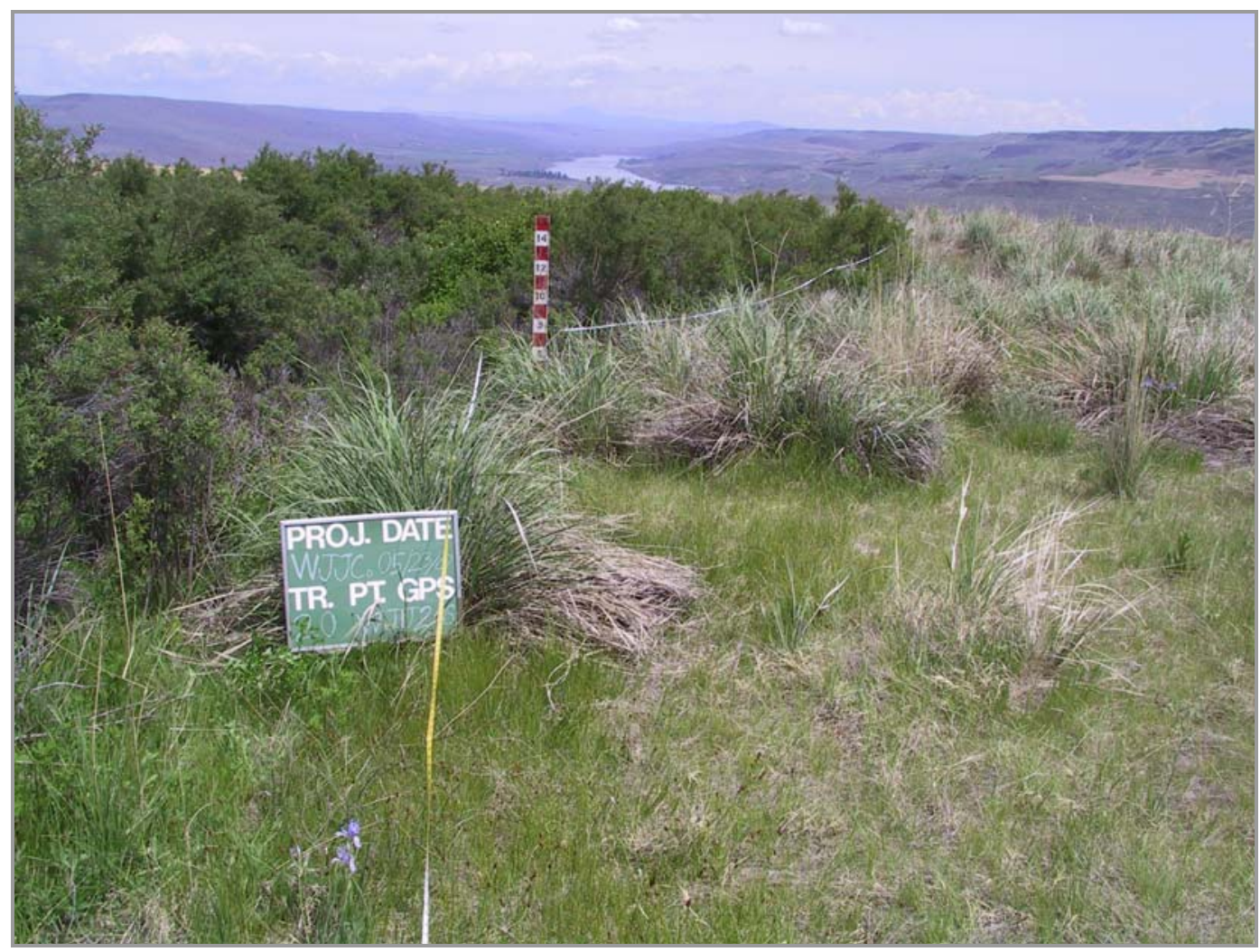


West Foster Creek Expansion Project

Transect 21

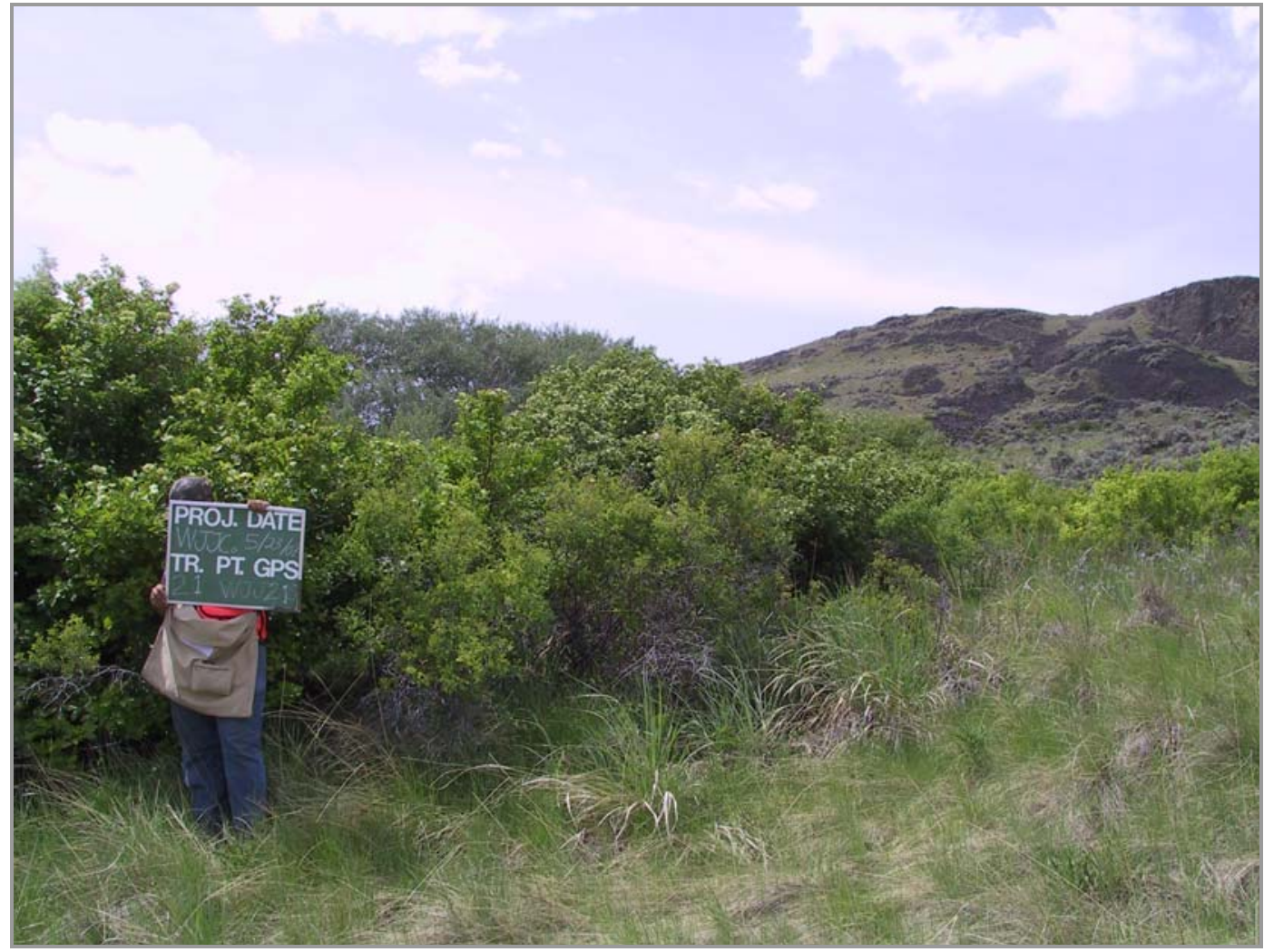

\section{Transect 22}

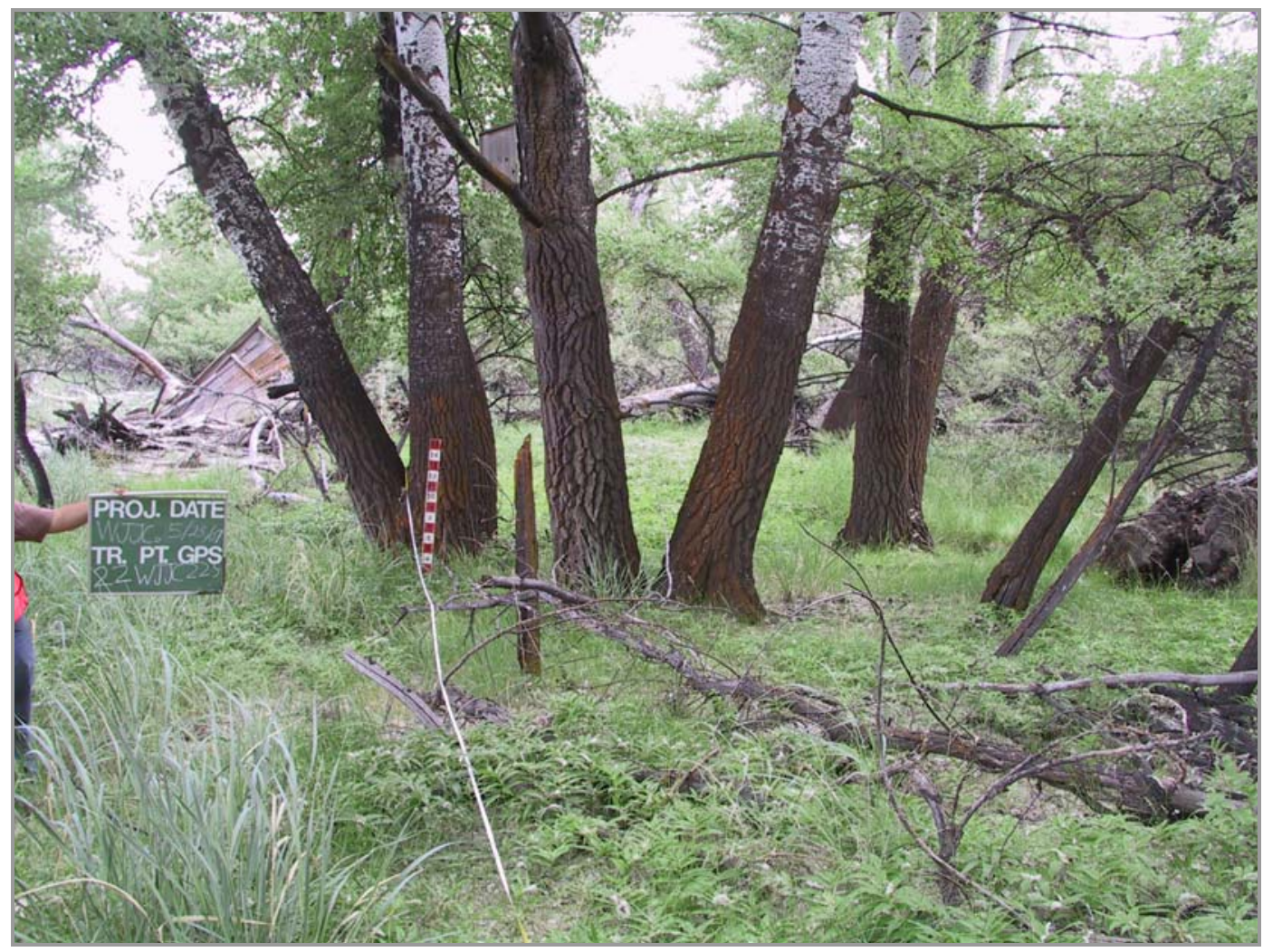


West Foster Creek Expansion Project

\section{Transect 23}

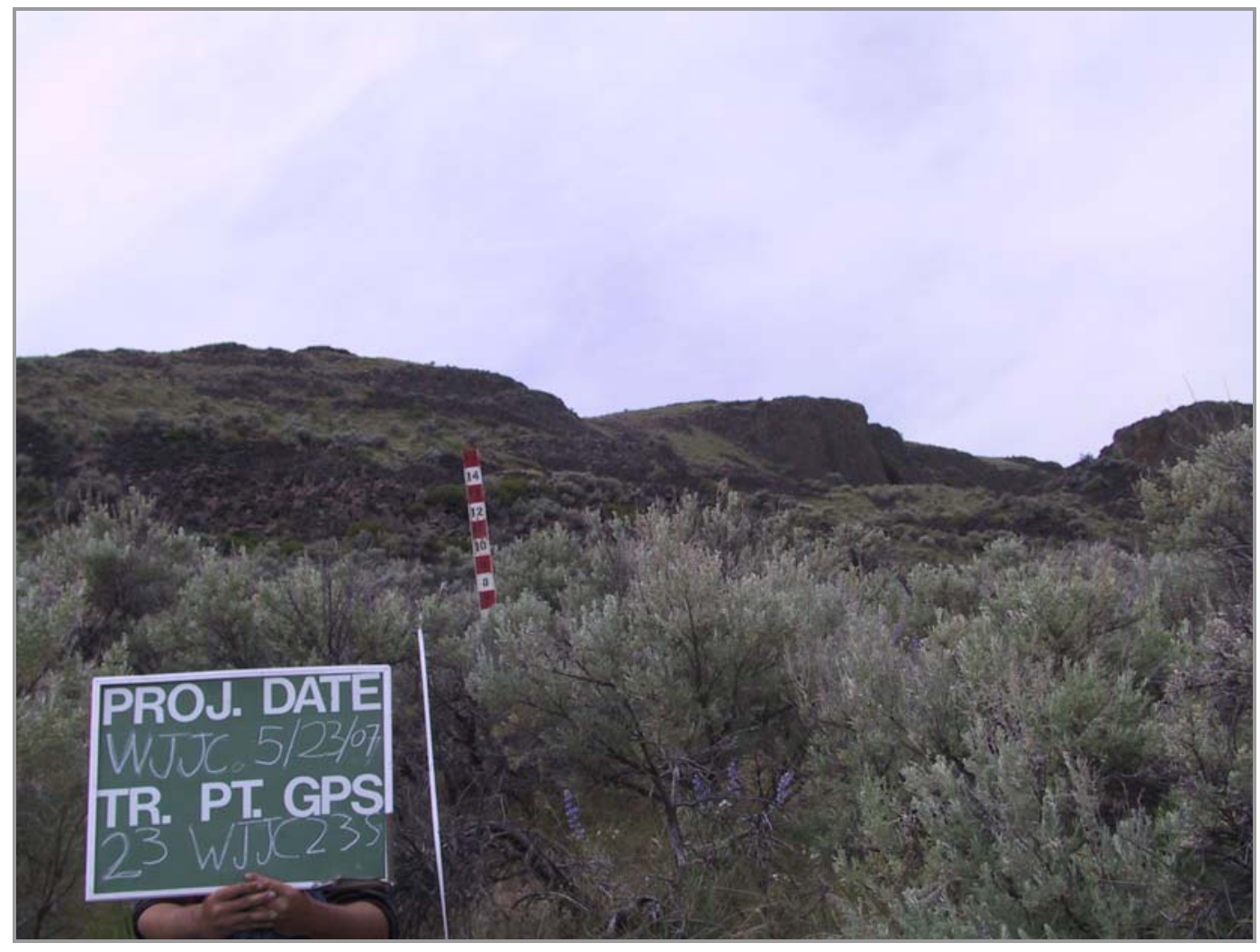

\section{Transect 24}

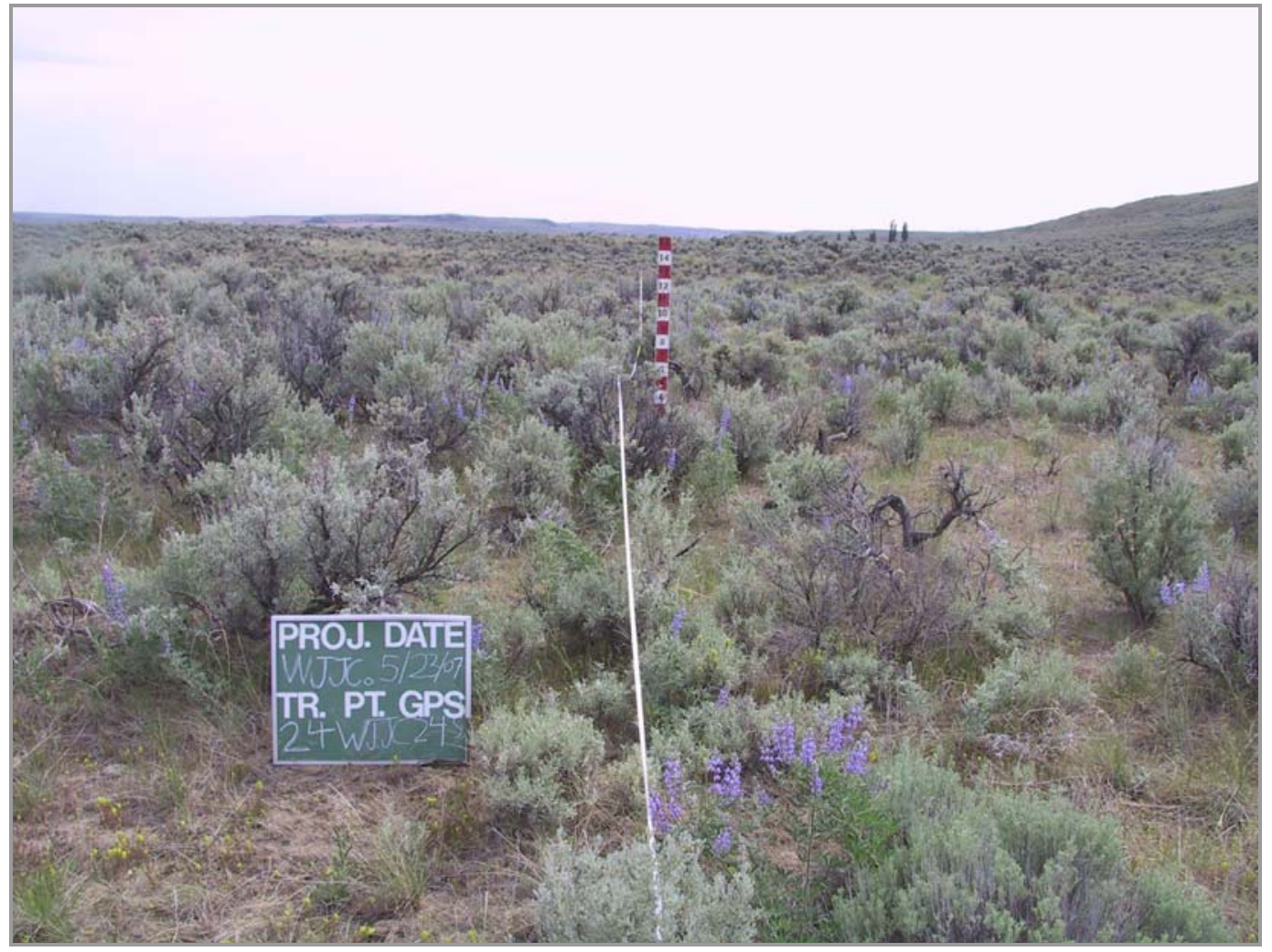


West Foster Creek Expansion Project

\section{Transect 25}

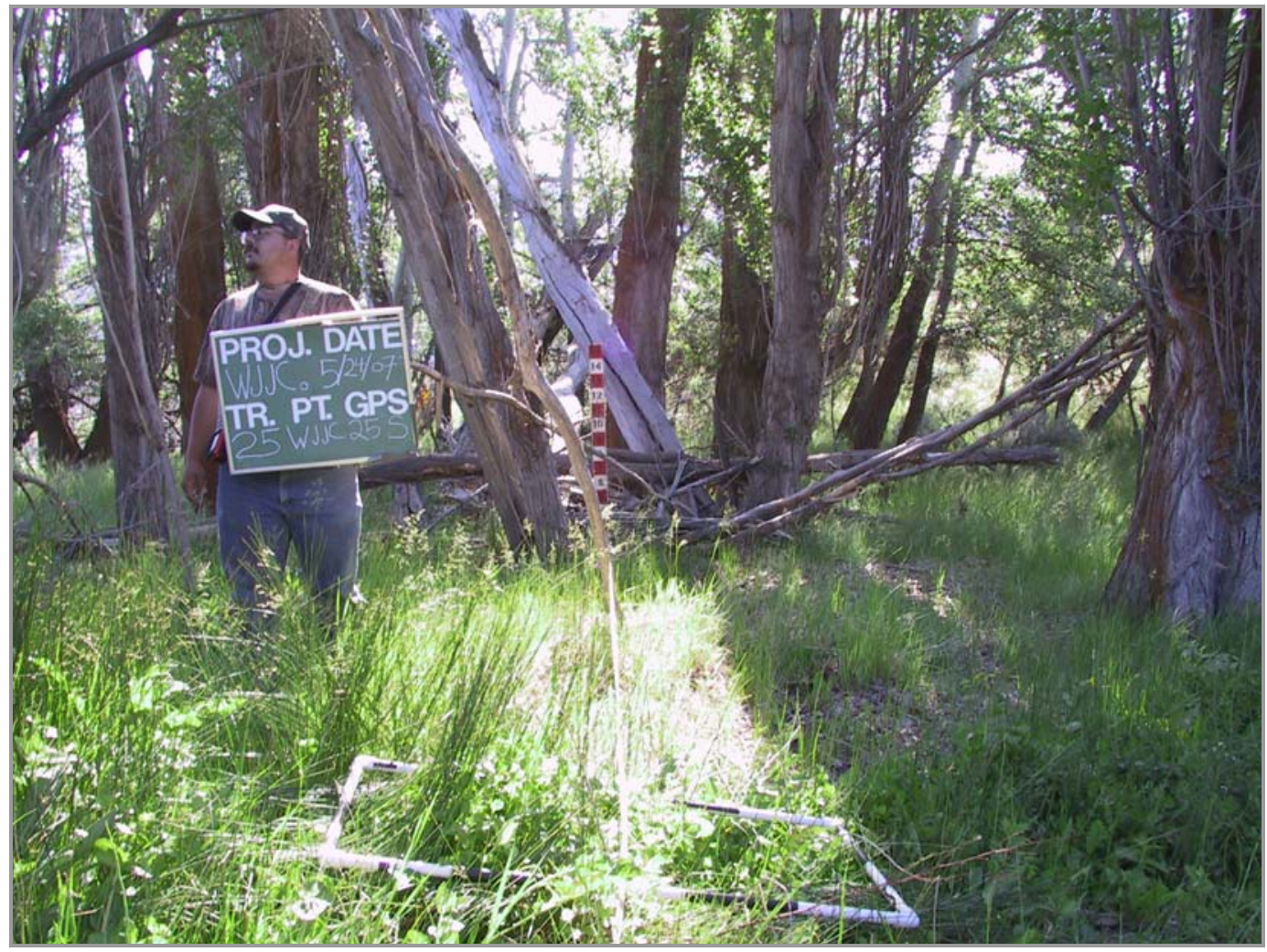

\section{Transect 26}

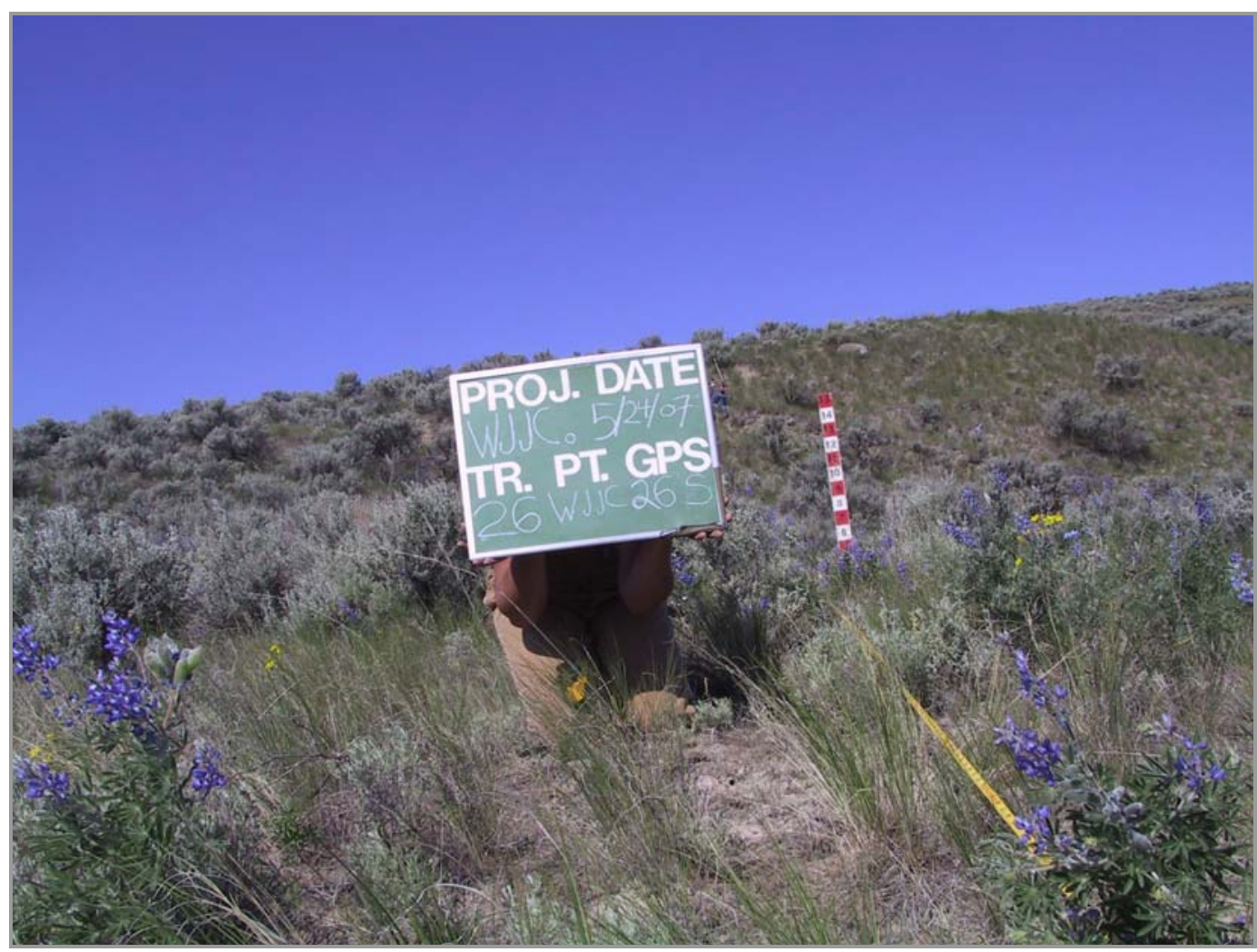


West Foster Creek Expansion Project

\section{Transect 27}

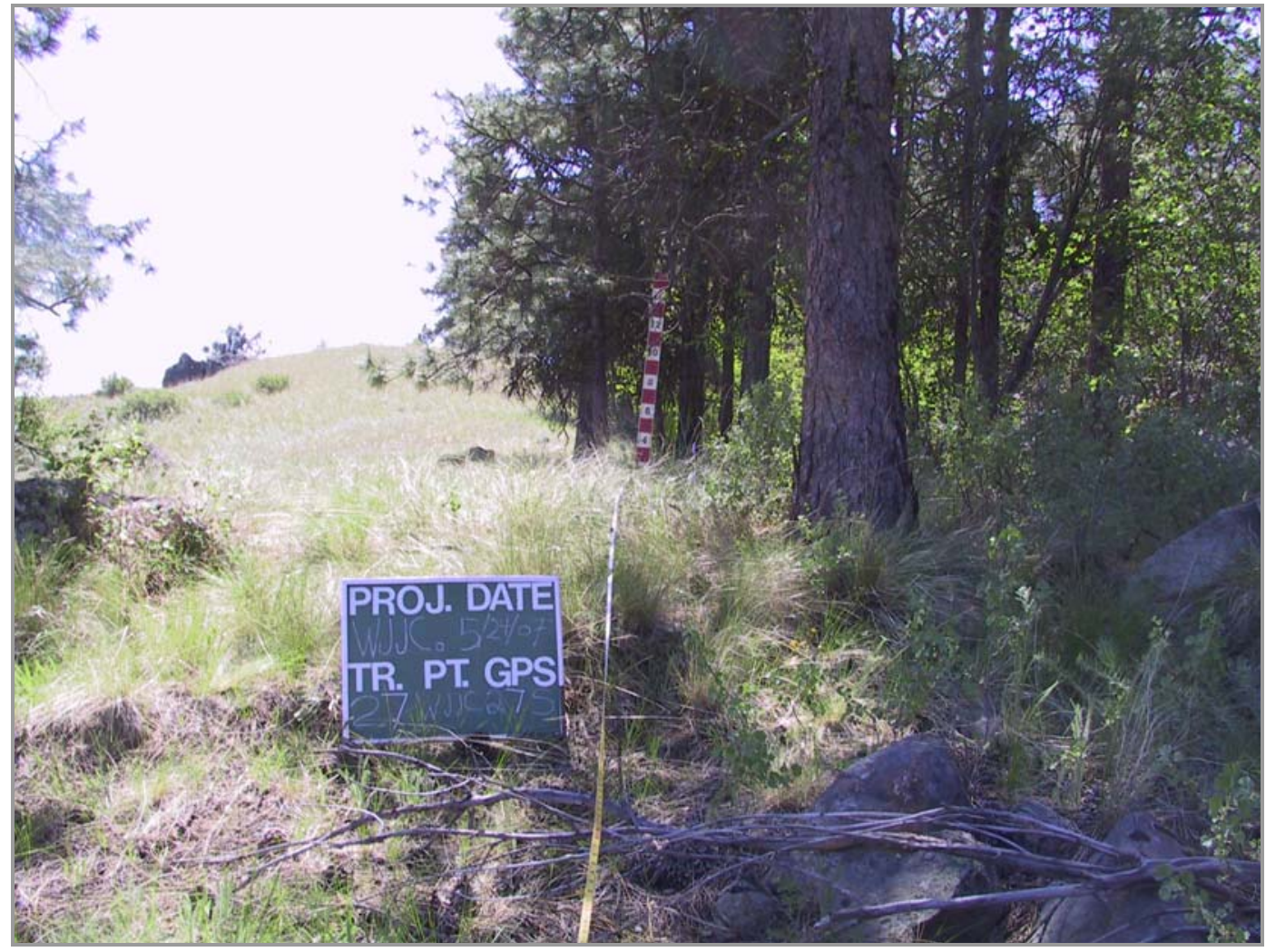

\section{Transect 28}

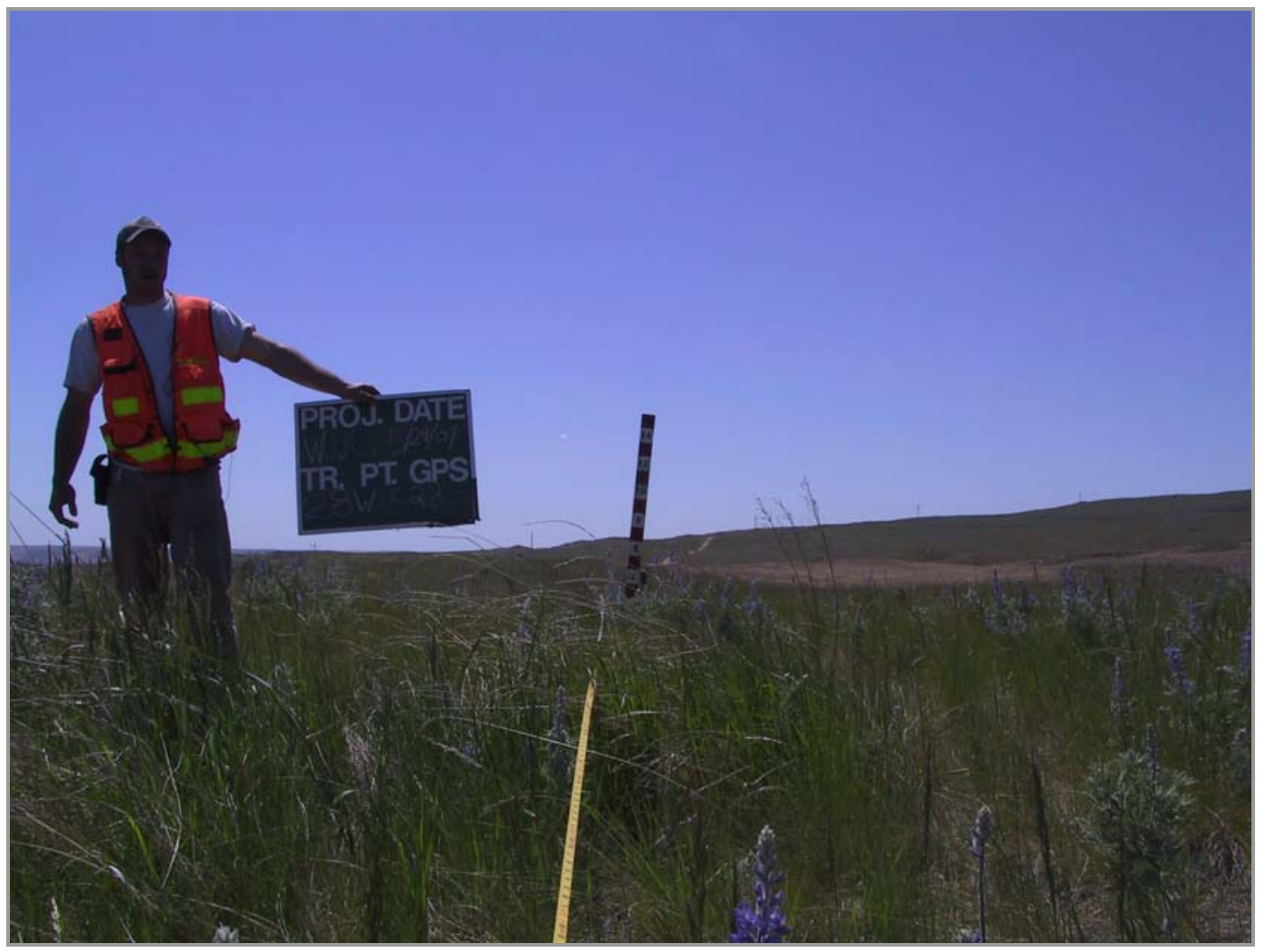


West Foster Creek Expansion Project

\section{Transect 29}

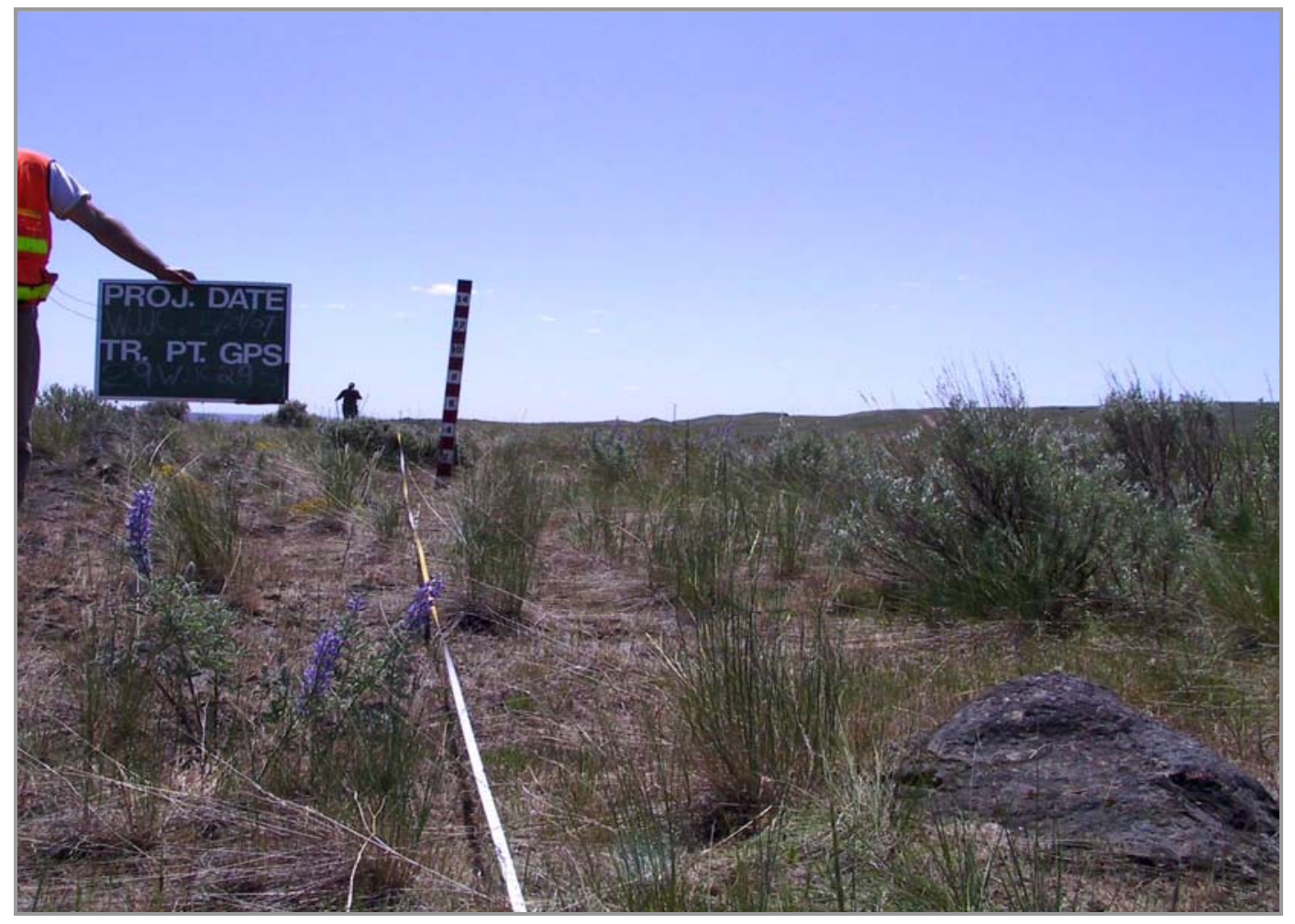

\section{Transect 30}

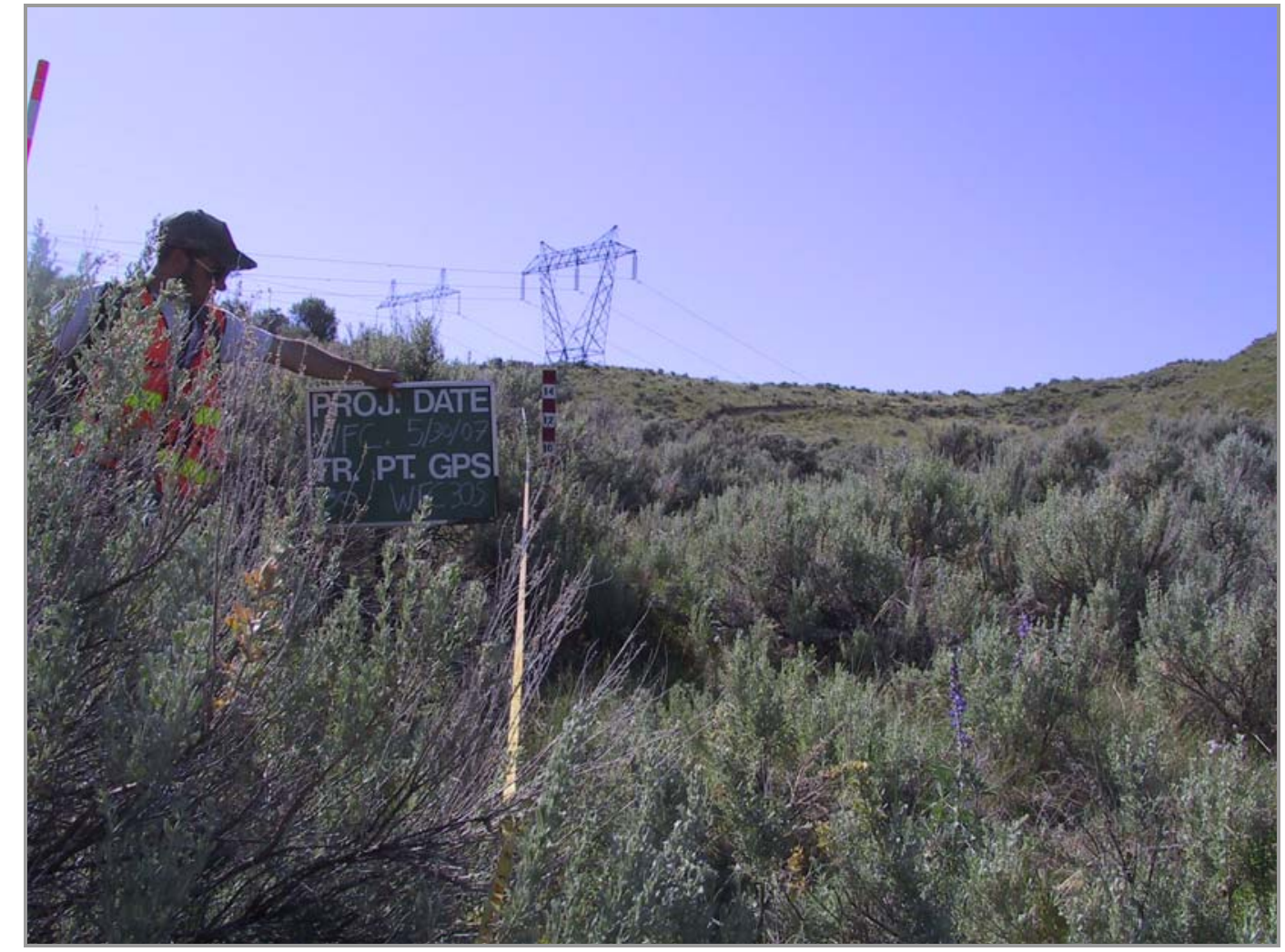


West Foster Creek Expansion Project

\section{Transect 31}

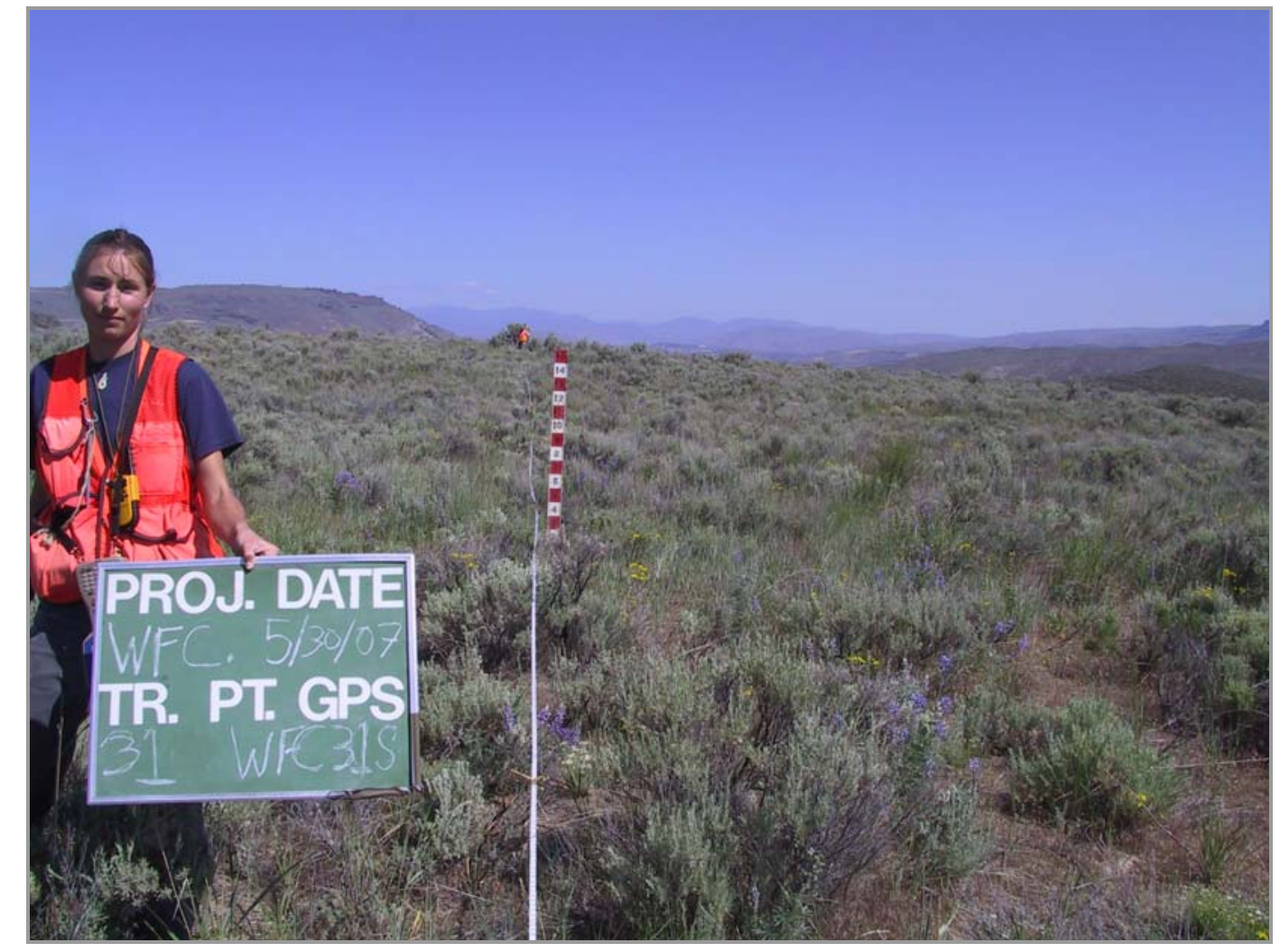

\section{Transect 32}

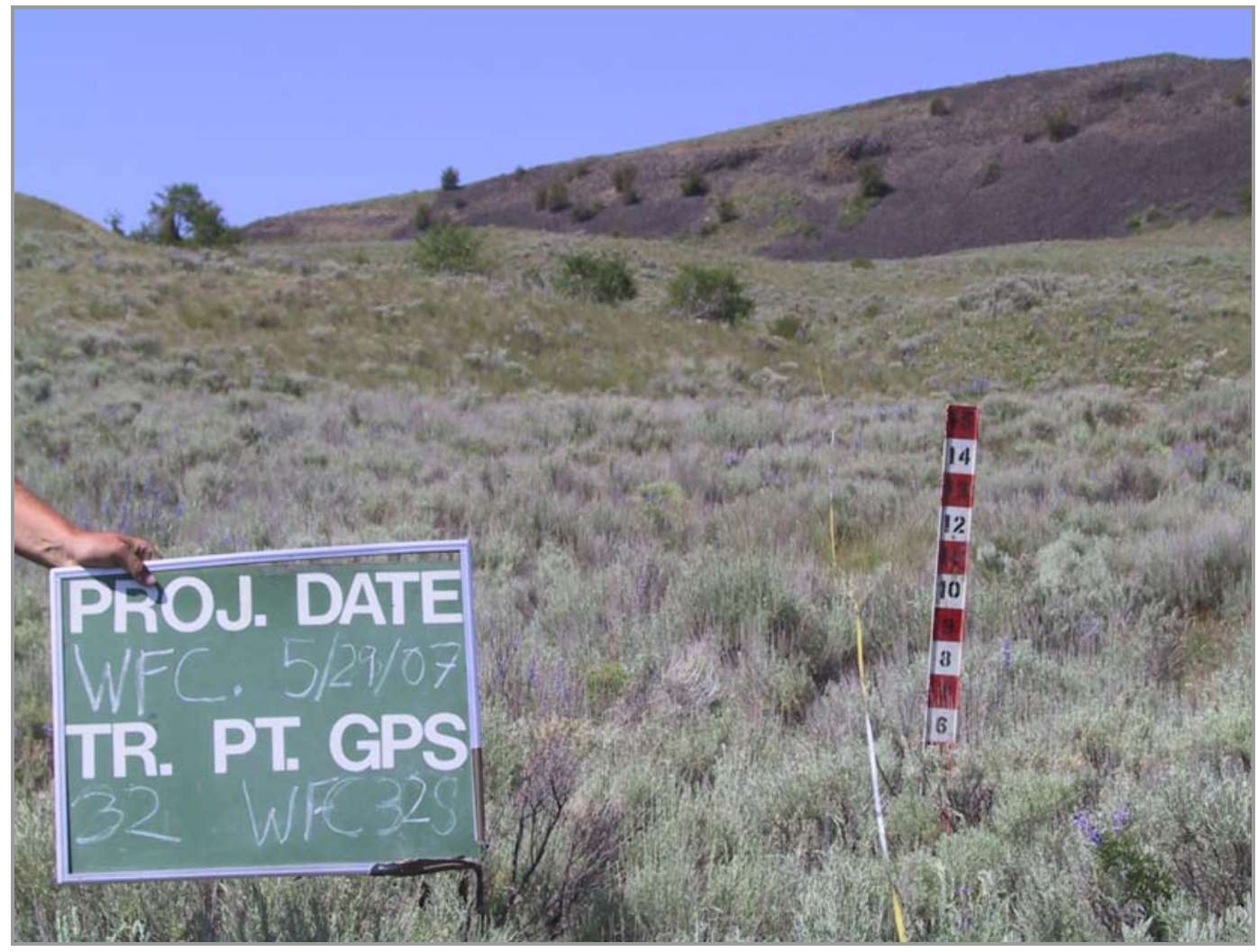


West Foster Creek Expansion Project

\section{Transect 33}

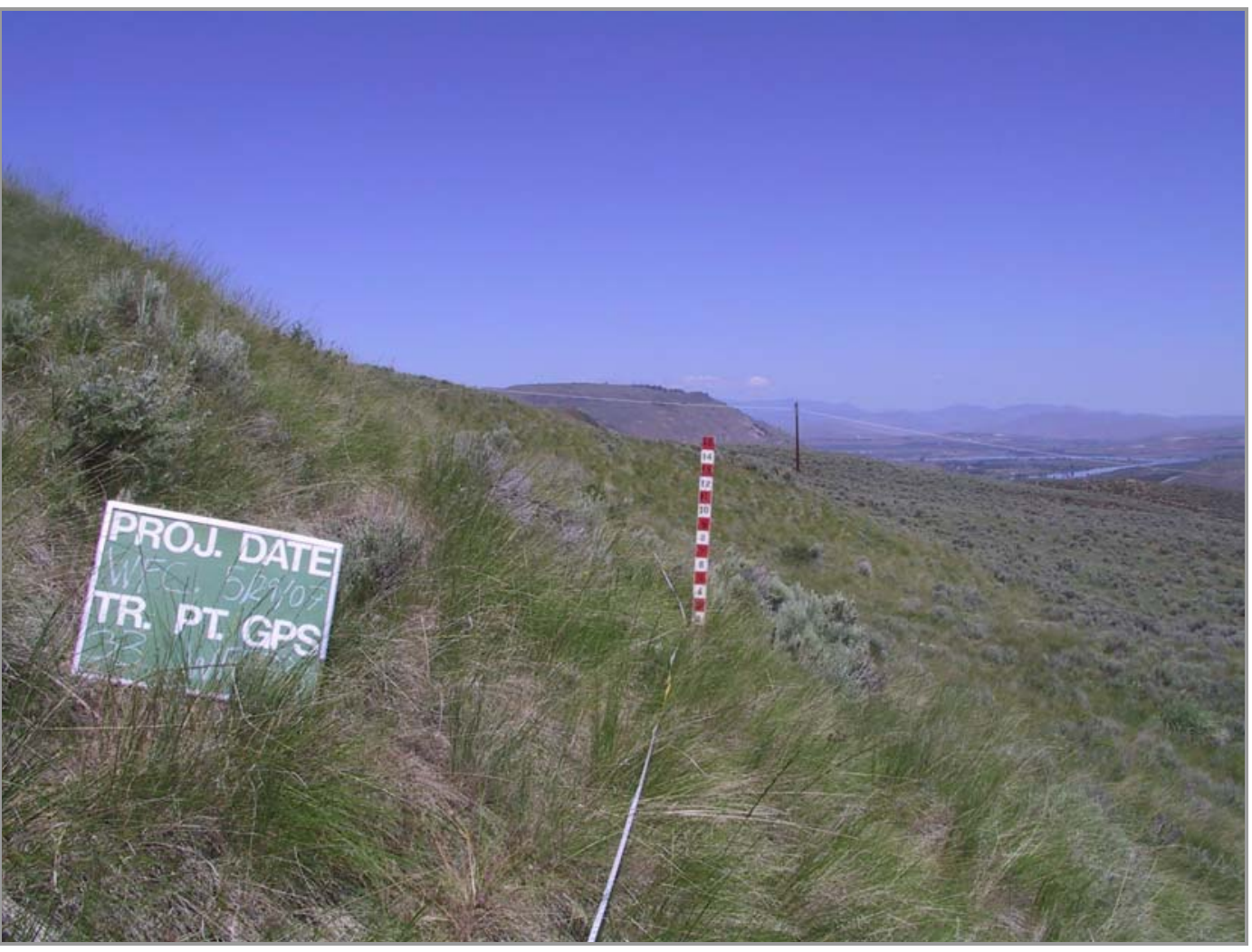

\section{Transect 34}

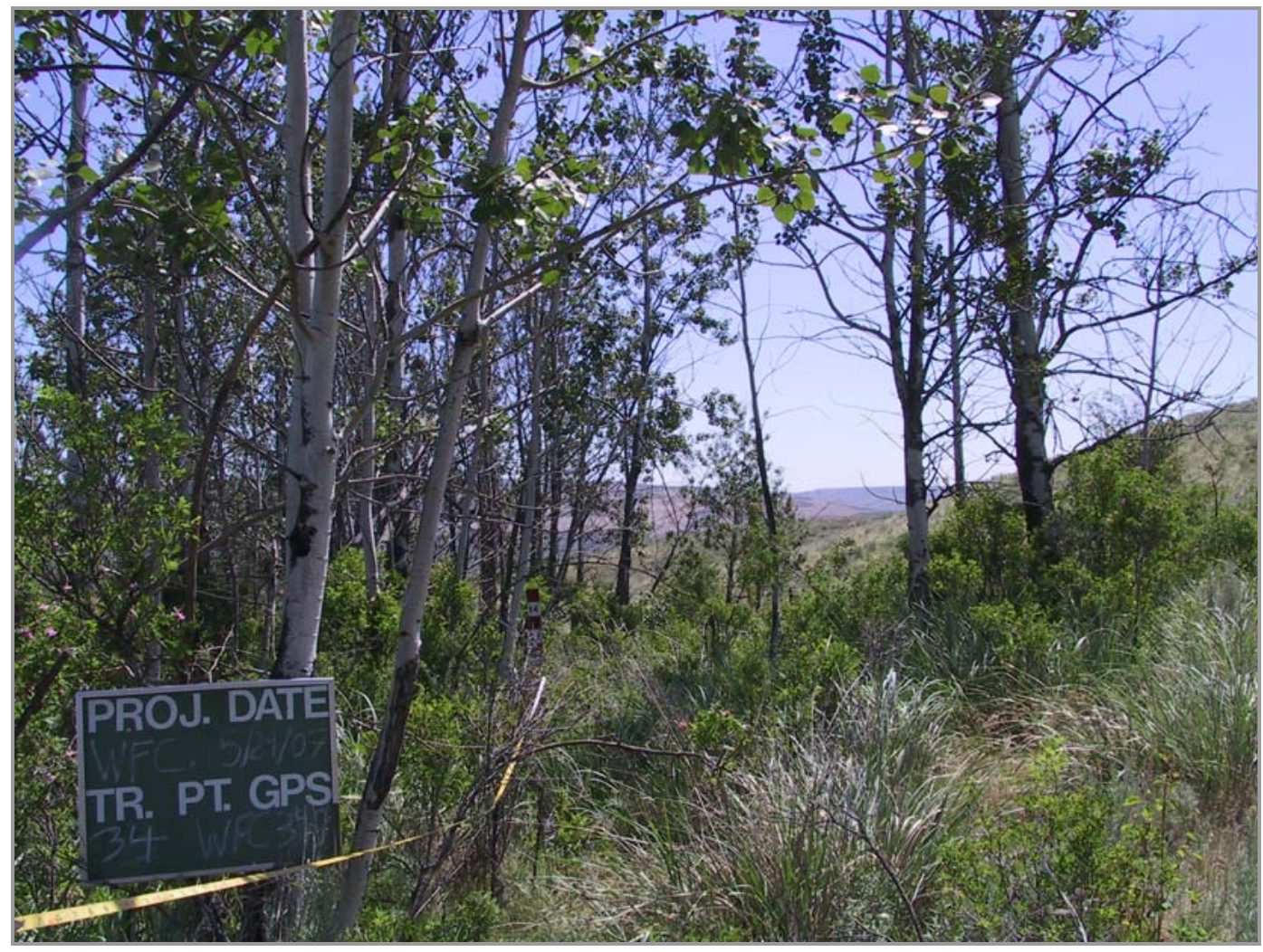


West Foster Creek Expansion Project

\section{Transect 35}

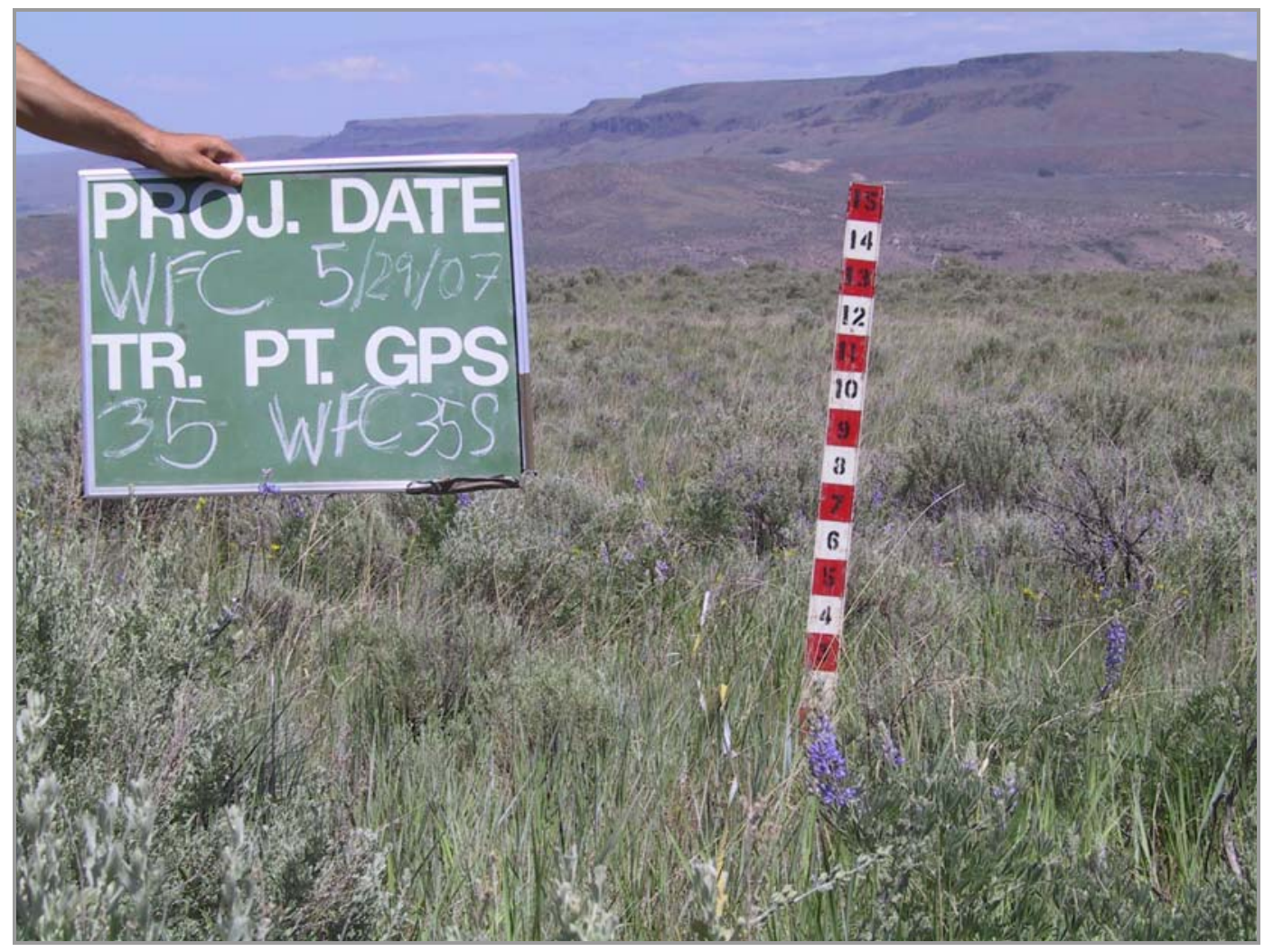

\section{Transect 36}

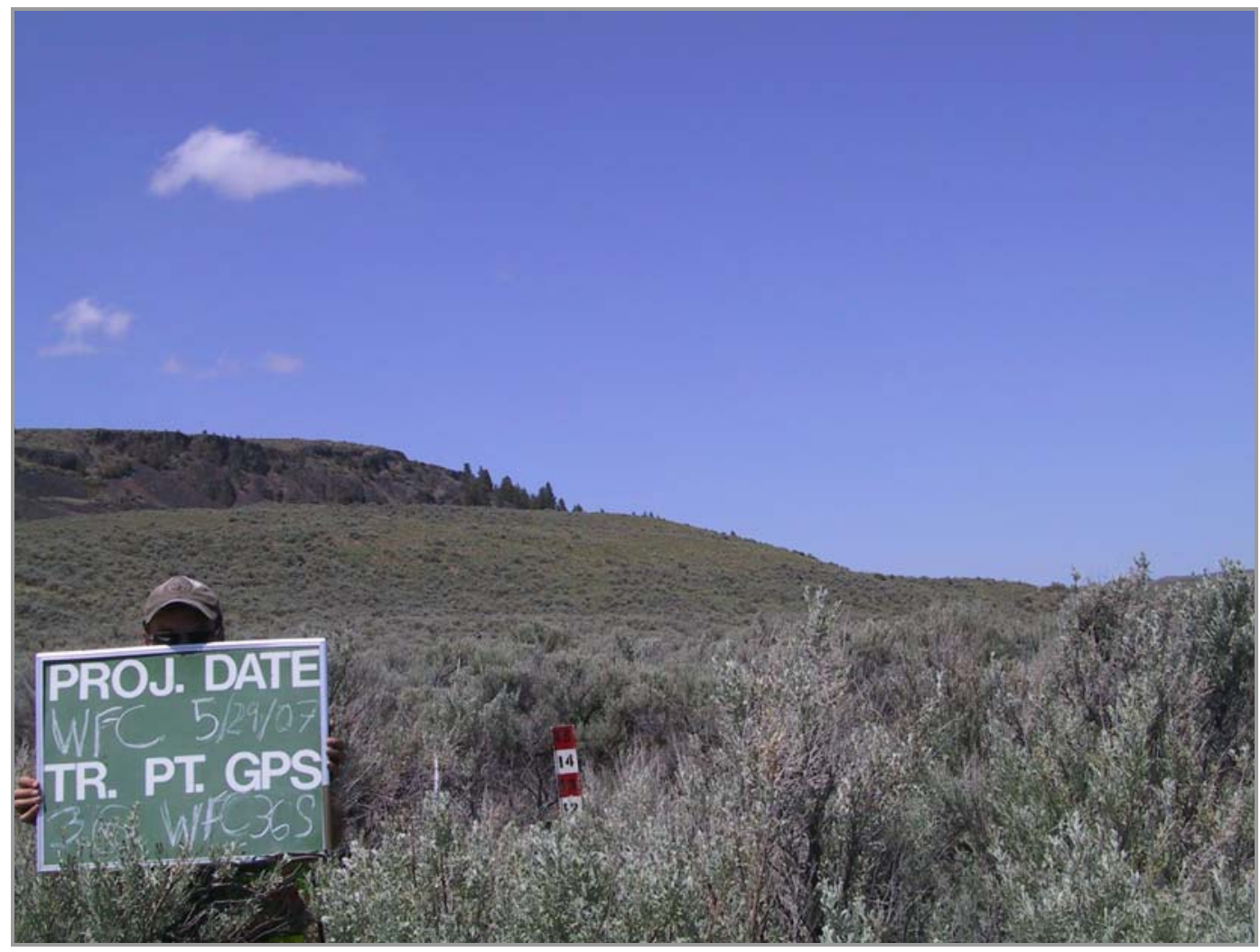


West Foster Creek Expansion Project

\section{Transect 37}

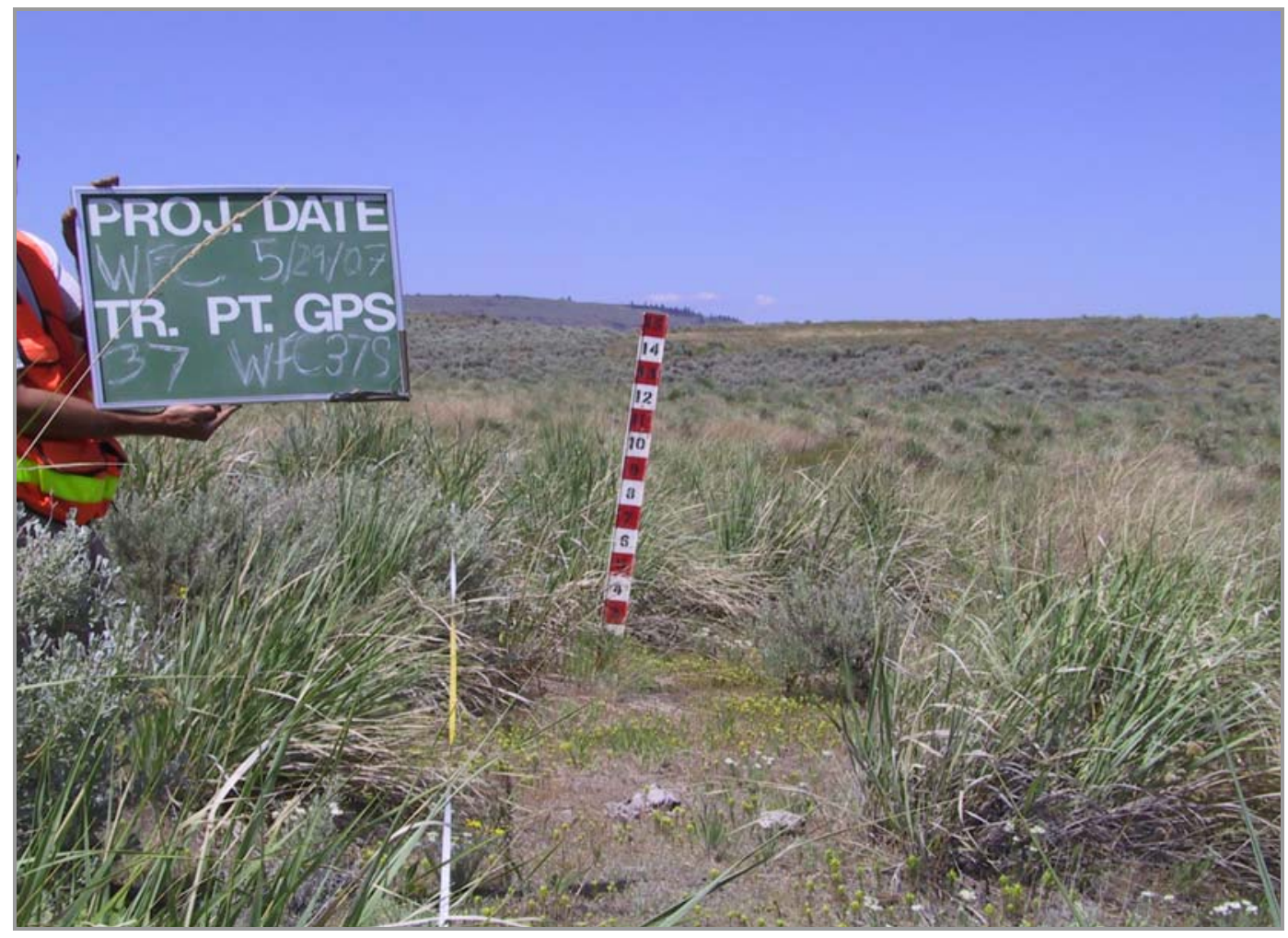

\section{Transect 38}

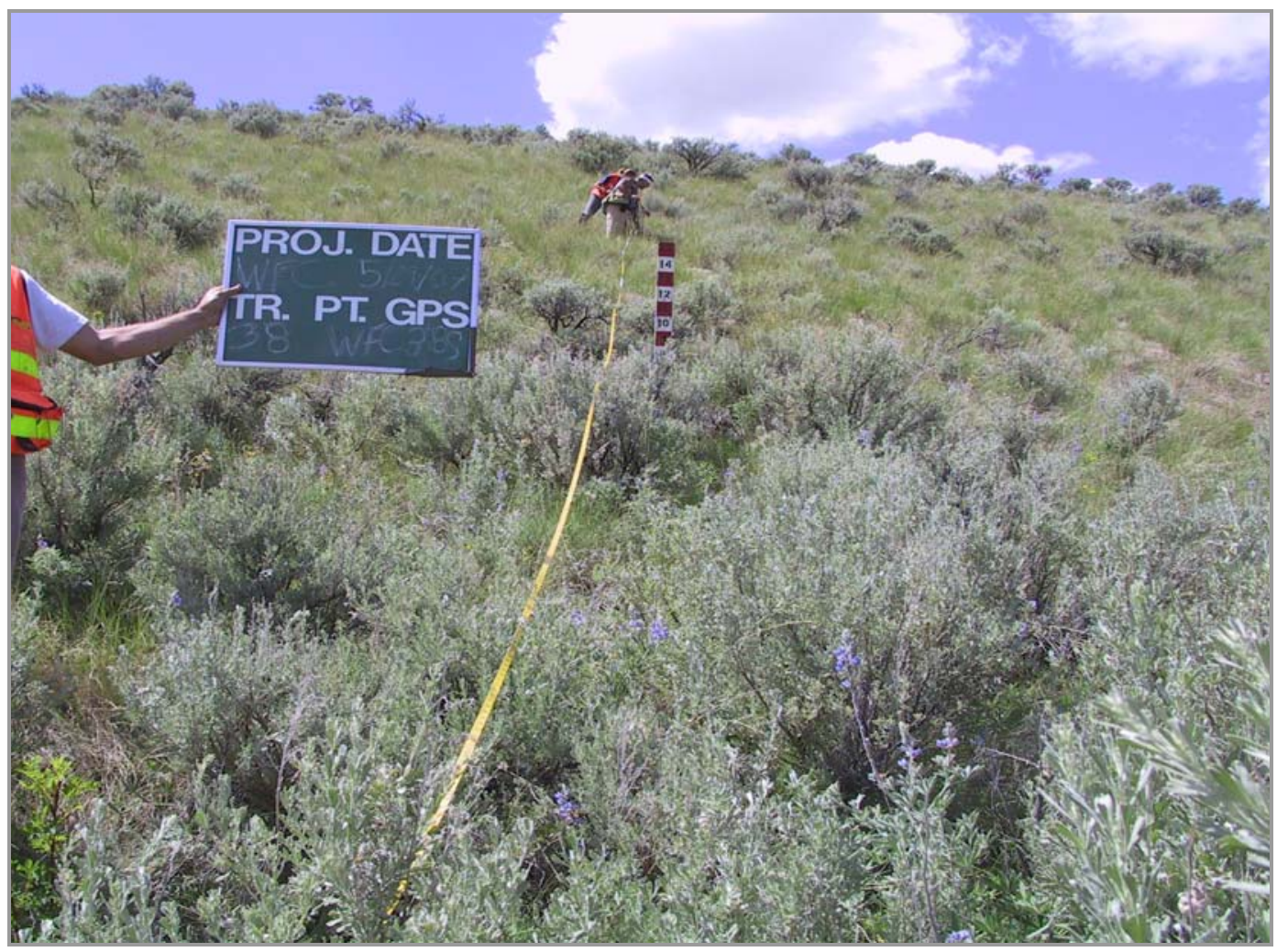


West Foster Creek Expansion Project

\section{Transect 39}

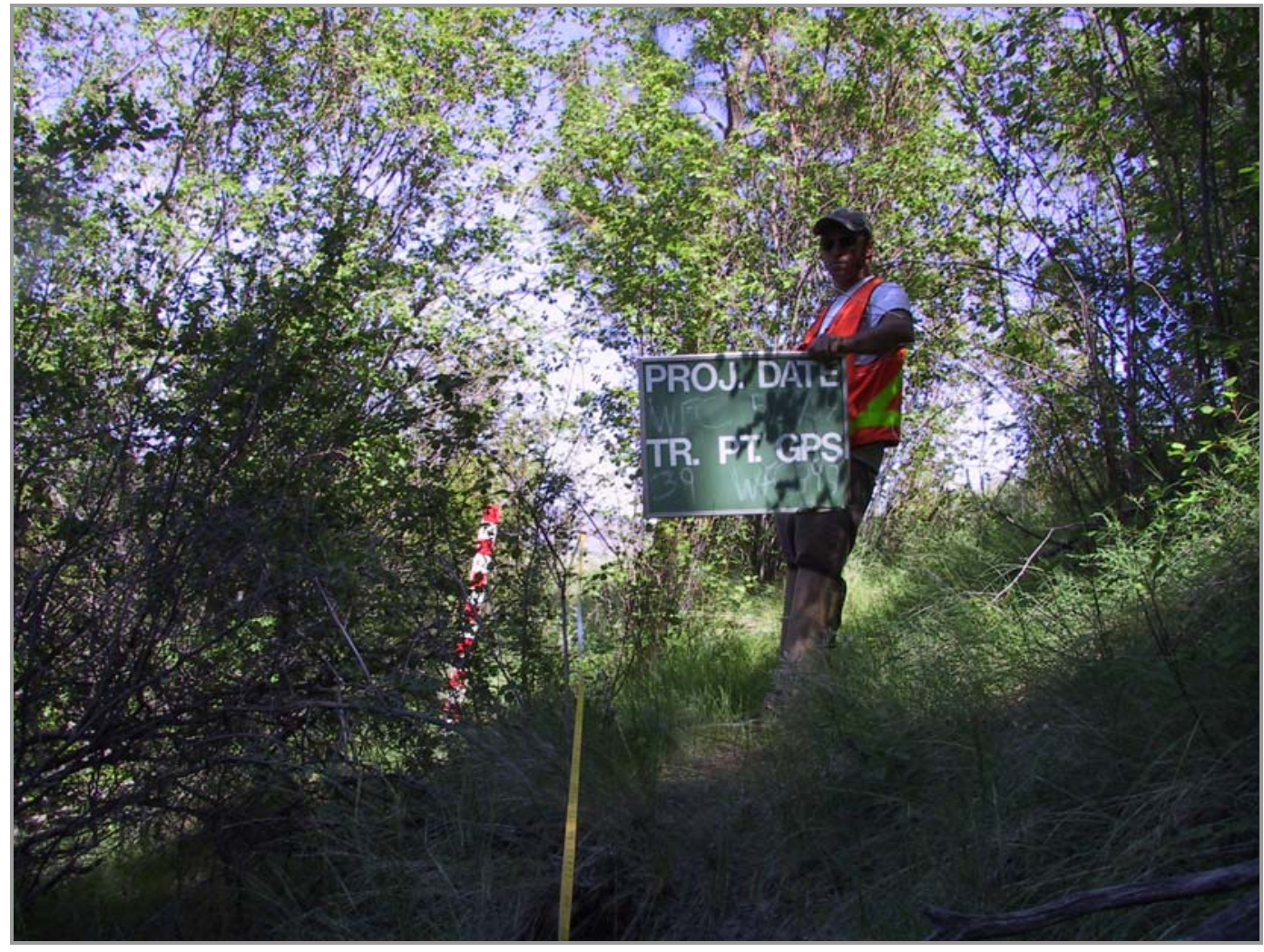

\section{Transect 40}

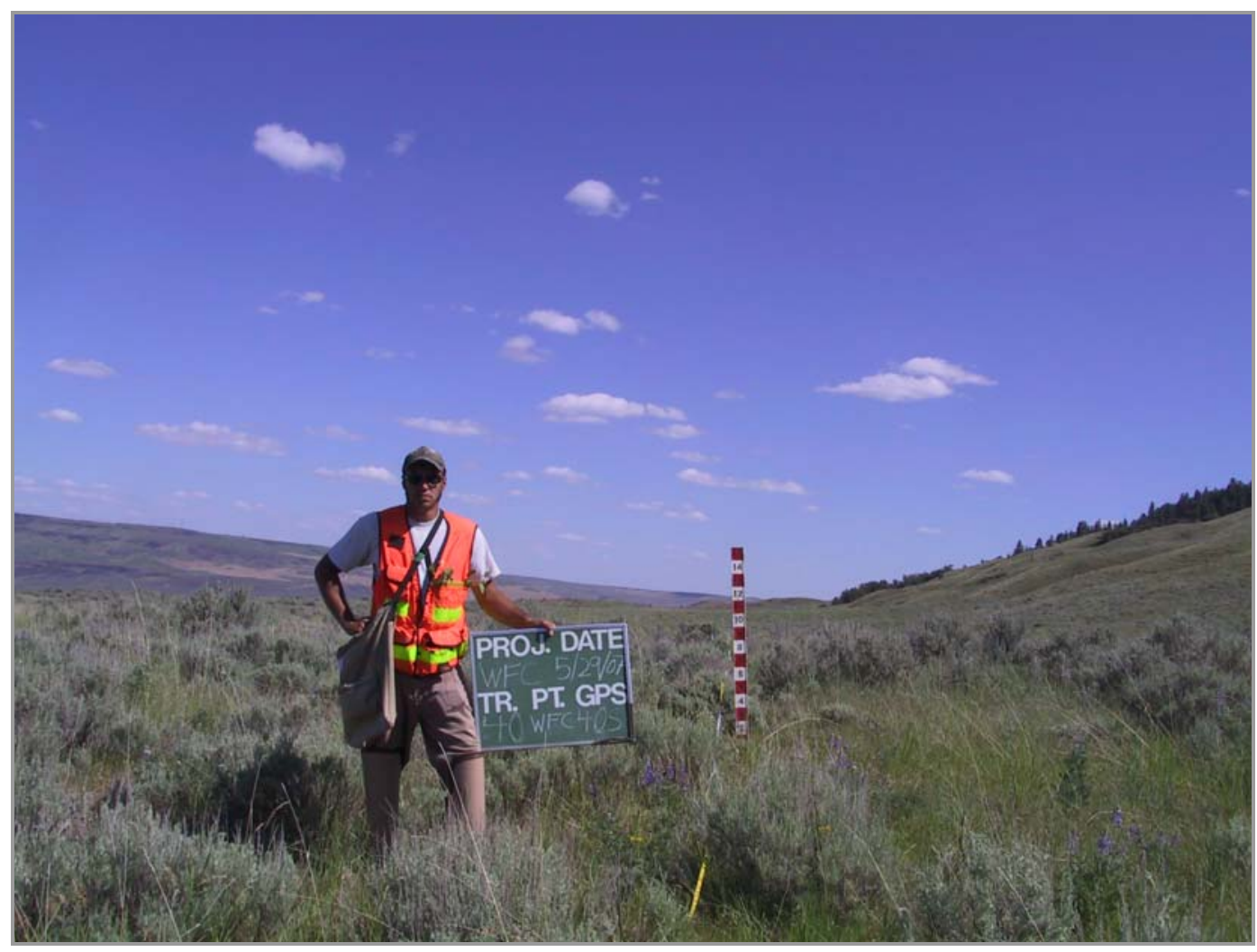


West Foster Creek Expansion Project

\section{Transect 41}

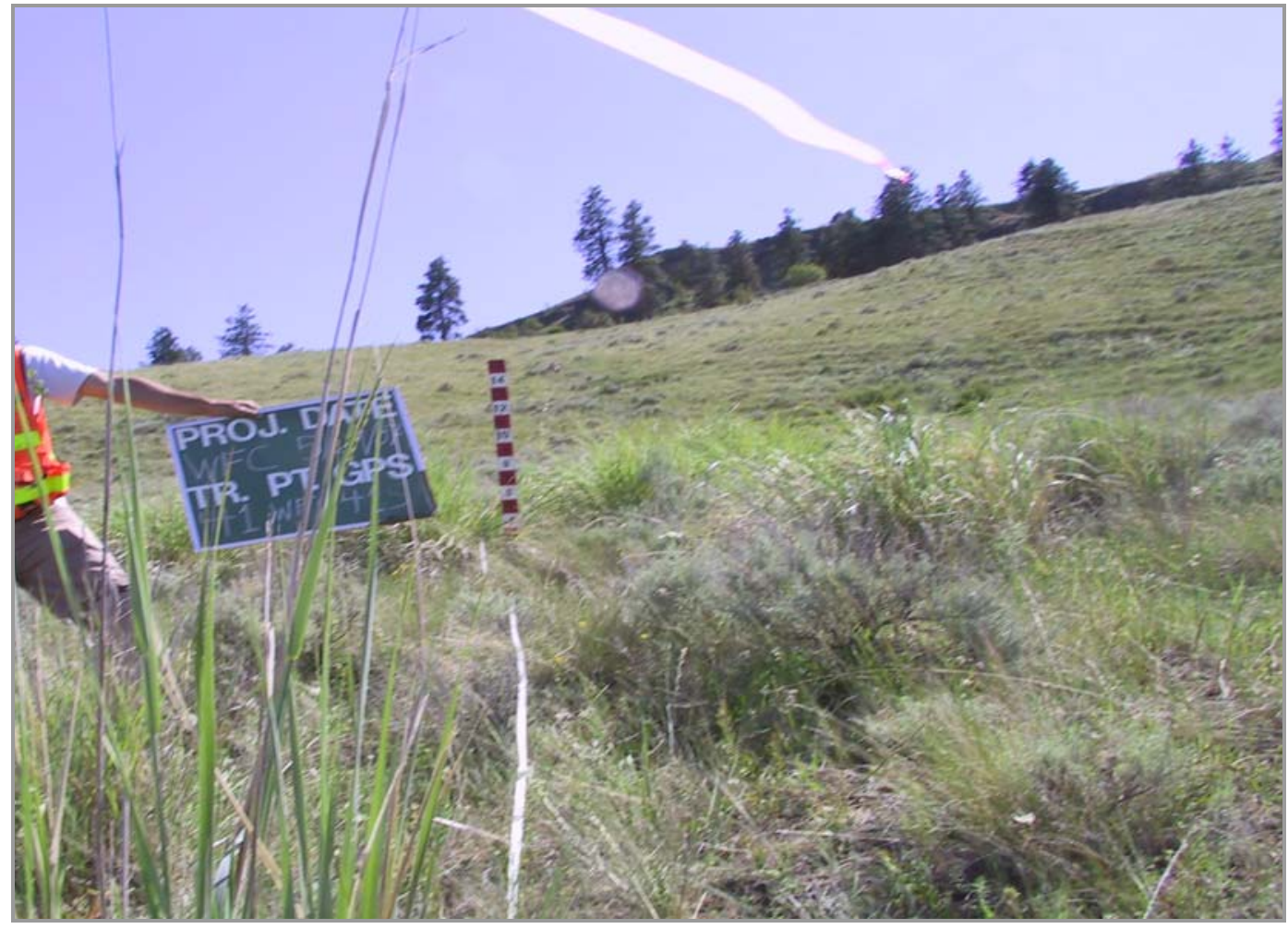

\section{Transect 42}

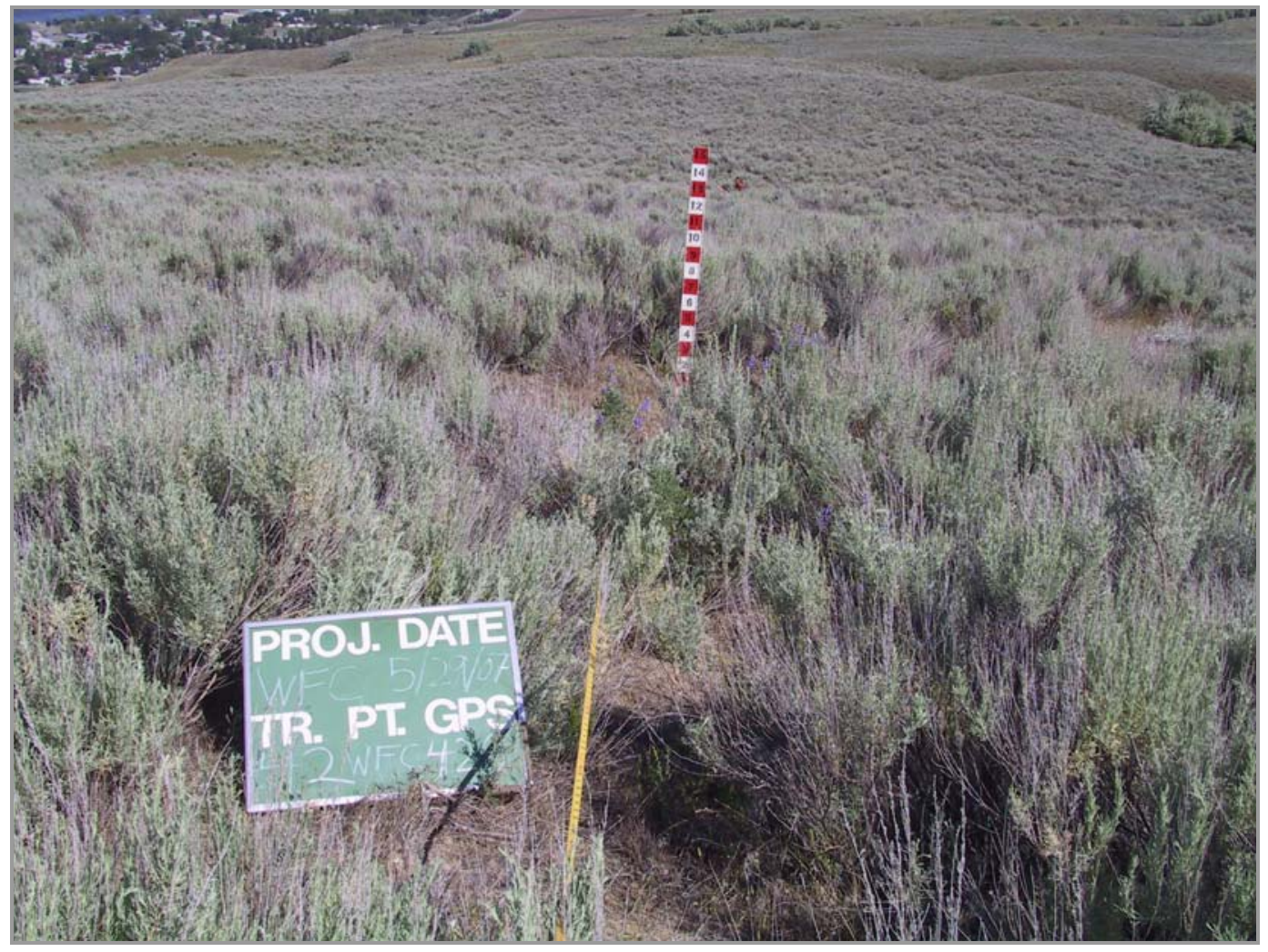


West Foster Creek Expansion Project

\section{Transect 50}

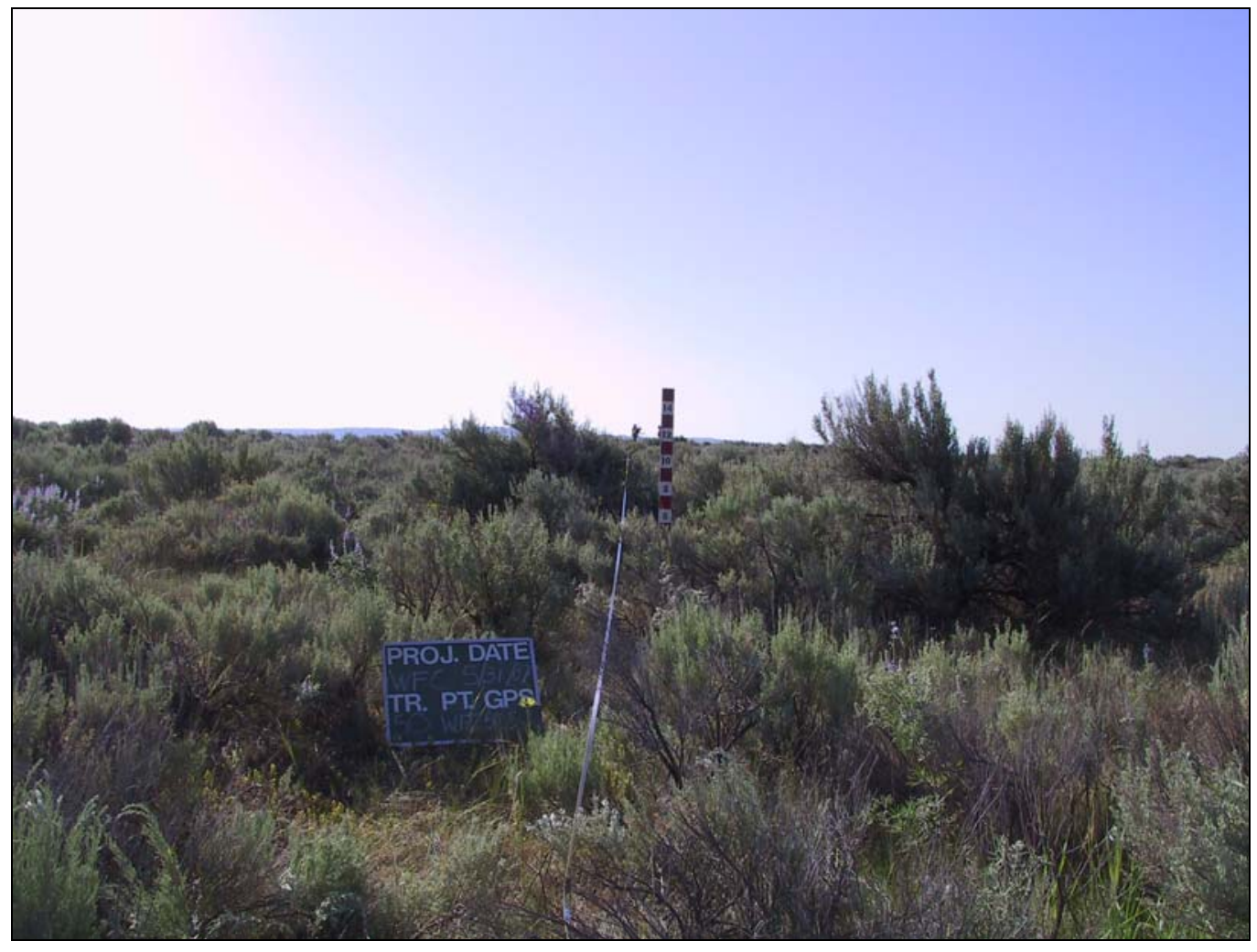

\section{Transect 51}

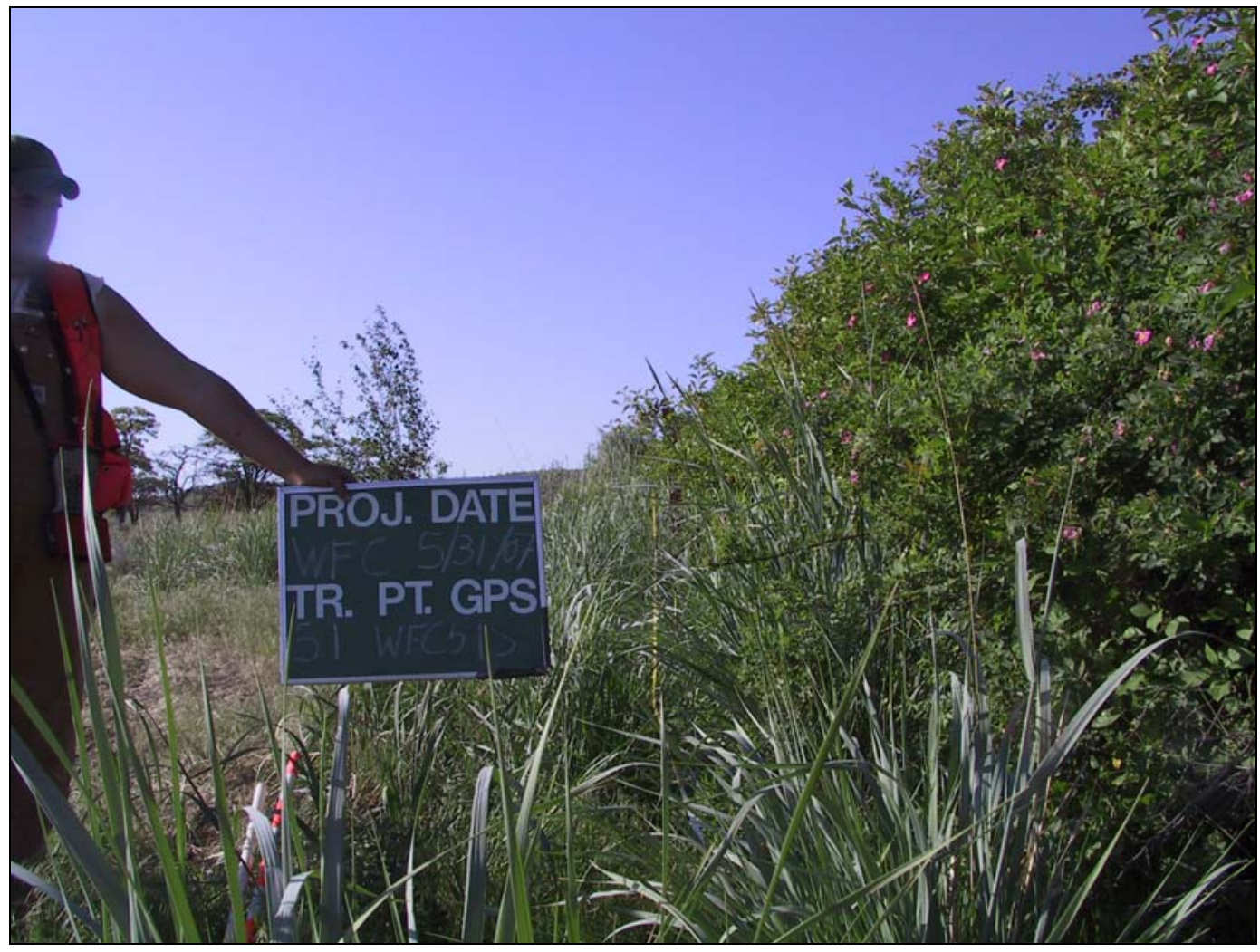


West Foster Creek Expansion Project

\section{Transect 52}

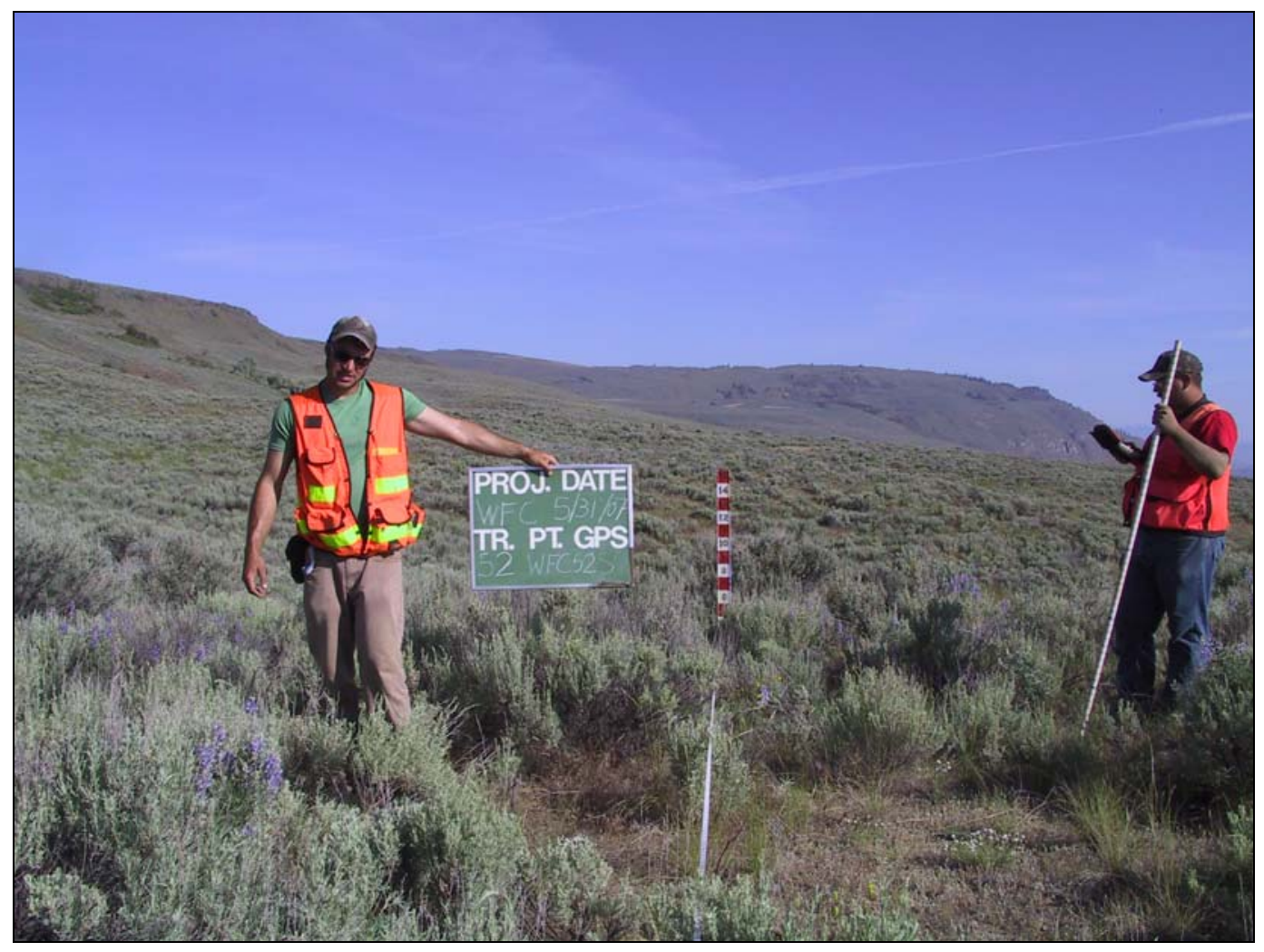

\section{Transect 53}

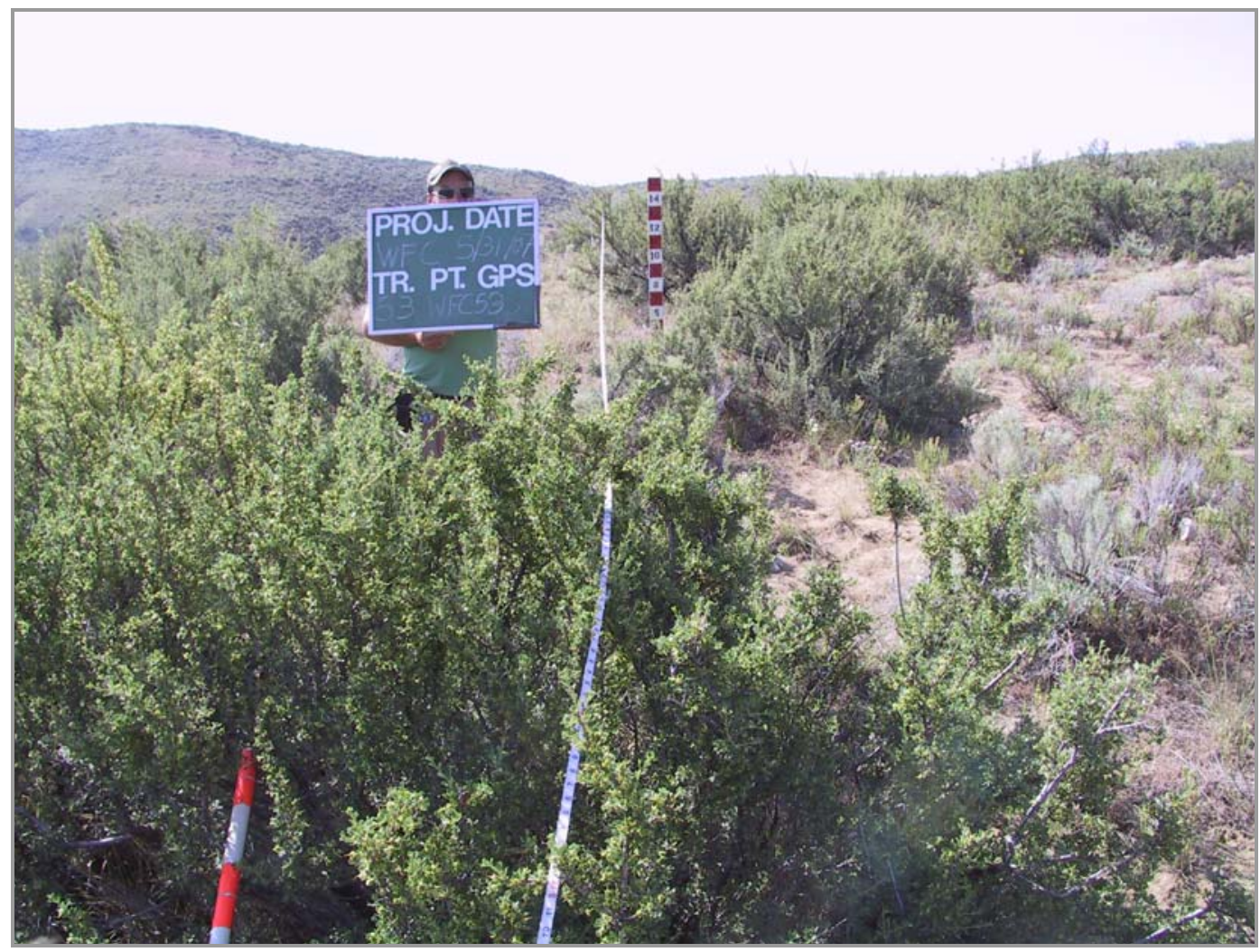


West Foster Creek Expansion Project

\section{Transect 54}

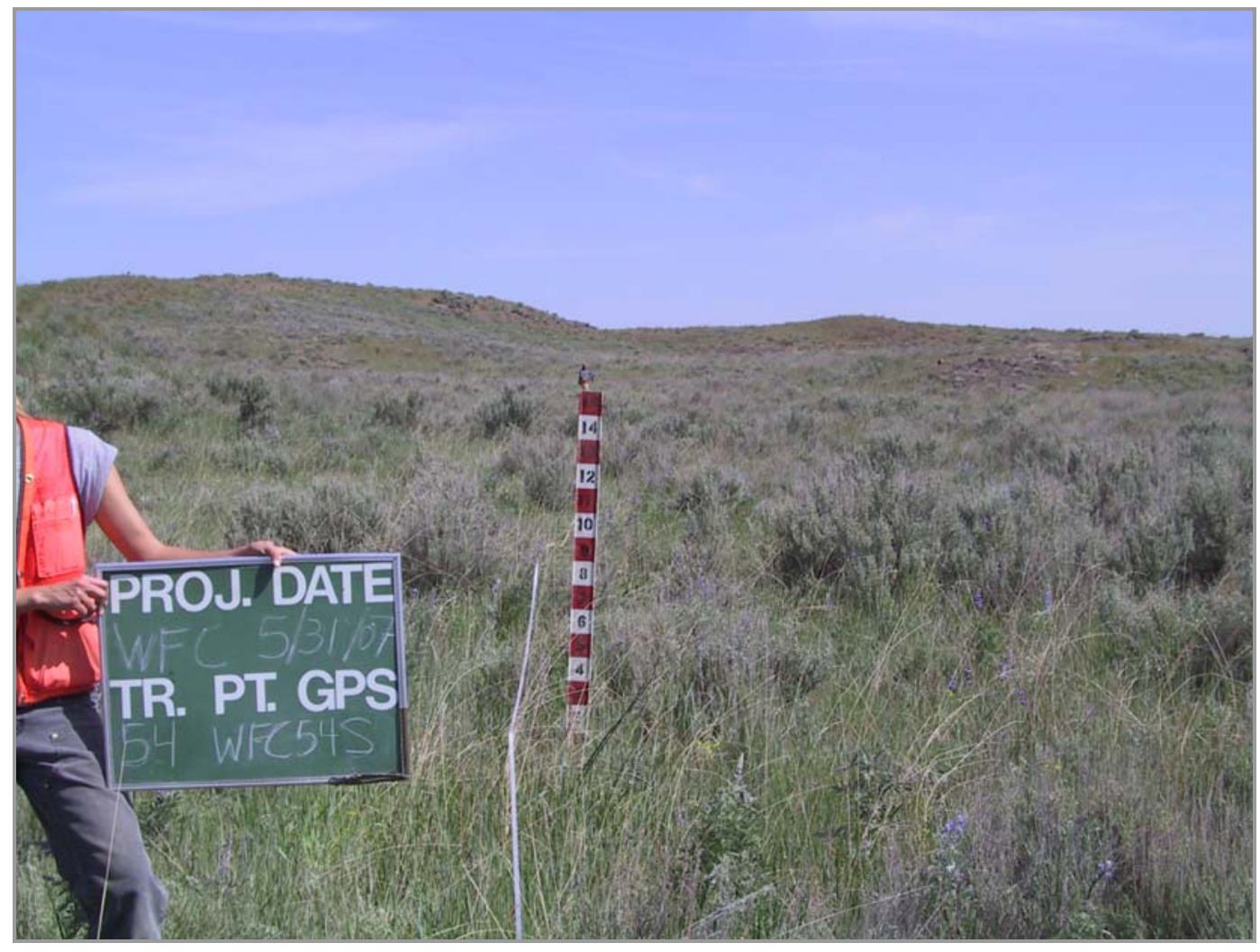

\section{Transect 55}

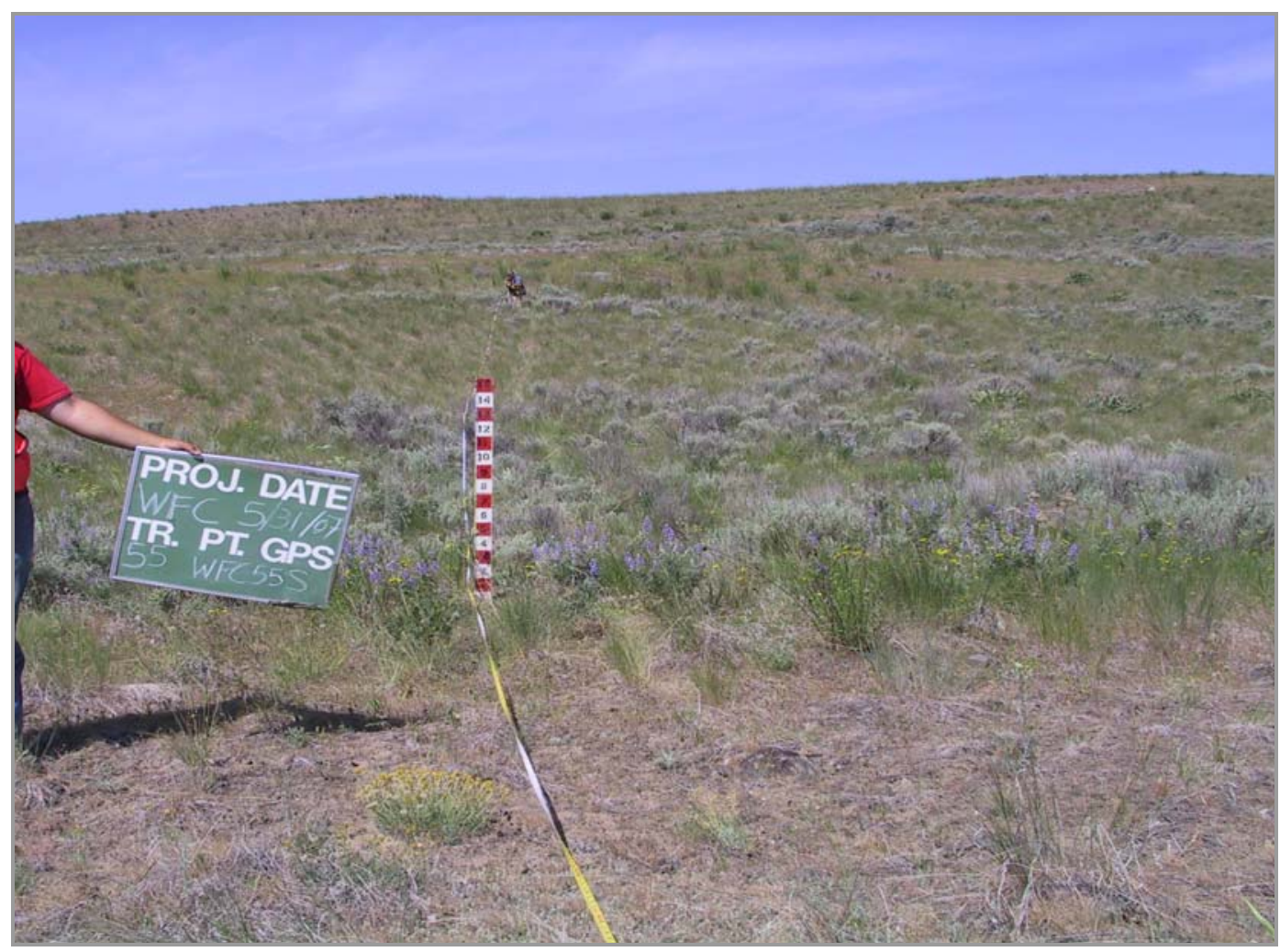

